\title{
MULTI-SIGNALS INVOLVED IN HUMAN JOINT HOMEOSTASIS
}

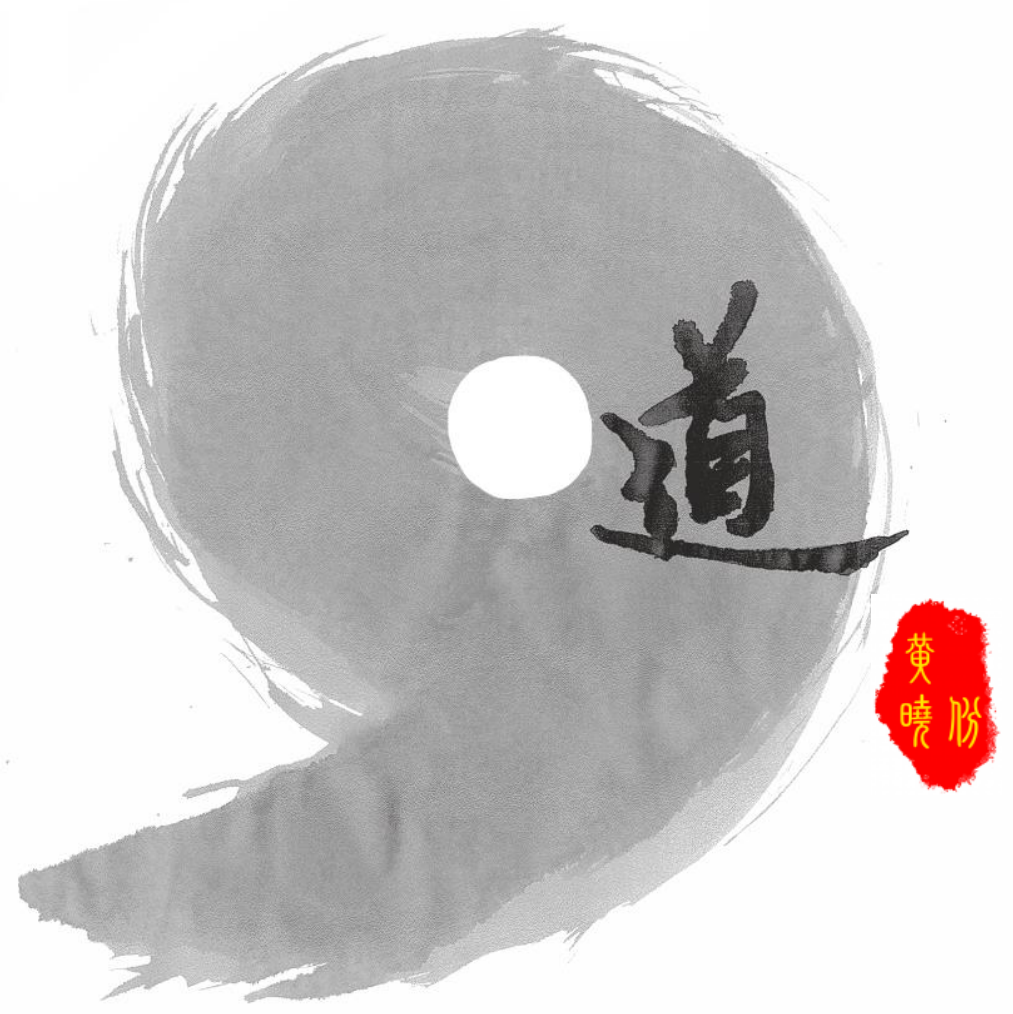

Xiaobin Huang 


\section{MULTI-SIGNALS INVOLVED IN HUMAN JOINT}

HOMEOSTASIS

Xiaobin Huang

2018 


\section{Graduation committee}

\section{Chairman:}

Prof. dr. J.W.M. Hilgenkamp

University of Twente

\section{Supervisor:}

Prof. dr. H.B.J. Karperien

University of Twente

\section{Members:}

Prof. dr. P. C. J. J. Passier

University of Twente

Prof. dr. M. M. A. E. Claessens

University of Twente

Dr. J. Prakash

University of Twente

Prof. dr. Peter van der Kraan

Radboud UMC Nijmegen

Dr. T. J. M. Welting

Maastricht University

\section{MULTI-SIGNALS INVOLVED IN HUMAN JOINT HOMEOSTASIS}

Xiaobin Huang

PhD Thesis, University of Twente, Enschede, The Netherlands

The research described in this thesis was performed in the department of Developmental Bioengineering (DBE), Faculty of Science and Technology (TNW), University of Twente, P.O. Box 217, 7500AE Enschede, The Netherlands.

ISBN: 978-90-365-4466-5

Cover design by Xiaobin Huang.

Copyright (C) 2018 by X. Huang. All rights reserved. 


\title{
MULTI-SIGNALS INVOLVED IN HUMAN JOINT HOMEOSTASIS
}

\author{
DISSERTATION
}

to obtain

the degree of doctor at the University of Twente,

on the authority of the rector magnificus,

Prof. dr. T.T.M. Palstra,

on account of the decision of the graduation committee,

to be publicly defended

on Friday, $19^{\text {th }}$ of January, 2018 at $12.45 \mathrm{hrs}$.

by

\section{Xiaobin Huang}

Born on $1^{\text {st }}$ of September, 1980

In Chongqing, China 
This dissertation has been approved by

Supervisor: Prof. dr. H.B.J. Karperien (University of Twente) 


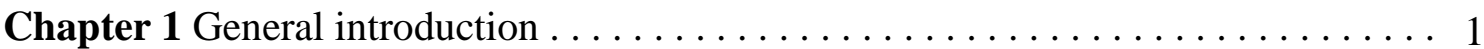

Chapter 2 The regulatory role of signaling crosstalk in hypertrophy of MSCs and human articular chondrocytes

Chapter 3 The expression of DKK1 and FRZB are negatively correlated to local inflammation and OA severity . . .

Chapter 4 Effects of small molecules BIO and PKF118-310 on chondrogenic differentiation of human mesenchymal stem cells 70

Chapter 5 Hypoxia greatly promotes the redifferentiation of human chondrocytes and diminishes the difference between health and osteoarthritic chondrocytes

Chapter 6 Co-treatment of TGF- $\beta 3$ and BMP-7 is superior in stimulating chondrocyte redifferentiation in both hypoxia and normoxia compared to single treatments

Chapter 7 Bihead VHHs efficiently direct BMP7 to bone in tissue engineering

Chapter 8 General discussion and summary

Summary

Acknowledgements

Curriculum Vitae

List of Publications . 


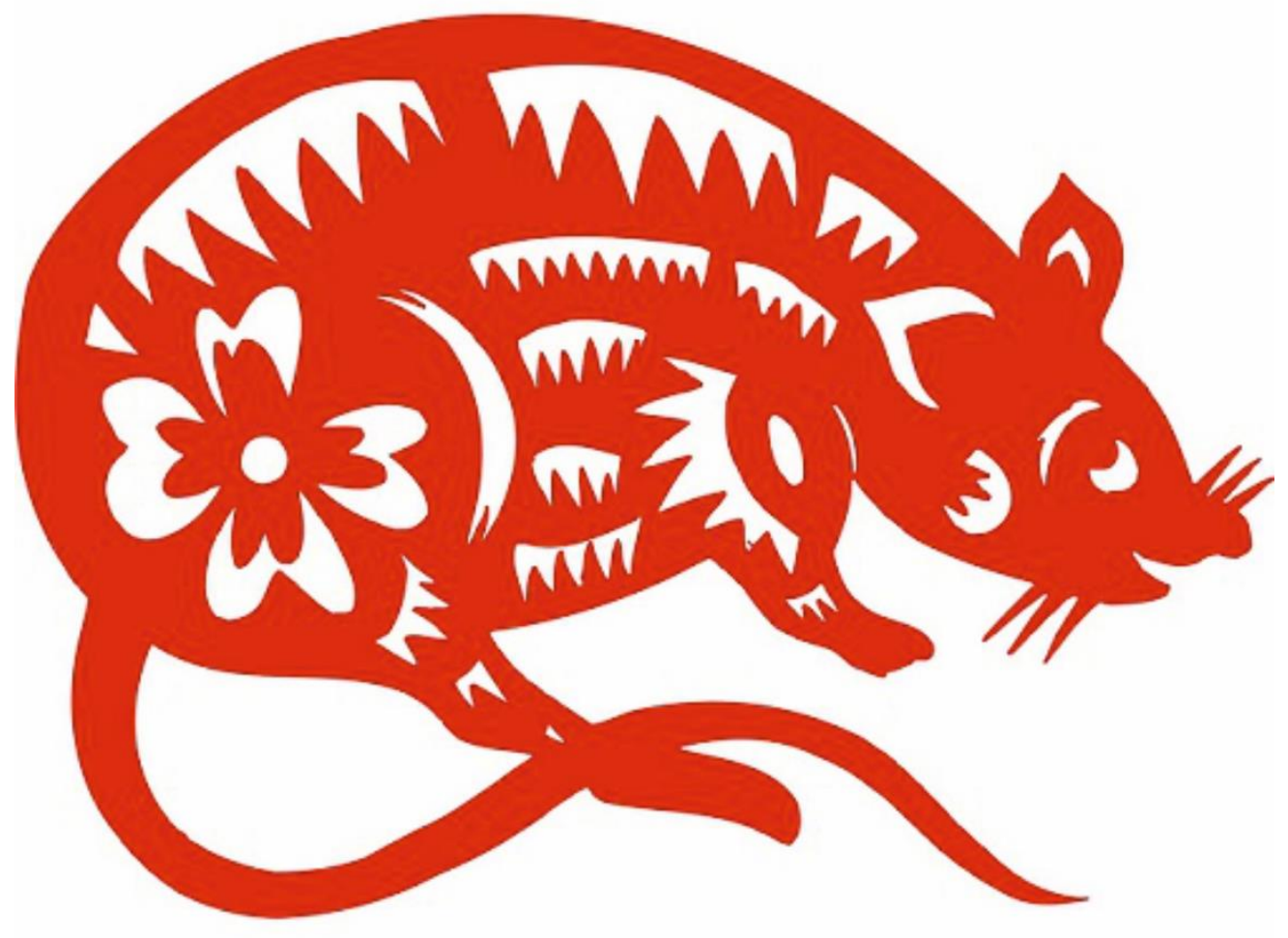




\section{Chapter 1}

\section{General Introduction and thesis outline}

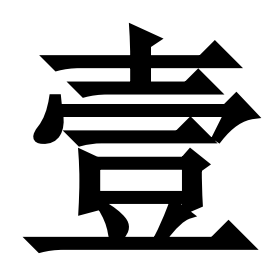

道可道, 非常道; 名可名, 非常名。

The Way that can be told of is not an Unvarying Way.

The names that can be named are not unvarying names. 
Cartilage is a connective tissue which in the body consists of 3 distinct types: hyaline cartilage, fibrocartilage and elastic cartilage [1]. The main matrix of cartilage consists of collagens and proteoglycans, which are essential for cartilage's compressive and tensile properties. Articular cartilage, responsible for the smooth joint movement, provides mechanical support to the joints and belongs to hyaline cartilage [2]. Chondrocytes are the only cell type present in articular cartilage and regulate the homeostasis of cartilage by synthesis of extracellular matrix. Multiple cytokines and signaling pathways have been shown to be involved in the regulation of chondrocyte homeostasis and cartilage development. Abnormal stimulation will force chondrocytes to change their normal functions and hence may contribute to the initiation and progression of joint diseases such as osteoarthritis (OA) and rheumatoid arthritis (RA) [3]. OA is a common chronic joint disease characterized by progressive degradation of articular cartilage, typical bone changes and signs of synovial inflammation especially in end stages of the disease. Millions people suffer from OA in the world, with symptoms of joint pain, stiffness, and as a consequence impaired mobility [4].

Due to its avascular and aneural features, cartilage has limited repair capacity once damaged. One of the most effective treatment strategies for such focal cartilage defects is cell therapy like autologous chondrocyte implantation (ACI) or more recently mesenchymal stem cell (MSC) based therapy [5-7]. In ACI, autologous chondrocytes are harvested from unaffected non-weight bearing regions of the traumatized joint, expanded in vitro, and then reimplanted into the damaged area [8]. Although ACI has been shown to successfully repair focal cartilage dsefects, this clinical procedure need further improvement due to some limitations. One major disadvantage of this method is the dedifferentiation of chondrocytes as a consequence of the required in vitro expansion of the cells in order to obtain sufficient cells for implantation. Dedifferentiated chondrocytes ultimately form fibrocartilage instead of hyaline cartilage after transplantation [9-12]. The mechanism underlying human chondrocyte dedifferentiation is still largely unknown. It has been reported that dedifferentiated chondrocyte can be redifferentiated in hyaline cartilage producing chondrocytes using three-dimensional (3-D) cell culture techniques such as pellet cultures, but this may not be completely successful due to the increased expression of markers for chondrocyte hypertrophy [13]. The latter may be a sign of 
premature cell death and transformation of the tissue into bone. Therefore, redifferentiation of chondrocytes, as well as preventing the hypertrophic phenotype are becoming increasingly appreciated.

Unlike most other tissues, chondrocytes have been adapted to low oxygen levels as a consequence the avasculare nature of cartilage tissue [14]. It has been shown that this adaptation to low oxygen levels play a very important role in chondrocyte biology. Indeed hypoxia is considered to have a positive influence on the healthy chondrocyte phenotype and on cartilage matrix formation $[15,16]$. In vivo, chondrocytes not only face a hypoxic environment, but particularly in disease are also exposed to an inflammatory environment. Proinflammatory cytokines like IL-1 $\beta$ potently drive OA progression by inducing the expression of Matrix Metallo Proteinases (MMPs) stimulating cartilage degradation in vitro and in vivo [17-19]. High levels in expression of inflammatory cytokines have been observed in OA plasma and in synovial fluid [20]. How the chondrocyte responds to a variety of stimuli in vivo under normal and pathological conditions is still largely unclear. Therefore, it is urgent to dissect the molecular mechanisms underlying chondrocyte dedifferentiation, redifferentiation and hypertrophic differentiation, to improve the application of human chondrocytes in cartilage tissue engineering and cell based therapies for the treatment of OA.

\section{Aims and Outlines of this Thesis}

The aim of this thesis is to elucidate the role of multiple signaling factors in the regulation of chondrogenic differentiation of human MSCs and in the redifferentiation of culture expanded and dedifferentiated human chondrocytes. There are several questions that are addressed in this thesis: i) how does the expression of important regulators of the WNT and BMP signaling pathways, like the WNT-antagonists DKK1 and FRZB and the BMP antagonist GREM1 change in human synovial fluid during OA progression (Chapter 3); ii) what is the role of Wnt signaling on chondrogenic differentiation of hMSCs (Chapter 4); iii) what is the effect of hypoxia on the chondrocyte's response to IL1 $\beta$, TGF- $\beta 3$ and/or BMP7 on chondrocyte redifferentiation (Chapter 5 and 6); iv) how can the growth factor BMP7 be utilized in tissue engineering (Chapter 7) and v) how can we use our knowledge to improve cell-based therapy 
in tissue regeneration.

Chapter 2 provides a comprehensive review about the regulatory role of signaling crosstalk in hypertrophy of chondrogenically differentiating MSCs and human articular chondrocytes.

OA is difficult to diagnose in an early stage of disease due to lack of obvious clinical symptoms and the insensitivity of routine radiography to detect loss of glycosaminoglycans in cartilage. Consequently the identification of diagnostic and/or prognostic biomarkers remains a big challenge. It has been reported that anabolic or catabolic molecules are released from joint tissue into synovial fluid and serum [21-23]. Previously we have identified the WNT antagonists Dickkopf-related protein 1 (DKK1), Frizzled-related protein (FRZB) and BMP antagonist Gremlin 1 as critical factors capable of inhibiting hypertrophic differentiation of chondrocytes [24]. We propose that their expression is changed in OA. Therefore, in Chapter 3, we have measured the concentration of DKK1, FRZB and GREM1 in synovial fluid and serum samples collected from end stage OA patients. Their expression is correlated with multiple OA features (macroscopically, histologically, and biochemically) and with the expression of inflammatory factors.

In chapter 4, we further study the role of canonical Wnt/ $\beta$-catenin signaling using small molecule agonists and antagonists in chondrogenic differentiation of hMSCs. Our findings suggest that fine tuning Wnt signal in the chondrogenic differentiation process of hMSC could contribute to the cartilage tissue engineering.

The maintenance of the permanent chondrocyte phenotype and the prevention of its dedifferentiation still is a big obstacle in ACI. Considering that native chondrocytes are exposed to hypoxia and are reimplanted in an inflamed joint, in Chapter 5 we investigated the influence of oxygen and inflammation separately and in combination on 3-D redifferentiation of healthy and OA chondrocytes. This data provides novel insights how oxygen tension influences the chondrocyte's response to pro-inflammatory cytokines. We provide evidence for a distinct role of the survival genes HIF $1 \alpha$ and HIF2 $\alpha$. 
Based on the findings in Chapter 5, we further explore the role of hypoxia combined with exogenous TGF- $\beta 3$ and/or BMP7 on chondrocyte redifferentiation in Chapter 6. We observe that the combination of TGF- $\beta 3$ and BMP-7 readjust the balance between catabolism and anabolism by upregulating anabolic activities and downregulating catabolic activities in OA chondrocytes. In Chapter 7, the potential application of BMP7 in tissue engineering is investigated. We present the selection and functional evaluation of highly specific single domain antibody fragments of heavy chain only antibodies of Camelidae (VHH) against bone morphogenetic protein 7 (BMP-7) and hydroxyapatite (HA). These two VHHs are genetically fused in a bifunctional VHH or bihead. We demonstrate the successful targeting of this bihead to bone upon injection in hairless mice. We also present evidence that the bihead successfully direct BMP7 to the bone. This study demonstrates the bihead VHH potential application in tissue engineering without modification of growth factor.

In chapter 8, the main conclusions of this thesis are discussed in a broader perspective. In addition, implications for improvement of current cell-based cartilage repair technology are discussed. 


\section{References}

1. $\quad$ El Sayed, K., et al., Heterotopic autologous chondrocyte transplantation--a realistic approach to support articular cartilage repair? Tissue Eng Part B Rev, 2010. 16(6): p. 603-16.

2. Morrison, E.H., et al., The development of articular cartilage: I. The spatial and temporal patterns of collagen types. J Anat, 1996. 189 ( Pt 1): p. 9-22.

3. Akkiraju, H. and A. Nohe, Role of Chondrocytes in Cartilage Formation, Progression of Osteoarthritis and Cartilage Regeneration. J Dev Biol, 2015. 3(4): p. 177-192.

4. Goldring, M.B., Chondrogenesis, chondrocyte differentiation, and articular cartilage metabolism in health and osteoarthritis. Ther Adv Musculoskelet Dis, 2012. 4(4): p. 269-85.

5. van der Kraan, P.M. and W.B. van den Berg, Chondrocyte hypertrophy and osteoarthritis: role in initiation and progression of cartilage degeneration? Osteoarthritis Cartilage, 2012. 20(3): p. 223-32.

6. Nazempour, A. and B.J. Van Wie, Chondrocytes, Mesenchymal Stem Cells, and Their Combination in Articular Cartilage Regenerative Medicine. Ann Biomed Eng, 2016. 44(5): p. 1325-54.

7. Richardson, S.M., et al., Mesenchymal stem cells in regenerative medicine: Focus on articular cartilage and intervertebral disc regeneration. Methods, 2016. 99: p. 69-80.

8. Brittberg, M., et al., Treatment of deep cartilage defects in the knee with autologous chondrocyte transplantation. N Engl J Med, 1994. 331(14): p. 889-95.

9. Cournil-Henrionnet, C., et al., Phenotypic analysis of cell surface markers and gene expression of human mesenchymal stem cells and chondrocytes during monolayer expansion. Biorheology, 2008. 45(3-4): p. 513-26.

10. Hubka, K.M., et al., Enhancing chondrogenic phenotype for cartilage tissue engineering: monoculture and coculture of articular chondrocytes and mesenchymal stem cells. Tissue Eng Part B Rev, 2014. 20(6): p. 641-54.

11. Peterson, L., et al., Two- to 9-year outcome after autologous chondrocyte transplantation of the knee. Clin Orthop Relat Res, 2000(374): p. 212-34. 
12. Roberts, S., et al., Autologous chondrocyte implantation for cartilage repair: monitoring its success by magnetic resonance imaging and histology. Arthritis Res Ther, 2003. 5(1): p. R60-73.

13. Benya, P.D. and J.D. Shaffer, Dedifferentiated chondrocytes reexpress the differentiated collagen phenotype when cultured in agarose gels. Cell, 1982. 30(1): p. 215-24.

14. Lafont, J.E., Lack of oxygen in articular cartilage: consequences for chondrocyte biology. Int J Exp Pathol, 2010. 91(2): p. 99-106.

15. Sheehy, E.J., C.T. Buckley, and D.J. Kelly, Oxygen tension regulates the osteogenic, chondrogenic and endochondral phenotype of bone marrow derived mesenchymal stem cells. Biochem Biophys Res Commun, 2012. 417(1): p. 305-10.

16. Hirao, M., et al., Oxygen tension regulates chondrocyte differentiation and function during endochondral ossification. J Biol Chem, 2006. 281(41): p. 31079-92.

17. Kobayashi, M., et al., Role of interleukin-1 and tumor necrosis factor alpha in matrix degradation of human osteoarthritic cartilage. Arthritis Rheum, 2005. 52(1): p. 12835.

18. Wojdasiewicz, P., L.A. Poniatowski, and D. Szukiewicz, The role of inflammatory and anti-inflammatory cytokines in the pathogenesis of osteoarthritis. Mediators Inflamm, 2014. 2014: p. 561459.

19. Tetlow, L.C., D.J. Adlam, and D.E. Woolley, Matrix metalloproteinase and proinflammatory cytokine production by chondrocytes of human osteoarthritic cartilage: associations with degenerative changes. Arthritis Rheum, 2001. 44(3): p. 585-94.

20. Sokolove, J. and C.M. Lepus, Role of inflammation in the pathogenesis of osteoarthritis: latest findings and interpretations. Ther Adv Musculoskelet Dis, 2013. 5(2): p. 77-94.

21. Sumer, E.U., et al., Application of biomarkers in the clinical development of new drugs for chondroprotection in destructive joint diseases: a review. Biomarkers, 2006. 11(6): p. 485-506.

22. Sandell, L.J. and T. Aigner, Articular cartilage and changes in arthritis. An introduction: cell biology of osteoarthritis. Arthritis Res, 2001. 3(2): p. 107-13.

23. Poole, A.R., et al., Changes in cartilage metabolism in arthritis are reflected by altered 
serum and synovial fluid levels of the cartilage proteoglycan aggrecan. Implications for pathogenesis. J Clin Invest, 1994. 94(1): p. 25-33.

24. Leijten, J.C., et al., Gremlin 1, frizzled-related protein, and Dkk-1 are key regulators of human articular cartilage homeostasis. Arthritis Rheum, 2012. 64(10): p. 3302-12. 


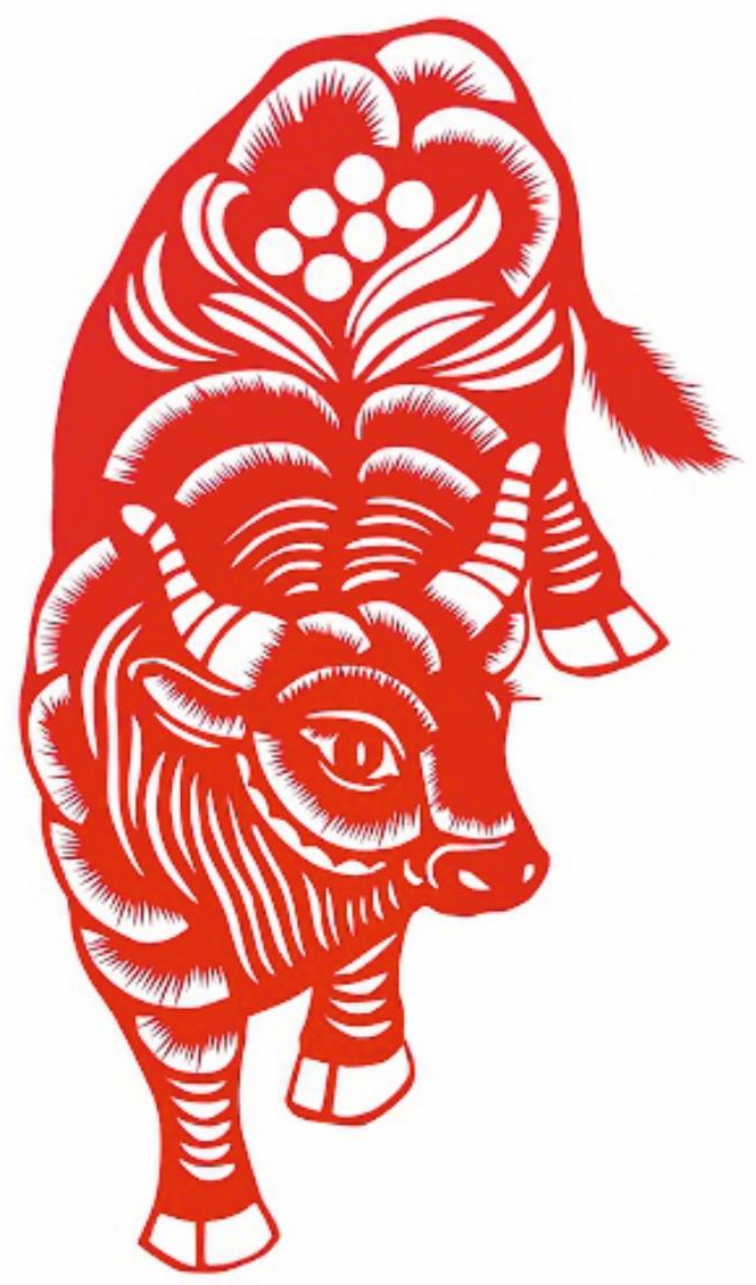




\section{Chapter 2}

\section{The regulatory role of signaling crosstalk in hypertrophy of MSCs and human articular chondrocytes*}

Leilei Zhong ${ }^{1 \#}$, Xiaobin Huang ${ }^{1,2 \#}$, Marcel Karperien ${ }^{1}$ and Janine N. Post

1 Developmental BioEngineering, MIRA Institute for Biomedical Technology and Technical Medicine, University of Twente, Enschede 7500 AE, The Netherlands

2 School of Life Sciences, Chongqing University, Chongqing 400030, China.

*Adapted from Zhong L, Huang X, Karperien M, Post JN. The Regulatory Role of Signaling Crosstalk in Hypertrophy of MSCs and Human Articular Chondrocytes. International journal of molecular sciences. 2015;16(8):19225-47. \# Leilei Zhong and Xiaobin Huang made equal contributions to this work.

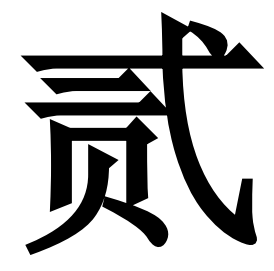

天下皆知美之为美, 斯恶已; 皆知善之为善, 斯不善已。

It is because every one under heaven recognizes beauty as beauty, that the idea of ugliness exists. And equally if every one recognized virtue as virtue, this would merely create fresh conceptions of wickedness. 


\begin{abstract}
Hypertrophic differentiation of chondrocytes is a main barrier in application of mesenchymal stem cells (MSCs) for cartilage repair. In addition, hypertrophy occurs occasionally in osteoarthritis (OA). Here we provide a comprehensive review on recent literature describing signal pathways in the hypertrophy of MSCs derived chondrocytes and chondrocytes, with an emphasis on the crosstalk between these pathways. Insight into the exact regulation of hypertrophy by the signaling network is necessary for the efficient application of MSCs for articular cartilage repair and for developing novel strategies for curing OA. We focused on articles describing the role of the main signaling pathways in regulating chondrocyte hypertrophy-like changes. Most researchers report hypertrophic differentiation in chondrogenesis of MSCs, and in both human OA and experimental OA. Chondrocyte hypertrophy is not under the strict control of a single pathway but appears to be regulated by an intricately regulated network of multiple signaling pathways, such as WNT, Bone morphogenetic protein (BMP)/Transforming growth factor- $\beta$ (TGF $\beta$ ), Parathyroid hormonerelated peptide (PTHrP), Indian hedgehog (IHH), Fibroblast growth factor (FGF), Insulin like growth factor 1 (IGF) and Hypoxia-inducible factor (HIF). This comprehensive review describes how this intricate signaling network influences tissue-engineering applications of MSCs in articular cartilage (AC) repair, and improves understanding the disease stages and cellular responses within an OA articular joint.
\end{abstract}

Keywords: chondrocytes; articular cartilage; signaling; signal crosstalk; hypertrophy; review; osteoarthritis; mesenchymal stem cells; chondrogenesis. 


\section{Introduction:}

Osteoarthritis (OA) is a multifactorial complex and chronic disease characterized by progressive degradation of joint cartilage. The underlying molecular mechanisms involved in the pathogenesis and progression of OA are still largely unknown, and currently no diseasemodifying therapy is available for OA.

In cell-based cartilage regeneration therapies, the use of mesenchymal stem cells (MSCs) has showed promising results. Evidence showed that MSCs can be differentiated into chondrocytes (marked by Sex determining region Y box 9 (SOX9); Aggrecan (ACAN); Collagen type II (Col2A1)) after a condensation state (marked by Cyclic adenosine monophosphate (cAMP), Transforming growth factor- $\beta$ (TGF $\beta$ ), Fibronectin, Neural cell adhesion molecule (N-CAM) and N-cadherin) in vivo and in vitro [1-3] (Fig. 1a). However, in the application of human MSCs for cartilage repair in vivo, hypertrophic differentiation towards the osteogenic lineage is observed. Prevention of hypertrophy is becoming increasingly important for clinical application of MSCs in cartilage tissue engineering [1, 4]. Interestingly, recent data indicate that the healthy chondrocyte phenotype switches toward a hypertrophic phenotype in degenerated cartilage [4-6]. Phenomena such as proliferation of chondrocytes, hypertrophic differentiation of chondrocytes, remodeling and mineralization of the extracellular matrix (ECM), invasion of blood vessels and apoptotic death of chondrocytes correspondingly also occur during OA [7]. In addition, transgenic mouse models have shown that deregulated hypertrophic differentiation of articular chondrocytes may be a driving factor in the onset and progression of OA [4]. Therefore, control of hypertrophic differentiation can be exploited as an effective strategy for cartilage repair, and used in bone regeneration, where hypertrophic cartilage could act as a template for endochondral bone formation [1]. However, the exact molecular mechanism underlying hypertrophic differentiation is not understood. Despite numerous studies about the function of single signaling pathways in hypertrophy, studies which explore comprehensive signaling pathways in hypertrophic differentiation of MSCs and chondrocytes have not been published in recent years. Here we discuss how signaling pathways are involved in hypertrophy of MSCs and chondrocytes, how these signaling pathways interplay, and how signal factors changed in OA disease. 


\section{Hypertrophy in chondrogenesis of MSCs in vitro}

MSCs are promising candidate cells for cartilage tissue engineering, as they are present in large quantities in adipose tissue, bone marrow, synovium and cartilage [8] and can be expanded for a number of passages without losing their ability to undergo chondrogenic differentiation. Unfortunately, the phenotype of MSCs in cartilage repair is unstable [9, 10]. The expression of cartilage hypertrophy markers (e.g. collagen type X) by MSCs undergoing chondrogenesis, raises concern for a tissue engineering application of MSCs, since chondrocyte hypertrophy in neocartilage could ultimately lead to apoptosis and ossification [11].

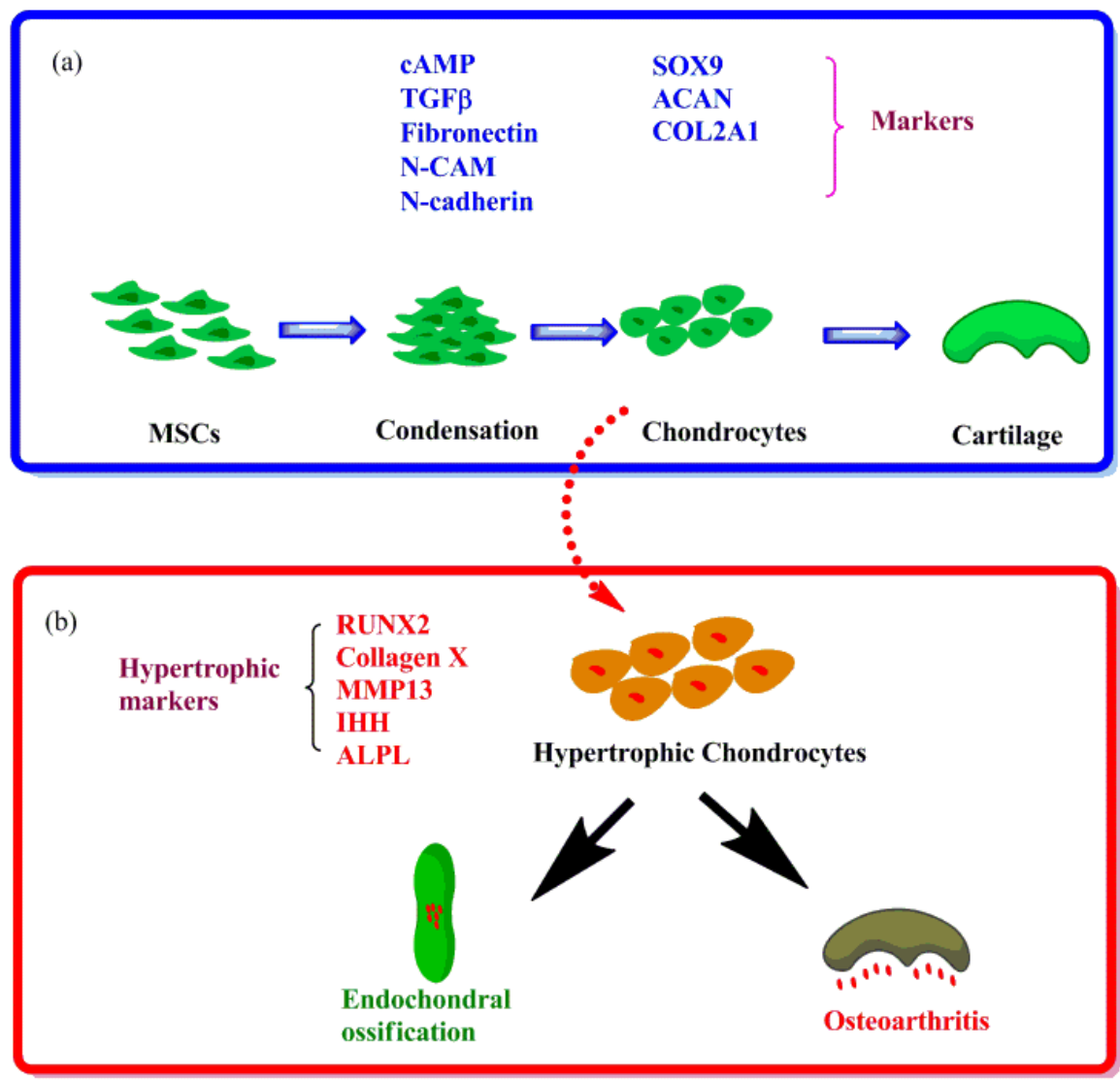

Figure 1 Chondrogenesis of MSCs and hypertrophic differentiation. (a) Chondrogenesis is initiated by the condensation of MSCs, and cell-cell contact. The expression of cAMP, TGF $\beta$, Fibronectin, N-CAM and N-cadherin is involved in this process and these factors are necessary for chondrogenic induction, marked by the expression of chondrogenic genes: SOX9, ACAN and COL2A1. Mature chondrocytes begin secreting cartilage matrix primarily consisting of 
collagen II and GAGs, which are the main components of cartilage; (b) Chondrocytes from in vitro chondrogenesis of MSCs or in vivo cartilage could undergo hypertrophic differentiation, which is characterized by an increase in cell volume and the expression of hypertrophic markers (RUNX2, Collagen X, MMP13, IHH and ALPL). In vivo, physiological endochondral ossification and pathological osteoarthritis could be initiated after remodeling, mineralization of the extracellular matrix, and apoptotic death of chondrocytes.

(a) Normal Chondrocyte

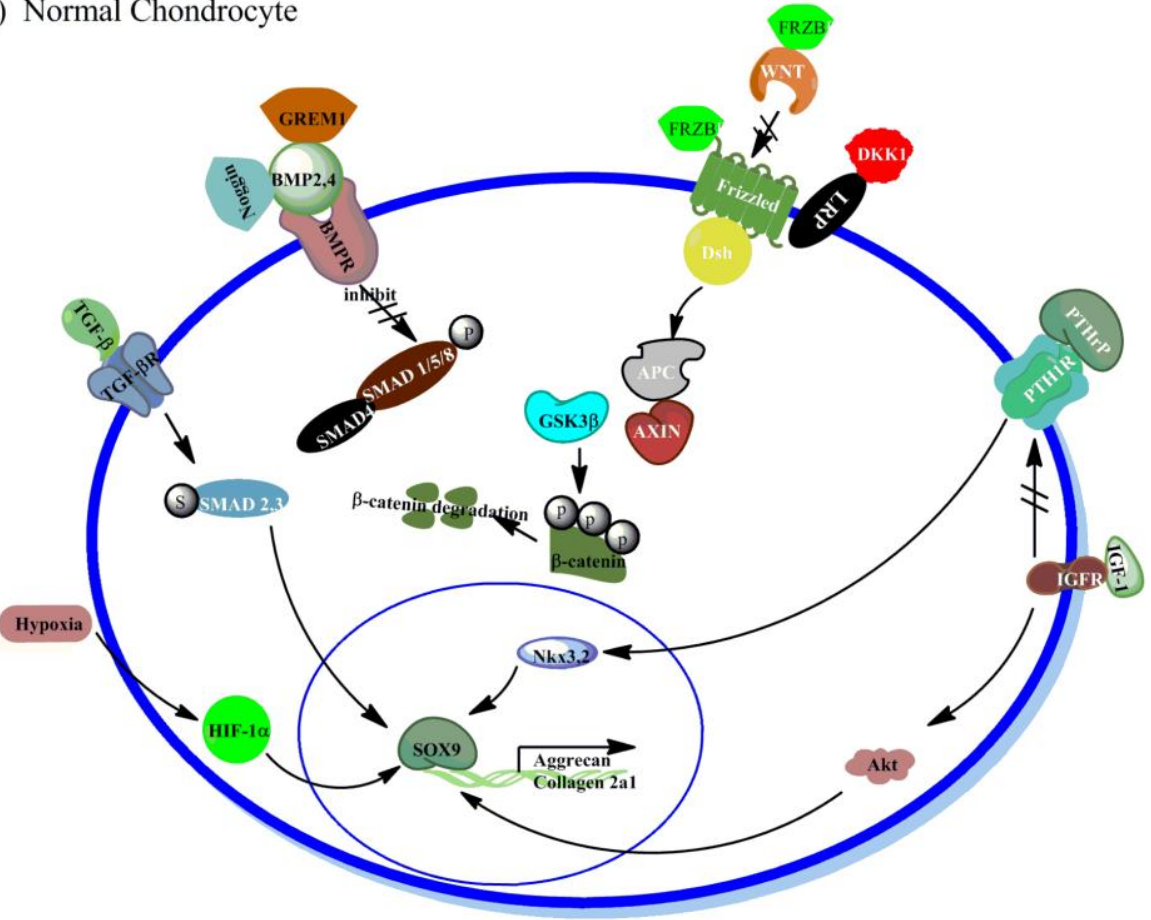

(b) Hypertrophic Chondrocyte

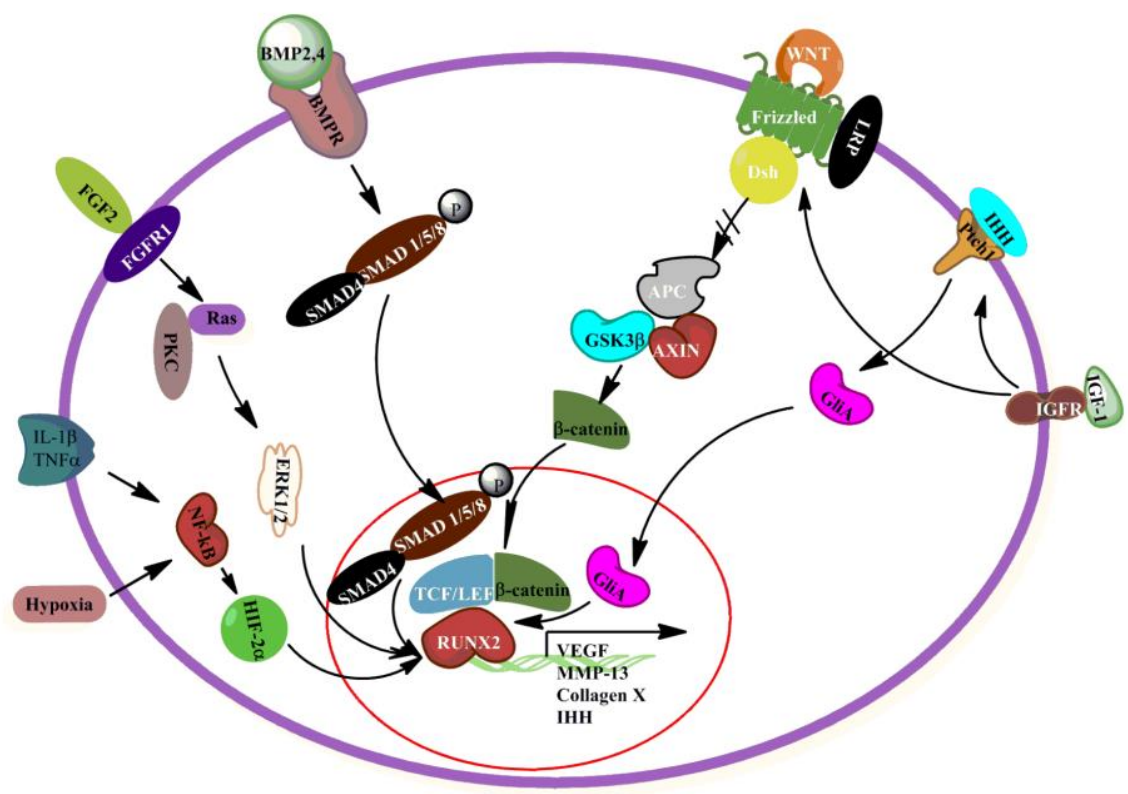


Figure 2 Signal pathways of chondrocyte hypertrophy. (a) In normal chondrocytes, signal pathways like WNT, BMP, IHH, etc. are regulated by their antagonists (DKK1 and FRZB for WNT, GREM1 for BMP) or other signal factors to get a fine balance to maintain the chondrocyte normal phenotype. The most important transcription factor regulating chondrocytes is SOX9, which is responsible for the expression of main chondrocyte makers including collagen type II and aggrecan. Striked through arrows indicate that the signaling pathway is inhibited by its antagonists; (b) In hypertrophic chondrocytes, signal pathways, such as WNT, BMP, IHH, etc. are deregulated by their inhibitors or other signal factors, which consequently leads to overexpression of these pathways. Subsequently, the effects of cascade pathways result in activating the transcription factor RUNX2, which regulates the transcription of hypertrophic markers like collagen X, MMP-13, VEGF and IHH.

\section{Hypertrophy in articular chondrocytes during OA progression}

Studies have shown that the development of OA may be caused by activation of hypertrophic differentiation of articular chondrocytes [12]. As Figure 1 shows, during hypertrophic differentiation of chondrocytes in OA, chondrocytes lose the stable phenotype and the expressions of Runt-related transcription factor 2 (RUNX2), Collagen type X, Matrix metalloproteinase 13 (MMP13), Indian hedgehog (IHH) and Alkaline phosphatase (ALPL) are detected [13]. Healthy articular cartilage (AC) is a stable tissue that has the potential to resist hypertrophic differentiation and maintain the normal phenotype through an unknown mechanism [14]. The interplay of multiple signaling pathways regulates the fate of chondrocytes, i.e. to remain within cartilage or to undergo hypertrophic differentiation.

\section{Signaling pathways in hypertrophy}

Multiple signaling pathways have been involved in regulation of hypertrophy-like changes in chondrogenesis of MSCs and chondrocytes. Based on recent literatures, the most important related pathways are WNT, Bone morphogenetic protein (BMP) / TGF $\beta$, Parathyroid hormone-related peptide (PTHrP), IHH, Fibroblast growth factor (FGF), Insulin like growth factor 1 (IGF) and Hypoxia-inducible factor (HIF) signaling pathways [15], Figure 2. In each single pathway, several distinct subtypes are involved in the regulation of chondrocyte differentiation and hypertrophy, Table 1. 


\begin{tabular}{|c|c|c|}
\hline Signal & Subtypes & Main Functions \\
\hline \multirow{8}{*}{ WNT } & WNT3a & Promotes chondrogenic differentiation; delays chondrocyte hypertrophy \\
\hline & WNT4 & Blocks chondrogenic differentiation; promotes chondrocyte hypertrophy \\
\hline & WNT5a & Promotes chondrogenic differentiation; delays chondrocyte hypertrophy \\
\hline & WNT5b & Promotes chondrogenic differentiation; delays chondrocyte hypertrophy \\
\hline & WNT8 & Blocks chondrogenic differentiation; promotes chondrocyte hypertrophy \\
\hline & WNT9a & Blocks both chondrogenic differentiation and chondrocyte hypertrophy \\
\hline & WNT11 & $\begin{array}{l}\text { Promotes chondrogenic differentiation; stimulates RUNX2 and IHH } \\
\text { expression }\end{array}$ \\
\hline & WNT16 & Upregulation is accompanied by the downregulation of FRZB \\
\hline \multirow{6}{*}{ BMP/TGF- $\beta$} & BMP2 & Induces chondrocyte hypertrophy \\
\hline & BMP4 & Induces chondrocyte hypertrophy \\
\hline & BMP7 & Maintain chondrogenic potential and prevents chondrocyte hypertrophy; \\
\hline & TGF- $\beta$ & Promotes chondrogenic differentiation; inhibits chondrocyte hypertrophy \\
\hline & PTHrP & $\begin{array}{l}\text { Blocks hypertrophy by stimulating Nkx3.2 and prevent RUNX2 } \\
\text { expression }\end{array}$ \\
\hline & $\mathrm{IHH}$ & $\begin{array}{l}\text { Promotes chondrocyte hypertrophy; } \\
\text { Stimulates proliferating chondrocytes to produce PTHrP }\end{array}$ \\
\hline \multirow{4}{*}{ FGF } & FGF2 & Promotes expression of RUNX2 \\
\hline & FGF8 & $\begin{array}{c}\text { Catabolic mediator with a pathological role in rat and rabbit articular } \\
\text { cartilage }\end{array}$ \\
\hline & FGF9 & Promotes chondrocyte hypertrophy \\
\hline & FGF18 & $\begin{array}{l}\text { Promotes chondrocyte proliferation and differentiation } \\
\text { in the early stages of cartilage development }\end{array}$ \\
\hline IGF & IGF-1 & $\begin{array}{l}\text { Promotes chondrocyte proliferation and maturation; } \\
\text { augments chondrocyte hypertrophy }\end{array}$ \\
\hline \multirow[t]{2}{*}{ HIF } & HIF-1 $\alpha$ & $\begin{array}{l}\text { Potentiates BMP2-induced SOX9 expression and cartilage formation, } \\
\text { while inhibiting RUNX2 expression and endochondral ossification }\end{array}$ \\
\hline & HIF-2 $2 \alpha$ & Increases expression of collagen X, MMP13 and VEGF \\
\hline
\end{tabular}

Table 1 The subtypes involved in multiple signal pathways (WNT, BMP/TGF $\beta$, PTHrP, IHH, FGF, IGF and HIF) and their main functions in the regulation of chondrocyte differentiation and hypertrophy.

\section{WNT signaling}

WNT signaling pathways are highly evolutionarily conserved pathways with crucial roles in embryonic development, patterning, tissue homeostasis, growth, as well as in the onset and 
progression of a variety of diseases [16]. There are three distinct intracellular signaling cascades well known so far: the canonical Wnt/ $\beta$-catenin pathway, the c-Jun $\mathrm{N}$-terminal kinase (JNK) pathway, and the $\mathrm{Wnt} / \mathrm{Ca} 2+$ pathway [17]. The canonical $\mathrm{Wnt} / \beta$-catenin pathway is the most-elucidated pathway, mediated by $\beta$-catenin accumulation in nucleus, having strong correlation with chondrocyte hypertrophy. As shown in Figure $2 b$, in most cases, the presence of Wnts that bind to the Wnt receptor Frizzled, results in formation complex of Adenomatous polyposis coli protein (APC), Glycogen synthase kinase $3 \beta$ (GSK3 $\beta$ ) and Axis inhibitor (AXIN), which leads to the release of $\beta$-catenin from the complex, followed by $\beta$-catenin accumulating in the cytoplasm, and then translocating into the nucleus. There $\beta$-catenin forms a complex with T cell-specific factor (TCF)/lymphoid enhancer binding protein (LEF) transcription factors to activate the transcription of target genes [17]. However, in the absence of a Wnt ligand, $\beta$-catenin is phosphorylated by the destruction complex and subsequently ubiquitinylated and targeted for proteasomal degradation.

Numerous studies have revealed a central role of WNT signaling in cartilage homeostasis. In cartilage, moderate activity of WNT is essential for chondrocyte proliferation and maintenance of their typical characteristics [18], but excessive activity increases chondrocyte hypertrophy and expression of cartilage degrading metalloproteinases [19]. For example, the conditional activation of the $\beta$-catenin gene in articular chondrocytes in adult mice leads to premature chondrocyte differentiation with collagen type $\mathrm{X}$ expression and the development of an OA-like phenotype [20]. However, ablation of $\beta$-catenin in the superficial zone of articular cartilage also strongly increases the expression of aggrecan and collagen type X [18]. SOX9 is the master transcription factor and thus a typical marker of chondrocytes, while RUNX2 usually is expressed highly in hypertrophic chondrocytes. This hypertrophy may be induced by the LEF/TCF/ $\beta$-catenin complex promoting RUNX2 expression in the redundant WNT signal pathway [21]. Much evidence has shown that the switch between SOX9 and RUNX2 expression determines the progression of mature chondrocytes into hypertrophy in response to canonical WNT signaling [17, 22-24].

There are several types of Wnt ligands, which play different roles in the chondrogenic differentiation and cartilage development. Experiments using retroviral misexpression in vivo and overexpression methods in vitro suggest distinct roles of different Wnts in the control of 
chondrogenic differentiation and hypertrophy. Wnt4 and Wnt8 block chondrogenic differentiation but promote hypertrophy [25, 26]. Wnt9a blocks both chondrogenic differentiation and hypertrophy [27]. Wnt3a and Wnt5b promote chondrogenic differentiation but delay hypertrophy [26]. The overexpression of Wnt11 in MSCs during chondrogenic differentiation promotes chondrogenesis and stimulates RUNX2 and IHH expression [28]. Wnt16 transient expression was found associated with the activation of the canonical Wnt pathway, and was present in the early phases of osteoarthritis, its upregulation was accompanied by the downregulation of the secreted WNT inhibitor Frizzled-related protein (FRZB)[29]. Wnt5a exhibit dual functions during chondrogenesis of MSCs. At early stages, Wnt5a induces chondrogenesis and hypertrophy through intracellular calcium release via Gprotein coupled receptor (GPCR) activation [1]. At later stage, it can act as an inhibitor of hypertrophy by activating the phosphoinositide 3-kinase (PI3K)/protein kinase B (PKB or Akt)-dependent pathway, which in-turn activates nuclear factor kappa-light chain-enhancer of activated B cells (NF- $\mathrm{B}$ ), an inhibitor of RUNX2 [30].

Interestingly, the expression of hypertrophy-related markers in chondrogenesis of MSCs is decreased in the presence of Dickkopf (DKK1), which acts as WNT signaling inhibitor (antagonist) by binding to low density lipoprotein receptor related protein (LRP5/6) through cartilage protective mechanisms [4]. Actually, DKK1, FRZB and Gremlin 1 (GREM1) are regarded as natural brakes on hypertrophic differentiation of articular cartilage [4]. Our studies also found increased hypertrophic differentiation and mineralization and decreased expression of chondrocyte markers in the absence of the WNT inhibitors DKK1 and FRZB during chondrogenesis of hMSCs. In MSC pellet cultures, the inhibition of canonical WNT by DKK1 and FRZB increased the expression of collagen II and aggrecan, but did not affect collagen X expression [25, 31, 32]. However, the reduction of WNT antagonist secreted frizzled related protein 1 (Sfrp1) in MSCs correlated with an increased amount of cytoplasmic $\beta$-catenin and an up-regulation of RUNX2 [33]. 


\section{Bone morphogenetic protein (BMP)/Transforming growth factor- $\beta$ (TGF $\beta$ ) Signaling}

\section{BMP signaling}

BMPs are multi-functional cytokines that belong to the transforming growth factor- $\beta$ (TGF- $\beta$ ) superfamily. BMP signaling is mediated primarily through the canonical BMP-Smad pathway in chondrocytes [34]. The pathway will be activated when BMPs bind to receptors BMPR-I and II phosphorylates Sma and Mad related proteins (Smad) 1, Smad5, and Smad8 (R-Smads). The R-Smadss form complexes with Smad4 and translocate into the nucleus, where they bind to regulatory regions of target genes to regulate their expression [35]. BMP has multiple roles during embryonic skeletal development, in addition to mesenchymal condensation and chondrogenic differentiation of MSCs, BMPs induce early cartilage formation [36] and are crucial local factor for chondrocyte proliferation and maturation in endochondral ossification $[37,38]$.

Although BMPs have a protective effect in articular cartilage, they are also involved in chondrocyte hypertrophy and matrix degradation [39-42]. It was reported that the BMP signaling pathway was primarily activated during fracture healing via endochondral ossification and was detected in hypertrophic chondrocytes [43]. Steinert et al. showed that BMP2 and BMP4 induce hypertrophy during the chondrogenic differentiation of human MSCin vitro [37]. In another study, BMP2 was found to induce chondrocyte hypertrophy during chondrogenesis of progenitor cells ATDC5, whereas BMP-7 appeared to increase or maintain chondrogenic potential and prevent chondrocyte hypertrophy [44]. In vivo studies showed that overexpression of BMP4 in cartilage of transgenic mice resulted in an increased hypertrophic zone, indicating increased differentiation of hypertrophic chondrocytes [45]. As the BMPs also played role in the skeletal development, it may be that BMPs will drive the chondrocytes to form bone after ossification, rather than to remain as articular chondrocytes [46]. Therefore, BMPs can be protective for articular cartilage but may have harmful effects on $\mathrm{AC}$ by inducing chondrocyte terminal differentiation and contributing to OA progression [31]. Our previous study has shown that addition of GREM1, the inhibitor of BMP signaling was able to slow down the hypertrophic differentiation and decrease the mineralization in the process of chondrogenesis of hMSCs [4]. In addition, another BMP inhibitor, Noggin, can block thyroid-induced hypertrophy by inhibiting BMP4 during MSC's chondrogenesis [47]. 


\section{TGF- $\beta$ signaling}

TGF $\beta$ is a potent inducer of chondrogenesis in vitro [48, 49]. During chondrogenesis of MSCs, TGF $\beta$ is the main initiator of MSC condensation. After aggregation, TGF $\beta$ signaling further stimulates chondrocyte proliferation while inhibits chondrocyte hypertrophy and maturation [50-55]. Conversely, the activation of the Smad1/5/8 pathway is able to stimulate hypertrophic differentiation with the consequent expression of the hypertrophic markers collagen X, MMP13 and ALPL during chondrogenesis of MSCs [56]. Although TGF $\beta$ is clearly crucial in inhibiting chondrocyte hypertrophy during early phases of mesenchymal condensation and chondrocyte proliferation, its addition to chondrocyte differentiation medium in pellet cultures of MSCs was not sufficient to suppress the onset of hypertrophy [9$11]$.

Most recently, several lines of evidence have suggested that the TGF $\beta /$ Smad pathway played a critical role in the regulation of articular chondrocytes hypertrophy and maturation during OA development [57-59]. Zuscik and colleagues have shown that treatment of articular chondrocytes with 5-azacytidine (5azaC), an anti-tumor agent that functions by blocking DNA methylation, resulted in a shift of regulatory dominance from maturation suppression via TGF $\beta$ signaling to maturation acceleration by BMP-2 signaling[60], which confirm that a shift in signaling dominance from TGF $\beta$ to BMP is sufficient to induce AC maturation [60]. This study also raised a possibility that a similar shift in signaling dominance occurs when these cells progresses inappropriately, such as in osteoarthritis, where the balance between TGF $\beta$ and BMP signaling pathways may be broken. It has been suggested that TGF $\beta$ inhibit terminal hypertrophic differentiation of chondrocyte and maintain normal articular cartilage through Smad $2 / 3$ signals $[58,61]$. The Smad3 pathway can be activated by TGF- $\beta$ directly to stablize Sox 9 transcription complex and inhibit RUNX2 expression through epigenetic regulation [62, 63]. Homozygous mutant mice of targeted disruption of Smad3- exon 8 developed degenerative joint disease resembling human OA, characterized by progressive loss of articular cartilage, and abnormally increased numbers of collagen type X expressing chondrocytes in synovial joints [58]. However, TGF $\beta 1$ administration has been shown to redirect expanded human articular chondrocytes towards hypertrophy [64]. Moreover, TGF $\beta$ can induce synovial lining cells to produce inflammatory factors, such as IL1 $\beta$ and TNF $\alpha$, which further stimulate articular chondrocytes terminal hypertrophy, depositing collagen type $X$ instead of collagen type II and aggrecan [65]. It have been reviewed the TGF- $\beta$ 
superfamily and its downstream phosphorylation of Smads exhibit both stimulatory and inhibitory effects on chondrocyte hypertrophy [66].

\section{The crosstalk between BMP/TGF $\beta$ and WNT signaling in regulating hypertrophy}

It has been report $\beta$-catenin crosstalk with TGF $\beta$ in regulating hypertrophy of MSCs [67]. In the process of TGF $\beta$-induced chondrogenesis of MSCs, temporal activation of $\beta$-catenin leaded to enhanced chondrogenic induction, further developed into hypertrophy and mineralization phenotype in vivo. However, the continuous co-activation of two signaling pathways resulted in hypertrophy inhibition, characterized by the suppressed expression of collagen type X, RUNX2, and ALPL, and did not lead to ossified tissue in vivo [67].

It is demonstrated that the crosstalk between WNT and BMP play key roles in regulating chondrocyte activity in pathogenesis of osteoarthritis, which may be cell type-specific [68]. Papathanasiou and colleagues reported the function and crosstalk between BMP2 and canonical Wnt/ $\beta$-catenin signaling in regulating chondrocyte hypertrophy and matrix metalloproteinase (MMP)/aggrecanolytic ADAMTS (a disintegrin like and metalloproteinase with thrombospondin type I motif) synthesis in OA [69]. In this study, they showed human end-stage OA chondrocytes can produce BMP2 and BMP4. Interestingly, only BMP2, but not BMP4, can drive the expression of low-density lipoprotein receptor 5 (LRP5), which is one of most important co-receptors for WNT signaling that leads to $\beta$-catenin stabilization, accumulation, nuclear translocation, and activation of target genes. It can be concluded that the BMP-2-induced Wnt/ $\beta$-catenin signaling pathway activation through LRP-5 induces chondrocyte catabolic action and hypertrophy [69].

This report adds to the accumulating evidence that increased or excessive activation of canonical WNT signaling in chondrocytes is detrimental and contributes to OA cartilage degradation. Recently, studies from our group also indicated that the natural WNT and BMP antagonists DKK1, FRZB and GREM1 inhibit hypertrophic differentiation of hMSCs during chondrogenesis by blocking WNT and BMP pathways [4]. Therefore therapeutic approaches to block or suppress canonical WNT and BMP2 pathways using their natural antagonists may protect cartilage damage in end-stage OA. 


\section{Parathyroid hormone-related peptide (PTHrP)/ Indian hedgehog (IHH)}

PTHrP is a member of the parathyroid hormone (PTH) family that blocks hypertrophy by stimulating NK3 homeobox 2 (Nkx3.2) [70] and preventing RUNX2 expression [71]. Huang supposed SOX9 is a target of PTHrP signaling in the growth plate and that the increased activity of SOX9 might mediate the effect of PTHrP in maintaining the cells as nonhypertrophic chondrocytes [72]. IHH is an important factor that involved in endochondral ossification and expressed in prehypertrophic chondrocytes [73]. In IHH knockout mice, the proliferation and hypertrophy of chondrocytes are significantly reduced [74]. Evidence shown that IHH can positively regulate the transcription and expression of collagen type $\mathrm{X}$ via Runx2/Smad interactions through downstream transcription factors GLI-Kruppel family members (Gli) 1/2 [75]. Both IHH and PTHrP signaling play crucial roles in regulating the onset of chondrocyte hypertrophy. Vortkamp and colleagues [76] found that IHH stimulated proliferating chondrocytes to produce PTHrP, which in turn accelerated the proliferation of periarticular cells and prevented the onset of chondrocyte hypertrophy, thereby keeping chondrocytes in a proliferating state. This negative feedback loop regulates the balance between proliferation and maturation of chondrocytes, ensuring orderly bone formation [76]. On the other hand, resting chondrocytes at the ends of long bones secrete PTHrP, subsequently suppressing IHH production in the proliferating zone. Chondrocytes outside of this paracrine signaling range produce IHH and undergo hypertrophy [1]. PTHrP forms a feedback loop with IHH to regulate the proliferation and onset of hypertrophic differentiation [77-79]. During endochondral bone formation, PTHrP-dependent IHH signaling inhibiting chondrocyte hypertrophy is dominant, thereby obscuring the promoting effect of PTHrPindependent IHH signaling. Other researchers reported that IHH can also function independently of PTHrP to promote chondrocyte hypertrophy [80].

In PTHrP knock out mice, the absence of PTHrP caused diminished chondrocytes and accelerated hypertrophic differentiation, and led to premature mineralization of extracellular matrix and apoptosis [76, 81]. However, targeted overexpression of PTHrP under the control of the cartilage-specific collagen type II promoter resulted in the opposite effect of chondrodysplasia through delay of the terminal differentiation of chondrocytes, inhibition of apoptosis and disruption of endochondral ossification [82]. A co-culture model from Jiang 
and colleagues [83] demonstrated that in healthy articular cartilage PTHrP, secreted by chondrocytes from surface layers, inhibits the hypertrophic potential of chondrocytes residing in the deep layer so as to maintain the homeostasis of articular cartilage, but the effect was not confirmed in vivo. In another cell study, it was proved that PTHrP from human articular chondrocytes inhibits hypertrophy of MSCs during chondrogenesis in co-culture, and intermittent supplementation of PTHrP also improve chondrogenesis of MSCs and reduce the hypertrophy $[84,85]$. Similar phenomenon was observed in MSCs pellet studies, it was showed that PTHrP treatment leads to suppression of hypertrophy but also down-regulates collagen II [49]. But when cultured under hypertrophy-enhancing conditions, PTHrP could not diminish the induced enhancement of hypertrophy in the MSC pellets [86]. However, other researchers observed a selective hypertrophic inhibition upon PTHrP treatment with stable or even up-regulated expression of collagen II [87, 88]. This discrepancy might be linked to the existence of both PTHrP receptor 1(PTH1R)-dependent and PTH1Rindependent pathways [1]. PTH1R knockout mice showed accelerated hypertrophy and were unaffected by treatment with PTHrP, indicating that the inhibition on hypertrophy is dependent on PTH1R receptor binding [89]. The choice of the PTHrP isoform has further been shown to affect the suppressive action on hypertrophy, with isoform 1-34 being the most effective in promotion of chondrogenesis as well as inhibition of hypertrophy [90].

Cell studies have shown that FGF2 combined with PTHrP inhibited the TGF $\beta$ responsive COL2A1 and COL10A1 expression and ALPL induction. However, calcification of implanted pellets was not prevented by PTHrP in vivo [49]. In another study, the combined delivery of TGF- $\beta 3$ and PTHrP in nude mice reduced calcification [91]. In addition, the canonical Wnt pathway is known to promote chondrocyte hypertrophy via inhibition of PTHrP signaling activity [92]. Therefore, PTHrP repress hypertrophic cartilage differentiation whereas WNT and IHH promote hypertrophy of chondrocytes. Hence, the fine balance of the crosstalk between signal pathways is a requirement for the normal phenotype of chondrocytes.

\section{Fibroblast growth factor (FGF) Signaling}

FGF signaling plays a critical role in controlling chondrocyte differentiation [93]. Specifically, four members of the fibroblast FGF family, FGF2, FGF8, FGF9 and FGF18, have been implicated as contributing factors in cartilage homeostasis [93-97]. FGF2 has been 
shown to be expressed in proliferating and prehypertrophic chondrocytes, periosteal cells and osteoblasts[98]. In human articular chondrocytes, the binding of FGF2 to FGFR1 activates Ras and Protein kinase $\mathrm{C}$ delta $(\mathrm{PKC} \delta)$, which transfer the signals into the nucleus to positively regulate the expression of RUNX2 by the Raf-MEK1/2-ERK1/2 cascade[99]. Under experimental OA conditions, FGF-8 has been identified as a catabolic mediator with a pathological role in rat and rabbit articular cartilage [100]. However, little is known about the precise biological function of FGF-8 on human adult articular cartilage[97]. In developing stylopod elements, FGF9 promotes chondrocyte hypertrophy at early stages and regulates vascularization of the growth plate and osteogenesis at later stages of skeletal development. Fgf9-/- mice have normal limb bud development and mesenchymal condensations, but in stylopod elements show decreased chondrocyte proliferation, delayed initiation of chondrocyte hypertrophy and abnormal osteogenesis in skeletal vascularization [96]. In the early stage of cartilage development, FGF18 is expressed in the perichondrium and joint spaces to promote chondrocyte proliferation and differentiation. In Fgf18-/- mice, the phenomenon of delayed mineralization was observed, which was found to be closely associated with delayed initiation of chondrocyte hypertrophy, decreased chondrogenesis proliferation of early stages, delayed skeletal vascularization and delayed osteoclast and osteoblast recruitment to the growth plate [101]. Further studies have shown that FGF18 is necessary to induce VEGF expression by signaling to FGFR 1 and 2 in hypertrophic chondrocytes [101]. The FGF receptor 3 (FGFR3) is a tyrosine kinase receptor, expressed in proliferating chondrocytes and early hypertrophic chondrocytes in the growth plate. Both FGF9 and FGF18 are the major ligands of FGFR3 in the growth plate[102]. Recently, Shung and coworkers found that FGFR3 expression increases the expression of SOX9 and decreases $\beta$-catenin levels in cultured mesenchymal cells [103].

The interplay of WNT and FGF signaling is important to determine the fate of MSCs and their subsequent differentiation. FGFR1 appears to act downstream of the $\beta$-catenin pathway and serves as a key determinant in the lineage decision of skeletal precursors [104]. Hypertrophic maturation of chondrocytes is highly regulated by the interplay of the FGF, IHH, BMP, and WNT signaling pathways. More specifically, FGF signaling accelerates the speed of terminal hypertrophic differentiation, and acts in an antagonistic relationship with IHH expression [105]. Another study suggests that the FGF and BMP pathways collaborate to promote aspects of hypertrophic chondrocyte maturation [106]. However, cartilage of mice carrying a targeted deletion of Fgfr3 is characterized by increased regions of proliferating and 
hypertrophic chondrocytes [107]. A study from Weiss and colleagues also showed that FGF2, together with PTHrP, may inhibit chondrocyte hypertrophic differentiation and is therefore necessary to obtain stable chondrocytes [49].

\section{Insulin like growth factor (IGF) Signaling}

IGF-1 has been identified as an important growth factor for skeletal development by promoting chondrocyte proliferation and maturation, while inhibiting apoptosis to form bones with appropriate size and strength. IGF-1 transmit signals via the type 1 IGF-1 receptor (IGF1R), which is expressed in the proliferating and prehypertrophic zone chondrocytes of growth plate [108]. Evidence shown IGF-1 stimulates growth plate chondrocytes at all stages of differentiation [109]. High level of IGF-1 was detected in osteoarthritic human articular cartilage [110]. The local infusion of IGF-1 in rabbit tibial growth plate increased the numbers of both proliferative and hypertrophic chondrocytes and promoted hyperplasia of bony trabeculae within the epiphysis [111].

It has been shown that IGF-1 stimulates the chondrogenic differentiation of MSCs into chondrocytes, and into pre-hypertrophic and hypertrophic chondrocytes [112]. Recombinant adeno-associated virus (rAAV)-mediated IGF-I overexpression delayed terminal differentiation and hypertrophy in the newly formed cartilage, which may due to contrasting effects upon the osteogenic expression of RUNX2 and $\beta$-catenin [113]. Another study demonstrates that IGF-1 enhances chondrocyte hypertrophy by insulin-like actions, and terminal hypertrophic chondrocytes are reduced in Igf1 null mice [114]. Repudi's study found that WNT induced secreted protein 3 (WISP3) inhibits IGF-1 induced collagen $\mathrm{X}$ induction, reactive oxygen species (ROS) accumulation and ALPL activity, all of which are associated with the induction of chondrocyte hypertrophy [115]. In addition, Mushtaq also found that IGF-1 stimulated chondrocyte hypertrophy and reversed the growth-inhibitory dexamethasone effects in mouse metatarsal [116]. However, evidence shown chick embryo chondrocytes maintained their normal phenotype and were prevented to undergo hypertrophic differentiation in the presence of IGF-1 [117]. Clearly, the IGF-I mediated improvement in growth was performed by altering the balance between proliferating and hypertrophic chondrocytes. 
IGF-1 signaling also is involved in the interaction between the thyroid hormone and the Wnt/ $\beta$-catenin signaling pathways in regulating growth plate chondrocyte proliferation and differentiation. Evidence showed that IGF-1 and the IGF-1 receptor (IGF1R) stimulate Wnt-4 expression and $\beta$-catenin activation in growth plate chondrocytes. Chondrocyte proliferation and terminal differentiation induced by IGF-1/IGF1R can be partially inhibited by the Wnt antagonists FRZB and DKK1 [118]. The IGF-1/IGF1R signaling and IGF-1 dependent PI3K/Akt/GSK-3 $\beta$ signaling can be activated by $\mathrm{T}(3)$ in the growth plate, and the chondrocytes undergo proliferation and differentiation to prehypertrophy. It seems chondrocyte proliferation may be triggered by the IGF-1/IGF1R-mediated PI3K/Akt/GSK3 $\beta$ pathway, while cell hypertrophy is likely due to activation of $\mathrm{Wnt} / \beta$-catenin signaling, which is at least in part initiated by IGF-1 signaling or the IGF-1-activated PI3K/Akt signaling pathway [118]. The fact that IHH expression was reduced in Igf1-/- mice long bones, whereas expression of PTHrP was increased, suggested that IGF-1 signaling is also required to maintain the IHH-PTHrP loop during skeletogenesis [119].

\section{Hypoxia-inducible factor (HIF) signaling}

Healthy articular cartilage is a typical avascular tissue, and chondrocytes are able to survive in low oxygen environments [120]. Hypoxia is considered to be a positive influence on the healthy chondrocyte phenotype and cartilage matrix formation. A recent study from our group has shown that the articular cartilage-enriched gene transcripts of GREM1, FRZB, and DKK1, which are established inhibitors of hypertrophic differentiation, were robustly increased in chondrogenic hMSCs pellets under hypoxic conditions, whereas under normoxia conditions these genes did not increase markedly [121]. Envidence shown hypoxia enhances chondrogenesis and prevents terminal differentiation through a PI3K/Akt/FoxO dependent anti-apoptotic effect [122]. The hypoxic response is mainly mediated by HIF, which includes three family members, HIF- $1 \alpha,-2 \alpha,-3 \alpha$ [123], particularly HIF- $1 \alpha$ and HIF-2 $\alpha$, play an active role in chondrocyte development.

Under hypoxic conditions, the transcription factor HIF-1 $\alpha$ accumulates and activates the transcription of genes, which are involved in energy metabolism, angiogenesis, vasomotor control, apoptosis, proliferation, and matrix production. In subcutaneous stem cell implantation studies, HIF-1 $\alpha$ was shown to potentiate BMP2-induced SOX9 and cartilage formation, while inhibiting RUNX2 and endochondral ossification during ectopic 
bone/cartilage formation. In the fetal limb culture, HIF-1 $\alpha$ and BMP2 synergistically promoted the expansion of the proliferating chondrocyte zone and inhibited chondrocyte hypertrophy and endochondral ossification [124]. However, HIF-2 $\alpha$, encoded by Epas1, was identified as a regulator of endochondral bone formation, and appears to be a central positive regulator of collagen X, MMP13 and VEGF expression by enhancing promoter activities through specific binding to the hypoxia-responsive elements [125]. Inflammatory factors like IL- $1 \beta$ and TNF- $\alpha$ can increase the HIF- $2 \alpha$ expression by NF- $\kappa$ B signaling in chondrocytes $[125,126]$. Further experiments have shown HIF- $2 \alpha$ crosstalks with the $\beta$-catenin and NF- $\kappa \mathrm{B}$ pathways to promote chondrocyte apoptosis and endochondral ossification [127]. RUNX2 and IHH are identified as the possible transcriptional targets of HIF-2 $\alpha$ related to endochondral ossification, both of them are involved in the regulation of hypertrophic differentiation of chondrocytes $[125,128]$. The gene corresponding to nicotinamide phosphoribosyltransferase (NAMPT) is also a direct target of HIF-2 $\alpha$, and plays an essential catabolic role in $\mathrm{OA}$ pathogenesis and acts as a crucial mediator of osteoarthritic cartilage destruction caused by HIF-2 $\alpha$ or destabilisation of the medial meniscus (DMM) surgery [129]. There is evidence that HIF- $2 \alpha$ causes cartilage destruction by regulating crucial catabolic genes [126] and potentiating Fas-mediated chondrocyte apoptosis [130]. However, Lafont and coworkers found that hypoxia promotes cartilage matrix synthesis specifically through HIF-2 $\alpha$ but not HIF-1 $\alpha$ mediated SOX9 induction of key cartilage genes [131]. The seemingly conflicting effects of HIF-2 $\alpha$ to chondrocyte or cartilage could be induced through different pathways and the differences in experiments performed in vivo and in vitro, which need to be clarified. The balance between HIF-1 $\alpha / \mathrm{HIF}-2 \alpha$ activities clearly contributes to the control of cartilage homeostasis.

\section{Conclusions}

Chondrocyte differentiation is regulated by multiple signal transduction pathways. Maintaining a normal chondrocyte phenotype and avoiding hypertrophy is important for cartilage repair. SOX9 and RUNX2 are two typical markers in chondrocyte development. SOX9 is expressed in chondrocytes, while RUNX2 is highly expressed in hypertrophic chondrocytes. In most cases, a hypertrophic phenotype was accompanied by high expression of RUNX2 through activation of either of the WNT, BMP, IHH, FGF and HIF signaling pathways. However, TGF $\beta$, IGF-I and PTHrP promote the proliferation of chondrocytes. Here 
we propose a model in which the balance of these signal pathways adjusts the state of chondrocyte proliferation or hypertrophy through the shifting between SOX9 and RUNX2 transcriptional activities. In the WNT pathway, the LEF/TCF/ $\beta$-catenin complex can promote RUNX2 expression. BMP/TGF- $\beta$ signaling has a dual role in the chondrocyte development. TGF- $\beta$ induces collagen II and SOX9 deposition through Smad2/3 phosphorylation pathway, while BMP2/4 promotes chondrocyte hypertrophy and cartilage mineralization via Smad1/5/8 phosphorylation. PTHrP represses hypertrophic cartilage differentiation whereas IHH signaling positively regulates the hypertrophic phenotype by high transcription and expression of collagen type $\mathrm{X}$ and RUNX2. IGF-1 signaling stimulates chondrocyte proliferation by the IGF-1/IGF1R-mediated PI3K/Akt/GSK3 $\beta$ pathway, while cell hypertrophy is likely due to activation of Wnt/ $\beta$-catenin and IHH signaling by IGF-1. HIF- $1 \alpha$ and HIF- $2 \alpha$ have a distinct role in the chondrocyte development. The former inhibits the RUNX2 expression, while the latter enhances the expression of collagen X, MMP13 and RUNX2 and promotes the hypertrophic differentiation of chondrocytes. The fine balance of the crosstalk between these signaling pathways is a requirement for normal chondrocyte differentiation and cartilage development. 


\section{References}

1. Studer, D., et al., Molecular and biophysical mechanisms regulating hypertrophic differentiation in chondrocytes and mesenchymal stem cells. Eur Cell Mater, 2012. 24: p. 118-35; discussion 135.

2. Hall, B.K. and T. Miyake, All for one and one for all: condensations and the initiation of skeletal development. Bioessays, 2000. 22(2): p. 138-47.

3. Mrugala, D., et al., Gene expression profile of multipotent mesenchymal stromal cells: Identification of pathways common to TGFbeta3/BMP2-induced chondrogenesis. Cloning Stem Cells, 2009. 11(1): p. 61-76.

4. Leijten, J.C., et al., Gremlin 1, frizzled-related protein, and Dkk-1 are key regulators of human articular cartilage homeostasis. Arthritis Rheum, 2012. 64(10): p. 3302-12.

5. Goldring, M.B. and K.B. Marcu, Cartilage homeostasis in health and rheumatic diseases. Arthritis Res Ther, 2009. 11(3): p. 224.

6. Fosang, A.J. and F. Beier, Emerging Frontiers in cartilage and chondrocyte biology. Best Pract Res Clin Rheumatol, 2011. 25(6): p. 751-66.

7. Dreier, R., Hypertrophic differentiation of chondrocytes in osteoarthritis: the developmental aspect of degenerative joint disorders. Arthritis Res Ther, 2010. 12(5): p. 216.

8. Williams, R., et al., Identification and clonal characterisation of a progenitor cell sub-population in normal human articular cartilage. PLoS One, 2010. 5(10): p. e13246.

9. Sekiya, I., et al., In vitro cartilage formation by human adult stem cells from bone marrow stroma defines the sequence of cellular and molecular events during chondrogenesis. Proc Natl Acad Sci U S A, 2002. 99(7): p. 4397-402.

10. Pelttari, K., et al., Premature induction of hypertrophy during in vitro chondrogenesis of human mesenchymal stem cells correlates with calcification and vascular invasion after ectopic transplantation in SCID mice. Arthritis Rheum, 2006. 54(10): p. 325466.

11. Mueller, M.B. and R.S. Tuan, Functional characterization of hypertrophy in chondrogenesis of human mesenchymal stem cells. Arthritis Rheum, 2008. 58(5): p. 1377-88.

12. Hunter, D.J. and D.T. Felson, Osteoarthritis. Bmj, 2006. 332(7542): p. 639-42. 
13. van der Kraan, P.M. and W.B. van den Berg, Chondrocyte hypertrophy and osteoarthritis: role in initiation and progression of cartilage degeneration? Osteoarthritis Cartilage, 2012. 20(3): p. 223-32.

14. Mackie, E.J., et al., Endochondral ossification: how cartilage is converted into bone in the developing skeleton. Int J Biochem Cell Biol, 2008. 40(1): p. 46-62.

15. Kronenberg, H.M., Developmental regulation of the growth plate. Nature, 2003. 423(6937): p. 332-6.

16. Miller, J.R., The Wnts. Genome Biol, 2002. 3(1): p. Reviews3001.

17. Yano, F., et al., The canonical Wnt signaling pathway promotes chondrocyte differentiation in a Sox9-dependent manner. Biochem Biophys Res Commun, 2005. 333(4): p. 1300-8.

18. Yasuhara, R., et al., Roles of beta-catenin signaling in phenotypic expression and proliferation of articular cartilage superficial zone cells. Lab Invest, 2011. 91(12): p. 1739-52.

19. Blom, A.B., et al., Involvement of the Wnt signaling pathway in experimental and human osteoarthritis: prominent role of Wnt-induced signaling protein 1. Arthritis Rheum, 2009. 60(2): p. 501-12.

20. Zhu, M., et al., Activation of beta-catenin signaling in articular chondrocytes leads to osteoarthritis-like phenotype in adult beta-catenin conditional activation mice. J Bone Miner Res, 2009. 24(1): p. 12-21.

21. Dong, Y.F., et al., Wnt induction of chondrocyte hypertrophy through the Runx2 transcription factor. J Cell Physiol, 2006. 208(1): p. 77-86.

22. Akiyama, H., et al., Interactions between Sox9 and beta-catenin control chondrocyte differentiation. Genes Dev, 2004. 18(9): p. 1072-87.

23. Hill, T.P., et al., Canonical Wnt/beta-catenin signaling prevents osteoblasts from differentiating into chondrocytes. Dev Cell, 2005. 8(5): p. 727-38.

24. Tamamura, Y., et al., Developmental regulation of Wnt/beta-catenin signals is required for growth plate assembly, cartilage integrity, and endochondral ossification. J Biol Chem, 2005. 280(19): p. 19185-95.

25. Enomoto-Iwamoto, M., et al., The Wnt antagonist Frzb-1 regulates chondrocyte maturation and long bone development during limb skeletogenesis. Dev Biol, 2002. 251(1): p. 142-56.

26. Church, V., et al., Wht regulation of chondrocyte differentiation. J Cell Sci, 2002. 115(Pt 24): p. 4809-18. 
27. Hartmann, C. and C.J. Tabin, Wnt-14 plays a pivotal role in inducing synovial joint formation in the developing appendicular skeleton. Cell, 2001. 104(3): p. 341-51.

28. Liu, S., et al., Overexpression of Wnt11 promotes chondrogenic differentiation of bone marrow-derived mesenchymal stem cells in synergism with TGF-beta. Mol Cell Biochem, 2014. 390(1-2): p. 123-31.

29. Dell'accio, F., et al., Identification of the molecular response of articular cartilage to injury, by microarray screening: Wnt-16 expression and signaling after injury and in osteoarthritis. Arthritis Rheum, 2008. 58(5): p. 1410-21.

30. Bradley, E.W. and M.H. Drissi, WNT5A regulates chondrocyte differentiation through differential use of the CaN/NFAT and IKK/NF-kappaB pathways. Mol Endocrinol, 2010. 24(8): p. 1581-93.

31. van der Kraan, P.M., E.N. Blaney Davidson, and W.B. van den Berg, Bone morphogenetic proteins and articular cartilage: To serve and protect or a wolf in sheep clothing's? Osteoarthritis Cartilage, 2010. 18(6): p. 735-41.

32. Im, G.I., J.M. Lee, and H.J. Kim, Wnt inhibitors enhance chondrogenesis of human mesenchymal stem cells in a long-term pellet culture. Biotechnol Lett, 2011. 33(5): p. 1061-8.

33. Pasold, J., et al., Reduced expression of Sfrp1 during chondrogenesis and in articular chondrocytes correlates with osteoarthritis in STR/ort mice. Exp Cell Res, 2013. 319(5): p. 649-59.

34. Chan, B.Y. and C.B. Little, The interaction of canonical bone morphogenetic proteinand Wnt-signaling pathways may play an important role in regulating cartilage degradation in osteoarthritis. Arthritis Res Ther, 2012. 14(3): p. 119.

35. Miyazawa, K., et al., Two major Smad pathways in TGF-beta superfamily signalling. Genes Cells, 2002. 7(12): p. 1191-204.

36. Chen, D., M. Zhao, and G.R. Mundy, Bone morphogenetic proteins. Growth Factors, 2004. 22(4): p. 233-41.

37. Steinert, A.F., et al., Hypertrophy is induced during the in vitro chondrogenic differentiation of human mesenchymal stem cells by bone morphogenetic protein-2 and bone morphogenetic protein-4 gene transfer. Arthritis Res Ther, 2009. 11(5): p. R148.

38. Shu, B., et al., BMP2, but not BMP4, is crucial for chondrocyte proliferation and maturation during endochondral bone development. J Cell Sci, 2011. 124(Pt 20): p. 3428-40. 
39. Zhang, M., et al., BMP-2 modulates beta-catenin signaling through stimulation of Lrp5 expression and inhibition of beta-TrCP expression in osteoblasts. J Cell Biochem, 2009. 108(4): p. 896-905.

40. Retting, K.N., et al., BMP canonical Smad signaling through Smad1 and Smad5 is required for endochondral bone formation. Development, 2009. 136(7): p. 1093-104.

41. Miyazono, K., S. Maeda, and T. Imamura, Coordinate regulation of cell growth and differentiation by TGF-beta superfamily and Runx proteins. Oncogene, 2004. 23(24): p. 4232-7.

42. Leboy, P., et al., Smad-Runx interactions during chondrocyte maturation. J Bone Joint Surg Am, 2001. 83-A Suppl 1(Pt 1): p. S15-22.

43. Yu, Y.Y., et al., Immunolocalization of BMPs, BMP antagonists, receptors, and effectors during fracture repair. Bone, 2010. 46(3): p. 841-51.

44. Caron, M.M., et al., Hypertrophic differentiation during chondrogenic differentiation of progenitor cells is stimulated by BMP-2 but suppressed by BMP-7. Osteoarthritis Cartilage, 2013. 21(4): p. 604-13.

45. Tsumaki, N., et al., Bone morphogenetic protein signals are required for cartilage formation and differently regulate joint development during skeletogenesis. J Bone Miner Res, 2002. 17(5): p. 898-906.

46. Zou, L., et al., Molecular mechanism of osteochondroprogenitor fate determination during bone formation. Adv Exp Med Biol, 2006. 585: p. 431-41.

47. Karl, A., et al., Thyroid hormone-induced hypertrophy in mesenchymal stem cell chondrogenesis is mediated by bone morphogenetic protein-4. Tissue Eng Part A, 2014. 20(1-2): p. 178-88.

48. Barry, F., et al., Chondrogenic differentiation of mesenchymal stem cells from bone marrow: differentiation-dependent gene expression of matrix components. Exp Cell Res, 2001. 268(2): p. 189-200.

49. Weiss, S., et al., Impact of growth factors and PTHrP on early and late chondrogenic differentiation of human mesenchymal stem cells. J Cell Physiol, 2010. 223(1): p. 8493.

50. Song, J.J., et al., Connective tissue growth factor (CTGF) acts as a downstream mediator of TGF-betal to induce mesenchymal cell condensation. J Cell Physiol, 2007. 210(2): p. 398-410. 
51. Tuli, R., et al., Transforming growth factor-beta-mediated chondrogenesis of human mesenchymal progenitor cells involves $N$-cadherin and mitogen-activated protein kinase and Wnt signaling cross-talk. J Biol Chem, 2003. 278(42): p. 41227-36.

52. Ballock, R.T., et al., TGF-beta 1 prevents hypertrophy of epiphyseal chondrocytes: regulation of gene expression for cartilage matrix proteins and metalloproteases. Dev Biol, 1993. 158(2): p. 414-29.

53. Tschan, T., et al., Autocrine or paracrine transforming growth factor-beta modulates the phenotype of chick embryo sternal chondrocytes in serum-free agarose culture. $\mathrm{J}$ Biol Chem, 1993. 268(7): p. 5156-61.

54. Dieudonne, S.C., et al., Opposite effects of osteogenic protein and transforming growth factor beta on chondrogenesis in cultured long bone rudiments. J Bone Miner Res, 1994. 9(6): p. 771-80.

55. Bohme, K., K.H. Winterhalter, and P. Bruckner, Terminal differentiation of chondrocytes in culture is a spontaneous process and is arrested by transforming growth factor-beta 2 and basic fibroblast growth factor in synergy. Exp Cell Res, 1995. 216(1): p. 191-8.

56. Hellingman, C.A., et al., Smad signaling determines chondrogenic differentiation of bone-marrow-derived mesenchymal stem cells: inhibition of Smad1/5/8P prevents terminal differentiation and calcification. Tissue Eng Part A, 2011. 17(7-8): p. 115767.

57. Shen, J., et al., Deletion of the transforming growth factor beta receptor type II gene in articular chondrocytes leads to a progressive osteoarthritis-like phenotype in mice. Arthritis Rheum, 2013. 65(12): p. 3107-19.

58. Yang, X., et al., TGF-beta/Smad3 signals repress chondrocyte hypertrophic differentiation and are required for maintaining articular cartilage. J Cell Biol, 2001. 153(1): p. 35-46.

59. Wang, W., D. Rigueur, and K.M. Lyons, TGFbeta signaling in cartilage development and maintenance. Birth Defects Res C Embryo Today, 2014. 102(1): p. 37-51.

60. Zuscik, M.J., et al., 5-azacytidine alters TGF-beta and BMP signaling and induces maturation in articular chondrocytes. J Cell Biochem, 2004. 92(2): p. 316-31.

61. Li, T.F., et al., Smad3-deficient chondrocytes have enhanced BMP signaling and accelerated differentiation. J Bone Miner Res, 2006. 21(1): p. 4-16.

62. Furumatsu, T., et al., Smad3 induces chondrogenesis through the activation of SOX9 via CREB-binding protein/p300 recruitment. J Biol Chem, 2005. 280(9): p. 8343-50. 
63. Kang, J.S., et al., Repression of Runx2 function by TGF-beta through recruitment of class II histone deacetylases by Smad3. Embo j, 2005. 24(14): p. 2543-55.

64. Narcisi, R., et al., TGF beta-1 administration during ex vivo expansion of human articular chondrocytes in a serum-free medium redirects the cell phenotype toward hypertrophy. J Cell Physiol, 2012. 227(9): p. 3282-90.

65. Shen, J., S. Li, and D. Chen, TGF-beta signaling and the development of osteoarthritis. Bone Res, 2014. 2.

66. van der Kraan, P.M., et al., TGF-beta signaling in chondrocyte terminal differentiation and osteoarthritis: modulation and integration of signaling pathways through receptor-Smads. Osteoarthritis Cartilage, 2009. 17(12): p. 1539-45.

67. Yang, Z., et al., Temporal activation of beta-catenin signaling in the chondrogenic process of mesenchymal stem cells affects the phenotype of the cartilage generated. Stem Cells Dev, 2012. 21(11): p. 1966-76.

68. Itasaki, N. and S. Hoppler, Crosstalk between Wnt and bone morphogenic protein signaling: a turbulent relationship. Dev Dyn, 2010. 239(1): p. 16-33.

69. Papathanasiou, I., K.N. Malizos, and A. Tsezou, Bone morphogenetic protein-2induced Wnt/beta-catenin signaling pathway activation through enhanced lowdensity-lipoprotein receptor-related protein 5 catabolic activity contributes to hypertrophy in osteoarthritic chondrocytes. Arthritis Res Ther, 2012. 14(2): p. R82.

70. Provot, S., et al., Nkx3.2/Bapxl acts as a negative regulator of chondrocyte maturation. Development, 2006. 133(4): p. 651-62.

71. Zhang, M., et al., PTHrP prevents chondrocyte premature hypertrophy by inducing cyclin-D1-dependent Runx2 and Runx3 phosphorylation, ubiquitylation and proteasomal degradation. J Cell Sci, 2009. 122(Pt 9): p. 1382-9.

72. Huang, W., et al., Phosphorylation of SOX9 by cyclic AMP-dependent protein kinase A enhances SOX9's ability to transactivate a Col2al chondrocyte-specific enhancer. Mol Cell Biol, 2000. 20(11): p. 4149-58.

73. Worthley, D.L., et al., Gremlin 1 identifies a skeletal stem cell with bone, cartilage, and reticular stromal potential. Cell, 2015. 160(1-2): p. 269-84.

74. Kim, E.J., et al., Ihh and Runx2/Runx3 signaling interact to coordinate early chondrogenesis: a mouse model. PLoS One, 2013. 8(2): p. e55296.

75. Amano, K., et al., Indian hedgehog signaling regulates transcription and expression of collagen type X via Runx2/Smads interactions. J Biol Chem, 2014. 289(36): p. 24898-910. 
76. Vortkamp, A., et al., Regulation of rate of cartilage differentiation by Indian hedgehog and PTH-related protein. Science, 1996. 273(5275): p. 613-22.

77. Karp, S.J., et al., Indian hedgehog coordinates endochondral bone growth and morphogenesis via parathyroid hormone related-protein-dependent and-independent pathways. Development, 2000. 127(3): p. 543-8.

78. Kawashima-Ohya, Y., et al., Effects of parathyroid hormone (PTH) and PTH-related peptide on expressions of matrix metalloproteinase-2, -3 , and -9 in growth plate chondrocyte cultures. Endocrinology, 1998. 139(4): p. 2120-7.

79. Lanske, B., et al., Ablation of the PTHrP gene or the PTH/PTHrP receptor gene leads to distinct abnormalities in bone development. J Clin Invest, 1999. 104(4): p. 399-407.

80. Mak, K.K., et al., Indian hedgehog signals independently of PTHrP to promote chondrocyte hypertrophy. Development, 2008. 135(11): p. 1947-56.

81. Amizuka, N., et al., Programmed cell death of chondrocytes and aberrant chondrogenesis in mice homozygous for parathyroid hormone-related peptide gene deletion. Endocrinology, 1996. 137(11): p. 5055-67.

82. Weir, E.C., et al., Targeted overexpression of parathyroid hormone-related peptide in chondrocytes causes chondrodysplasia and delayed endochondral bone formation. Proc Natl Acad Sci U S A, 1996. 93(19): p. 10240-5.

83. Jiang, J., et al., Interaction between zonal populations of articular chondrocytes suppresses chondrocyte mineralization and this process is mediated by PTHrP. Osteoarthritis Cartilage, 2008. 16(1): p. 70-82.

84. Fischer, J., et al., Human articular chondrocytes secrete parathyroid hormone-related protein and inhibit hypertrophy of mesenchymal stem cells in coculture during chondrogenesis. Arthritis Rheum, 2010. 62(9): p. 2696-706.

85. Fischer, J., et al., Intermittent PTHrP(1-34) Exposure Augments Chondrogenesis and Reduces Hypertrophy of Mesenchymal Stromal Cells. Stem Cells Dev, 2014.

86. Mueller, M.B., et al., Effect of parathyroid hormone-related protein in an in vitro hypertrophy model for mesenchymal stem cell chondrogenesis. Int Orthop, 2013. 37(5): p. 945-51.

87. Kim, Y.J., H.J. Kim, and G.I. Im, PTHrP promotes chondrogenesis and suppresses hypertrophy from both bone marrow-derived and adipose tissue-derived MSCs. Biochem Biophys Res Commun, 2008. 373(1): p. 104-8.

88. Kafienah, W., et al., Three-dimensional cartilage tissue engineering using adult stem cells from osteoarthritis patients. Arthritis Rheum, 2007. 56(1): p. 177-87. 
89. Lanske, B., et al., PTH/PTHrP receptor in early development and Indian hedgehogregulated bone growth. Science, 1996. 273(5275): p. 663-6.

90. Lee, J.M. and G.I. Im, PTHrP isoforms have differing effect on chondrogenic differentiation and hypertrophy of mesenchymal stem cells. Biochem Biophys Res Commun, 2012. 421(4): p. 819-24.

91. Bian, L., et al., Enhanced MSC chondrogenesis following delivery of TGF-beta3 from alginate microspheres within hyaluronic acid hydrogels in vitro and in vivo. Biomaterials, 2011. 32(27): p. 6425-34.

92. Guo, X., et al., The Wnt/beta-catenin pathway interacts differentially with PTHrP signaling to control chondrocyte hypertrophy and final maturation. PLoS One, 2009. 4(6): p. e6067.

93. Ellman, M.B., et al., Fibroblast growth factor control of cartilage homeostasis. J Cell Biochem, 2013. 114(4): p. 735-42.

94. Davidson, D., et al., Fibroblast growth factor $(F G F) 18$ signals through FGF receptor 3 to promote chondrogenesis. J Biol Chem, 2005. 280(21): p. 20509-15.

95. Chia, S.L., et al., Fibroblast growth factor 2 is an intrinsic chondroprotective agent that suppresses ADAMTS-5 and delays cartilage degradation in murine osteoarthritis. Arthritis Rheum, 2009. 60(7): p. 2019-27.

96. Hung, I.H., et al., FGF9 regulates early hypertrophic chondrocyte differentiation and skeletal vascularization in the developing stylopod. Dev Biol, 2007. 307(2): p. 300-13.

97. Lewandoski, M., X. Sun, and G.R. Martin, Fgf8 signalling from the AER is essential for normal limb development. Nat Genet, 2000. 26(4): p. 460-3.

98. Montero, A., et al., Disruption of the fibroblast growth factor-2 gene results in decreased bone mass and bone formation. J Clin Invest, 2000. 105(8): p. 1085-93.

99. Yan, D., D. Chen, and H.J. Im, Fibroblast growth factor-2 promotes catabolism via FGFR1-Ras-Raf-MEK1/2-ERK1/2 axis that coordinates with the PKCdelta pathway in human articular chondrocytes. J Cell Biochem, 2012. 113(9): p. 2856-65.

100. Uchii, M., et al., Role of fibroblast growth factor 8 (FGF8) in animal models of osteoarthritis. Arthritis Res Ther, 2008. 10(4): p. R90.

101. Liu, Z., et al., FGF18 is required for early chondrocyte proliferation, hypertrophy and vascular invasion of the growth plate. Dev Biol, 2007. 302(1): p. 80-91.

102. Narayana, J. and W.A. Horton, FGFR3 biology and skeletal disease. Connect Tissue Res, 2015: p. 1-7. 
103. Shung, C.Y., et al., Disruption of a Sox9-beta-catenin circuit by mutant Fgfr3 in thanatophoric dysplasia type II. Hum Mol Genet, 2012. 21(21): p. 4628-44.

104. Maruyama, T., et al., The balance of WNT and FGF signaling influences mesenchymal stem cell fate during skeletal development. Sci Signal, 2010. 3(123): p. ra40.

105. Minina, E., et al., Interaction of FGF, Ihh/Pthlh, and BMP signaling integrates chondrocyte proliferation and hypertrophic differentiation. Dev Cell, 2002. 3(3): p. 439-49.

106. Yoon, B.S., et al., BMPs regulate multiple aspects of growth-plate chondrogenesis through opposing actions on FGF pathways. Development, 2006. 133(23): p. 466778.

107. Colvin, J.S., et al., Skeletal overgrowth and deafness in mice lacking fibroblast growth factor receptor 3. Nat Genet, 1996. 12(4): p. 390-7.

108. Parker, E.A., et al., Spatial and temporal regulation of GH-IGF-related gene expression in growth plate cartilage. J Endocrinol, 2007. 194(1): p. 31-40.

109. Hunziker, E.B., J. Wagner, and J. Zapf, Differential effects of insulin-like growth factor I and growth hormone on developmental stages of rat growth plate chondrocytes in vivo. J Clin Invest, 1994. 93(3): p. 1078-86.

110. Middleton, J., A. Manthey, and J. Tyler, Insulin-like growth factor (IGF) receptor, IGF-I, interleukin-1 beta (IL-1 beta), and IL-6 mRNA expression in osteoarthritic and normal human cartilage. J Histochem Cytochem, 1996. 44(2): p. 133-41.

111. Abbaspour, A., et al., Continuous infusion of insulin-like growth factor-I into the epiphysis of the tibia. Int Orthop, 2008. 32(3): p. 395-402.

112. Longobardi, L., et al., Subcellular localization of IRS-1 in IGF-I-mediated chondrogenic proliferation, differentiation and hypertrophy of bone marrow mesenchymal stem cells. Growth Factors, 2009. 27(5): p. 309-20.

113. Cucchiarini, M. and H. Madry, Overexpression of human IGF-I via direct rAAVmediated gene transfer improves the early repair of articular cartilage defects in vivo. Gene Ther, 2014. 21(9): p. 811-9.

114. Wang, J., J. Zhou, and C.A. Bondy, Igf1 promotes longitudinal bone growth by insulin-like actions augmenting chondrocyte hypertrophy. Faseb j, 1999. 13(14): p. 1985-90.

115. Repudi, S.R., M. Patra, and M. Sen, WISP3-IGF1 interaction regulates chondrocyte hypertrophy. J Cell Sci, 2013. 126(Pt 7): p. 1650-8. 
116. Mushtaq, T., et al., Insulin-like growth factor-I augments chondrocyte hypertrophy and reverses glucocorticoid-mediated growth retardation in fetal mice metatarsal cultures. Endocrinology, 2004. 145(5): p. 2478-86.

117. Bohme, K., et al., Induction of proliferation or hypertrophy of chondrocytes in serumfree culture: the role of insulin-like growth factor-I, insulin, or thyroxine. J Cell Biol, 1992. 116(4): p. 1035-42.

118. Wang, L., Y.Y. Shao, and R.T. Ballock, Thyroid hormone-mediated growth and differentiation of growth plate chondrocytes involves IGF-1 modulation of betacatenin signaling. J Bone Miner Res, 2010. 25(5): p. 1138-46.

119. Wang, Y., et al., Insulin-like growth factor-I is essential for embryonic bone development. Endocrinology, 2006. 147(10): p. 4753-61.

120. Silver, I.A., Measurement of $\mathrm{pH}$ and ionic composition of pericellular sites. Philos Trans R Soc Lond B Biol Sci, 1975. 271(912): p. 261-72.

121. Leijten, J., et al., Metabolic programming of mesenchymal stromal cells by oxygen tension directs chondrogenic cell fate. Proc Natl Acad Sci U S A, 2014. 111(38): p. 13954-9.

122. Lee, H.H., et al., Hypoxia enhances chondrogenesis and prevents terminal differentiation through PI3K/Akt/FoxO dependent anti-apoptotic effect. Sci Rep, 2013. 3: p. 2683.

123. Mariani, E., L. Pulsatelli, and A. Facchini, Signaling pathways in cartilage repair. Int J Mol Sci, 2014. 15(5): p. 8667-98.

124. Zhou, N., et al., HIF-lalpha as a Regulator of BMP2-Induced Chondrogenic Differentiation, Osteogenic Differentiation, and Endochondral Ossification in Stem Cells. Cell Physiol Biochem, 2015. 36(1): p. 44-60.

125. Saito, T., et al., Transcriptional regulation of endochondral ossification by HIF2alpha during skeletal growth and osteoarthritis development. Nat Med, 2010. 16(6): p. 678-86.

126. Yang, S., et al., Hypoxia-inducible factor-2alpha is a catabolic regulator of osteoarthritic cartilage destruction. Nat Med, 2010. 16(6): p. 687-93.

127. $\mathrm{Wu}, \mathrm{L}$. , et al., Insights on biology and pathology of HIF-1alpha/-2alpha, TGFbeta/BMP, Wnt/beta-catenin, and NF-kappaB pathways in osteoarthritis. Curr Pharm Des, 2012. 18(22): p. 3293-312. 
128. Tamiya, H., et al., Analysis of the Runx2 promoter in osseous and non-osseous cells and identification of HIF2A as a potent transcription activator. Gene, 2008. 416(1-2): p. 53-60.

129. Yang, S., et al., NAMPT (visfatin), a direct target of hypoxia-inducible factor-2alpha, is an essential catabolic regulator of osteoarthritis. Ann Rheum Dis, 2015. 74(3): p. 595-602.

130. Ryu, J.H., et al., Hypoxia-inducible factor-2alpha regulates Fas-mediated chondrocyte apoptosis during osteoarthritic cartilage destruction. Cell Death Differ, 2012. 19(3): p. 440-50.

131. Lafont, J.E., S. Talma, and C.L. Murphy, Hypoxia-inducible factor 2alpha is essential for hypoxic induction of the human articular chondrocyte phenotype. Arthritis Rheum, 2007. 56(10): p. 3297-306. 


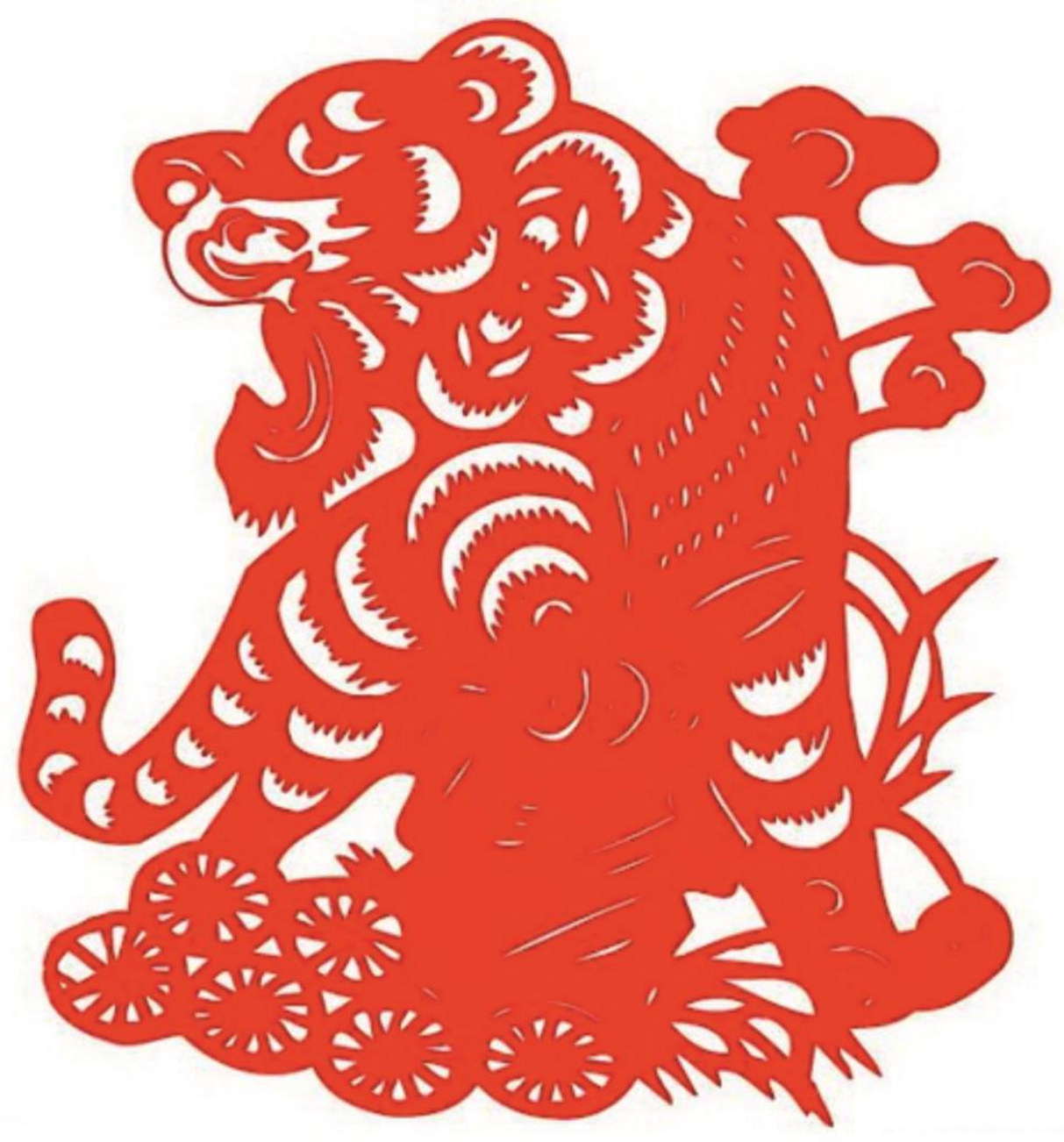




\section{Chapter 3}

\section{The expression of DKK1 and FRZB are negatively correlated to local inflammation and OA severity}

Xiaobin Huang ${ }^{1}$, Leilei Zhong ${ }^{1}$, Eefje van Helvoort ${ }^{2}$, Floris Lafeber ${ }^{2}$, Simon Mastbergen $^{2}$, Jan Hendriks ${ }^{1}$, Janine N. Post ${ }^{1}$, Marcel Karperien ${ }^{1}$

${ }^{1}$ Department of Developmental BioEngineering, MIRA Institute for Biomedical Technology and Technical Medicine, University of Twente, Enschede, the Netherlands

${ }^{2}$ Department of Rheumatology \& Clinical Immunology, Regenerative Medicine Center Utrecht, University Medical Center Utrecht, University Utrecht, Utrecht, the Netherlands.

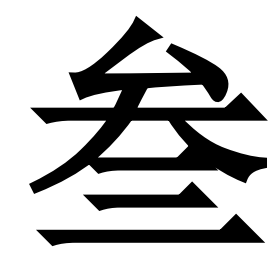

天地不仁，以万物为刍狗; 圣人不仁，以百姓为刍狗。

Heaven and earth are ruthless; to them the Ten Thousand Things are but as straw dogs.

The Sage is ruthless; to him the people are but as straw dogs. 


\section{Abstract}

Purpose: To investigate the presence of WNT antagonists Dickkopf-related protein 1 (DKK1), Frizzled-related protein (FRZB) and Gremlin 1 (GREM1) in synovial fluid (SF) and serum respectively from end-stage osteoarthritis $(\mathrm{OA})$ patients, and correlate their expression with other markers of OA.

Methods: In a cross-sectional study, SF and serum were collected from OA patients $(\mathrm{n}=132)$ with or without treatment by celecoxib and naproxen shortly before joint replacement surgery. The concentrations (conc.) of DKK1, FRZB and GREM1 in SF and serum were determined using immunoassays. Odds ratios (ORs) and 95\% confidence intervals (95\% CIs) were calculated using logistic regression analyses with adjustment for potential covariates. Correlation measurements were performed with previously assessed disease markers.

Results: The OA patients with the celecoxib treatment till surgery have higher median SF FRZB values compared to the control (no treatment); the celecoxib 3-days before surgery stopped treatment group has higher median serum FRZB values than the control and the naproxen treatment group. The combinational analysis of SF DKK1 and SF FRZB negatively correlated with macroscopic scores of cartilage $(\mathrm{R}=-0.217, \mathrm{P}=0.014)$ and with histological synovium scores $(\mathrm{R}=-0.202, \mathrm{P}=0.031)$ in $\mathrm{OA}$ patients. The expression of $\mathrm{DKK} 1$ and FRZB showed the same trend in SF and in serum. Furthermore, the SF concentration of DKK1 was positively correlated with FRZB in both $\mathrm{SF}(\mathrm{R}=0.32, \mathrm{P}<0.001)$ and $\operatorname{serum}(\mathrm{R}=0.283, \mathrm{P}=0.001)$. In contrast, it was negatively correlated with Nitric Oxide (NO)/weight synovium $(\mathrm{R}=-0.327$, $\mathrm{P}=0.001)$ and $\mathrm{IL} 1 \beta /$ weight synovium $(\mathrm{R}=-0.186, \mathrm{P}=0.059)$. Both $\mathrm{SF} \mathrm{DKK} 1(\mathrm{R}=0.194, \mathrm{P}=0.045)$ and serum DKK1 $(\mathrm{R}=0.206, \mathrm{P}=0.034)$ were positively correlated with Western Ontario and McMaster Universities Arthritis Index (WOMAC) total scores. SF FRZB was negatively correlated with $\mathrm{PGE} 2 /$ weight cartilage $(\mathrm{R}=-0.184, \mathrm{P}=0.041)$, IL1 $\beta /$ weight synovium $(\mathrm{R}=-0.164$, $\mathrm{P}=0.096), \mathrm{PGE} 2 /$ weight synovium $(\mathrm{R}=-0.175, \mathrm{P}=0.064), \mathrm{NO} /$ weight synovium $(\mathrm{R}=-0.189$, $\mathrm{P}=0.047)$ and age $(\mathrm{R}=-0.220, \mathrm{P}=0.011)$. However, SF GREM1 was positively correlated with 
IL1 $\beta$ / weight synovium and TNF $\alpha$ /weight synovium. Serum GREM1 positively correlated with PGE2/weight cartilage, TNFa/weight synovium, TNF synovium, weight and BMI, while it was negatively correlated with $\mathrm{NO} /$ weight cartilage.

Conclusions: Our findings suggest that DKK1 and FRZB are involved in OA progression. Celecoxib treatment till and until 3 days before surgery stimulate FRZB secretion and serum FRZB presence in SF and serum. DKK1 and FRZB were negatively correlated with OA severity and multiple pro-inflammatory cytokines. Our data indicate that DKK1 and FRZB can be joint-specific biomarkers and synovial fluid might be more sensitive than serum to reflect changes in local disease such as OA.

Keywords: Dickkopf-related protein 1 (DKK1); Frizzled-related protein (FRZB); Gremlin 1 (GREM1); synovial fluid; osteoarthritis (OA); pro-inflammatory cytokines; ELISA. 


\section{Introduction}

Osteoarthritis (OA) is the most common chronic joint disorder, characterized by progressive degradation of joint cartilage, formation of bony tissue at the joint margins (osteophytes), subchondral bone changes (sclerosis), inflammation of the synovial membrane, and impairment of the periarticular soft tissues [1-4]. Although large research effort has been devoted in recent years to explore the molecular mechanism underlying this disease, the pathogenesis of OA is still largely unknown and no effective treatment is available.

Multiple signal pathways have been implicated in the pathogenesis of OA [5-8], such as the alteration of secreted wingless-type MMTV integration site (WNT) and the bone morphogenetic protein (BMP) signal pathways $[9,10]$. In articular cartilage, moderate activity of WNT is essential for chondrocyte proliferation and cell phenotype [11], while overexpression can lead to cartilage disease. It has been shown that $\beta$-catenin, the key signaling protein in the WNT pathway is highly expressed in severe OA compared with preserved OA, and that activation of $\beta$-catenin in articular cartilage leads to OA-like phenotype in in vivo studies $[12,13]$. In addition, dysregulation of various WNT proteins and their receptors have been documented in human joint disorders [14]. BMPs are widely known as potent inducers of bone. In vivo injection of BMP2 leads to osteophyte formation in mouse knee joints [15] and increased expression of BMP2 has been reported in OA chondrocytes and cartilage [16, 17]. Moreover, BMP2-induced WNT/ $\beta$-catenin signaling through LRP-5 may contribute to chondrocyte hypertrophy and cartilage degradation in OA [16]. In contrast to BMP2, 4 and 6 which may promote chondrocyte hypertrophy, BMP7 could inhibit this process, although the exact mechanism is still unknown $[18,19]$.

WNT and BMP activators and inhibitors could therefore play an important role in OA pathogenesis. Multiple whole genome studies have shown that the WNT antagonist FRZB is a candidate gene associated with hip OA in females [20]. This association of FRZB with susceptibility for OA is further confirmed in other cohorts [21-24]. In addition, it was shown 
that there is a tendency that higher FRZB levels in serum are related with a modest reduction in risk of incident RHOA [25]. DKK1, another WNT antagonist, was associated with reduced progression of radiographic hip osteoarthritis (RHOA) in elderly women [25]. This is in line with our previous study showing that DKK1 and FRZB have protective roles in cartilage by prevent hypertrophic differentiation [26] and the loss of their expression is observed in OA [27]. Considering the role of the BMP pathway, it has been shown that upregulation of GREM1, which is a BMPs antagonist, is observed in human OA chondrocytes [28] and its expression increases with OA progression in dog OA [29]. This suggests an involvement of GREM1 in OA pathophysiology.

Even though OA is not widely seen as an inflammatory disease like rheumatic arthritis, serological and histological evidence of synovitis is commonly found in OA. In fact, synovial inflammation can be involved in the early onset of OA as well as in advanced stages of OA [30, 31]. Interleukin-1 $\beta$ (IL-1 $\beta$ ) and tumor necrosis factor- $\alpha$ (TNF- $\alpha$ ) play a key role in the destructive process, by stimulating the expression and release of proteases such as matrix metalloproteinases (MMPs), aggrecanases (ADAMTS) which degrade collagen and aggrecan. These proinflammatory cytokines stimulate synthesis and release of nitric oxide (NO) and prostaglandin-E2 (PGE2), which are produced in elevated amounts during the degenerative process, contributing to joint pathology [30, 32].

It has been reported that anabolic or catabolic molecules are released from joint tissue enter into the synovial fluid and serum during joint disease [33-35]. This indicates that molecular changes in OA can be reflected by altered serum and synovial fluid levels of these factors.

In this study, a comprehensive cross-sectional analysis was performed in synovial fluid and serum samples collected from OA patients. We measured the concentration of DKK1, FRZB and GREM1 in patients with or without pharmaceutical treatment in synovial fluid and serum and correlated their expression levels with multiple OA features (macroscopically, histologically, and biochemically) and inflammatory factors. 


\section{Materials and methods}

\section{Subjects}

Material of one hundred and thirty-two patients with severe knee osteoarthritis, who were eligible for total knee replacement surgery, and were treated at the Sint Franciscus Gasthuis Rotterdam, the Netherlands, could be obtained. Exclusion criteria were a total knee replacement for other reasons than OA, a history of gastrointestinal bleedings or perforation, and an increased risk for cardiovascular diseases (patients with a history of cardiovascular disease, with untreated hypertension, with angina pectoris, and patients on oral anticoagulants). These samples have been collected in previous investigations and the full data set was available for this study [36]. The study was conducted according to the declaration of Helsinki and received ethical approval. Each patient gave written informed consent before participating in the study.

Originally patients were randomized to one of four treatment groups 4 weeks prior to total knee replacement surgery. Patients were randomized to celecoxib 2dd200mg (until surgery), celecoxib 2dd200mg until 3 days before surgery (to control for the obligated stop of naproxen treatment 3 days before surgery), and naproxen 3dd250mg until 3 days before surgery, or no treatment (controls). Because of its platelet-inhibiting effect, the use of naproxen had to be stopped 3 days prior to surgery. All 4 groups included 43 patients originally. Because of the increased risk for gastrointestinal adverse effects with the use of naproxen, all patients also received omeprazol 20mg o.d. Complete data sets as obtained in the original study, synovial fluid and serum of 132 patients could be retrieved for this study (Table 1).

\begin{tabular}{lllll}
\hline Main diagnostic groups & & Number & Women(\%) & Age median (range) \\
\hline Total & & 132 & $87(65.9 \%)$ & $66.21(49.67-89.08)$ \\
& No treatment & 32 & $22(68.75 \%)$ & $67.63(56.17-88.17)$ \\
& Celecoxib-full & 34 & $21(61.77 \%)$ & $65.96(52.08-89.08)$ \\
& Celecoxib-3days & 34 & $21(61.77 \%)$ & $65.67(51.67-79.58)$ \\
& Naproxen-3days & 31 & $23(74.19 \%)$ & $65.83(49.67-82.5)$ \\
\hline
\end{tabular}

Table 1 Characteristics of the study subjects. 


\section{Macroscopy and histochemistry}

Macroscopic cartilage damage was evaluated on digital high-resolution photographs of femur surface parts. Severity of cartilage damage was graded from 0-3 (0=fibrillation or focal degeneration, $1=$ degeneration at multiple locations, $2=$ degeneration at multiple locations with focal lesions, and 3=degeneration throughout the tissue with severe focal lesions and focally full cartilage abrasion). Synovial tissue inflammation was evaluated by histochemistry. Briefly, synovial tissue samples were fixed in $4 \%$ phosphate buffered formalin with $2 \%$ sucrose, then stained with Haematoxylin-Eosin. The tissues were sliced and from each sample 3 slices were used for scoring. Synovial tissue inflammation was graded using modified Goldenberg and Cohen scores. Two observers blinded to the source of the samples, scored all samples and the averages of observers were taken as representative score of each donor. In the limited case, when observers scored $>1$ point difference, consensus was sought.

\section{Analysis of DKK1, FRZB and GREM1 in synovial fluid and in serum}

Synovial fluid concentrations of DKK1, FRZB and GREM1 were determined using enzymelinked immunosorbent assay (ELISA) according to the manufacturer guidelines (DKK1, Catalog Number: DY1906, R\&D system; FRZB, Catalog Number: DY192; GREM1, Bioconnect diagnostics).

All samples were diluted 1:2 for DKK1 and FRZB detection, and 1:24 for GREM1 detection according to the sample specificity and manufacture protocols. Randomly selected knee synovial fluid and serum samples were used to assess dilution linearity $(n=3)$, effects of freezethawing $(n=5)$ and spiking recovery $(n=3)$. Details on the technical performance of the DKK1, FRZB and GREM1 ELISA in synovial fluid and serum in terms of dilution linearity and spiking recovery, intra- and inter-assay $\mathrm{CV}$, and the influence of thawing and freezing of samples is presented in Supplementary data Table S1 (for SF) and Table S2 (for serum). 


\section{Analysis of pro-inflammatory cytokines in synovial tissue culture medium}

To assess the IL-1 $\beta$ and TNF $\alpha$ production by the OA cartilage and synovial tissue, samples were cultured individually for 3 days, and subsequently IL-1 $\beta$ and TNF $\alpha$ release in the culture supernatants were determined by ELISA (Biosource) according to manufacturer's instructions and expressed as pg/ml per mg (wet weight) tissue. Prostaglandin-E2 (PGE2) was determined by Enzyme Immuno Assay (EIA, Caymann Chemical) and expressed as pg/ml per mg tissue. NO levels were determined using the standard Griess reaction and expressed as $\mu \mathrm{M}$ per mg (wet weight) of tissue.

\section{Clinical outcome}

Patients were asked to fill out a Western Ontario and McMaster University (WOMAC) questionnaire to evaluate pain, stiffness, and function, before and after medical treatment [37].

\section{Statistics}

For histochemistry, two observers blinded to the source of the samples scored all samples and the average scores of observers was taken as representative score of each sample. In a few cases consensus was sought when observer scored $>1$ point difference. The average of all multiple tissue samples ( $\mathrm{n}=4$ for cartilage histology, $\mathrm{n}=3$ for synovial tissue histology, and $\mathrm{n}=3$ for cytokine production) of each donor was taken as representative value for each donor. Data are subsequently expressed as mean $( \pm \mathrm{SD})$ of donor.

According to Shapiro-Wilk tests synovial fluid and serum DKK1, FRZB and GREM1 concentrations were not normally distributed and therefore non-parametric analyses were conducted. Between-groups comparisons were made using Mann-Whitney rank sum tests, and for correlation analysis Spearman's rank correlation (R) analysis were used. Pearson Chi-square test was used for comparison of genders between subject groups, and for comparison of age Students t-test was used. All tests were 2-tailed, $\mathrm{p}<0.05$ was considered statistically significant, 
$\mathrm{p}$ values $<0.1$ were considered borderline significant. All statistical analyses were performed using SPSS (standard version 15.0, SPSS, Chicago, IL, USA).

\section{Results}

\section{Technical performance of the DKK1, FRZB and GREM1 ELISA in synovial fluid and serum}

In order to assess the accuracy and reliability of the ELISAs, the dilution linearity, spiking recovery, freeze/thaw, intra- and inter repeat assays were performed from 3 to 5 randomly selected synovial fluid and serum samples. LLOD and ULOD for the DKK1, FRZB and GREM1 assays were estimated at $7.77-4000 \mathrm{pg} / \mathrm{ml}, \quad 7.77-8000 \mathrm{pg} / \mathrm{ml}, \quad 57-8000 \mathrm{pg} / \mathrm{ml}$ respectively based on the kit instruction and standard curves ( Supplementary data Table S1 and S2).

To calculate dilution linearity, randomly selected synovial fluid and serum samples $(n=3)$ were prepared in three different dilutions, 1:2, 1:4, 1:8 for DKK1 and FRZB, and 1:24, 1:48, 1:96 for GREM1. Results are expressed as \% recovery: 100 x [(conc. at a specific dilution) divided by (first diluted conc. divided by the times diluted)]. We observed good dilution linearity with mean recoveries between $76 \%$ and $115 \%$ for DKK1, 71\%-120\% for FRZB and $68 \%-131 \%$ for GREM1 respectively, which fall within the acceptance range (Table S1 and S2).

Spiking recovery was calculated from randomly selected synovial fluid and serum samples $(n=3)$ which were spiked using high, middle or low concentrations of standards. Spiking recovery is expressed in \% as recovery: $100 \mathrm{x}$ ((concentration of a sample spiked with a specific amount of standard) divided by (the individual concentration of sample plus the individual concentration from standard)). Good results of spiking recovery were observed (Table S1and S2). 
To analyze freeze-thawing effect on DKK1, FRZB and GREM1 concentrations, randomly selected synovial fluid and serum samples $(n=3)$ were frozen/thawed 3 times. The result showed that the freeze-thaw cycles appeared to have no systematic effect on the measured concentrations of these analytes in synovial fluid and serum (Table S1 and S2).

Intra- and inter assay CV of DKK1, FRZB and GREM1 was calculated using randomly selected synovial fluid and serum samples $(n=3)$ loading 5 repeats (intra) on a single plate or loading duplicates on 4 plates (inter). The results showed that different wells and different plates have no systematic effect on the measured concentrations of these analytes in synovial fluid and serum.

The technical performance results indicate that the DKK1, FRZB and GREM1 values obtained from synovial fluid and serum samples can be determined with confidence. And that there are no factors to depress or elevate the values in the synovial fluid and serum significantly.

\section{The OA patients with celecoxib treatment have higher SF FRZB median value than the control (no treatment)}

The data and material used in this study was originally collected from patients included in randomized clinical trial [36]. The aim was to evaluate in vivo disease modifying activity of short term celecoxib treatment, with the attempt to modulate proteolytic activity (proteoglycan release) as an early indicator for cartilage repair activity, by treating patients in end-stage osteoarthritis with celecoxib shortly before joint replacement surgery followed by a detailed $e x$ vivo assessment of joint tissues with observer blinded evaluation. Analysis of SF FRZB in our study revealed that OA patients with celecoxib treatment have a 2 times higher SF FRZB median value than the control (no treatment) group $(\mathrm{p}=0.009)$; the group treated with celecoxib until 3 days before surgery had a 1.97 times higher Serum FRZB median value than the control $(\mathrm{p}=0.003)$ and 2.13 times higher than the naproxen treatment group (0.034). However, there is no significant difference for expression levels of DKK1 and GREM1 among different treatments (Fig. 1). 

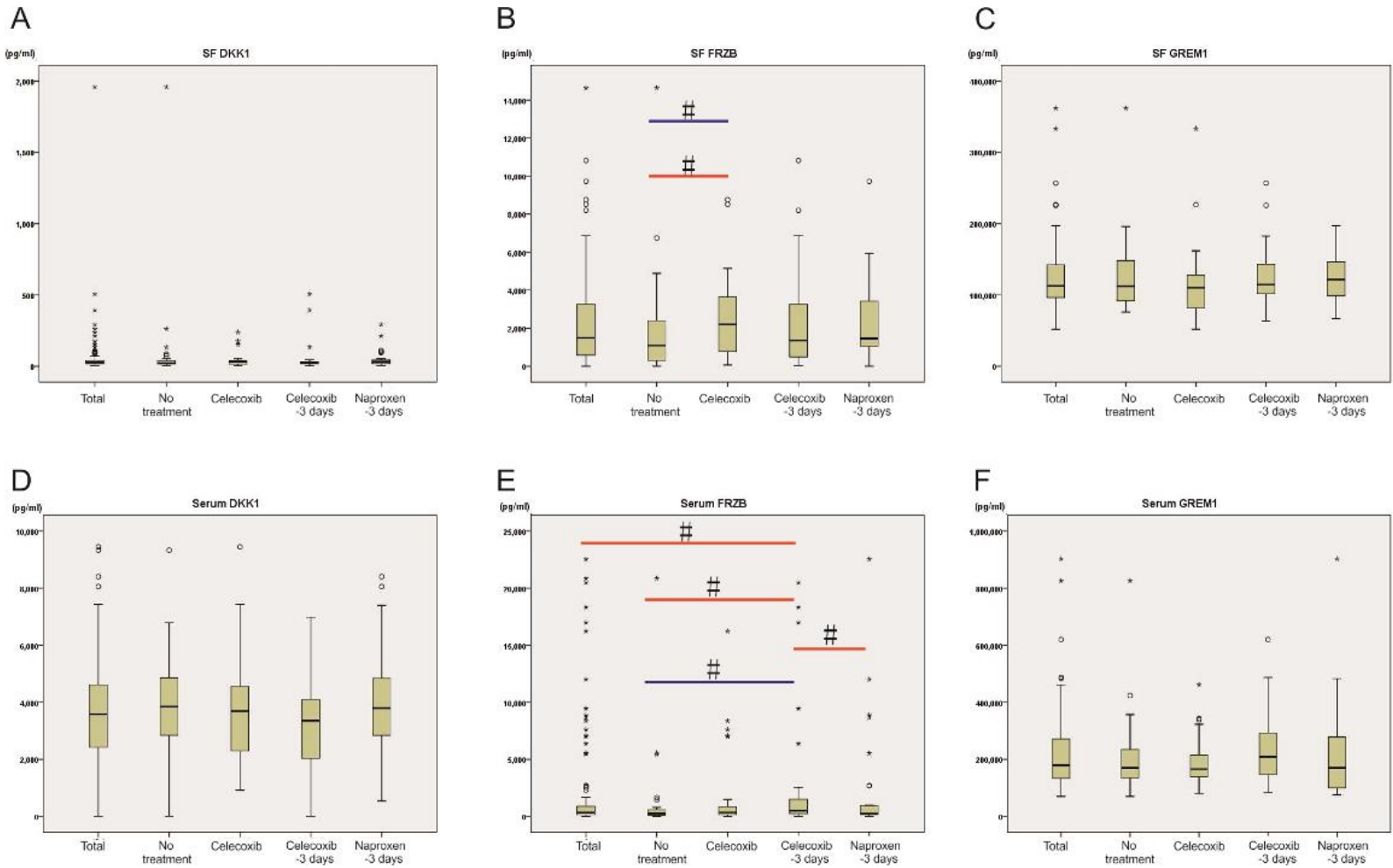

Fig. 1 Concentrations of DKK1, FRZB and GREM1 in SF and serum in no treatment group, Celecoxibfull group (Celecoxib), Celecoxib-3days group and Naproxen-3days group. Samples were grouped according to Table 1 . The results are expressed as median and the 25 th and 75 th percentiles with whiskers (10th and 90th percentiles). Circles and asterisks stand for discrete values and extreme values respectively. The red line means median difference and blue line means distribution difference.

DKK1 and FRZB are expressed in serum and have the same expression trend as their expression in synovial fluid from OA patients

In order to detect changes of the antagonist's expression in SF and serum, their concentrations were measured by ELISA respectively. The DKK1 conc. median (range) is 26.9 (3.9-1958.9) $\mathrm{pg} / \mathrm{ml}$ in $\mathrm{SF}$, much lower than 3589.7 (3.9-9452.5) $\mathrm{pg} / \mathrm{ml}$ in serum; however, the FRZB conc. median (range) is 1486.7 (3.6-14635.8) $\mathrm{pg} / \mathrm{ml}$ in $\mathrm{SF}$, much higher than 332.7 (3.6-22539.8) 
$\mathrm{pg} / \mathrm{ml}$ in serum. The GREM1 conc. median (range) is 112.9 (51.9-362.1) $\mathrm{ng} / \mathrm{ml}$ in SF while 179.89 (70.0-903.4) $\mathrm{ng} / \mathrm{ml}$ in serum. After statistical comparison, we found that both DKK1 and FRZB have a similar expression trend in $\mathrm{SF}$ and serum $(\mathrm{R}=0.305, \mathrm{P}<0.001 ; \mathrm{R}=0.300$, $\mathrm{P}=0.001$ ), However, for GREM1 there was no correlation ( Fig. 2, Table 2).
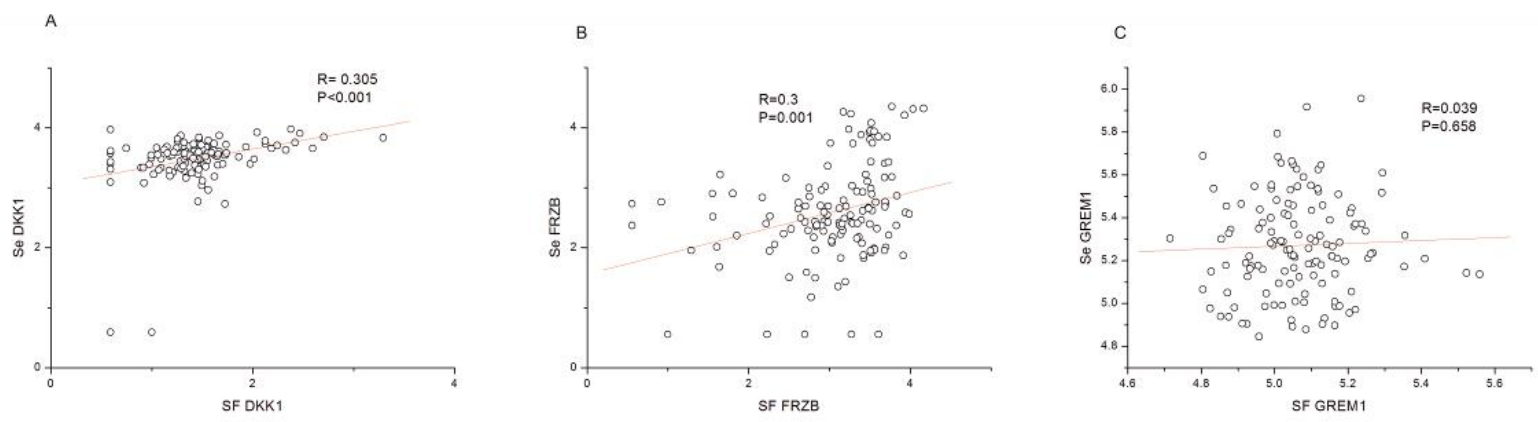

Fig. 2 Correlation of conc. of DKK1 and FRZB between SF and serum. A bivariate scatter plot of DKK1 and FRZB concentrations (showed in denary logarithm) in synovial fluid with the corresponding concentrations in serum $(n=132)$. Spearman coefficient $(R), P$ values and the first order regression lines are indicated.

\begin{tabular}{llllll}
\hline & SF FRZB & SF GREM1 & Se DKK1 R(P) & Se FRZB R(P) & Se GREM1 R(P) \\
\hline SF DKK1 & $\mathbf{0 . 3 2 0}(<\mathbf{0 . 0 0 1})$ & $0.016(0.854)$ & $\mathbf{0 . 3 0 5}(<\mathbf{0 . 0 0 1})$ & $\mathbf{0 . 2 8 3}(\mathbf{0 . 0 0 1})$ & $\mathbf{0 . 1 9 9 ( \mathbf { 0 . 0 2 3 } )}$ \\
SF FRZB & - & $0.030(0.733)$ & $0.043(0.623)$ & $\mathbf{0 . 3 0 0}(\mathbf{0 . 0 0 1})$ & $0.062(0.484)$ \\
SF GREM1 & - & - & $0.016(0.854)$ & $-0.056(0.529)$ & $0.039(0.658)$ \\
& & & & & \\
\hline
\end{tabular}

Table 2. Correlation between DKK1, FRZB, GREM1 in synovial fluid or serum. Spearman coefficient (R) and P values are indicated. Significant correlations are bolded. FRZB, frizzled-related protein; GREM1, Gremlin 1; DKK1, Dickkopf-related protein; SF, synovial fluid; Se, serum.

\section{SF DKK1 and SF FRZB are negatively correlated with OA severity}

In order to assess the severity of cartilage damage after surgery for OA patients, direct macroscopic analysis and histochemistry were performed. Meanwhile, the conc. of DKK1, 
FRZB and GREM1 in the SF and serum from the same patient were measured by ELISA. We found that both SF DKK1 and SF FRZB have a small negative correlation with the macroscopic score $(\mathrm{R}=-0.169, \mathrm{P}=0.056 ; \mathrm{R}=-0.163, \mathrm{P}=0.065)$. Moreover, if $\mathrm{DKK} 1$ and $\mathrm{FRZB}$ log values were combined together, there was a stronger significant negative correlation with the microscopic score $(\mathrm{R}=-0.217, \mathrm{P}=0.014)$ (Fig. 3a). We further assessed the degree of synovial tissue inflammation by Haematoxylin-Eosin staining, modified Goldenberg and Cohen score. It was found that DKK1 in SF significantly negatively correlated with the histologic synovium score $(\mathrm{R}=-0.255, \mathrm{P}=0.006)$ (Fig. 3b). Similarly, if DKK1 and FRZB log values were combined together, there was a significant negative correlation with the histologic synovium score as well $(\mathrm{R}=-0.202$, $\mathrm{P}=0.031)$ (Fig. 3c). GREM1 in serum also slightly negatively correlated with the score $(\mathrm{R}=-0.155, \mathrm{P}=0.099)$ (Table 3).
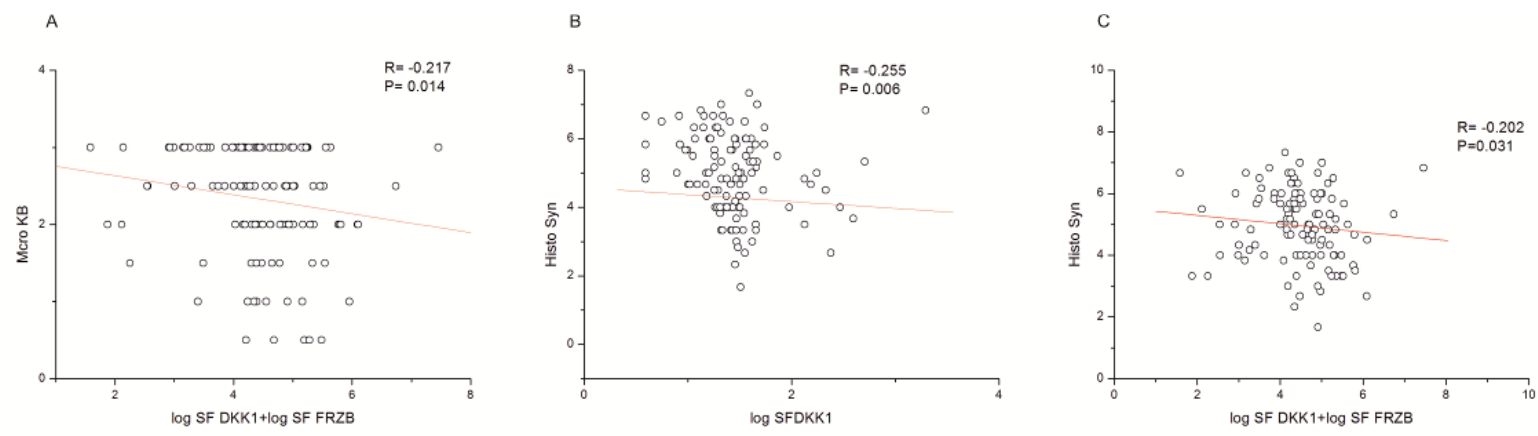

Fig. 3 Correlation between DKK1, FRZB, GREM1 in SF and severity of OA. A bivariate scatter plot of DKK1, FRZB and GREM1 concentrations in synovial fluid $(n=132)$ with the scores of cartilage damage (Marco KB) and synovium inflammation (Histo syn). Spearman coefficient (R), P values and the first order regression lines are indicated.

\begin{tabular}{llllllll}
\hline & SF DKK1 & SF FRZB & $\begin{array}{l}\text { Log SF DKK1 + } \\
\text { Log SF FRZB }\end{array}$ & SF GREM1 & Se DKK1 & Se FRZB & Se GREM1 \\
& & & & & & \\
\hline Macro & $\mathbf{- 0 . 1 7 0}$ & $\mathbf{- 0 . 1 6 3}$ & $\mathbf{- 0 . 2 1 7}(\mathbf{0 . 0 1 4})$ & 0.003 & 0.021 & -0.054 & -0.021 \\
KB & $\mathbf{( 0 . 0 5 5 )}$ & $\mathbf{( 0 . 0 6 5 )}$ & & $(0.976)$ & $(0.820)$ & $(0.551)$ & $(0.812)$ \\
Histo & $\mathbf{- 0 . 2 5 5}$ & -0.114 & $\mathbf{- 0 . 2 0 2}(\mathbf{0 . 0 3 1})$ & -0.075 & $\mathbf{0 . 1 6 3}$ & -0.126 & $\mathbf{- 0 . 1 5 5}$ \\
Syn & $\mathbf{( 0 . 0 0 6 )}$ & $(0.227)$ & & $(0.428)$ & $\mathbf{( 0 . 0 8 3 )}$ & $(0.181)$ & $(\mathbf{0 . 0 9 9})$ \\
\hline
\end{tabular}


Table 3. Correlation between DKK1, FRZB, GREM1 and Macro KB, Histo Syn in synovial fluid or serum. Spearman coefficient (R) and P values are indicated. Significant correlations are bolded. Borderline significant are bolded and italic. FRZB, frizzled-related protein; GREM1, Gremlin 1; DKK1, Dickkopf-related protein; SF, synovial fluid; Se, serum; Macro KB, macrocopic cartilage score; Histo Syn, histology synovium score.

The expression of DKK1 in SF shows positively correlation with FRZB in both SF and serum, and with GREM1 in serum

Since all three factors (DKK1, FRZB and GREM1) are involved in the joint homeostasis, we assessed the correlation between these factor in SF and serum as a next step. The results showed that SF DKK1 positively correlated with FRZB in both $\mathrm{SF}(\mathrm{R}=0.32, \mathrm{P}<0.001)$ (Fig. 4a) and serum ( $\mathrm{R}=0.283, \mathrm{P}=0.001)$ (Fig. 4b). Interestingly, the SF DKK1 conc. was also positively correlated with GREM1 in serum $(\mathrm{R}=0.199, \mathrm{P}=0.023)$ (Fig. 4c, Table 2).
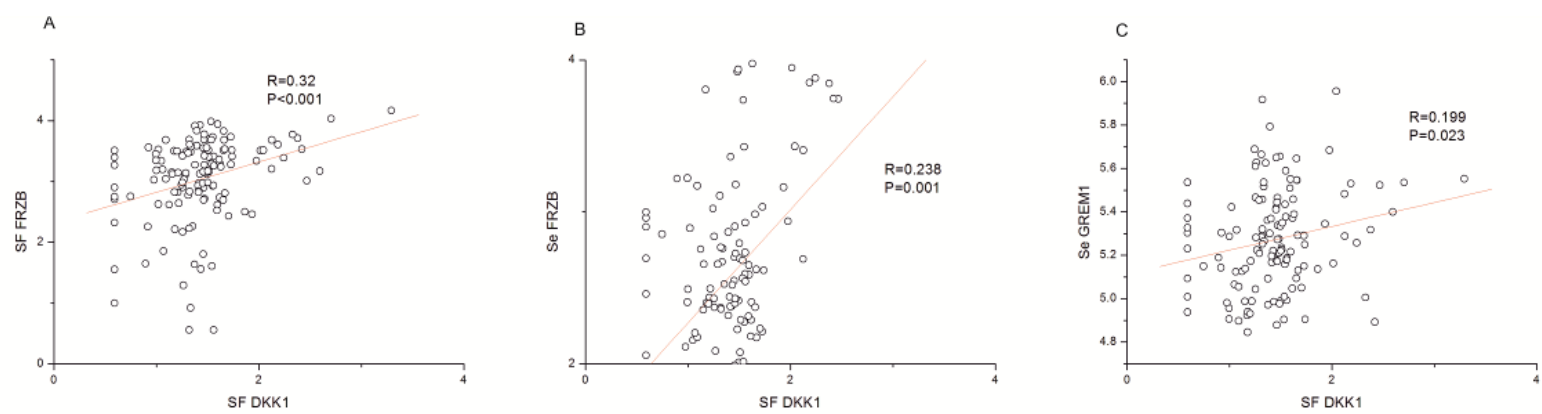

Fig. 4 Correlation between conc. of DKK1, FRZB and GREM1 in SF and serum. A bivariate scatter plot of DKK1 concentrations in synovial fluid with the FRZB conc. both in SF and serum or GREM1 conc. in serum ( $\mathrm{n}=132)$. Spearman coefficient $(\mathrm{R}), \mathrm{P}$ values and the first order regression lines are indicated. 


\section{The expression levels of DKK1 and FRZB, but not of GREM1, are negatively correlated to pro-inflammatory factors}

In order to assess the correlation between the conc. of SF DKK1, FRZB, GREM1 and local inflammation, ELISAs were performed to determine the production of pro-inflammatory factors IL-1 $\beta$ and TNF $\alpha$ by the corresponding OA cartilage and synovial tissue [36]. To gain more information, we additionally measured PGE2 and NO. Age, weight and BMI index were also taken into account. SPSS correlation analysis showed that SF DKK1 negatively correlated with $\mathrm{NO} /$ weight synovium $(\mathrm{R}=-0.327, \mathrm{P}=0.001)$ and $\mathrm{IL1} \beta /$ weight synovium $(\mathrm{R}=-0.186$, $\mathrm{P}=0.059)$. Similarly, SF FRZB negatively correlated with $\mathrm{PGE} 2 /$ weight cartilage $(\mathrm{R}=-0.184$, $\mathrm{P}=0.041)$, IL1 $\beta$ /weight synovium $(\mathrm{R}=-0.164, \mathrm{P}=0.096)$, PGE2/weight synovium $(\mathrm{R}=-0.175$, $\mathrm{P}=0.064), \mathrm{NO} /$ weight synovium $(\mathrm{R}=-0.189, \mathrm{P}=0.047)$ and age $(\mathrm{R}=-0.220, \mathrm{P}=0.011)$. However, SF GREM1 positively correlated with $\operatorname{IL} 1 \beta /$ weight synovium $(\mathrm{R}=0.213, \mathrm{P}=0.03)$ and $\mathrm{TNF} \alpha$ /weight synovium $(\mathrm{R}=0.206, \mathrm{P}=0.041)$. Moreover, serum GREM1 positively correlated with $\mathrm{PGE} 2 /$ weight cartilage $(\mathrm{R}=0.315, \mathrm{P}<0.001), \mathrm{TNF} \alpha$ /weight synovium $(\mathrm{R}=0.274, \mathrm{P}=0.006)$, weight $(\mathrm{R}=0.2, \mathrm{P}=0.023)$ and $\mathrm{BMI}(\mathrm{R}=0.183, \mathrm{P}=0.038)$ while negatively correlated with NO/weight cartilage $(\mathrm{R}=-0.306, \mathrm{P}=0.003)$ (Table 4). WOMAC questionnaire (0-100 scale) was used to collect data on pain, stiffness, and functional ability. Lower score means more pain, stiffness, and functional limitation. Interestingly, Both SF DKK1 $(\mathrm{R}=0.194, \mathrm{P}=0.045)$ and serum DKK1 $(\mathrm{R}=0.206, \mathrm{P}=0.034)$ positively correlated with WOMAC total score (Table 4).

\begin{tabular}{|c|c|c|c|c|c|c|c|}
\hline & $\begin{array}{l}\text { PGE2/weight } \\
\text { (Cartilage), R(P) }\end{array}$ & $\begin{array}{l}\text { NO/weight } \\
\text { (Cartilage), R(P) }\end{array}$ & $\begin{array}{l}\text { IL1B/weight } \\
\text { (synovium), R(P) }\end{array}$ & $\begin{array}{l}\text { TNFa/weight } \\
\text { (synovium), R(P) }\end{array}$ & $\begin{array}{l}\text { PGE2/weight } \\
\text { (synovium), R(P) }\end{array}$ & $\begin{array}{l}\text { NO/weight } \\
\text { (synovium), R(P) }\end{array}$ & $\begin{array}{l}\text { WOMAC total } \\
\text { score }\end{array}$ \\
\hline SF DKK1 & $-0.04(0.656)$ & $-0.327(0.001)$ & $-0.186(0.059)$ & $-0.159(0.115)$ & $-0.149(0.117)$ & $0.092(0.338)$ & $0.194(0.045)$ \\
\hline SF FRZB & $-0.184(0.041)$ & $-0.106(0.314)$ & $-0.164(0.096)$ & $-0.071(0.483)$ & $-0.175(0.064)$ & $-0.189(0.047)$ & $0.101(0.302)$ \\
\hline SF GREM1 & $0.147(0.102)$ & $-0.006(0.954)$ & $0.213(0.03)$ & $0.206(0.041)$ & $0.099(0.298)$ & $0.087(0.366)$ & $-0.085(0.385)$ \\
\hline Se DKK1 & $0.037(0.685)$ & $-0.081(0.442)$ & $0.114(0.251)$ & $0.045(0.662)$ & $0.072(0.451)$ & $-0.037(0.699)$ & $0.206(0.034)$ \\
\hline Se FRZB & $-0.104(0.25)$ & $-0.136(0.195)$ & $-0.233(0.018)$ & $-0.128(0.208)$ & $-0.023(0.810)$ & $-0.098(0.311)$ & $0.037(0.705)$ \\
\hline Se GREM1 & $0.315(<0.001)$ & $-0.306(0.003)$ & $0.144(0.148)$ & $0.274(0.006)$ & $0.085(0.374)$ & $0.107(0.264)$ & $-0.160(0.101)$ \\
\hline
\end{tabular}


Table 4. Correlation between DKK1, FRZB, GREM1 with pro-inflammatory factors and painfulness. Spearman coefficient $(\mathrm{R})$ and $\mathrm{P}$ values are indicated. Significant correlations are bolded. Borderline significant are bolded and italic. FRZB, frizzled-related protein; GREM1, Gremlin 1; DKK1, Dickkopf-related protein; SF, synovial fluid; Se, serum; IL, interleukin; TNF, tumor necrosis factor; PGE2: Prostaglandin-E2; WOMAC: Western Ontario and McMaster Universities Arthritis Index.

\section{Discussion}

Due to the poor self-healing ability of articular cartilage and lack of specific diagnostic biomarkers, identification of patients at risk for OA or disease progression remains a challenge. Accumulating evidence shows that the activation of WNT signal pathways might be a driving factor for initiation and progression of OA, which makes the proteins of WNT signaling pathways attractive targets for therapy and diagnosis. Although large research effort has been put to identify the best strategy in targeting WNT signaling, the challenge remains unsolved due to the complexity of OA disease. A more promising approach will be identifying the dysregulation of WNT proteins or their natural inhibitors that are involved in OA [38]. In vivo and in vitro studies have shown that the natural antagonists of WNT signaling, such as DKK1 and FRZB, play a crucial role in joint homeostasis, especially through their protective role by inhibiting WNT signaling in joints $[26,39,40]$. This make us to postulate that their expression might change during OA pathology and any treatment increasing their expression might help to slow OA process by inhibiting WNT signaling. In the present study, we investigated the concentration of DKK1, FRZB and GREM1 in synovial fluid and serum of OA patients, and correlated their expression with OA pathological features and joint inflammation.

The patients recruited to this study had severe OA and received in vivo pharmaceutical treatment shortly before joint replacement surgery. We found that celecoxib treatment has beneficial effects on FRZB expression. It has been reported that celecoxib, which is a selective cyclooxygenase (COX)-2 has positive effects on human OA cartilage [41, 42]. Ample evidence 
confirms that genetic variation in the FRZB gene is associated with either hip OA or OA at multiple joint sites $[21,22,43]$, indicating that FRZB is important for joint homeostasis. The induction effect of celecoxib on FRZB may suggest that the chondro-protective role of celecoxib is partially mediated by inducing anabolic factors like FRZB.

Synovial tissue, cartilage, synovial fluid and serum were obtained from OA patients at joint replacement surgery. The correlation between the expression level of three factors (DKK1, FRZB and GREM1) and actual cartilage damage and inflammation was analyzed. We found that DKK1 conc. in SF negatively correlated with the severity of OA, which is in accordance with the evidence that DKK1 is associated with OA development and that high levels of DKK1 may play a protective role against cartilage degeneration [44]. Similarly, FRZB in SF negatively correlated with OA severity scores as well. Moreover, FRZB in synovial fluid has the same expression trend as in serum. This is in line with previous studies showing that loss of FRZB contributes to cartilage damage by increasing MMP expression and enhancing their activity [40]. Additionally, FRZB has a protective role on cartilage degradation and MMP induction by attenuating deleterious effects of the activation of the canonical WNT/ $\beta$-catenin pathway [39]. Interestingly, we found the conc. of FRZB both in SF and serum significantly correlated with DKK1 in SF. This indicates that DKK1 and FRZB might work synergistically to block high activity of WNT signaling in OA.

Synovial inflammation or synovitis is a frequently observed phenomenon in end stage osteoarthritic joints and contributes to the pathogenesis of OA through formation of various catabolic and pro-inflammatory mediators such as IL1 $\beta$, TNF $\alpha$, NO and PGE2 [45]. These mediators are produced by the inflamed synovium and alter the balance of cartilage matrix degradation and repair, leading to excess production of the proteolytic enzymes responsible for cartilage breakdown [46]. It has been shown that DKK1 is anti-inflammatory by inhibiting multiple signaling pathways in pericytes and myofibroblasts [47] and expression of DKK1 and FRZB is downregulated by IL1 $\beta$ in human chondrocytes [27]. In our study, DKK1 in SF was negatively correlated with $\mathrm{NO} /$ cartilage $(=0.001)$ and IL1 $\beta$ /weight synovium $(\mathrm{P}=0.059)$; SF FRZB negatively correlated with PGE2/weight cartilage $(\mathrm{P}=0.041), \mathrm{IL} 1 \beta /$ weight synovium 
( $\mathrm{P}=0.096), \mathrm{PGE} 2 /$ weight synovium (0.064) and NO/weight synovium ( $\mathrm{P}=0.047)$ (Fig. 5). This suggests that the high level of inflammatory factors during OA might cause the loss of WNT antagonists DKK1 and FRZB, resulting in the activation of $\beta$-catenin in chondrocytes, which may consequently contribute to OA development.

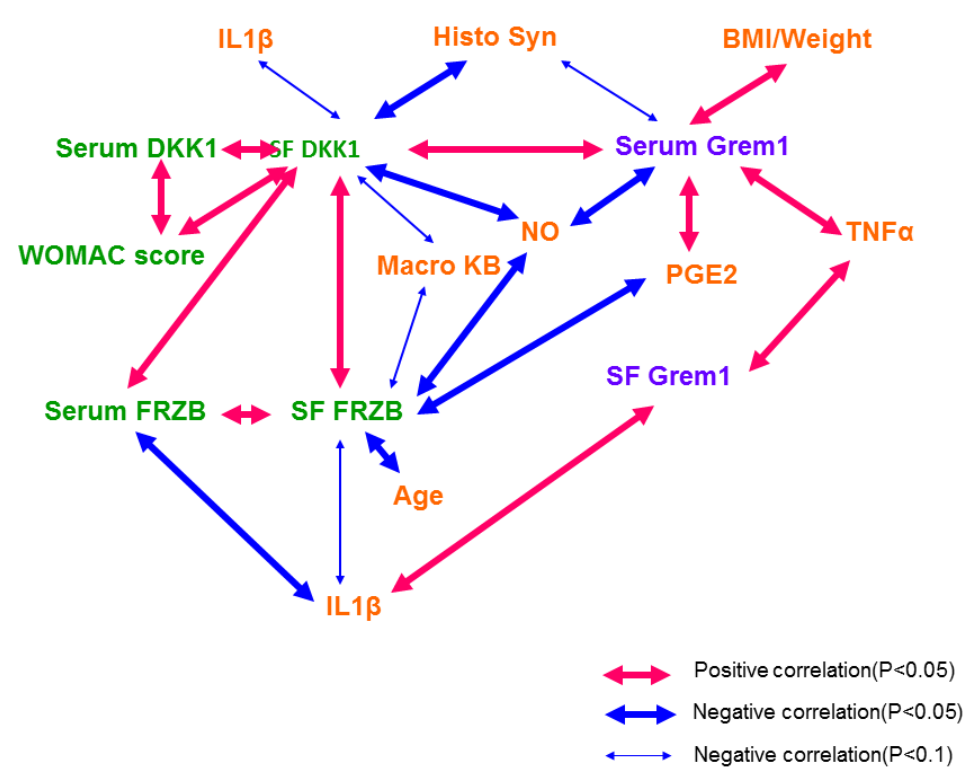

Fig. 5 Correlation between DKK1, FRZB, GREM1 and pro-inflammatory factors. Thick red arrows show positive correlation $(\mathrm{P}<0.05)$, thick blue arrows show negative correlation $(\mathrm{P}<0.05)$, light blue arrows show negative correlation $(\mathrm{P}<0.1)$. FRZB, frizzled-related protein; GREM1, Gremlin 1; DKK1, Dickkopf-related protein; IL, interleukin; TNF, tumor necrosis factor $\alpha$; PGE2: ProstaglandinE2; WOMAC: Western Ontario and McMaster Universities Arthritis Index; Macro KB; Histo Syn; BMI; Data from Table 4.

Pain and loss of function is a frequent symptom in OA patients. Studies have shown that the presence of local joint inflammation and the local release of pro-inflammatory mediators such as prostaglandins and cytokines accompanied by the destruction of tissue, may contribute to the pain [48]. WOMAC questionnaire was used to measure the clinical severity of the disease and scored on a 0-100 scale. Low score means more pain, stiffness and functional limitation. 
Interestingly, our data show that the expression of DKK1 in SF and serum positively correlated with WOMAC total score, suggesting that DKK1 somehow benefit to OA patient.

GREM1 as a BMP signal antagonist, is also involved in chondrocyte differentiation and cartilage homeostasis $[26,49]$. GREM1 has been shown to be highly expressed in fibroblastlike synoviocytes in rheumatoid arthritis (RA), contributes to synovial hyperplasia and its expression is induced by pro-inflammatory factors. In addition, GREM1 SF concentration correlates well with IL1 $\beta$, IL16 and TGF $\beta$ [50]. Synovial hyperplasia is also observed in OA [51] and microarray technology has shown that GREM1 is significantly up-regulated in OA chondrocytes [28]. In our study, we found that synovial fluid GREM1 positively correlated with IL1 $\beta /$ weight synovium, TNF $\alpha /$ weight synovium. Moreover, serum GREM1 positively correlated with PGE2/weight cartilage, TNF $\alpha$ /weight synovium, weight and BMI. These results indicate that the inflammatory environment in OA might contribute to high GREM1 expression, further lead to synovial hyperplasia.

Taking together, our data provide further evidence for the important involvement of DKK1, FRZB and GREM1 in OA and shows that their expression is associated with inflammation factors. This suggests that DKK1 and FRZB can be potential biomarkers for joint disease. To our knowledge, this is the first investigation of expression levels of DKK1, FRZB and GREM1 from synovial fluid and paired serum simultaneously together with an evaluation of their relationship with pro-inflammatory cytokines and actual cartilage damage degree as evaluated by macroscopy and histology. 


\section{References}

1. Buckland, J., Osteoarthritis: targeting cartilage erosion in OA. Nat Rev Rheumatol, 2010. 6(2): p. 64.

2. Ishiguro, N., T. Kojima, and A.R. Poole, Mechanism of cartilage destruction in osteoarthritis. Nagoya J Med Sci, 2002. 65(3-4): p. 73-84.

3. Kawaguchi, H., Endochondral ossification signals in cartilage degradation during osteoarthritis progression in experimental mouse models. Mol Cells, 2008. 25(1): p. 16.

4. Westacott, C., Interactions between subchondral bone and cartilage in OA. Cells from osteoarthritic bone can alter cartilage metabolism. J Musculoskelet Neuronal Interact, 2002. 2(6): p. 507-9.

5. Corr, M., Wnt-beta-catenin signaling in the pathogenesis of osteoarthritis. Nat Clin Pract Rheumatol, 2008. 4(10): p. 550-6.

6. Zhai, G., J. Dore, and P. Rahman, TGF-beta signal transduction pathways and osteoarthritis. Rheumatol Int, 2015. 35(8): p. 1283-92.

7. Rigoglou, S. and A.G. Papavassiliou, The NF-kappaB signalling pathway in osteoarthritis. Int J Biochem Cell Biol, 2013. 45(11): p. 2580-4.

8. Xue, F., et al., Analysis of critical molecules and signaling pathways in osteoarthritis and rheumatoid arthritis. Mol Med Rep, 2013. 7(2): p. 603-7.

9. Usami, Y., et al., Wnt signaling in cartilage development and diseases: lessons from animal studies. Lab Invest, 2016. 96(2): p. 186-96.

10. Salazar, V.S., L.W. Gamer, and V. Rosen, BMP signalling in skeletal development, disease and repair. Nat Rev Endocrinol, 2016. 12(4): p. 203-21.

11. Yasuhara, R., et al., Roles of beta-catenin signaling in phenotypic expression and proliferation of articular cartilage superficial zone cells. Lab Invest, 2011. 91(12): p. 1739-52. 
12. Nakamura, Y., M. Nawata, and S. Wakitani, Expression profiles and functional analyses of Wnt-related genes in human joint disorders. Am J Pathol, 2005. 167(1): p. 97-105.

13. Zhu, M., et al., Activation of beta-catenin signaling in articular chondrocytes leads to osteoarthritis-like phenotype in adult beta-catenin conditional activation mice. J Bone Miner Res, 2009. 24(1): p. 12-21.

14. Sen, M., et al., Expression and function of wingless and frizzled homologs in rheumatoid arthritis. Proc Natl Acad Sci U S A, 2000. 97(6): p. 2791-6.

15. van Beuningen, H.M., et al., Differential effects of local application of BMP-2 or TGFbeta 1 on both articular cartilage composition and osteophyte formation. Osteoarthritis Cartilage, 1998. 6(5): p. 306-17.

16. Papathanasiou, I., K.N. Malizos, and A. Tsezou, Bone morphogenetic protein-2induced Wnt/beta-catenin signaling pathway activation through enhanced low-densitylipoprotein receptor-related protein 5 catabolic activity contributes to hypertrophy in osteoarthritic chondrocytes. Arthritis Res Ther, 2012. 14(2): p. R82.

17. Nakase, T., et al., Localization of bone morphogenetic protein-2 in human osteoarthritic cartilage and osteophyte. Osteoarthritis Cartilage, 2003. 11(4): p. 27884.

18. Haaijman, A., et al., Inhibition of terminal chondrocyte differentiation by bone morphogenetic protein $7(O P-1)$ in vitro depends on the periarticular region but is independent of parathyroid hormone-related peptide. Bone, 1999. 25(4): p. 397-404.

19. Caron, M.M., et al., Hypertrophic differentiation during chondrogenic differentiation of progenitor cells is stimulated by BMP-2 but suppressed by BMP-7. Osteoarthritis Cartilage, 2013. 21(4): p. 604-13.

20. Loughlin, J., et al., Functional variants within the secreted frizzled-related protein 3 gene are associated with hip osteoarthritis in females. Proc Natl Acad Sci U S A, 2004. 101(26): p. 9757-62.

21. Lane, N.E., et al., Frizzled-related protein variants are risk factors for hip osteoarthritis. Arthritis Rheum, 2006. 54(4): p. 1246-54. 
22. Min, J.L., et al., Association of the Frizzled-related protein gene with symptomatic osteoarthritis at multiple sites. Arthritis Rheum, 2005. 52(4): p. 1077-80.

23. Valdes, A.M., et al., Sex and ethnic differences in the association of ASPN, CALM1, COL2A1, COMP, and FRZB with genetic susceptibility to osteoarthritis of the knee. Arthritis Rheum, 2007. 56(1): p. 137-46.

24. Lories, R.J., et al., Evidence for a differential association of the Arg200Trp singlenucleotide polymorphism in FRZB with hip osteoarthritis and osteoporosis. Rheumatology (Oxford), 2006. 45(1): p. 113-4.

25. Lane, N.E., et al., Wnt signaling antagonists are potential prognostic biomarkers for the progression of radiographic hip osteoarthritis in elderly Caucasian women. Arthritis Rheum, 2007. 56(10): p. 3319-25.

26. Leijten, J.C., et al., Gremlin 1, frizzled-related protein, and Dkk-1 are key regulators of human articular cartilage homeostasis. Arthritis Rheum, 2012. 64(10): p. 3302-12.

27. Leijten, J.C., et al., GREM1, FRZB and DKK1 mRNA levels correlate with osteoarthritis and are regulated by osteoarthritis-associated factors. Arthritis Res Ther, 2013. 15(5): p. R126.

28. Tardif, G., et al., Differential gene expression and regulation of the bone morphogenetic protein antagonists follistatin and gremlin in normal and osteoarthritic human chondrocytes and synovial fibroblasts. Arthritis Rheum, 2004. 50(8): p. 2521-30.

29. Tardif, G., et al., The BMP antagonists follistatin and gremlin in normal and early osteoarthritic cartilage: an immunohistochemical study. Osteoarthritis Cartilage, 2009. 17(2): p. 263-70.

30. Goldring, M.B. and M. Otero, Inflammation in osteoarthritis. Curr Opin Rheumatol, 2011. 23(5): p. 471-8.

31. Houard, X., M.B. Goldring, and F. Berenbaum, Homeostatic mechanisms in articular cartilage and role of inflammation in osteoarthritis. Curr Rheumatol Rep, 2013. 15(11): p. 375 .

32. Heinegard, D. and T. Saxne, The role of the cartilage matrix in osteoarthritis. Nat Rev Rheumatol, 2011. 7(1): p. 50-6. 
33. Sumer, E.U., et al., Application of biomarkers in the clinical development of new drugs for chondroprotection in destructive joint diseases: a review. Biomarkers, 2006. 11(6): p. $485-506$.

34. Sandell, L.J. and T. Aigner, Articular cartilage and changes in arthritis. An introduction: cell biology of osteoarthritis. Arthritis Res, 2001. 3(2): p. 107-13.

35. Poole, A.R., et al., Changes in cartilage metabolism in arthritis are reflected by altered serum and synovial fluid levels of the cartilage proteoglycan aggrecan. Implications for pathogenesis. J Clin Invest, 1994. 94(1): p. 25-33.

36. EM, V.H., et al., Lack of a Clear Disease Modifying Activity of Celecoxib Treatment of End-Stage Knee Osteoarthritis: A Randomized Observer Blinded Clinical Trial. JSM Bone and Joint Dis, 2017. 1(2): p. 1006.

37. Bellamy, N., et al., Validation study of WOMAC: a health status instrument for measuring clinically important patient relevant outcomes to antirheumatic drug therapy in patients with osteoarthritis of the hip or knee. J Rheumatol, 1988. 15(12): p. 1833-40.

38. Blom, A.B., et al., To seek shelter from the WNT in osteoarthritis? WNT-signaling as a target for osteoarthritis therapy. Curr Drug Targets, 2010. 11(5): p. 620-9.

39. Bougault, C., et al., Protective role of frizzled-related protein $B$ on matrix metalloproteinase induction in mouse chondrocytes. Arthritis Res Ther, 2014. 16(4): p. R137.

40. Lories, R.J., et al., Articular cartilage and biomechanical properties of the long bones in Frzb-knockout mice. Arthritis Rheum, 2007. 56(12): p. 4095-103.

41. El Hajjaji, H., et al., Celecoxib has a positive effect on the overall metabolism of hyaluronan and proteoglycans in human osteoarthritic cartilage. J Rheumatol, 2003. 30(11): p. 2444-51.

42. de Boer, T.N., et al., The chondroprotective effect of selective COX-2 inhibition in osteoarthritis: ex vivo evaluation of human cartilage tissue after in vivo treatment. Osteoarthritis Cartilage, 2009. 17(4): p. 482-8.

43. Rodriguez-Lopez, J., et al., Further evidence of the role of frizzled-related protein gene polymorphisms in osteoarthritis. Ann Rheum Dis, 2007. 66(8): p. 1052-5. 
44. Voorzanger-Rousselot, N., N.C. Ben-Tabassi, and P. Garnero, Opposite relationships between circulating Dkk-1 and cartilage breakdown in patients with rheumatoid arthritis and knee osteoarthritis. Ann Rheum Dis, 2009. 68(9): p. 1513-4.

45. Sutton, S., et al., The contribution of the synovium, synovial derived inflammatory cytokines and neuropeptides to the pathogenesis of osteoarthritis. Vet J, 2009. 179(1): p. $10-24$.

46. Sellam, J. and F. Berenbaum, The role of synovitis in pathophysiology and clinical symptoms of osteoarthritis. Nat Rev Rheumatol, 2010. 6(11): p. 625-35.

47. Ren, S., et al., LRP-6 is a coreceptor for multiple fibrogenic signaling pathways in pericytes and myofibroblasts that are inhibited by DKK-1. Proc Natl Acad Sci U S A, 2013. 110(4): p. 1440-5.

48. Sofat, N., V. Ejindu, and P. Kiely, What makes osteoarthritis painful? The evidence for local and central pain processing. Rheumatology (Oxford), 2011. 50(12): p. 2157-65.

49. Canalis, E., K. Parker, and S. Zanotti, Gremlin1 is required for skeletal development and postnatal skeletal homeostasis. J Cell Physiol, 2012. 227(1): p. 269-77.

50. Han, E.J., et al., GREM1 Is a Key Regulator of Synoviocyte Hyperplasia and Invasiveness. J Rheumatol, 2016. 43(3): p. 474-85.

51. Scanzello, C.R. and S.R. Goldring, The role of synovitis in osteoarthritis pathogenesis. Bone, 2012. 51(2): p. 249-57. 
Table S1. Technical performance of the DKK1, FRZB and GREM1 immunoassays using synovial fluid. To calculate dilution linearity randomly selected synovial fluid samples $(n=3)$ were prepared in three different dilutions and analyzed. Results are expressed as \% recovery: $100 \times$ [(concentration at a specific dilution) divided by (first diluted concentration divided by the dilution times)]. Spiking recovery was calculated from randomly selected synovial fluid samples $(n=3)$ which were spiked using high, middle or low concentrations of standards. Spiking recovery is expressed in $\%$ as recovery: 100x [(concentration of a sample spiked with a specific amount of standard or another sample) divided by (the individual concentration of sample plus the individual concentration from standard or another sample)]. To analyze freeze-thawing effects on FRZB and GREM1 concentrations, randomly selected synovial fluid samples $(n=3)$ were freeze/thawed 3 times. Recovery after freeze-thaw cycles is expressed in relation to the amount obtained the first time the sample was thawed. Intra and inter DKK1. FRZB and GREM1 assay coefficient of variations (CV) was calculated using randomly selected synovial fluid samples $(n=3)$ loading 5 repeats (intra) on a single plate or loading duplicates on 4 plates (inter). Lower limit of detection (LLOD) and upper limit of detection (ULOD) for the DKK1 FRZB and GREM1 assays were estimated. $\mathrm{Na}=$ not applicable due to either to low or to high dilution ratio. 


\begin{tabular}{|c|c|c|c|c|c|}
\hline \multicolumn{2}{|l|}{ Analyte } & & DKK1 & FRZB & GREM1 \\
\hline \multirow{2}{*}{\multicolumn{2}{|c|}{$(\mathrm{pg} / \mathrm{ml})$}} & LLOD & 7.77 & 7.77 & 57 \\
\hline & & ULOD & 4000 & 8000 & 8000 \\
\hline \multicolumn{6}{|c|}{ Recovery. mean (range) \% } \\
\hline & Dilution & \multicolumn{4}{|l|}{ Ratio } \\
\hline & & $1: 2$ & $97(77-115)$ & $87(78-96)$ & $\mathrm{Na}$ \\
\hline & & $1: 4$ & $93(85-112)$ & $93(72-120)$ & $\mathrm{Na}$ \\
\hline & & $1: 8$ & 87 (76-106) & $91(71-113)$ & $\mathrm{Na}$ \\
\hline & & $1: 24$ & $\mathrm{Na}$ & $\mathrm{Na}$ & $114(97-131)$ \\
\hline & & $1: 48$ & $\mathrm{Na}$ & $\mathrm{Na}$ & $83(68-97)$ \\
\hline & & $1: 96$ & $\mathrm{Na}$ & $\mathrm{Na}$ & $98(82-119)$ \\
\hline & Spiking & \multicolumn{4}{|l|}{$n$} \\
\hline & High conc. & 3 or 4 & $90(87-94)$ & $92(83-110)$ & $102(81-124)$ \\
\hline & Middle conc. & 3 or 4 & $85(80-92)$ & $90(84-102)$ & $107(94-121)$ \\
\hline & Low conc. & 3 or 4 & $84(80-90)$ & $104(86-122)$ & 97 (84-115) \\
\hline & Freeze-thaw & \multicolumn{4}{|l|}{ cycle } \\
\hline & & $1^{\text {st }}$ & $106(98-115)$ & $93(91-103)$ & $98(89-106)$ \\
\hline & & $2^{\text {nd }}$ & $95(82-103)$ & $98(90-110)$ & 97 (86-116) \\
\hline & & $3^{\text {nd }}$ & $118(114-122)$ & $101(88-120)$ & $111(89-133)$ \\
\hline \multirow[t]{3}{*}{ CV \% } & & \multicolumn{4}{|l|}{ Assay } \\
\hline & & Intra & 6.6 & 5.2 & 8.3 \\
\hline & & Inter & 7.6 & 6.2 & 9.2 \\
\hline
\end{tabular}


Table S2. Technical performance of the DKK1, FRZB and GREM1 immunoassays using serum. To calculate dilution linearity randomly selected serum samples $(n=3)$ were prepared in three different dilutions and analyzed. Results are expressed as \% recovery: 100 x [(concentration at a specific dilution) divided by (first diluted concentration divided by the dilution times)]. Spiking recovery was calculated from randomly selected serum samples $(n=3)$ which were spiked using high middle or low concentrations of standards. Spiking recovery is expressed in \% as recovery: 100x [(concentration of a sample spiked with a specific amount of standard or another sample) divided by (the individual concentration of sample plus the individual concentration from standard or another sample)]. To analyze freeze-thawing effects on DKK1, FRZB and GREM1 concentrations, randomly selected serum samples $(n=3)$ were freeze/thawed 3 times. Recovery after freeze-thaw cycles is expressed in relation to the amount obtained the first time the sample was thawed. Intra and inter DKK1. FRZB and GREM1 assay coefficient of variations $(\mathrm{CV})$ was calculated using randomly selected serum samples $(n=3)$ loading 5 repeats (intra) on a single plate or loading duplicates on 4 plates (inter). Lower limit of detection (LLOD) and upper limit of detection (ULOD) for the DKK1 FRZB and GREM1 assays were estimated. $\mathrm{Na}=$ not applicable due to either to low or to high dilution ratio. 


\begin{tabular}{|c|c|c|c|c|c|}
\hline \multicolumn{3}{|l|}{ Analyte } & \multirow{2}{*}{$\begin{array}{l}\text { DKK1 } \\
7.77\end{array}$} & \multirow{2}{*}{$\begin{array}{l}\text { FRZB } \\
7.77\end{array}$} & \multirow{2}{*}{$\begin{array}{l}\text { GREM1 } \\
57\end{array}$} \\
\hline$(\mathrm{pg} / \mathrm{ml})$ & & LLOD & & & \\
\hline & & ULOD & 4000 & 8000 & 8000 \\
\hline \multicolumn{6}{|c|}{ Recovery. mean (range) \% } \\
\hline & Dilution & Ratio & & & \\
\hline & & $1: 2$ & $115.9(114-119.3)$ & $108.8(96.7-120.9)$ & $\mathrm{Na}$ \\
\hline & & $1: 4$ & $107.6(92.1-117.1)$ & $79(71-87)$ & $\mathrm{Na}$ \\
\hline & & $1: 8$ & $79.7(76.8-83.4)$ & $91.7(72.7-115)$ & $\mathrm{Na}$ \\
\hline & & $1: 24$ & $\mathrm{Na}$ & $\mathrm{Na}$ & $91.3(82.1-109.1)$ \\
\hline & & $1: 48$ & $\mathrm{Na}$ & $\mathrm{Na}$ & $94(77.6-113)$ \\
\hline & & $1: 96$ & $\mathrm{Na}$ & $\mathrm{Na}$ & $113(97.7-121.6)$ \\
\hline & Spiking & $n$ & & & \\
\hline & High conc. & 3 & $89.3(84-97)$ & $91.5(76.2-101.3)$ & $103.2(75.2-117.1)$ \\
\hline & Middle conc. & 3 & $74.1(70.5-82.3)$ & $75(69.2-84.5)$ & $86.1(73.7-104.1)$ \\
\hline & Low conc. & 3 & $82.7(78.8-87.8)$ & $80.9(79.8-82.3)$ & $108.5(101.3-115)$ \\
\hline & Freeze-thaw & cycle & & & \\
\hline & & $1^{\mathrm{st}}$ & $107.3(98.5-112)$ & $96.3(74.3-125.8)$ & $113.1(104.6-124)$ \\
\hline & & $2^{\text {nd }}$ & $100.3(92-111.8)$ & $102.1(91.7-114.3)$ & $99.3(84.7-124.2)$ \\
\hline & & $3^{\text {nd }}$ & $96.4(82.5-113.5)$ & $110.7(79.8-128.9)$ & $107.4(84.4-120.3)$ \\
\hline \multirow[t]{3}{*}{ CV \% } & & Assay & & & \\
\hline & & Intra & 8.6 & 7.4 & 8.5 \\
\hline & & Inter & 8.9 & 9.4 & 10 \\
\hline
\end{tabular}




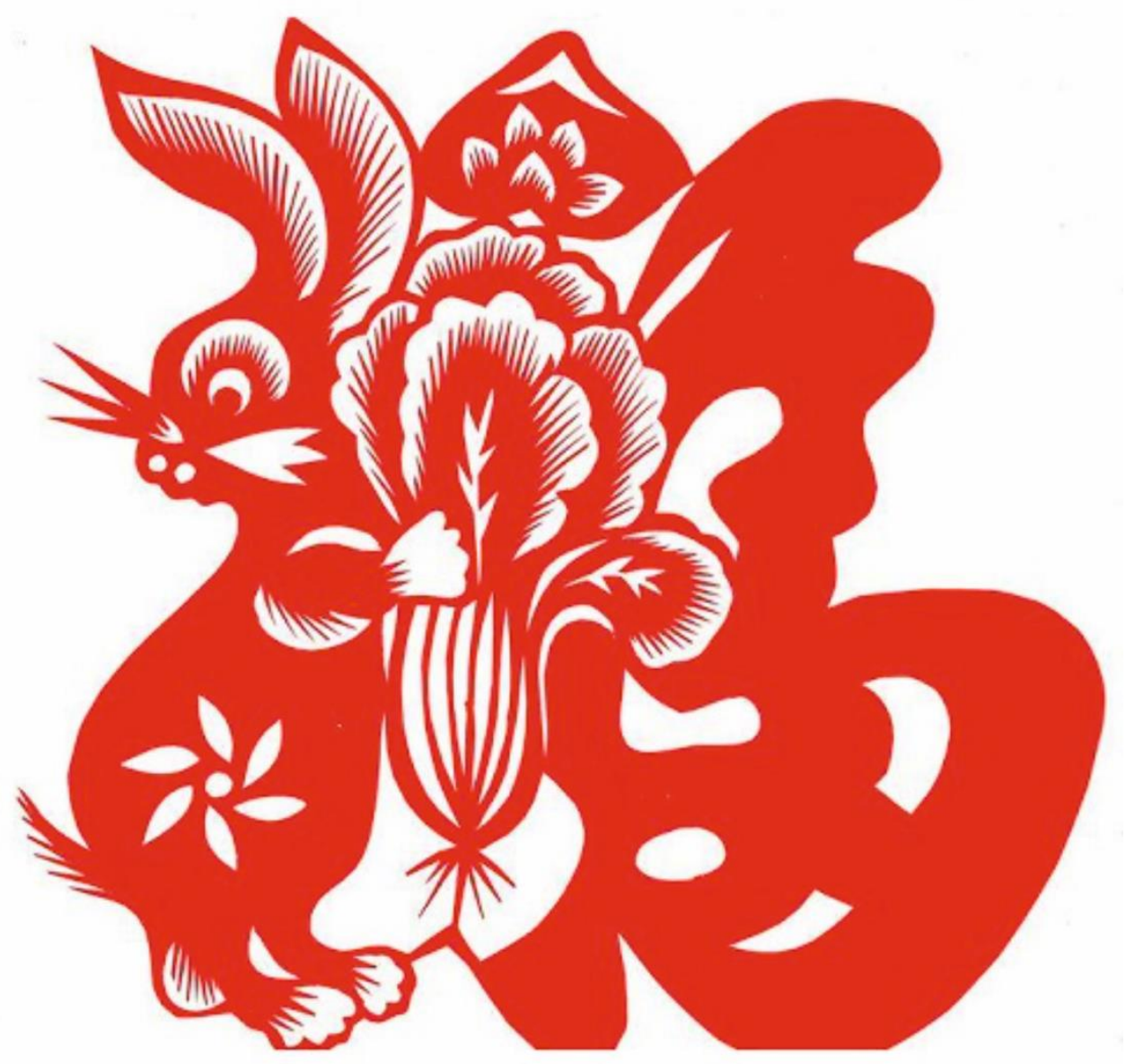


Chapter 4

The effects of the WNT-signaling modulators BIO and PKF118310 on the chondrogenic differentiation of human mesenchymal stem cells

Xiaobin Huang, Leilei Zhong, Jan Hendriks, Janine N. Post and Marcel Karperien

Developmental BioEngineering, MIRA Institute for Biomedical Technology and Technical Medicine, University of Twente, Enschede 7500 AE, The Netherlands

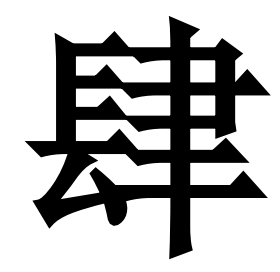

天长地久, 天地所以能长且久者, 以其不自生, 故能长生。

Heaven is eternal, the Earth Everlasting. How come they to be so?

It is because they do not foster their own lives. That is why they live so long. 


\section{Abstract}

Objective: Mesenchymal stem cells (MSCs) are multipotent cells, mainly from bone marrow, and an ideal source of cells in bone and cartilage tissue engineering. A study of the chondrogenic differentiation of MSCs is of particular interest for MSCs-based cartilage regeneration. In this study, we aimed to optimize the conditions for the chrondogenic differentiation of MSCs by regulating WNT signaling using the small molecule WNT inhibitor PKF118-310 and activator BIO.

Methods: Human mesenchymal stem cells (hMSCs) were isolated from bone marrow aspirates and cultured in hMSCs proliferation medium. Pellet culture was subsequently established for 3-D chondrogenic differentiation of 5 weeks. WNT signaling was increased by the small molecule glycogen synthase kinase-3 inhibitor 6-bromoindirubin-3-oxim (BIO) and decreased by WNT inhibitor PKF118-310 (PKF). The effects of BIO and PKF on the chondrogenesis of hMSCs was examined by real-time PCR, histological methods and ELISA.

Results: Activation of canonical WNT-signaling by BIO significantly downregulated the expression of cartilage-specific genes SOX9, COL2AI and ACAN, and matrix metalloproteinase genes MMP1/3/9/13, but increased TIMP3 and ADAMTS 4/5. Inhibition of WNT signaling by PKF increased the expression of SOX9, COL2A1, ACAN, and MMP9, but decreased MMP13 and ADAMTS4/5. In addition, a high level of WNT signaling induced the expression of hypertrophic markers COL10A1, ALPL, RUNX2 and dedifferentiation marker COL1A1, as well as glycolysis genes GULT1 and PGK1. Deposition of glycosaminoglycan (GAG) and collagen type II in the pellet matrix was significantly lost in the BIO treated group and increased in the PKF treated group. The protein level of COL10A1 was also highly induced in the BIO group. Interestingly, BIO decreased the number of apoptotic cells while PKF significantly induced apoptosis during chondrogenesis. The natural WNT antagonist DKK1 and the protein level of MMP1 in the pellet culture medium were decreased after PKF treatment. All these chondrogenic effects appeared to be mediated through the canonical WNT signaling pathway since the target gene Axin2, and other WNT members such as TCF4 and $\beta$-catenin, were up-regulated by $\mathrm{BIO}$ and down-regulated by PKF respectively, and BIO induced nuclear translocation of $\beta$-catenin while PKF inhibited $\beta$-catenin translocation into nucleus. 
Conclusion: Addition of BIO to a chondrogenic medium of hMSCs resulted in loss of cartilage formation, while PKF induced chondrogenic differentiation, cartilage matrix deposition and inhibited hypertrophic differentiation. However, BIO promoted cell survival by inhibiting apoptosis while PKF induced cell apoptosis. This result indicates that either over-expression or over-inhibition of WNT signaling to some extent causes harmful effects on chondrogenic differentiation. Cartilage tissue engineering could benefit from the adjustment of the critical level of WNT signaling during chondrogenesis of hMSC. 


\section{Introduction}

The treatment of injuries to hyaline cartilage is considered a challenge, due to the limited ability of self-repair of articular cartilage. Although much research effort has been made to improve the regeneration of articular cartilage, there is no curative treatment available yet for rebuilding native articular cartilage. Human mesenchymal stem cells (hMSCs) are multipotent cells mainly from the bone marrow. They can be expanded in vitro and be differentiated into the osteogenic, chondrogenic, and adipogenic lineages [1], and are considered suitable candidates for the promotion of tissue regeneration [2, 3]. A study of cartilage differentiation of MSCs would be of particular interest for the cell-based treatment of cartilage defects, and optimization of MSCs chondrogenesis is of particular importance. Since WNT signaling is indicated to be involved in the chondrogenic differentiation of hMSCs, the chondrogenic process can be optimized by regulating WNT signaling.

The WNT signaling pathway is composed of several conserved components and plays a fundamental role in controlling cell proliferation, cell fate determination, and cell differentiation during embryonic development and in adult cartilage $[4,5]$. In an inactive state, due to the absence of WNT ligand, $\beta$-catenin is phosphorylated at the $\mathrm{NH}_{2}$ terminus by glycogen synthase kinase (GSK) $3 \beta$ and casein kinase I (CKI) into a so-called destruction complex. This destruction complex is brought together by two scaffolding proteins, AXIN and adenomatous polyposis coli (APC). The phosphorylated $\beta$-catenin undergoes subsequent ubiquinitylation and proteasomal degradation. Conversely, in an active state, WNT ligands bind to frizzled receptors and its co-receptor of LDL related proteins (LRP) 5 or 6. This activates disheveled (DSH) subsequently inhibits GSK3 $\beta$ activity. As a result, $\beta$-catenin will not undergo degradation, and will accumulate followed by translocation into the nucleus. In the nucleus, it interacts with transcription factors of the $\mathrm{T}$ cell specific transcription factor/lymphoid enhancer-binding factor (TCF/LEF) family to initiate the transcription of target genes $[6,7]$. GSK3 $\beta$ is a key molecular regulator of the WNT pathway, since its inhibition initiates the signaling pathway [8-10].

Several small molecules that regulate WNT signaling have been reported [11]. In our study, we focus on two important regulators: BIO and PKF118-310 (PKF). BIO activates canonical WNT signaling in human chondrocytes by blocking GSK-3 $\beta$ [12]. The small molecule PKF is an inhibitor of WNT signaling by interfering with the binding of $\beta$-catenin to the transcription factor TCF4 [13-15]. 
The potential effects of small molecules that activate/inhibit WNT/ $\beta$-catenin signaling on cell culture has been demonstrated. Some studies reported that the presence of BIO in the culture medium enhances the growth capacity of the cultured cells [16-19]. BIO supplementation leads to the maintenance of pluripotency in embryonic stem cell culture $[19,20]$. A recent study has shown that the addition of $0.01 \mu \mathrm{M}$ BIO could accelerate and enhance in vitro chondrogenesis of mouse bone marrow-derived MSCs [21]. A previous study by our group has suggested that the inhibition of WNT/ $\beta$-catenin signaling by small molecules can effectively prevent IL1 $\beta$ and $\mathrm{TNF} \alpha$-induced cartilage degradation by blocking MMP expression and activity [22]. PKF, an inhibitor of WNT/ $\beta$-catenin signaling, inhibits the growth of HCC cells as well as targets breast tumor-initiating cells in a mouse model of breast cancer in vitro and in vivo [14, 23, 24].

Even though many studies focus on small inhibitors or activators of WNT/ $\beta$-catenin signaling in cell research, there is no report about the effect of inhibitor/activator on hMSCs chondrogenesis in vitro. The aim of this study was to assess the potential effects of small molecules on the chondrogenic differentiating hMSCs with continuous inhibition/activation of canonical WNT signaling, using a 3-D pellet culture system.

\section{Materials and methods}

\section{Luciferase assay}

HEK293t cells were infected with Cignal $^{\mathrm{TM}}$ lentiviruses containing a TCF/LEF luciferase reporter construct (SA Biosciences) together with lentiviruses constitutively expressing Renilla luciferase (SA Biosciences). HEK293t cells were seeded at 8000 cells $/ \mathrm{cm}^{2}$ in 96 -wells plates (Nunc international), and cultured for 24 hours in basic medium (DMEM supplemented with $10 \% \mathrm{FBS}$ and 100u/ml penicillin and streptomycin). The cells were treated with BIO (Sigma Aldrich) or PKF118-310 (PKF) (Sigma Aldrich) for 24 hours. After 24 hours, the cells were lysed in Glo lysis buffer and luciferase activity was measured using a Dual-Glo luciferase assay kit (Promega).

\section{Cell culture and expansion}

The use of human bone marrow aspirates was approved by a local medical ethics committee and written informed consent by the donors was obtained. The aspirates were resuspended 
using a 20G needle, and plated at a density of 0.5 million mononucleated cells $/ \mathrm{cm}^{2}$. MSCs were selected by adherence in a proliferation medium ( $\alpha$-MEM supplemented with $10 \%$ fetal bovine serum, $1 \%$ L-glutamin, $0.2 \mathrm{mM}$ ascorbic acid, $100 \mathrm{U} / \mathrm{ml}$ penicillin, $10 \mathrm{mg} / \mathrm{ml}$ streptomycin and $1 \mathrm{ng} / \mathrm{ml} \mathrm{bFGF})$.

\section{Pellet culture and chondrogenic differentiation of hMSCs}

To obtain pellet-cultures, 250,000 MSCs at passage three were seeded in $10 \mathrm{ml}$ round bottom tubes in chondrogenic differentiation medium (DMEM supplemented with $40 \mu \mathrm{g} / \mathrm{mL}$ proline,

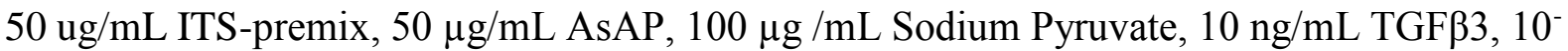

${ }^{7} \mathrm{M}$ dexamethasone, $100 \mathrm{U}$ penicillin $/ \mathrm{ml}$ and $100 \mu \mathrm{g} / \mathrm{ml}$ streptomycin) and centrifuged for $5 \mathrm{~min}$ at $500 \times \mathrm{g}$. Three groups were included in this experiment: hMSC pellets in chondrogenic differentiating medium as the control group, hMSC pellets with BIO treatment and hMSC pellets with PKF treatment. BIO and PKF were used at a concentration of $2 \mu \mathrm{M}$ and $3 \mu \mathrm{M}$ respectively. Three donors were used for each condition as biological triplicates. Cell pellets were grown at $37^{\circ}$ in a humid atmosphere with $5 \% \mathrm{CO} 2$. The medium was refreshed twice a week. The cell pellets were cultured for 5 weeks before analysis.

\section{RNA isolation and quantitative PCR}

RNA samples of cell pellets were isolated using TRIzol (Thermo Fisher Scientific) according to the manufacturer's protocol. The concentration and purity of the RNA samples were measured with the Nanodrop2000. The total RNA was reverse-transcribed into cDNA using the iScript cDNA Synthesis kit (Bio-Rad, Hercules, CA). Quantitative PCR (qPCR) was performed by using the SYBR Green sensimix (Bioline). PCR Reactions were carried out on a Bio-Rad CFX96 under the following conditions: the cDNA was denatured for 5 min at $95{ }^{\circ} \mathrm{C}$, followed by 45 cycles, consisting of $15 \mathrm{~s}$ at $95^{\circ} \mathrm{C}, 15 \mathrm{~s}$ at $60^{\circ} \mathrm{C}$ and $30 \mathrm{~s}$ at $72^{\circ} \mathrm{C}$. A melting curve was generated for each reaction to test primer dimer formation and non-specific priming. The gene expression was normalized using RPL13A and expressed as fold induction compared to controls.

\section{Alcian blue/Safranin $O$ and Alizarin red staining}

Chondrogenically differentiated hMSC pellets were fixed with $10 \%$ buffered formalin for 15 minutes and embedded in paraffin using routine procedures. Sections of $5 \mu \mathrm{m}$ were cut using a microtome (Shandon). Before staining, the slides were de-paraffinized in xylene and rehydrated with graded ethanols. Sulfated glycosaminoglycans (GAG) were stained with a $0.5 \%$ 
w/v solution of Alcian blue $(\mathrm{pH}=1$, adjusted by $\mathrm{HCl}$ ) or a $0.1 \%$ solution of Safranin O (Sigma Aldrich) for $30 \mathrm{~min}$ and $5 \mathrm{~min}$, respectively. The samples were then counterstained with $0.1 \%$ $\mathrm{w} / \mathrm{v}$ of nuclear fast red (dissolved in 5\% aluminum sulfate) or haematoxylin (dissolved in water) for $5 \mathrm{~min}$ and $1 \mathrm{~min}$, respectively, to visualize nuclei. The sections were also stained with Alizarin red staining for mineralization.

\section{Immunofluorescent (IF) staining for collagen type II}

For the immunofluorescent staining of collagen type II, $5 \mu \mathrm{M}$ sections were de-paraffinized in xylene and rehydrated with graded ethanols. The samples were pre-incubated with $5 \mu \mathrm{g} / \mathrm{mL}$ proteinase K (Sigma Aldrich) for $10 \mathrm{~min}$ at RT, followed by $1 \mathrm{mg} / \mathrm{mL}$ hyaluronidase (Sigma Aldrich) for $40 \mathrm{~min}$ at $37^{\circ} \mathrm{C}$. They were blocked in 5\% BSA in PBS for 1 hour, after which they were incubated overnight at $4^{\circ}$ with rabbit anti-human collagen II antibody (Abcam), which was diluted 1:100 in 5\% BSA in PBS. The cells were rinsed 3 times with PBST, 5 min/time. Then Alexa®Fluor 546-labelled goat anti-rabbit antibody in 5\% BSA in PBS was added and incubated for 2 hours at RT. The samples were rinsed with PBS, and mounting medium with DAPI was added. The slides were viewed by BD pathway ${ }^{\mathrm{TM}}$ confocal microscopy (BD BioSciences).

\section{Immunohistochemistry (IHC) of $\beta$-catenin and Collagen type X}

Immunohistochemistry staining of $\beta$-catenin and Collagen type $X$ were performed using $5 \mu \mathrm{m}$ sections. The sections were pre-incubated with $5 \mu \mathrm{g} / \mathrm{mL}$ proteinase K (Sigma Aldrich) for 10 min, followed by $1 \mathrm{mg} / \mathrm{mL}$ hyaluronidase (Sigma Aldrich) for $40 \mathrm{~min}$ at $37{ }^{\circ} \mathrm{C}$. Rabbit antihuman $\beta$-catenin antibody (Life span Biosciences) or mouse anti-human Collagen type $\mathrm{X}$ antibody (BIOCYC GmbH \& Co. KG, Cat. No. 2031501005 ) was diluted 1:100 in PBS and incubated overnight at $4{ }^{\circ} \mathrm{C}$. Non-immune controls underwent the same procedure without primary antibody incubation. The biotinylated secondary antibody was diluted 1:500 in 5\% BSA in PBS and incubated for 30 minutes at RT. HRP-Streptavidin was added and incubated for 30 minutes at RT. A DAB substrate kit (ab64238, Abcam) was used for visualization. The cell nucleus was counterstained with hematoxylin for Collage type X detection. Images were taken by using a Nanozoomer (Iwata City, Japan). 


\section{TUNEL assay}

Apoptosis of chondrocytes was detected using The DeadEnd ${ }^{\mathrm{TM}}$ fluorometric TUNEL System (Promega). Nuclei were counterstained with Hoechst 33342. Images were viewed by means of BD pathway confocal microscopy.

\section{GAG and DNA assay}

After 5 weeks of chondrogenic differentiation, the pellets were predigested in $250 \mu 1$ of Tris$\mathrm{HCl}$ buffer (0.05 M Tris, $1 \mathrm{mM} \mathrm{CaCl} 2, \mathrm{pH}$ 8.0) with $1 \mathrm{mg} / \mathrm{ml}$ proteinase $\mathrm{K}$ (Roche) for $16 \mathrm{hrs}$ at $56^{\circ} \mathrm{C}$. Diluted samples $(25 \mu \mathrm{l})$ were mixed with $150 \mu 1$ 1,9-dimethylmethylene blue (DMMB) dye solution (3.04 g/l glycine, $2.38 \mathrm{~g} / \mathrm{l} \mathrm{NaCl}$ and $20 \mathrm{mg} / \mathrm{l} \mathrm{DMMB}$ in distilled water) and absorbance was measured at $525 \mathrm{~nm}$. The relative cell number was determined by quantification of the total DNA using a QuantiFluor ${ }^{\circledR}$ dsDNA System kit (Promega), according to the manufacturer's instructions.

\section{Enzyme linked immunosorbent assay (ELISA)}

The culture medium for pellet samples was collected weekly. The protein concentration of DKK1 was measured using ELISA according to the manufacturer's guidelines (DKK1, R\&D system). Secreted MMP1 in medium was also measured by ELISA, using mouse anti-human MMP1 antibody (MAB901-SP, R\&D systems), which was followed by incubation with a rabbit anti-mouse antibody coupled to a peroxidase. The amount of HRP was developed by the addition of Tetramethylbenzidine (TMB, 1-Step Ultra TMB-ELISA, Thermo Scientific). The reactions were stopped by the addition of $\mathrm{H}_{2} \mathrm{SO}_{4}$ and measured at $450 \mathrm{~nm}$ of microplate reader.

\section{Statistical analysis}

For the experiments using human bone marrow, samples were obtained from three donors. Each experiment was performed in triplicate. Data is expressed as the mean \pm SD and analyzed by two-tailed student's t-tests or one-way ANOVA. P $<0.05$ was considered statistically significant.

\section{Results}

\section{The efficacy and specificity of BIO and PKF on the TCF/LEF reporter}

In order to test the efficacy and specificity of BIO and PKF in terms of activating/blocking WNT/ $\beta$-catenin signaling, dose-response experiments were performed in HEK293t cells 
transfected with the TCF/LEF reporter. The cells were cultured in the medium with or without $\mathrm{BIO} / \mathrm{PKF}$. We found a dose-dependent increase in reporter activity when the cells were treated with BIO. Maximal activation was found at $2 \mu \mathrm{M}$. PKF treatment resulted in a ten-fold decrease in reporter activity at a concentration of $3 \mu \mathrm{M}$ (Figure 1A). On the basis of this result, we adopted the optimal concentration of $\mathrm{BIO}$ and $\mathrm{PKF}$ of $2 \mu \mathrm{M}$ and $3 \mu \mathrm{M}$ respectively in all subsequent experiments.

A

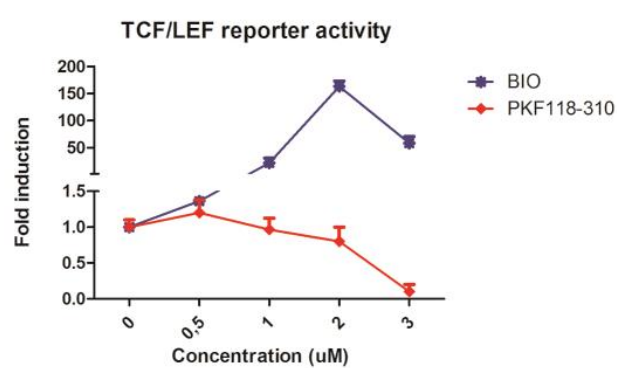

C

B
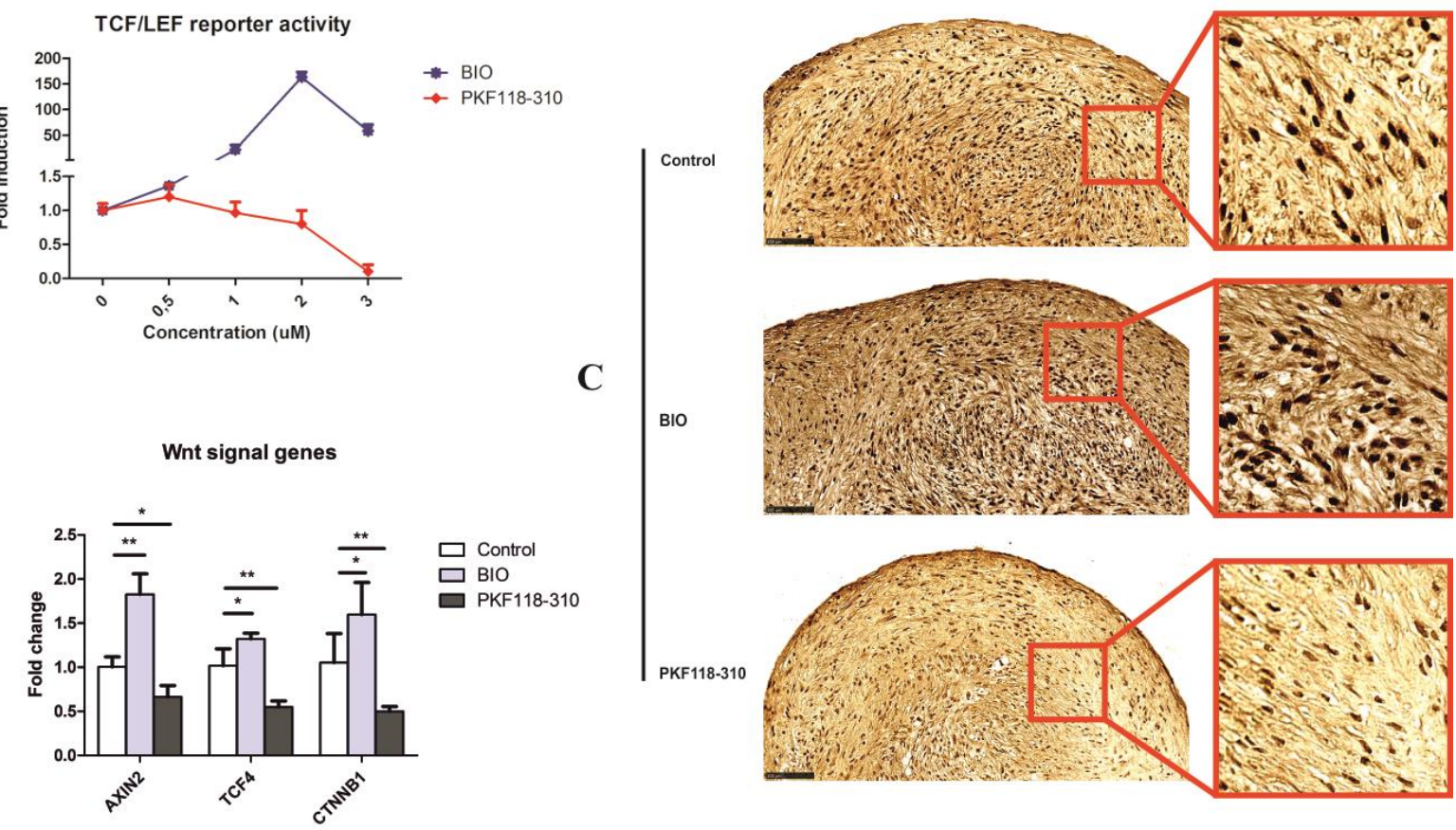

Figure 1 The efficacy and specificity of BIO and PKF on TCF/LEF reporter and hMSC. A. TCF/LEF reporter assay. To measure TCF/LEF reporter activity, HEK293T cells were infected with a lentiviral TCF/LEF reporter construct and stimulated with different concentrations of BIO or PKF for 24 hours, and luciferase activity was measured using the Dual-Glo luciferase assay kit. Data was expressed as fold change compared to control. B. The expression of WNT signaling responsive genes in hMSCs. $* \quad \mathrm{P}<0.05$. ** $\mathrm{P}<0.01$. Error bar represents Standard Deviation (SD). C. Immunohistochemistry of $\beta$-catenin in hMSC pellets. The expression of $\beta$-catenin was detected by rabbit anti-human $\beta$-catenin antibody, followed by incubation with the biotinylated secondary antirabbit antibody and HRP-Streptavidin, DAB substrate kit. Images were taken by using a Nanozoomer. Scale bar $=100 \mu \mathrm{m}$.

To verify whether the WNT signaling pathway is activated or inhibited by small molecules in hMSCs pellets, the effects of BIO and PKF118-130 on the expression of the WNT target gene AXIN2, and the WNT related genes TCF4 and CTNNB1 (gene encoding $\beta$-catenin protein), 
were measured by qPCR. The expression and nuclear translocation of $\beta$-catenin was detected by IHC. We observed that BIO significantly induced mRNA expression of the canonical WNT target gene AXIN2 and TCF4 and CTNNB1, while PKF effectively inhibited canonical WNT signaling (Figure 1B). IHC results showed that the cells had a basic $\beta$-catenin expression level. It was observed that BIO strongly enhanced the $\beta$-catenin expression and promoted its nucleus translocation, while PKF decreased the expression and inhibited nuclear translocation (Figure 1C). All these results suggested that canonical WNT signaling was efficiently activated by BIO and inhibited by PKF.

A

Chondrocyte markers mRNA

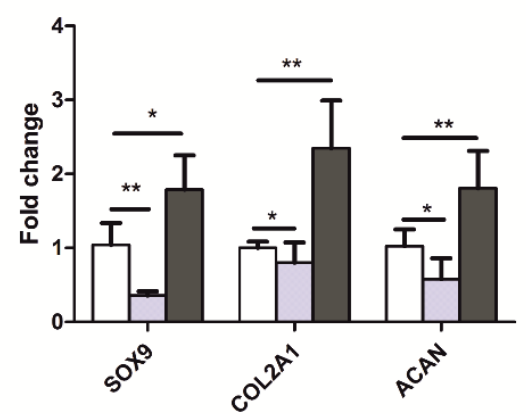

C

Catabolic genes mRNA

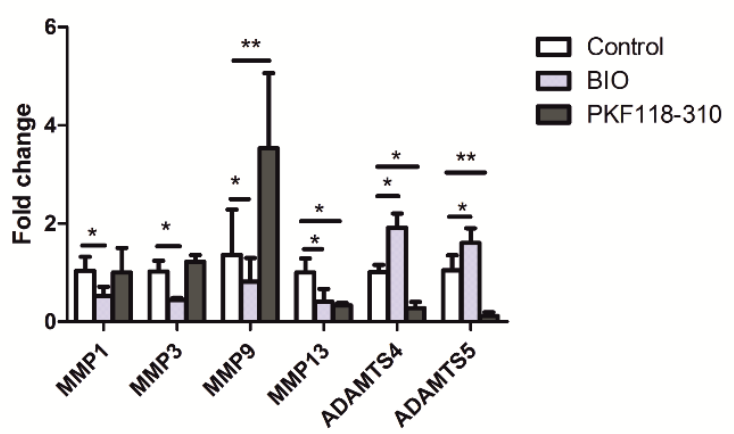

B Hypotrophic markers mRNA

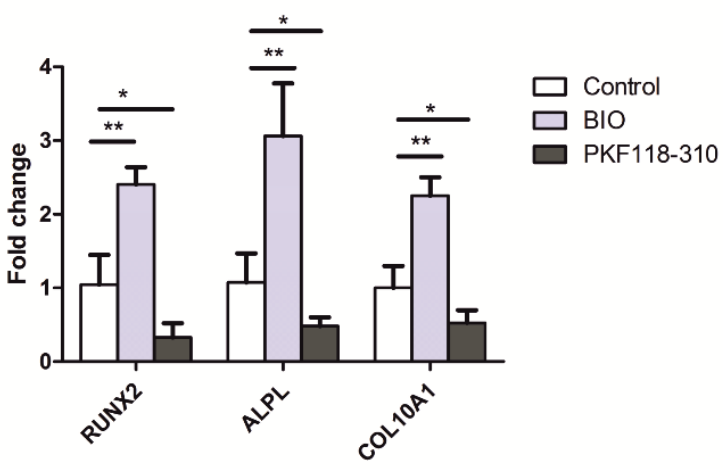

D

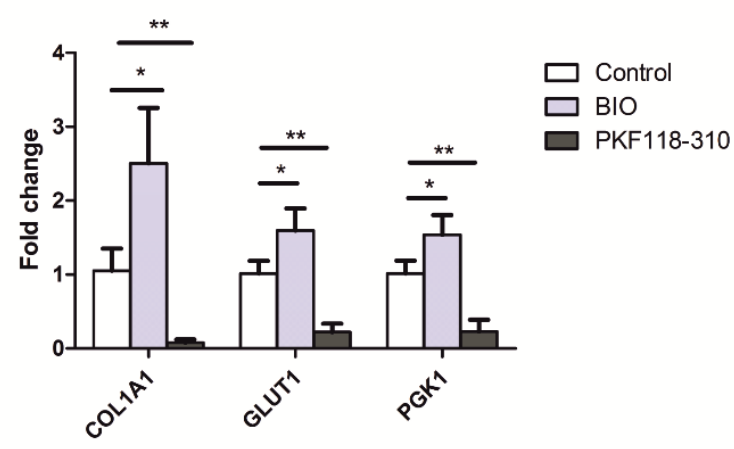

Figure 2 Gene expression in hMSCs pellets after activating/inhibiting canonical WNT signaling.

The expression of A. chondrocyte markers B. hypertrophic markers C. TIMP3 and catabolic genes D. COL1A1 and glycolysis genes. hMSCs pellets were cultured in chondrogenic differentiation medium with supplementation of $2 \mu \mathrm{M}$ BIO or $3 \mu \mathrm{M}$ PKF for 5 weeks. After 5 weeks, RNA samples were extracted from pellets and the expression of specific gene were analyzed by qPCR. Relative expression 
levels of different genes were normalized using RPL13A. Data are presented as an average of three hMSCs donors \pm SD. $* \mathrm{P}<0.05, * * \mathrm{P}<0.01$. Error bar represents Standard Deviation (SD).

\section{The effects of activation and inhibition of canonical WNT signaling on gene expression during hMSCs chondrogenesis}

To investigate the effects of canonical WNT signaling on chondrogenesis of hMSCs, the gene expression of chondrocyte markers was measured by qPCR after 5 weeks of pellet culture in the absence of presence of BIO or PKF. Expression of typical cartilage markers such as SOX9, COL2A1 and ACAN was significantly downregulated by BIO. However, Inhibition of WNT signaling by PKF resulted in upregulation of $S O X 9, C O L 2 A 1$ and $A C A N$ (Figure 2A).

In a previous study, we suggested that a high level of WNT signaling induces the chondrogenic differentiating hMSCs to undergo hypertrophic differentiation, and that addition of WNT natural antagonists DKK1 and FRZB prevents the hypertrophy of hMSCs during chondrogenesis [25].

We, therefore, explored the effect of high versus low WNT signaling on the expression of hypertrophic markers. In line with our previous study, a high level of canonical WNT signaling induced by BIO significantly increased the expression of RUNX2, ALPL and COL1OA1. In PKF treated cells, which displayed a low tot non-detectable WNT signal, the expression of these hypertrophic genes was significantly decreased (Figure 2B).

We then examined the effect of canonical WNT signaling on the expression of catabolic genes MMPs, ADAMTS 4/5 and the MMPs inhibitor TIMP3. Interestingly, treatment of BIO significantly decreased the expression of $M M P$ 1/3/9/13, while it increased TIMP3 expression. In addition, BIO treatment increased aggrecanase ADAMTS 4/5 expression. However, MMP 9 increased in the PKF treated group while TIMP3, MMP13 and ADAMTS 4/5 strongly decreased. $M M P 1 / 3$ did not show a significant change (Figure 2C).

The dedifferentiation marker COL1A1 was also measured by qPCR. As Figure 2D shows, BIOinduced high WNT signaling greatly increased the expression of COL1A1, while PKFinhibited WNT signaling dramatically decreased COL1A1 expression. In addition, we also observed that the two key glycolysis genes GULT1 and PGK1 were highly expressed in the BIO group and expression was lower in the PKF group (Figure 2D). 


\section{Control}

A

B

C

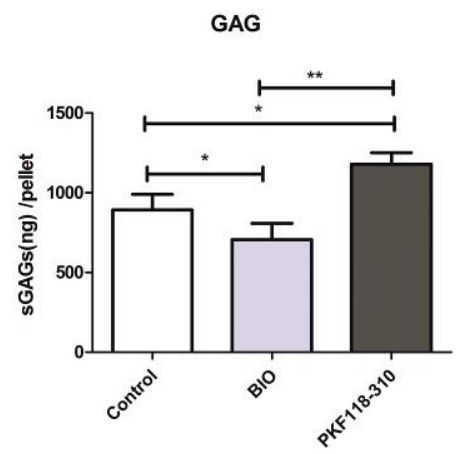

D

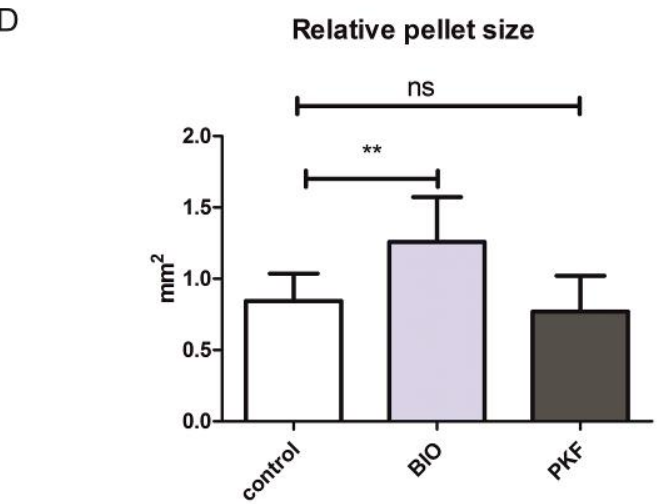

$\mathrm{BIO}$

PKF 118-310
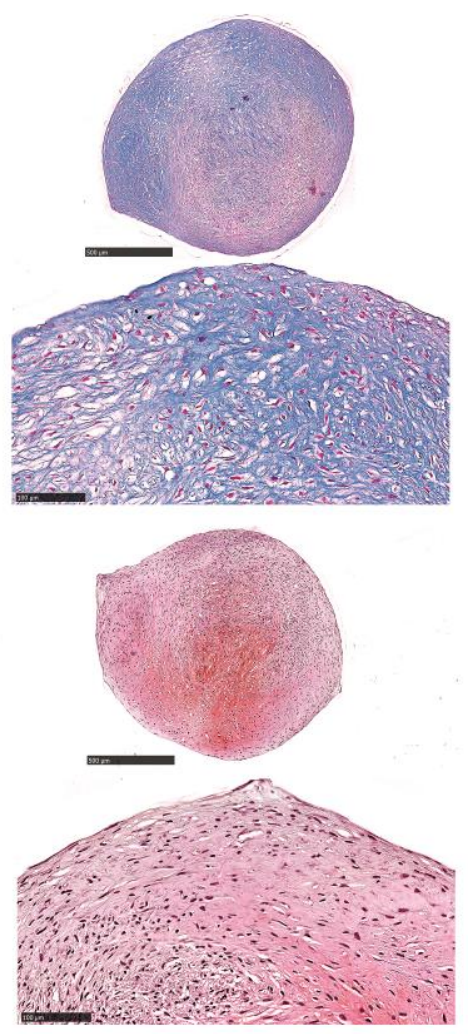

DNA

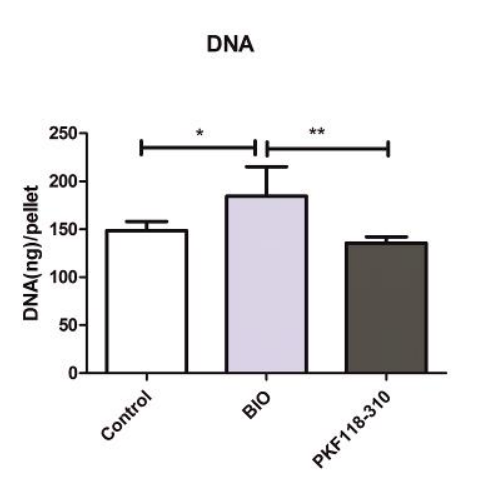

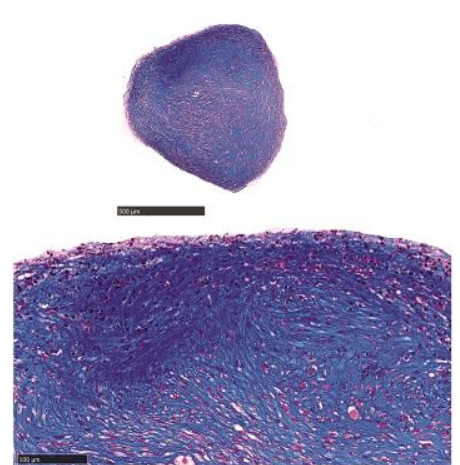
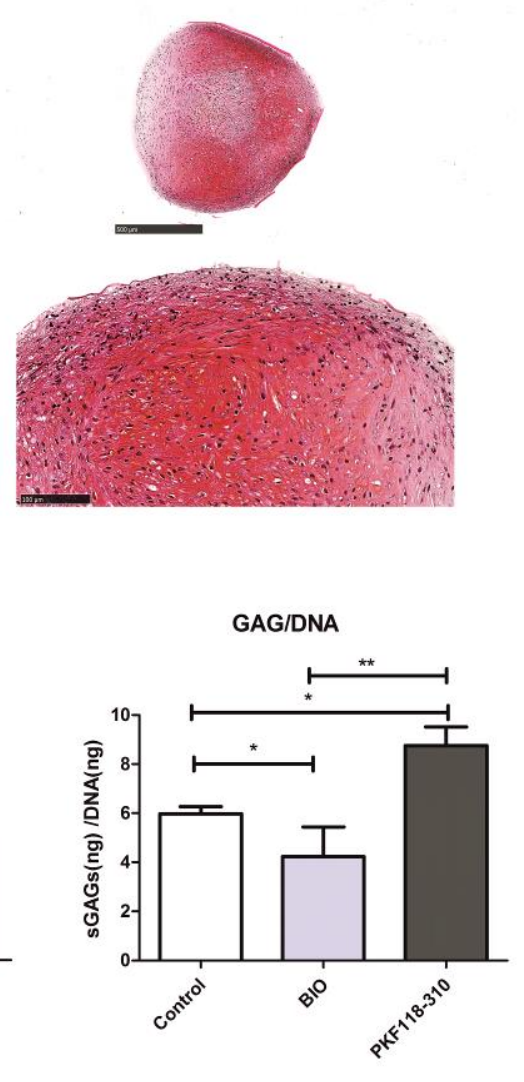

Figure 3 Acitvation of the canonical WNT signaling pathway inhibits cartilage formation. The effects of small molecules on cartilage formation and pellet size after 5 weeks of culture. A. Alcian blue 
staining was performed to examine deposition of sulfated GAGs in midsagittal paraffin sections of hMSCs pellets, nuclei were counterstained with Nuclear fast red. Upper panel indicated the overview of each pellet, scale bar $=500 \mu \mathrm{m}$; lower panel indicated magnified pictures, scale bar $=100 \mu \mathrm{m}$. B. Safranin O staining, nuclei were counterstained with hematoxylin. Upper panel indicated the overview of each pellet, scale bar $=500$; lower panel indicated magnified pictures, scale bar $=100 \mu \mathrm{m} . \mathrm{C}, \mathrm{GAG}$ and DNA production in pellet. D. Quantification of the average size of the pellets. $* \mathrm{P}<0.05, * * \mathrm{P}<0.01$. Error bars represent Standard Deviation (S. D.).

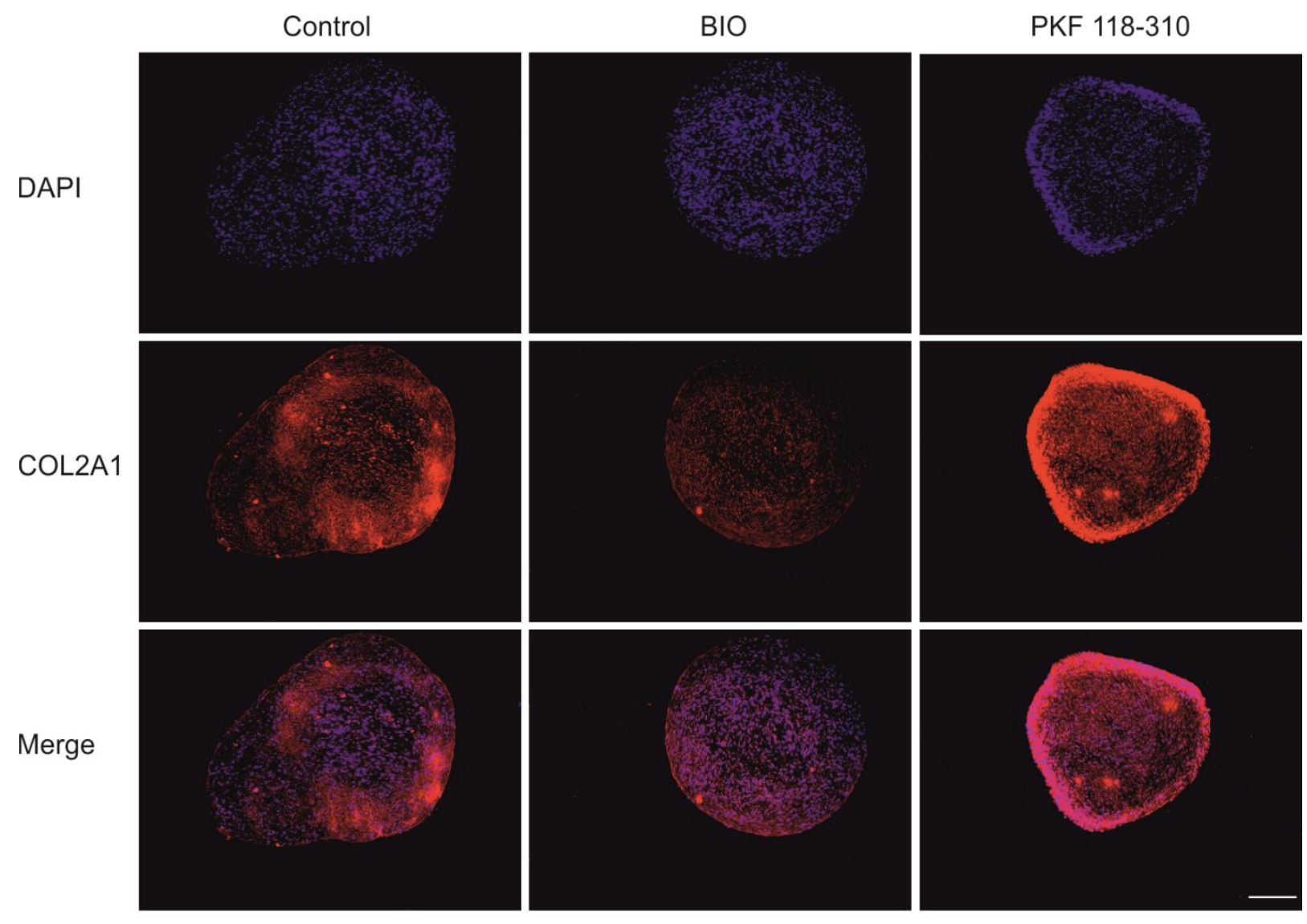

Figure 4 Activation of canonical WNT signaling prevents collagen type 2 production, while inhibition of canonical WNT signaling enhances collagen type 2 production. The expression of type II collagen was visualized by immunofluorescence in midsagittal paraffin sections. Alexa®Fluor 546labelled second antibody was used to detect the positive type II collagen expression. Nucleus was counterstained by DAPI. Scale bar $=500 \mu \mathrm{m}$. 


\section{High levels of canonical WNT signaling inhibited cartilage formation}

To study the contribution of canonical WNT signaling to cartilage matrix formation after 5 weeks of chondrogenesis, histological staining was performed. Alcian blue/Safranin O staining and GAG assay (Figures 3A, 3B and 3C) indicated that $\mathrm{BIO}$ treatment decreased cartilage formation, while PKF increased cartilage formation. Moreover, addition of BIO increased both the total DNA synthesis, and the average diameter of the pellets significantly. There were no significant differences between the DNA content and pellet size in the PKF and control groups (Figures 3C and 3D). In line with GAG staining and the chemical assay, immunofluorescence indicated that BIO slightly downregulated collagen type II expression, while PKF promoted its expression (Figure 4).

\section{BIO significantly decreased the number of apoptotic cells, while PKF induced hMSCs apoptosis in pellets}

It is evident from the qPCR result that BIO significantly induced the expression of hypertrophic markers. We assumed that the treatment of BIO might induce cells towards hypertrophic differentiation. In order to investigate hypertrophic differentiation, the typical hypertrophy marker type X collagen was detected by immunohistochemistry. Type X collagen could hardly be detected in either the control or the PKF group. However, BIO greatly induced the expression of COL10A1 (Figure 5A).

During the process of hypertrophic differentiation, cells will enlarge, terminally differentiate and ultimately undergo mineralization. In order to further elucidate this process, Alizarin red $\mathrm{S}$ staining was performed to evaluate mineralization. No matrix mineralization was observed in any of the groups (Figure 5B).

Several members of the WNT family, such as WNT16, WNT7a and TCF4, have been shown to be involved in the regulation of chondrocyte apoptosis [26-28]. In order to investigate if the alteration of WNT signaling by BIO/PKF will modulate cell apoptosis, a TUNEL assay was used to detect apoptotic cells. Apoptotic cells were highly present in the PKF group but barely present in the BIO treatment, as shown in Figure 5B. Compared to control, the number of apoptotic cells was decreased in the BIO treated group while it had increased in the PKF treated group (Figure 5C). 
A

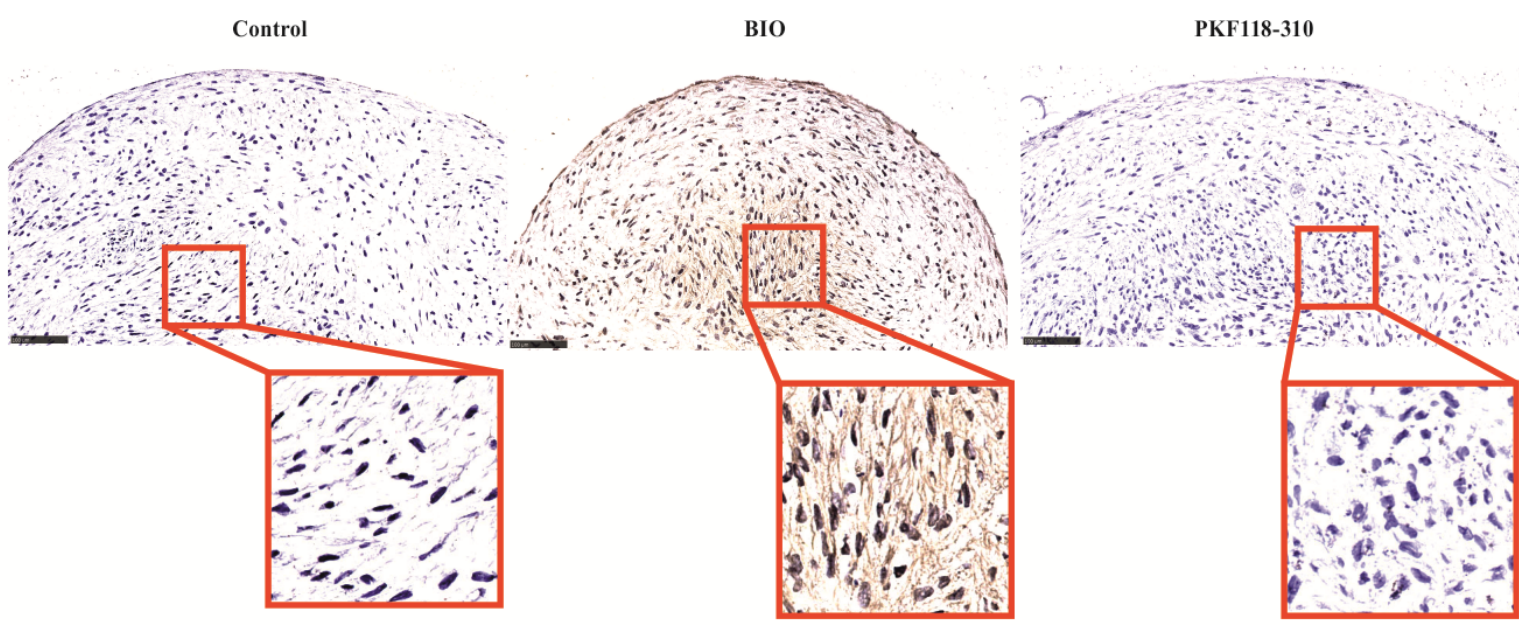

B
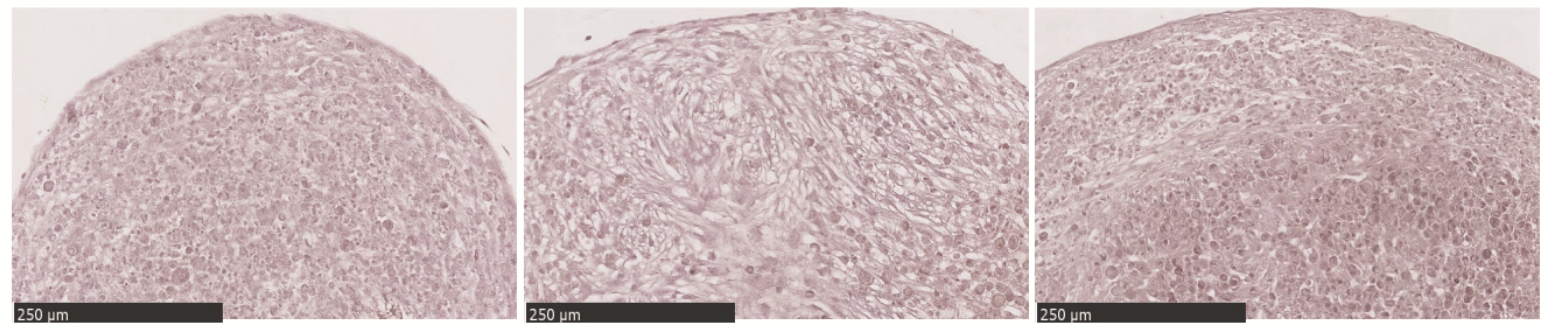

C
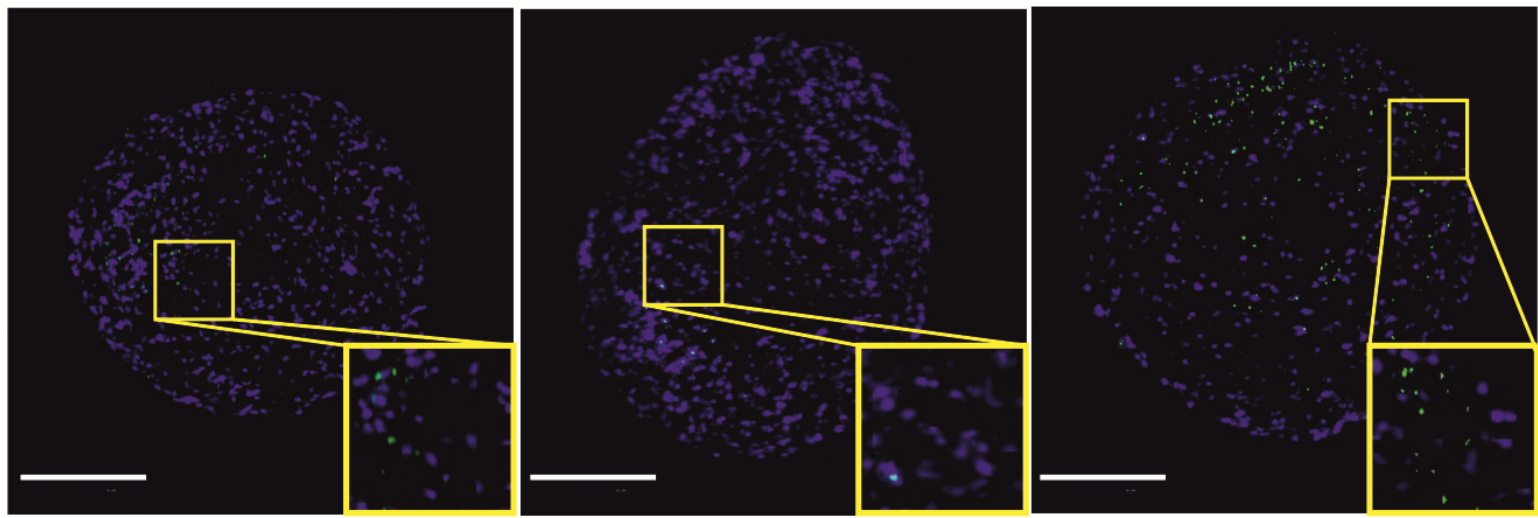

D

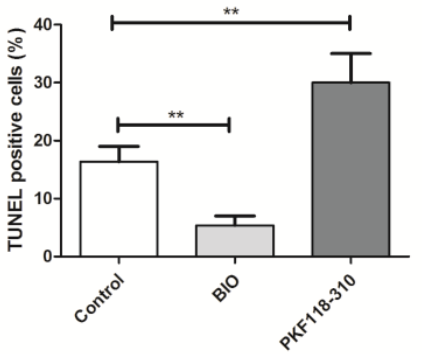

Figure 5 The effects of BIO or PKF on the hypertrophy, mineralization and apoptosis of hMSCs pellets. A. Immunohistochemistry of Collagen type X. The Collagen type X was detected by mouse 
anti-human Collagen type $\mathrm{X}$ antibody. Cell nuclei were counterstained with hematoxylin. Images were taken using a Nanozoomer. Scale bar $=100 \mu \mathrm{m}$. B. Alizarin red staining for matrix mineralization. Scale bar $=250 \mu \mathrm{m}$. C. TUNEL assay. Positive apoptosis cell (green) was stained using the DeadEnd Fluorometric TUNEL System. Nuclei were counterstained with Hoechst 33342 (blue). Scale bar = $500 \mu \mathrm{m}$. D. Quantification of apoptotic cells. $* \mathrm{P}<0.05$, $* * \mathrm{P}<0.01$. Error bars represent Standard Deviation (S. D.).

A

ELISA DKK1

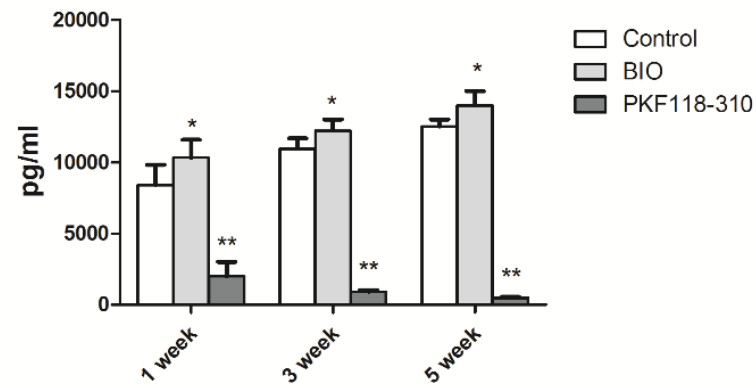

B

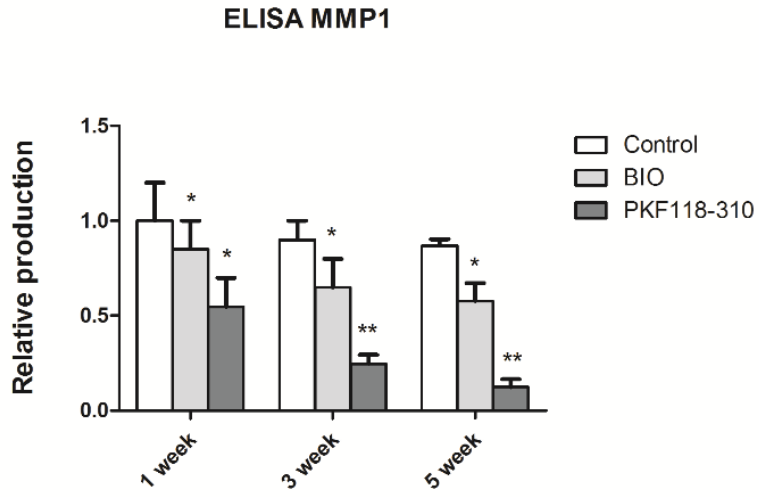

Figure 6 The protein concentration of DKK1 and MMP1 in the pellet culture medium. hMSCs pellets were treated with BIO or PKF for 5 weeks. The medium was collected in week 1, 3 and 5, respectively. The concentrations of DKK1 (A.) and MMP1 (B.) were measured by ELISA. $* \mathrm{P}<0.05$, $* * \mathrm{P}<0.01$. Error bars represent Standard Deviation (S. D.).

\section{The protein level of DKK1 and MMP1 was decreased in pellet culture medium with PKF treatment}

Previously, we found that DKK1, as a natural WNT antagonist, plays a vital role in the regulation of chondrogenic differentiation of hMSCs. In this study, we collected the medium and measured the concentration of endogenous DKK1 secreted from hMSC pellets after treatment with BIO or PKF by ELISA. After continuous stimulation for 5 weeks, BIO slightly increased the expression of DKK1. However, PKF dramatically decreased DKK1 expression to a very low level (Figure 6A). The relative protein level of MMP1 was also measured in the collected medium. The concentration of MMP1 gradually decreased over time in all the treatments. Both BIO and PKF decreased the MMP1 expression. Compared to BIO treatment, the PKF treatment inhibited the expression of MMP1 more effectively (Figure 6B). 


\section{Discussion}

Cell-based cartilage regeneration often involves the use of chondrocytes or hMSCs. However, hMSCs chondrogenic differentiation is still unsatisfactory. An improvement in current cartilage tissue-engineering protocols requires more detailed insight into the molecular mechanisms that regulate the distinct steps of chondrogenic differentiation of hMSCs. We propose that targeting the canonical WNT signaling pathway to increase chondrogenic differentiation of MSCs would be a very important and a valuable strategy.

In the present study, we have attempted to investigate the effect of two small molecules, BIO and PKF, which have been described as positive and negative regulators of the WNT signaling pathway [12-15]. Our objective was to further improve the chondrogenic microenvironment by regulating canonical WNT signaling in chondrogenic differentiation medium. In addition, we aimed to recapitulate the dual role of WNT signaling in chondrogenically differentiating hMSCs.

We found that the addition of the canonical WNT activator BIO downregulated the expression of cartilage-specific genes and increased cartilage matrix degradation. In contrast, addition of the WNT inhibitor PKF upregulated the expression of cartilage markers and increased the cartilage matrix deposition. This is consistent with other findings showing that the expression pattern of three cartilage-specific genes are downregulated in cultures with $1 \mu \mathrm{M}$ BIO treatment in mice MSCs cells, while PKF increased the cartilage markers in mouse fetal metatarsals [21, 22].

The effects of $\mathrm{BIO}$ and $\mathrm{PKF}$ on chondrogenic differentiation are mediated through the canonical WNT signaling pathway, as both factors modulate the WNT pathway via $\beta$-catenin. BIO functions through preventing $\beta$-catenin breakdown through inhibition of GSK3 $\beta$, while PKF prevents the interaction of $\beta$-catenin with transcription factors, TCF/LEF. The observed effects of BIO and PKF are in line with the observations from transgenic animals in which $\beta$ Catenin is modulated $[29,30]$. We therefore conclude that our observations are mainly caused by perturbation of WNT signaling although the contribution of minor off-target effects cannot be excluded.

Mesenchymal stem cells show promising results in cell-based treatment for cartilage regeneration. Unfortunately, more and more studies are suggesting that the loss of the chondrogenic phenotype and expression of hypertrophic markers, including COL10A1, 
MMP13 and VEGF, are observed in chondrogenically differentiated hMSCs [31-33]. This raises problems for the clinical application of MSCs, since hypertrophy could eventually lead to ossification and apoptosis. WNT/ $\beta$-catenin signaling in cartilage plays a dual role. Activity is essential for chondrocyte proliferation and maintenance of the phenotypic characteristics [34], but excessive activity increases chondrocyte hypertrophy and the expression of cartilage degrading matrix metalloproteinases [35]. In this study, we found that PKF inhibited cell hypertrophy by inhibiting hypertrophic markers, while BIO induced expression of hypertrophic markers. This is in line with our previous study, which showed that blocking WNT signaling by its antagonists DKK1 and FRZB decreases hypertrophic differentiation of hMSCs [36]. Similarly, activation of WNT signaling by neutralization of DKK1 and FRZB promotes hypertrophy of chondrogenic differentiating hMSCs and subsequent matrix mineralization, while it decreases the expression of cartilage markers $[25,36]$. In this study, the measured DKK1 concentration by ELISA was extremely low in PKF treatment. Also, two other WNT genes, $A P C$ and $G S K 3 \beta$, were expressed at very low levels(data not shown). These factors could serve as positive feedbacks of modulation for low WNT signaling, as regulated by PKF.

MMPs are the main proteinases degrading collagens and proteoglycans in joint disease. Previous research by our group shows that in human chondrocytes WNT/ $\beta$-catenin is a potent inhibitor of MMP expression in both basic and IL1 $1 \beta$-treated conditions. The results also show that WNT3a decreased the mRNA expression of MMP 1/3/13 in human MSCs [37]. Similar effects of high WNT level on MMPs were observed in this study. More specifically, BIO significantly decreased the expression of MMP1/3/9/13 and increased TIMP3 expression in hMSC. The WNT inhibitor PKF was found to decrease TIMP3 and MMP13, but increased the MMP9 expression. Considering the inhibitory role of TIMP3 on MMPs, it is no surprise that the expression of TIMP3 was negatively correlated with MMP9. Although MMP13 is one of the proteinases, it is also a typical hypertrophic marker. Its expression can be decreased by alternative pathways such as RUNX2 and FGF2 mediated pathways[38]. The MMP1 protein level dramatically decreased in the PKF-treated medium. We cannot explain the inconsistent expression of MMP1 between protein and gene level on the basis of our current knowledge. It may be that alternative pathways are involved in the regulation of MMP1 post-translational modification.

It has been shown that overexpression of $\beta$-catenin, using a $\beta$-catenin activator or in APC knockout mice, leads to the dedifferentiation of chondrocytes $[39,40]$. We found that a high level of WNT signaling induced by BIO significantly increased COLIAl expression, which 
further proves the role of $\mathrm{WNT} / \beta$-catenin signaling in chondrocyte dedifferentiation. In addition, considering the metabolic ATP requirement of MSCs shifts from oxidative phosphorylation to predominantly glycolytic metabolism during chondogenic differentiation [36], two key glucolysis genes GLUT1 and PGK1 were detected in this study. Interestingly, their expression were elevated in BIO group while dramatically decreased in PKF group, which may indicated the glucolysis metabolism in PKF treated cell were inhibited greatly.

Mineralization is the ultimate end stage of chondrocyte hypertrophy. To investigate whether $\mathrm{BIO}$ treated chondrogenically differentiating MSCs also undergo matrix mineralization, Alizarin red S staining was adopted to observe the mineralization nodule. No mineralization was observed in either the control or the experimental group in this study, which suggest that the hypertrophic differentiation did not reach the end stage. In addition, it has been shown that chondrocyte hypertrophy does not have to be related to chondrocyte mineralization [41].

Apoptosis is an energy-dependent and highly orchestrated process in which cells eventually commit suicide without inducing inflammatory injury to the surrounding tissues [42]. One study has shown that inhibition of $\beta$-catenin signaling in articular chondrocytes causes increased cell apoptosis in transgenic mice model [43]. It has also been demonstrated that hMSCs alone or in co-culture with chondrocytes undergo apoptosis during chondrogenesis due to serum withdrawal and prolonged cell culture $[44,45]$. In our study, the apoptosis assay has shown that apoptotic cells were present in both control and experimental groups cultured in medium without serum. Many studies reported BIO as an anti-apoptotic agent that inhibits apoptosis after serum deprivation [46, 47]. We found that BIO significantly decreased the number of apoptotic cells, while PKF induced cell apoptosis.

Activated Wnt $/ \beta$-catenin signaling is well known to stimulate the cell proliferation in multiple cell cultures in vitro $[16-18,48,49]$. We observed that pellets with BIO treatment showed increased diameter and DNA numbers. Considering the anti-apoptotic effects and proliferation stimulating effects of BIO on cultured cells, it is no surprise that the pellets enlarged after BIO treatment.

Taken together, the chondrogenic differentiation of hMSCs, characterized by high expression of chondrocyte markers $S O X 9$, COL2A1 and ACAN, was inhibited by BIO-induced high WNT signaling, while being promoted by the lower WNT activitiy after PKF treatment. Moreover, active canonical WNT signaling induced the expression of chondrocyte hypertrophic markers such as $R U N X 2, C O L 10 A 1$ and $A L P L$, while their expression was reduced by lowering the 
WNT activity (Figure 7). Our findings suggest that fine-tuning canonical WNT signals in the chondrogenic differentiation process of hMSCs could contribute to better cartilage tissue engineering.

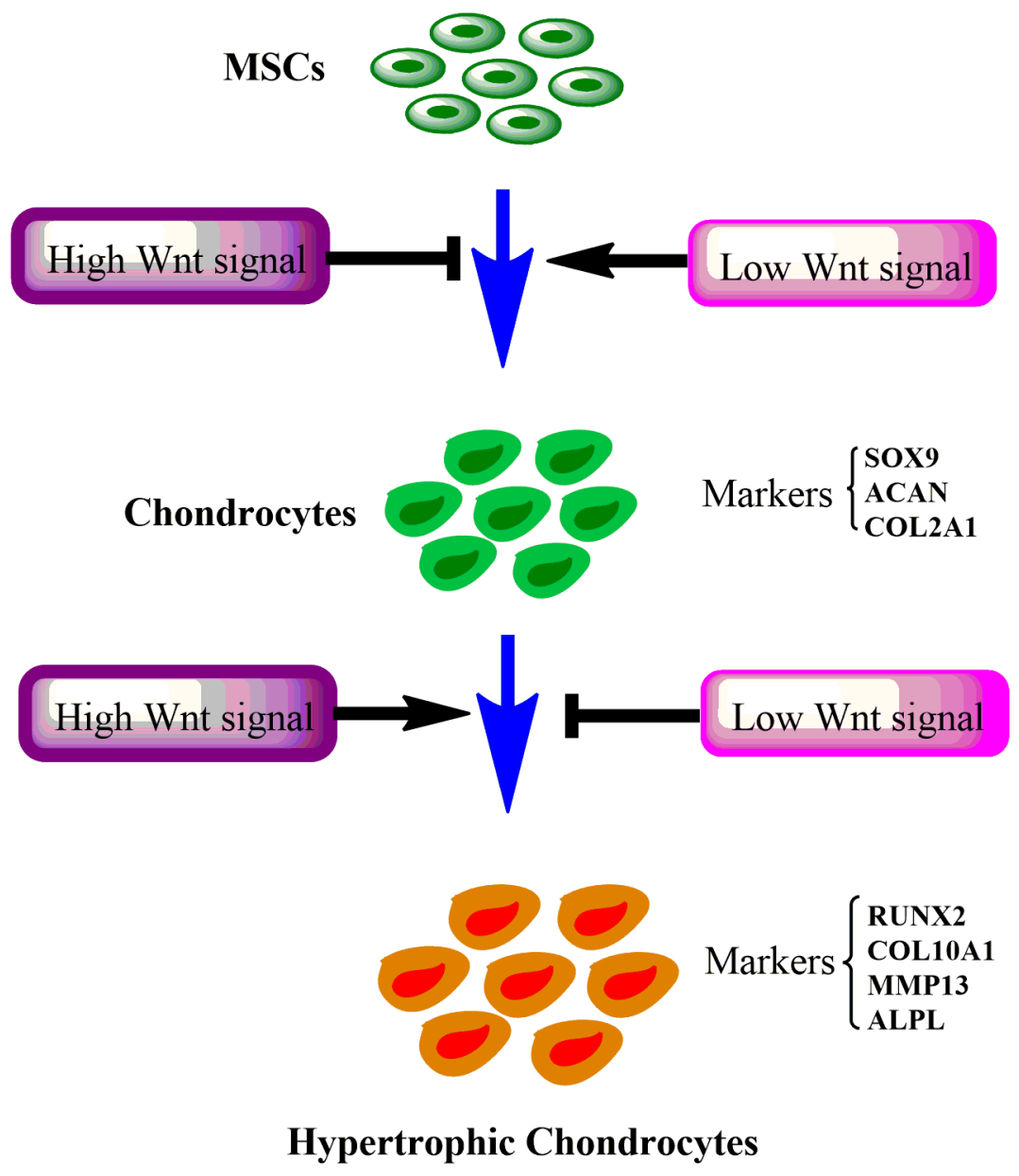

Figure 7 The schematic overview for the chondrogenic differentiation of hMSC regulated by WNT signaling. High levels of WNT signaling inhibits the chondrogenic differentiation of hMSCs evidenced by decreased SOX9, ACAN and COL2A1 expression, but promotes the hypertrophy of chondrocytes exemplified by the induction of expression of RUNX2, COL1OA1, MMP13 and ALPL. In contrast, low levels of WNT signaling promotes hMSC chondrogenic differentiation and inhibits chondrocyte hypertrophy. 


\section{References}

1. Pittenger, M.F., et al., Multilineage potential of adult human mesenchymal stem cells. Science, 1999. 284(5411): p. 143-7.

2. Worster, A.A., et al., Chondrocytic differentiation of mesenchymal stem cells sequentially exposed to transforming growth factor-betal in monolayer and insulinlike growth factor-I in a three-dimensional matrix. J Orthop Res, 2001. 19(4): p. 73849.

3. Noth, U., A.F. Steinert, and R.S. Tuan, Technology insight: adult mesenchymal stem cells for osteoarthritis therapy. Nat Clin Pract Rheumatol, 2008. 4(7): p. 371-80.

4. $\quad$ Miller, J.R., The Wnts. Genome Biol, 2002. 3(1): p. Reviews3001.

5. Logan, C.Y. and R. Nusse, The Wnt signaling pathway in development and disease. Annu Rev Cell Dev Biol, 2004. 20: p. 781-810.

6. Miller, J.R., et al., Mechanism and function of signal transduction by the Wnt/betacatenin and Wnt/Ca2+ pathways. Oncogene, 1999. 18(55): p. 7860-72.

7. Clevers, H., Wnt/beta-catenin signaling in development and disease. Cell, 2006. 127(3): p. $469-80$.

8. Neth, P., et al., Wnt signaling regulates the invasion capacity of human mesenchymal stem cells. Stem Cells, 2006. 24(8): p. 1892-903.

9. Kirton, J.P., et al., Wnt/beta-catenin signaling stimulates chondrogenic and inhibits adipogenic differentiation of pericytes: potential relevance to vascular disease? Circ Res, 2007. 101(6): p. 581-9.

10. Hoppler, S. and C.L. Kavanagh, Wnt signalling: variety at the core. J Cell Sci, 2007. 120(Pt 3): p. 385-93.

11. Tran, F.H. and J.J. Zheng, Modulating the wnt signaling pathway with small molecules. Protein Sci, 2017.

12. Yasuhara, R., et al., Wnt/beta-catenin and retinoic acid receptor signaling pathways interact to regulate chondrocyte function and matrix turnover. J Biol Chem, 2010. 285(1): p. 317-27.

13. Lepourcelet, M., et al., Small-molecule antagonists of the oncogenic Tcf/beta-catenin protein complex. Cancer Cell, 2004. 5(1): p. 91-102.

14. Wei, W., et al., Small molecule antagonists of Tcf4/beta-catenin complex inhibit the growth of HCC cells in vitro and in vivo. Int J Cancer, 2010. 126(10): p. 2426-36. 
15. Gandhirajan, R.K., et al., Small molecule inhibitors of Wnt/beta-catenin/lef-1 signaling induces apoptosis in chronic lymphocytic leukemia cells in vitro and in vivo. Neoplasia, 2010. 12(4): p. 326-35.

16. Kim, W.Y., et al., Essential roles for GSK-3s and GSK-3-primed substrates in neurotrophin-induced and hippocampal axon growth. Neuron, 2006. 52(6): p. 981-96.

17. Cao, H., et al., GSK3 inhibitor-BIO regulates proliferation of immortalized pancreatic mesenchymal stem cells (iPMSCs). PLoS One, 2012. 7(2): p. e31502.

18. $\mathrm{Hu}, \mathrm{Y}$., et al., GSK3 inhibitor-BIO regulates proliferation of female germline stem cells from the postnatal mouse ovary. Cell Prolif, 2012. 45(4): p. 287-98.

19. Tseng, A.S., F.B. Engel, and M.T. Keating, The GSK-3 inhibitor BIO promotes proliferation in mammalian cardiomyocytes. Chem Biol, 2006. 13(9): p. 957-63.

20. Sato, N., et al., Maintenance of pluripotency in human and mouse embryonic stem cells through activation of Wht signaling by a pharmacological GSK-3-specific inhibitor. Nat Med, 2004. 10(1): p. 55-63.

21. Baghaban Eslaminejad, M. and N. Fallah, Small Molecule-BIO Accelerates and Enhances Marrow-Derived Mesenchymal Stem Cell in Vitro Chondrogenesis. Iran J Med Sci, 2014. 39(2): p. 107-16.

22. Landman, E.B., et al., Small molecule inhibitors of WNT/beta-catenin signaling block IL-1beta- and TNFalpha-induced cartilage degradation. Arthritis Res Ther, 2013. 15(4): p. R93.

23. Leow, P.C., et al., Antitumor activity of natural compounds, curcumin and PKF118310, as Wnt/beta-catenin antagonists against human osteosarcoma cells. Invest New Drugs, 2010. 28(6): p. 766-82.

24. Hallett, R.M., et al., Small molecule antagonists of the Wnt/beta-catenin signaling pathway target breast tumor-initiating cells in a Her2/Neu mouse model of breast cancer. PLoS One, 2012. 7(3): p. e33976.

25. Leijten, J.C., et al., Gremlin 1, frizzled-related protein, and Dkk-1 are key regulators of human articular cartilage homeostasis. Arthritis Rheum, 2012. 64(10): p. 3302-12.

26. Hwang, S.G., et al., Wnt-7a causes loss of differentiated phenotype and inhibits apoptosis of articular chondrocytes via different mechanisms. J Biol Chem, 2004. 279(25): p. 26597-604.

27. Ma, B., et al., T cell factor 4 is a pro-catabolic and apoptotic factor in human articular chondrocytes by potentiating nuclear factor kappaB signaling. J Biol Chem, 2013. 288(24): p. 17552-8. 
28. Nalesso, G., et al., WNT16 antagonises excessive canonical WNT activation and protects cartilage in osteoarthritis. Ann Rheum Dis, 2017. 76(1): p. 218-226.

29. Zhu, M., et al., Activation of beta-catenin signaling in articular chondrocytes leads to osteoarthritis-like phenotype in adult beta-catenin conditional activation mice. J Bone Miner Res, 2009. 24(1): p. 12-21.

30. Lories, R.J., et al., Articular cartilage and biomechanical properties of the long bones in Frzb-knockout mice. Arthritis Rheum, 2007. 56(12): p. 4095-103.

31. Freyria, A.M. and F. Mallein-Gerin, Chondrocytes or adult stem cells for cartilage repair: the indisputable role of growth factors. Injury, 2012. 43(3): p. 259-65.

32. Huey, D.J., J.C. Hu, and K.A. Athanasiou, Unlike bone, cartilage regeneration remains elusive. Science, 2012. 338(6109): p. 917-21.

33. Puetzer, J.L., J.N. Petitte, and E.G. Loboa, Comparative review of growth factors for induction of three-dimensional in vitro chondrogenesis in human mesenchymal stem cells isolated from bone marrow and adipose tissue. Tissue Eng Part B Rev, 2010. 16(4): p. $435-44$.

34. Yasuhara, R., et al., Roles of beta-catenin signaling in phenotypic expression and proliferation of articular cartilage superficial zone cells. Lab Invest, 2011. 91(12): p. 1739-52.

35. Blom, A.B., et al., Involvement of the Wnt signaling pathway in experimental and human osteoarthritis: prominent role of Wnt-induced signaling protein 1. Arthritis Rheum, 2009. 60(2): p. 501-12.

36. Zhong, L., et al., Endogenous DKK1 and FRZB Regulate Chondrogenesis and Hypertrophy in Three-Dimensional Cultures of Human Chondrocytes and Human Mesenchymal Stem Cells. Stem Cells Dev, 2016. 25(23): p. 1808-1817.

37. Ma, B., C.A. van Blitterswijk, and M. Karperien, A Wnt/beta-catenin negative feedback loop inhibits interleukin-1-induced matrix metalloproteinase expression in human articular chondrocytes. Arthritis Rheum, 2012. 64(8): p. 2589-600.

38. Wang, X., et al., Regulation of MMP-13 expression by RUNX2 and FGF2 in osteoarthritic cartilage. Osteoarthritis Cartilage, 2004. 12(12): p. 963-73.

39. Ryu, J.H., et al., Regulation of the chondrocyte phenotype by beta-catenin. Development, 2002. 129(23): p. 5541-50.

40. Miclea, R.L., et al., Adenomatous polyposis coli-mediated control of beta-catenin is essential for both chondrogenic and osteogenic differentiation of skeletal precursors. BMC Dev Biol, 2009. 9: p. 26. 
41. van der Kraan, P.M. and W.B. van den Berg, Chondrocyte hypertrophy and osteoarthritis: role in initiation and progression of cartilage degeneration? Osteoarthritis Cartilage, 2012. 20(3): p. 223-32.

42. Sinha, D., et al., Lithium activates the Wnt and phosphatidylinositol 3-kinase Akt signaling pathways to promote cell survival in the absence of soluble survival factors. Am J Physiol Renal Physiol, 2005. 288(4): p. F703-13.

43. Zhu, M., et al., Inhibition of beta-catenin signaling in articular chondrocytes results in articular cartilage destruction. Arthritis Rheum, 2008. 58(7): p. 2053-64.

44. Alvarez-Tejado, M., et al., Hypoxia induces the activation of the phosphatidylinositol 3-kinase/Akt cell survival pathway in PC12 cells: protective role in apoptosis. J Biol Chem, 2001. 276(25): p. 22368-74.

45. Wu, L., et al., Trophic effects of mesenchymal stem cells increase chondrocyte proliferation and matrix formation. Tissue Eng Part A, 2011. 17(9-10): p. 1425-36.

46. Bonegio, R. and W. Lieberthal, Role of apoptosis in the pathogenesis of acute renal failure. Curr Opin Nephrol Hypertens, 2002. 11(3): p. 301-8.

47. Cross, D.A., et al., Selective small-molecule inhibitors of glycogen synthase kinase-3 activity protect primary neurones from death. J Neurochem, 2001. 77(1): p. 94-102.

48. Lee, H.C., S. Lim, and J.Y. Han, Wnt/beta-catenin signaling pathway activation is required for proliferation of chicken primordial germ cells in vitro. Sci Rep, 2016. 6: p. 34510 .

49. Hoffman, M.D. and D.S. Benoit, Agonism of Wnt-beta-catenin signalling promotes mesenchymal stem cell (MSC) expansion. J Tissue Eng Regen Med, 2015. 9(11): p. E13-26. 


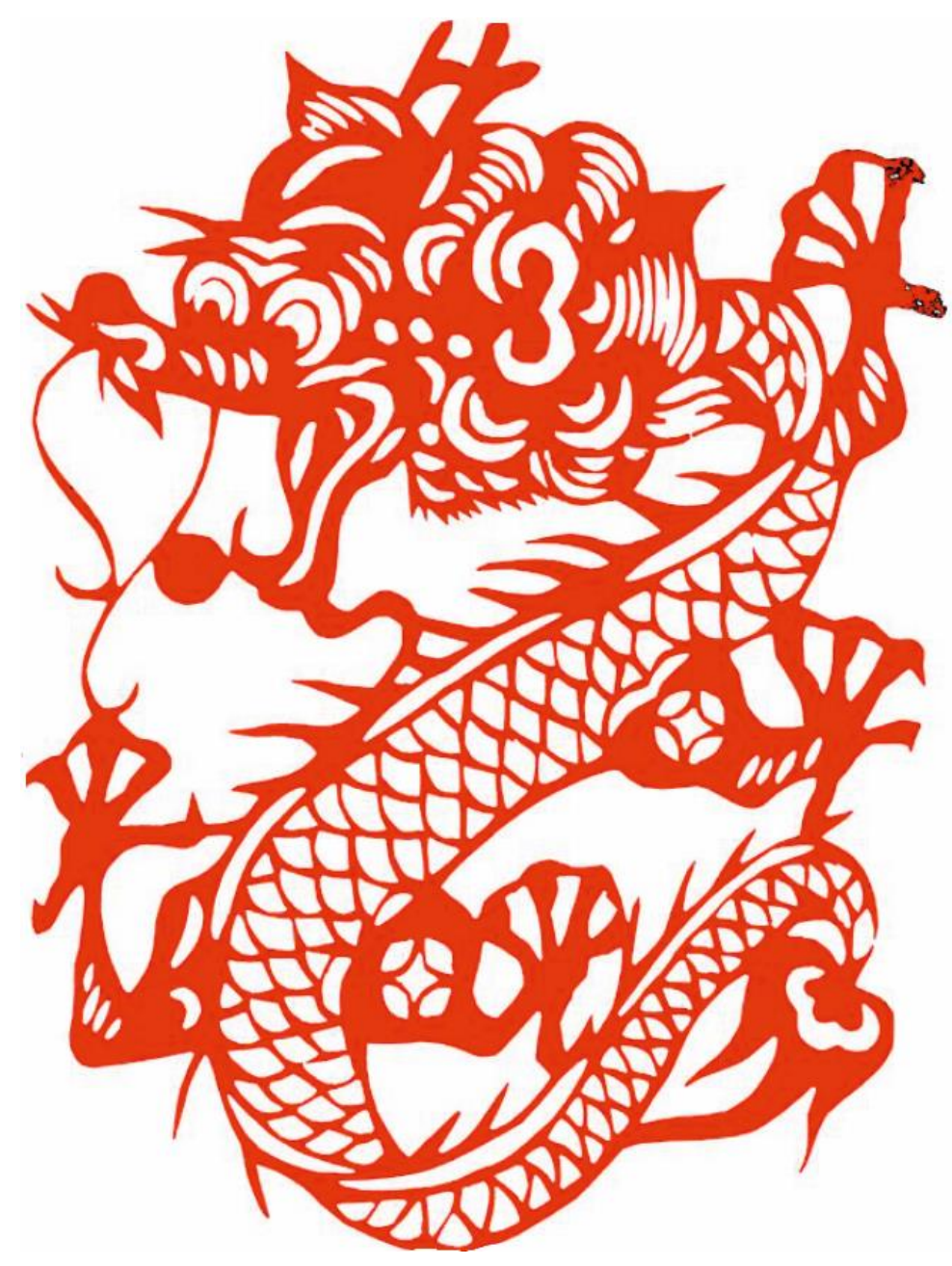




\section{Chapter 5}

\section{Hypoxia greatly promotes the redifferentiation of human chondrocytes and diminishes the difference between health and osteoarthritic chondrocytes}

Xiaobin Huang, Leilei Zhong, Jan Hendriks, Janine N. Post and Marcel Karperien

Developmental BioEngineering, MIRA Institute for Biomedical Technology and Technical

Medicine, University of Twente, Enschede 7500 AE, The Netherlands

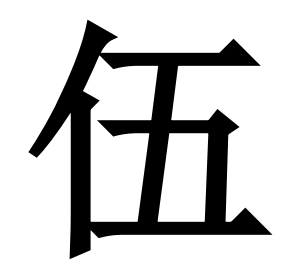

上善若水，水善利万物而不争。处众人之所恶，故几于道。

The highest good is like that of water. The goodness of water is that it benefits the ten thousand creatures; yet itself does not scramble, but is content with the places that all men disdain. It is that makes water so near to the Way. 


\begin{abstract}
Objective: Due to its avascular nature, articular cartilage is relatively hypoxic. In contrast to most experiments where chondrocytes are cultured in an atmospheric oxygen concentration, which can be considered non-physiological, we used a hypoxic condition for chondrocyte culture. We hypothesized that oxygen tension critically influences the responses of healthy or diseased articular chondrocytes to extracellular stimuli like pro-inflammatory cytokines. The aim of this study is to determine how oxygen tension influences the response of healthy and osteoarthritic chondrocytes to IL1 $\beta$.
\end{abstract}

Methods: 2-D expanded macroscopically healthy looking (HL) and osteoarthritic (OA) chondrocytes were redifferentiated in three-dimensional (3-D) pellet cultures in chondrogenic differentiation medium, supplemented with or without IL1 $\beta$ at $21 \% \mathrm{O}_{2}$ (normoxia) or $2.5 \% \mathrm{O}_{2}$ (hypoxia) for 3 weeks. Alcian blue/Safranin O and GAG assay were used to measure cartilage matrix production. The expression of chondrocyte markers, hypertrophic markers and catabolic genes were measured by real-time quantitative PCR (RT-PCR). The protein expression of collagen type II, HIF $1 \alpha$ and HIF $2 \alpha$ were detected by immunofluorescence. Cell proliferation and apoptosis were assessed by EdU staining and TUNEL assay respectively. ELISA was used to measure the protein level of MMP1 in the pellet-cultured medium.

Results: Hypoxia enhanced matrix production in both HL and OA chondrocytes and this effect was stronger on OA chondrocytes. Under hypoxia, both HL and OA chondrocytes had a higher expression of cartilage markers $S O X 9$ and $A C A N$, and a lower expression of catabolic genes $M M P 1 / 3 / 13$ and the dedifferentiation marker COL1A1. Under normoxia, HL chondrocytes showed higher expression of cartilage markers and lower catabolic genes expression than OA chondrocytes. Interestingly, hypoxia diminished the difference between HL and OA chondrocytes. Under IL1 $\beta$ treatment, the gene expression of MMP1/3/9, ADAMTS5 and iNOS was dramatically induced, and both HL and OA cells went into apoptosis in both normoxia and hypoxia. Moreover, cell proliferation was inhibited. In hypoxia, the basic expression levels of the MMPs was much lower in both HL and OA cells compared to cells cultured under normoxia; IL1 $\beta$ potently induced MMPs expression regardless of cell population and oxygen tension. The fold induction of these MMPs was however much higher than in normoxia. HL chondrocytes had higher HIF1 $\alpha$ expression and lower HIF2 $\alpha$ expression than OA chondrocytes 
in normoxia. Hypoxia promoted the expression of HIF1 $\alpha$ and HIF2 $\alpha$ in HL chondrocytes, while it only enhanced HIF $1 \alpha$ expression but decreased the HIF $2 \alpha$ expression in OA chondrocytes.

Conclusions: Hypoxia stimulated the redifferentiation of cultured chondrocytes, particularly in OA chondrocytes derived from macroscopically diseased cartilage. Interestingly, hypoxia diminished the diference between HL and OA chondrocyte which is clearly present in normoxia. The basal levels in MMP expression in both OA and HL chondrocytes is much higher in normoxia suggesting that culture of chondrocytes in normoxia itself is sufficient to induce a mild inflammatory response in chondrocytes. Finally, oxygen tension profoundly and differentially affected the expression of HIF $1 \alpha$ and HIF2 $\alpha$ in both HL and OA chondrocytes suggesting that they may play a vital role in chondrocyte redifferentiation.

Key words: Cartilage; Osteoarthritis; Chondrocyte; hypoxia; IL1 $\beta, \operatorname{HIF} 1 \alpha$; HIF2 $\alpha$ 


\section{Introduction}

Chondrocytes are often used as the cell sources in cartilage engineering for the repair of damaged cartilage, as they are the only cell type in cartilage. Due to the limitation of cell quantity, the use of chondrocytes for cartilage repair requires an in vitro expansion phase to generate the quantity required for therapy $[1,2]$. However, the expansion process in monolayer often leads to dedifferentiation with higher expression of collagen type I (COL1A1), and lower expression of cartilage matrix genes such as aggrecan (ACAN) and collagen type II (COL2A1) [3-7]. The loss of chondrocyte phenotype upon proliferation in vitro was documented as early as in 1960 [8]. This has been a bottleneck for cartilage tissue engineering, since dedifferentiated chondrocytes become fibroblastic and do not produce hyaline cartilage, which is necessary for the proper functioning of articulating joints. Therefore, the redifferentiation of dedifferentiated chondrocytes is a prerequisite in chondrocyte-based cartilage repair. Expanded chondrocytes are commonly redifferentiated through three-dimensional (3-D) pellet cultures in the presence of Transforming Growth Factor $\beta$ (TGF $\beta$ ), dexamethasone (DEX) and other induction factors $[9,10]$. Although 3-D culture was used to achieve the redifferentiation of chondrocytes, hypertrophy markers were also upregulated $[11,12]$. Thus, it is urgent to acquire more details and find more clues to promote the redifferentiation and inhibit the hypertrophy of chondrocytes for restoration of cartilage in joint diseases, such as osteoarthritis (OA).

Healthy articular cartilage is a typical avascular tissue. Articular chondrocytes are embedded in an extensive extracellular matrix and exposed to a concentration of approximately $6 \% \mathrm{O}_{2}$ in the superficial layer, to a concentration as low as less than $1 \% \mathrm{O}_{2}$ in the calcified layer, depending on the zone in the cartilage $[13,14]$. Chondrocytes have been shown to be well adapted to the low-oxygen conditions and capable of maintaining their energy metabolism, mainly through the glycolytic pathway [15]. Hypoxia is considered to be a positive influence on the healthy chondrocyte phenotype and cartilage matrix formation $[16,17]$. The hypoxic response is mainly mediated by hypoxia inducible factors (HIF), which includes three family members, HIF-1 $\alpha,-2 \alpha,-3 \alpha$ [18]. HIF- $1 \alpha$ and HIF-2 $\alpha$ in particular play an active role in chondrocyte development. HIF-1a, is reported to promote chondrogenesis of MSCs [19], in part by activating SOX9 [20]. The chondrogenic and anti-catabolic effect of hypoxia in pellet culture of human articular chondrocytes is blocked by a HIF-1 $\alpha$ inhibitor [21]. HIF-2 $\alpha$ is reported to enhance promoter activities of COL10A1, MMP13, and VEGFA through specific binding to the respective hypoxia-responsive elements, and is essential for endochondral 
ossification of cultured chondrocytes and embryonic skeletal growth in mice [22]. In addition, HIF-2 $\alpha$ causes cartilage matrix destruction by upregulating crucial catabolic genes, such as MMPs, ADAMTS4, nitric oxide synthase-2 (NOS2), and prostaglandin endoperoxide synthase2 (PTGS2) [23]. Furthermore, it potentiates FAS-mediated chondrocyte apoptosis [24]. The balance between HIF-1 $\alpha /$ HIF-2 $\alpha$ activities could contribute to cartilage homeostasis. Conventional cell culture experiments with chondrocytes are performed at an atmospheric oxygen concentration, which can be considered as a non-physiological, hyperoxic condition for chondrocytes.

Chondrocytes in native cartilage are exposed to a variety of biochemical and genetic factors [25], and OA chondrocytes are often exposed to an abnormal environment, such as highmagnitude mechanical stress, inflammatory cytokines, or altered amounts or organization of matrix proteins, including degradation products [26]. Inflammation has been implicated in the pathogenesis of OA [27], and pro-inflammatory cytokines, such as Interleukin-1 (IL1 $\beta$ ), are potently involved in a degenerative process by inducing MMP expression and cartilage degradation in vitro and in vivo [28-30]. Besides its role in cartilage degradation by stimulating MMPs, IL1 $\beta$ also blocks the synthesis of new extracellular matrix components to impair the ability of the cartilage to restore the extracellular matrix [31].

Therefore, chondrocytes normally face a hypoxic environment, combined in a pathophysiological situation with an inflammatory environment in vivo. In this study, we investigated whether the responsiveness of chondrocytes to IL1 $\beta$ is depending on oxygen tension. In addition, we also compared the response of healthy and OA chondrocytes in these conditions.

\section{Materials and methods}

\section{Cell culture and expansion}

Institutional approval to isolate primary human cells was obtained. Healthy (HL) and osteoarthritic (OA) primary human chondrocytes were, respectively, isolated from macroscopically healthy looking areas and damaged regions of femoral condyles in four patients (mean \pm SD age $60 \pm 3$ years) undergoing total knee replacement, as described previously [32]. To isolate cells, the cartilage was digested in chondrocyte proliferation 
medium containing $0.15 \%$ collagenase type II (Worthington) for 20-22 hours (hrs). After extensive washing, the human chondrocytes were subsequently expanded at a density of 3000 cells $/ \mathrm{cm}^{2}$ in chondrocyte proliferation medium until the monolayer reached $80 \%$ confluency. Chondrocyte proliferation medium consisted of DMEM supplemented with $10 \%$ fetal bovine serum (FBS), $1 \times$ non-essential amino acids, $0.2 \mathrm{mM}$ ascorbic acid 2-phosphate (AsAP), 0.4 $\mathrm{mM}$ proline, $100 \mathrm{U} / \mathrm{mL}$ penicillin and $100 \mu \mathrm{g} / \mathrm{ml}$ streptomycin.

\section{Pellet cultures and chondrocytes redifferentiation}

To form high-density cell pellets, 250,000 cells were seeded per well in a round bottom 96wells plate in chondrogenic differentiation medium (DMEM supplemented with $50 \mu \mathrm{g} / \mathrm{mL}$ ITS-premix, $50 \mu \mathrm{g} / \mathrm{mL}$ AsAP, $100 \mu \mathrm{g} / \mathrm{mL}$ sodium pyruvate, $10-7 \mathrm{M}$ dexamethasone, $10 \mathrm{ng} / \mathrm{mL}$ TGF- $\beta 3,100 \mathrm{U} / \mathrm{mL}$ penicillin and $100 \mu \mathrm{g} / \mathrm{ml}$ streptomycin) and centrifuged for $3 \mathrm{~min}$ at 2000rpm, as previously described [33]. The pellets were cultured with or without IL1 $\beta$ (Biolegend) stimulation in 10ng/ml for 3 weeks at $37{ }^{\circ} \mathrm{C}, 5 \% \mathrm{CO} 2,21 \% \mathrm{O} 2$ (for normoxia) and $2.5 \% \mathrm{O} 2$ (for hypoxia). Passage 3 chondrocytes were used in this research. The medium was refreshed every three days.

\section{Total RNA extraction and quantitative polymerase chain reaction (qPCR)}

Cell pellets were crashed by grinding rod and RNA was isolated using Trizol reagent (Thermo Fisher Scientific). The concentration and purity of RNA samples were determined using a Nanodrop 2000 (Thermo scientific). Total mRNA was reverse-transcribed into cDNA using the iScript cDNA Synthesis kit (Bio-Rad). qPCR was performed using the SYBR Green sensimix (Bioline). PCR reactions were carried out using a Bio-Rad CFX96 (Bio-Rad) under the following conditions: cDNA was denatured for $5 \mathrm{~min}$ at $95{ }^{\circ} \mathrm{C}$, followed by 39 cycles consisting of $15 \mathrm{~s}$ at $95^{\circ} \mathrm{C}, 15 \mathrm{~s}$ at $60^{\circ} \mathrm{C}$ and $30 \mathrm{~s}$ at $72{ }^{\circ} \mathrm{C}$. For each reaction a melting curve was generated to test for primer dimer formation and non-specific priming. Gene expression was normalized using RPL13A and expressed as fold induction compared to controls.

\section{Alcian blue and Safranin O staining}

Pellets were fixed with $10 \%$ phosphate-buffered formalin $(\mathrm{pH}=7)$ for $2 \mathrm{hrs}$ at room temperature (RT), dehydrated with graded ethanols and embedded in paraffin, using routine procedures. Sections of $5 \mu \mathrm{m}$ thickness were cut using a microtome (Shandon). Before staining, the slides were deparaffinized in xylene and rehydrated with graded ethanols. The slides were either stained for sulfated glycosaminoglycans (GAG) with a $0.5 \% \mathrm{w} / \mathrm{v}$ solution of Alcian blue 
$(\mathrm{pH}=1$, adjusted with $\mathrm{HCl}$ ) for $30 \mathrm{~min}$, or stained for sulfated $\mathrm{GAG}$ with a $0.1 \%$ solution of Safranin O for 5 min (Sigma Aldrich). The samples were then counterstained with nuclear fast red $(0.1 \% \mathrm{w} / \mathrm{v}$ of nuclear fast red in $5 \%$ aluminum sulfate) for $5 \mathrm{~min}$ or haematoxylin (Sigma Aldrich) for $1 \mathrm{~min}$ respectively to visualize nuclei. Images were taken using a Nanozoomer (Iwata City, Japan).

\section{Immunofluorescent staining}

Immunofluorescent staining of collagen type II, HIF $1 \alpha$ and HIF $2 \alpha$ were performed on $5 \mu \mathrm{m}$ sections from pellets. Slides were deparaffinized in xylene and rehydrated with graded ethanols. Samples were pre-incubated with $5 \mu \mathrm{g} / \mathrm{mL}$ proteinase K (Sigma Aldrich) for $10 \mathrm{~min}$ at RT followed by $1 \mathrm{mg} / \mathrm{mL}$ hyaluronidase (Sigma Aldrich) for $40 \mathrm{~min}$ at $37{ }^{\circ} \mathrm{C}$. Samples were blocked in 5\% BSA in PBS for 1 hour, then incubated overnight with rabbit anti-human collagen II antibody (ab34712, Abcam), rabbit anti-HIF1 $\alpha$ antibody (Santa Cruz Biotechnology) and mouse anti- HIF2 $\alpha$ antibody ( Santa Cruz Biotechnology) respectively, in a dilution of 1:100 in 5\% BSA in PBS at $4^{\circ}$. Then Alexa ${ }^{\circledR F l u o r ~ 546-l a b e l l e d ~ g o a t ~ a n t i-r a b b i t ~ o r ~ a n t i-m o u s e ~}$ antibody in 5\% BSA in PBS was added and incubated for 2 hours at RT. Samples were rinsed with PBS between every step. Mounting medium with DAPI was added and images were viewed by BD pathway confocal microscopy.

\section{EdU and TUNEL staining}

For labeling newly synthesized DNA, EdU (5-ethynyl-2'-deoxyuridine) was added to the culture medium at a concentration of $10 \mu \mathrm{M}$, and left for 24 hours before harvesting the samples. Cell pellets were then washed with PBS and fixed with $10 \%$ formalin for $15 \mathrm{~min}$. Samples were embedded in cryomatrix, and cut into $7 \mu \mathrm{M}$ sections with a cryotome (Shandon). Sections were permeabilized and stained for EdU with Click-iT® EdU Imaging Kit (ThermoFisher scientific). Cryosections were also stained for DNA fragments with DeadEnd Fluorometric TUNEL System (Promega). Nuclei were counterstained with Hoechst 33342. The images were viewed by means of BD pathway confocal microscopy .

\section{GAG and DNA assay}

After redifferentiation, pellets were digested in $250 \mu 1$ Tris- $\mathrm{HCl}$ buffer $(0.05 \mathrm{M}$ Tris, $1 \mathrm{mM}$ $\mathrm{CaCl} 2, \mathrm{pH} 8.0$ ) with $1 \mathrm{mg} / \mathrm{ml}$ proteinase $\mathrm{K}$ (Roche) for $16 \mathrm{hrs}$. at $56{ }^{\circ} \mathrm{C}$. Diluted samples $(25 \mu \mathrm{l})$ were mixed with $150 \mu$ 1.9-dimethylmethylene blue (DMMB)-dye solution (3.04 g/l glycine, $2.38 \mathrm{~g} / \mathrm{l} \mathrm{NaCl}, 20 \mathrm{mg} / \mathrm{l} \mathrm{DMMB}$ in distilled water) and absorbance was measured at $525 \mathrm{~nm}$. 
The relative cell number was determined by quantification of the total DNA using a QuantiFluor ${ }^{\circledR}$ dsDNA System kit( Promega), according to the manufacturer's instructions.

\section{Enzyme- linked immunosorbent assay (ELISA) and NO production assay}

The culture medium was collected every three days. The secreted MMP1 in medium was measured by ELISA using mouse anti-human MMP1 antibody (MAB901-SP, R\&D systems), followed by incubation with a rabbit anti-mouse antibody coupled to a horse radish peroxidase (HRP). The amount of HRP was developed by adding Tetramethylbenzidine (TMB, 1-Step Ultra TMB-ELISA, Thermo Scientific). The reactions were stopped by adding $\mathrm{H}_{2} \mathrm{SO} 4$, and measured at $450 \mathrm{~nm}$ (Micro Plate Reader). Cell supernatant was also used to quantify the nitrite, using a Griess reaction as described [34].

\section{Statistical analysis}

For the experiments using primary human HL and OA chondrocytes, samples were obtained from four donors. Each experiment was performed in triplicate. Statistical differences between two groups were analyzed by two-tailed student's t-tests or one-way ANOVA. P $<0.05$ was considered statistically significant and indicated with an asterisk. Data is expressed as the mean $\pm \mathrm{SD}$.

\section{Results}

Hypoxia promotes matrix production and reduces the differences between HL and OA chondrocytes

Relative healthy (HL) and OA chondrocytes expanded in monolayer were cultured in micro mass pellets under either normoxia $\left(21 \% \mathrm{O}_{2}\right)$ or hypoxia $\left(2.5 \% \mathrm{O}_{2}\right)$ conditions for 3 weeks to induce chondrocyte redifferentiation. Alcian blue and Safranin O were used to stain the GAG deposition, and chemical GAG assay and DNA assay were performed to measure the concentration of GAG production and the DNA quantity. Both Alcian blue and Safranin O staining showed that HL chondrocytes produced more GAGs than OA chondrocytes in both normoxia and hypoxia, and hypoxia greatly enhanced the GAG production. Interestingly, the increased GAG deposition in hypoxia was more obvious in OA than in healthy chondrocytes. In normoxia, GAG production was significantly decreased in OA chondrocytes compared to HL. However, this difference between HL and OA in GAG production was almost indistinguishable under hypoxia (Figures 1A and 1B). 

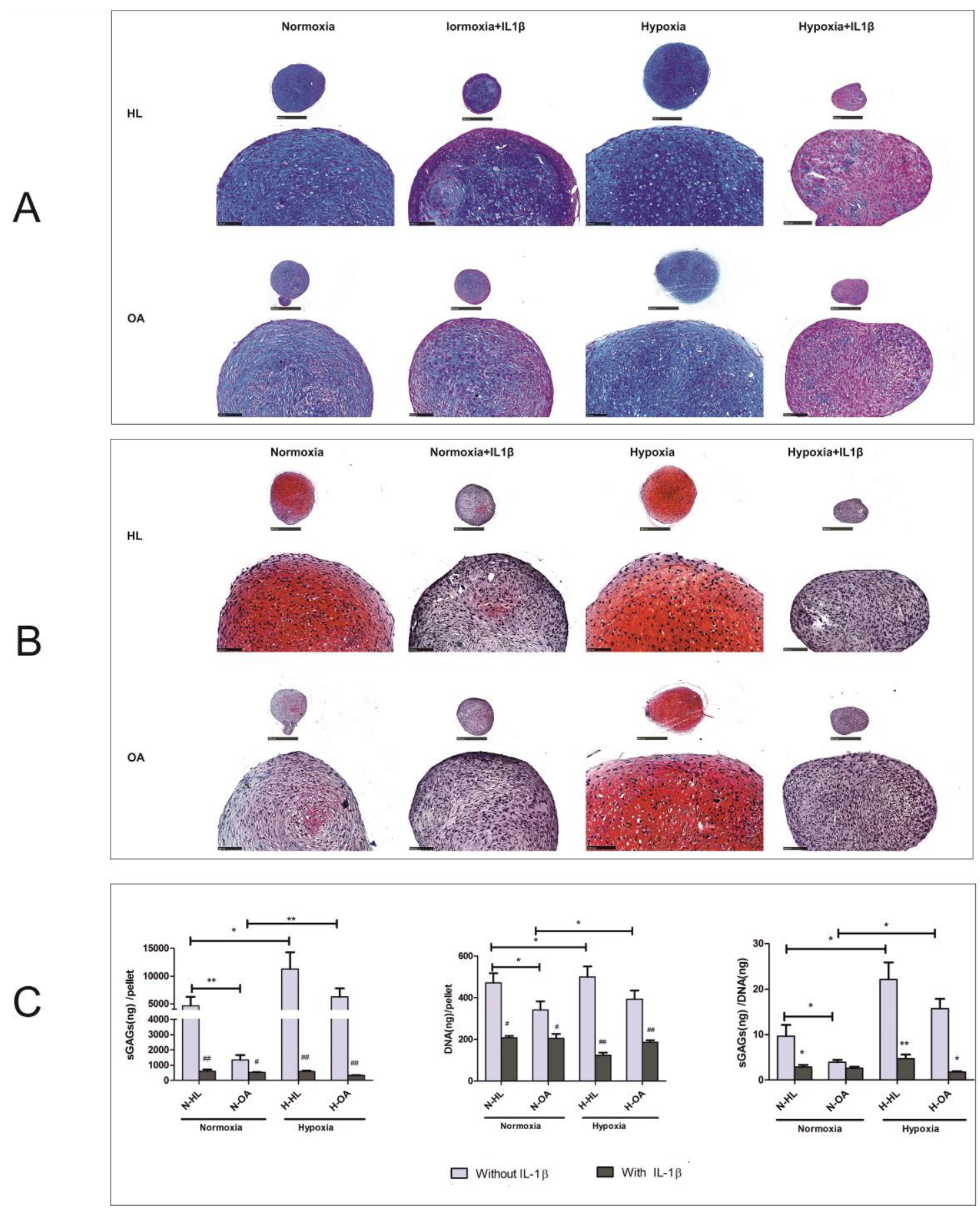

Figure 1 GAG production and collagen type II deposition.A. Alcian blue staining for GAG presence. Nuclei were counterstained by nuclear fast red. HL, healthy chondrocyte; OA, osteoarthritic chondrocyte. Upper panel shows overview of pellets, scale bar $=500 \mu \mathrm{m}$, while lower panel indicates magnified pictures, scale bar $=100 \mu \mathrm{m}$. B. Safranin O staining for GAG presence. Nuclei were counterstained by hematoxylin. Upper panel shows overview of pellets, scale bar $=500 \mu \mathrm{m}$, while lower 
panel indicates magnified pictures, scale bar $=100 \mu \mathrm{m}$. C. GAG and DNA assay. Amount of GAG and DNA in pellets was measured 3 weeks after culture in chondrogenic differentiation medium. N-HL, healthy chondrocyte in normoxia; N-OA, OA chondrocyte in normoxia; H-HL, healthy chondrocyte in hypoxia; H-OA, OA chondrocyte in hypoxia. \# represents the significant difference between IL1 $\beta$ stimulation and corresponding control $(\mathrm{p}<0.05)$; double \# represents $\mathrm{p}<0.01$; * represents $\mathrm{p}<0.05$; double * represents $p<0.01$. Error bar reflects Standard Deviation.

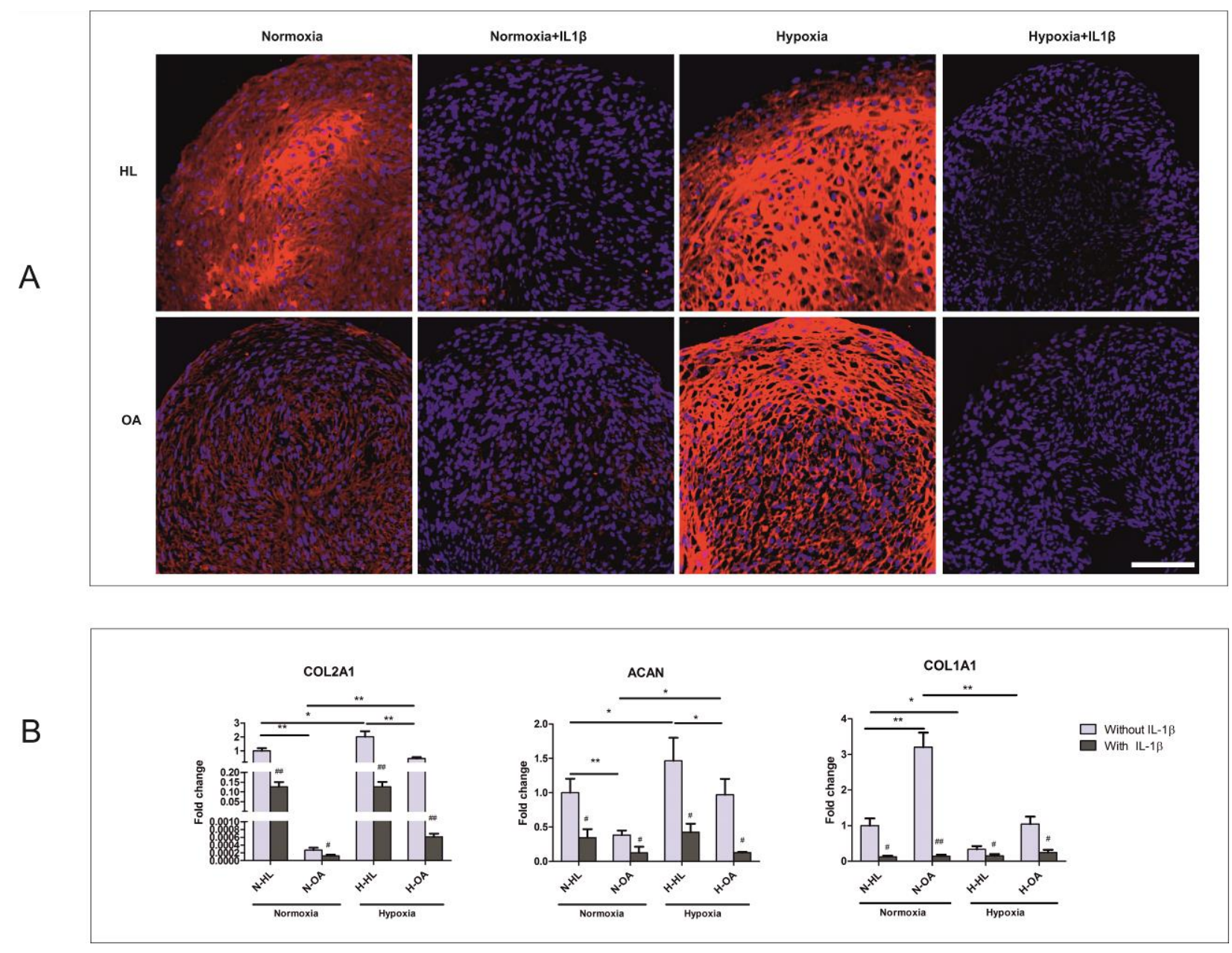

Figure 2 Expression of cartilage matrix genes in hypoxia and normoxia. A. COL2A1 production was detected by mouse anti-COL2A1 antibody (red). Cell nuclei were counterstained with DAPI (blue). Images were taken by BD pathway confocal microscopy. Scare bar $=200 \mu \mathrm{m}$. HL, healthy chondrocyte; OA, osteoarthritic chondrocyte. B. Gene expression of COL2A1, ACAN and COL1A1 was measured by RT-PCR. N-HL, healthy chondrocyte in normoxia; N-OA, OA chondrocyte in normoxia; H-HL, healthy chondrocyte in hypoxia; H-OA, OA chondrocyte in hypoxia. \# represents the significant difference 
between IL1 $\beta$ stimulation and corresponding control $(\mathrm{p}<0.05)$; double \# represents $\mathrm{p}<0.01$; * represents $\mathrm{p}<0.05$; double * represents $\mathrm{p}<0.01$. Error bar reflects Standard Deviation.

The results of GAG quantification were consistent with those of Alcian blue and Safranin O staining (Figure 1C). HL chondrocytes have a higher total GAG content than OA, and GAG deposition in both HL and OA chondrocytes is increased in hypoxia. In particular, hypoxia increased total GAG in OA chondrocytes up to 5 times compared to normoxia, while healthy chondrocytes showed a 2-fold increase. When total GAG content was normalized to DNA, a similar result was found. Next to enhanced GAG production in hypoxia; the amount of DNA was also increased in both HL and OA chondrocytes in hypoxia.

\section{Hypoxia promotes chondrogenic gene expression}

We next evaluated the effect of oxygen tension on the expression of chondrogenic genes. The deposition of COL2A1 was detected by immunofluorescence, and the gene expression of COL2A1, ACAN and COL1A1 was measured by RT-PCR. As Figure 2A shows, hypoxia dramatically increased COL2A1 production in both HL and OA chondrocytes. Interestingly, almost the same amount of COL2A1 was observed in OA chondrocytes compared to HL chondrocytes under hypoxia. This is in line with the Alcian blue/Safranin O staining and GAG assay (Figure 1).

The gene expression level of cartilage markers like COL2AI and ACAN showed the same trend as the histological staining and GAG assay (Figure 2B). In normoxia, HL chondrocytes expressed much more $C O L 2 A 1$ and $A C A N$ than OA chondrocytes, and the expression level in both HL and OA chondrocytes was elevated in hypoxia. Hypoxia induced a 2-fold increase in COL2A1 expression in HL chondrocytes and a more than 1000-fold increase in OA chondrocytes. HL chondrocytes expressed a very low level of the chondrocyte dedifferentiation marker collagen type I (COL1A1). Its expression was strongly increased in OA chondrocyte compared to HL under normoxia. Differentiation in hypoxia reduced the expression of COL1A1 in both HL and OA chondrocytes (Figure 2B).

\section{Hypoxia greatly inhibits the expression of catabolic and hypertrophic marker genes}

The expression of catabolic genes, such as MMP1, MMP3, MMP9, ADAMTS4 and ADAMTS5 and the hypertrophic marker genes MMP13 and COL10A1, was higher in OA chondrocytes than in HL chondrocytes in both normoxia and hypoxia (Figures 3A and 3C). Interestingly, hypoxia significantly decreased the basal levels of expression of $M M P 1, M M P 3, M M P 9$, 
MMP13 and COL1OA1 in both HL and OA chondrocytes. The effect of hypoxia tended to be greater in OA chondrocytes than in HL chondrocytes. There was no difference in the expression of ADAMTS4 and ADAMTS5 in HL chondrocytes between normoxia and hypoxia, whilst the expression of these two genes was significantly inhibited in OA chondrocytes by hypoxia (Figure 3A).

The protein level of MMP1 was measured by ELISA in the pellet culture medium. As Figure 3B shows, the expression of MMP1 gradually decreased over time in both HL and OA chondrocytes under normoxia and hypoxia. Much higher MMP1 expression was observed in OA chondrocytes than in HL chondrocytes under normoxia, while hypoxia significantly decreased MMP1 expression in both HL and OA chondrocytes. In addition, hypoxia greatly minimized the difference between OA and HL chondrocytes.

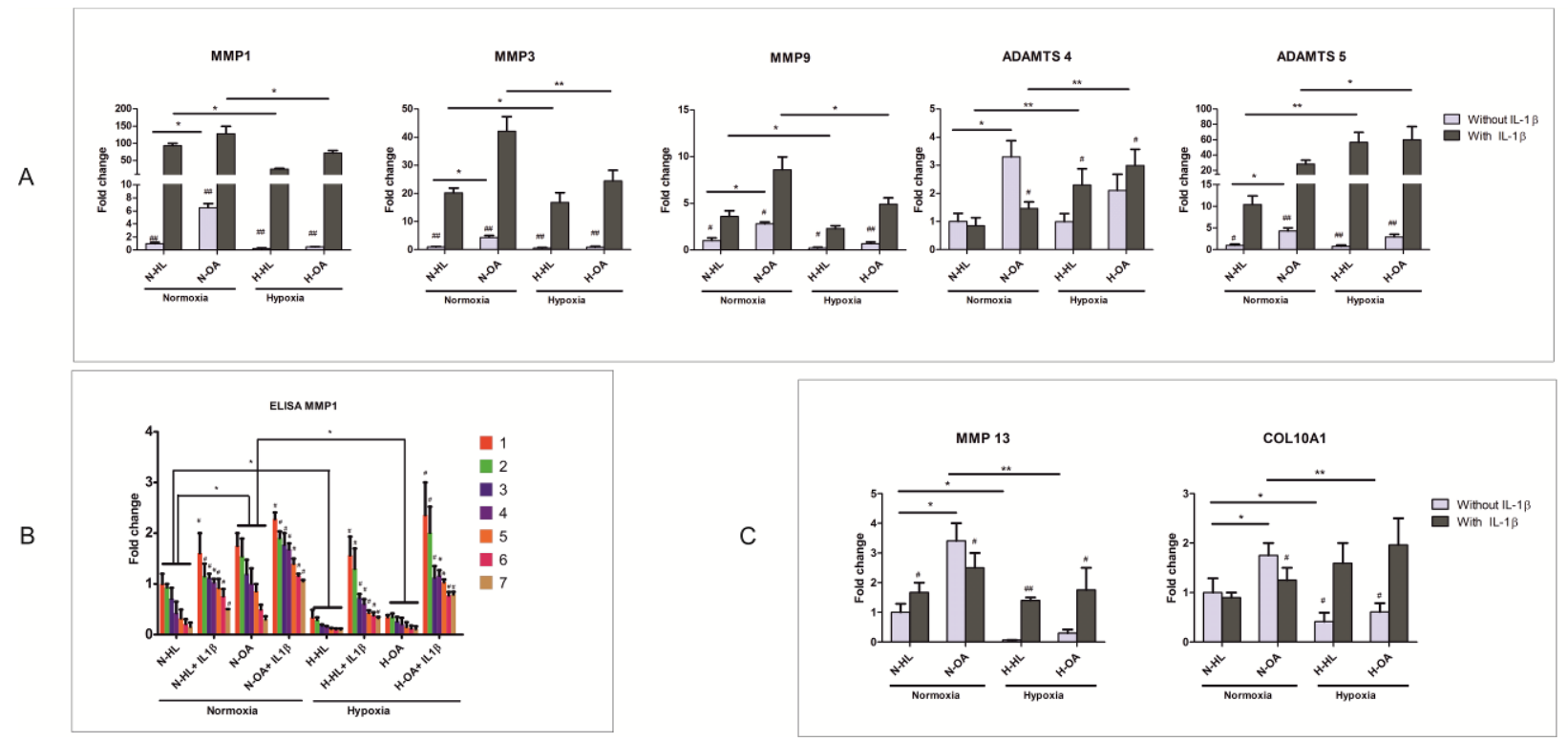

Figure 3 The expression of catabolic and hypertrophic markers in hypoxia and normoxia. A. qPCR was performed to measure the expression of catabolic genes MMP1, MMP3, MMP9, ADAMTS4 and ADAMTS5. B. The amount of MMP1 secreted in medium was measured by ELISA. 1 to 7 represent the order of medium changes every 3 days. C. The expression of hypertrophic markers MMP13, COL1OAl as determined by RT-PCR. N-HL, healthy chondrocyte in normoxia; N-OA, OA chondrocyte in normoxia; H-HL, healthy chondrocyte in hypoxia; H-OA, OA chondrocyte in hypoxia. \# represents the significant difference between IL1 $\beta$ stimulation and corresponding control $(\mathrm{p}<0.05)$; double \# 
represents $\mathrm{p}<0.01 ; *$ represents $\mathrm{p}<0.05$; double * represents $\mathrm{p}<0.01$. Error bar reflects Standard Deviation.

\section{Hypoxia downregulates IL1 $\beta$ induced MMPs expression but fails to reverse IL1 $\beta$-induced matrix loss}

Alcian blue/ Safranin O staining indicated that differentiation of cells in the presence of IL1 $\beta$ dramatically downregulates the GAG production in both HL and OA chondrocytes under normoxia and hypoxia (Figures 1A and 1B). The concentrations of GAG were similar, around 500 ng per pellet, in all conditions with IL1 $\beta$ stimulation. The GAG amount showed an 8-fold decrease in HL and a 3-fold decrease in OA chondrocytes with IL1 $\beta$ treatment under normoxia, but an almost 20-fold decrease in both HL and OA chondrocytes under hypoxia, compared to the corresponding condition without IL1 $\beta$ stimulation (Figure 1C). Likewise, the protein expression of COL2A1 was very low, and was almost undetectable in any of the IL1 $\beta$ stimulated groups in both HL and OA chondrocytes, under both normoxia and hypoxia (Figure $2 \mathrm{~A})$. The gene expression of $C O L 2 A 1$ and $A C A N$ was dramatically decreased in all IL $1 \beta$ treated groups. However, the fold change is much higher in HL than in OA in normoxia in comparison with the hypoxia condition. (Figure 2B). Interestingly, the expression of the dedifferentiation marker COL1A1 was decreased in IL1 $\beta$ treated groups, especially in normoxia. (Figure 2B).

As expected, the expression of IL1 $\beta$ target genes $M M P 1, M M P 3$ and $M M P 9$ was dramatically increased after IL1 $\beta$ stimulation in both normoxia and hypoxia. Although the fold change of MMPs expression induced by IL1 $\beta$ was higher in hypoxia, the overall expression of IL1 $\beta$ induced MMPs was decreased in hypoxia (Figure 3A). Interestingly, IL1 $\beta$ decreased the ADAMTS4 expression in OA chondrocytes compared to HL chondrocytes under normoxia, but increased the expression under hypoxia in both HL and OA chondrocytes. Under IL1 $\beta$ stimulation, the expression of ADAMTS5 was increased in both HL and OA chondrocytes; hypoxia even enhanced this effect (Figure 3A). IL1 $\beta$-induced MMP1 expression in all treated group was also observed in medium by ELISA. MMP1 expression decreased over time in both the IL1 $\beta$ treated and untreated group in either HL or OA chondrocytes under normoxia and hypoxia. In line with gene expression, IL1 $\beta$ induced fold change in MMP1 expression was higher in hypoxia than in normoxia in both HL and OA chondrocytes (Figure 3B). Without IL1 $\beta$, the expression of hypertrophic markers MMP13 and COL1OA1 was decreased in both $\mathrm{HL}$ and OA in hypoxia. Interestingly, under IL1 $\beta$ stimulation the gene expression of MMP13 
and COL1OAl was decreased in OA chondrocytes under normoxia, but greatly increased by IL1 $\beta$ in hypoxia.

A

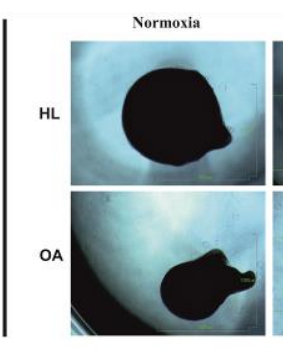

C

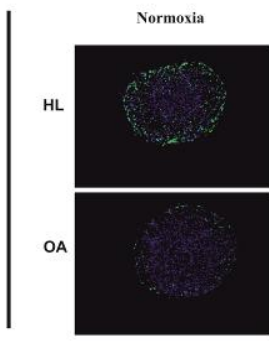

$\mathbf{E}$

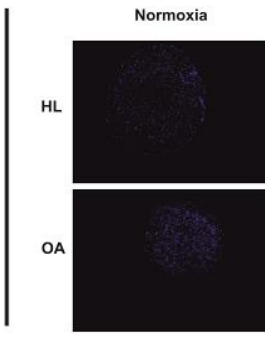

G

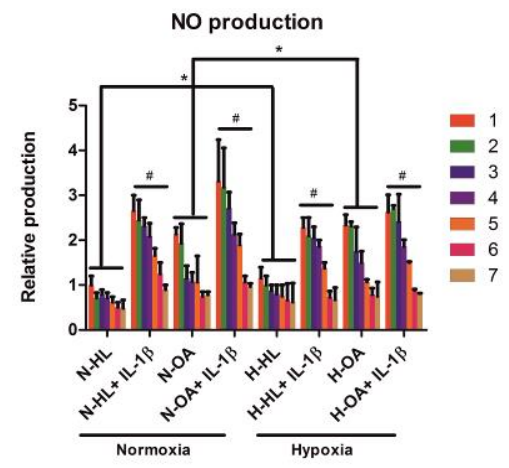

B

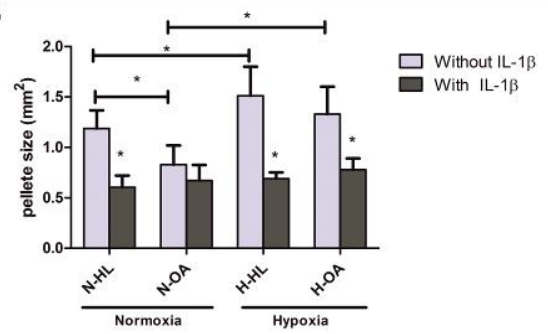

D

Hypoxia+IL1ß
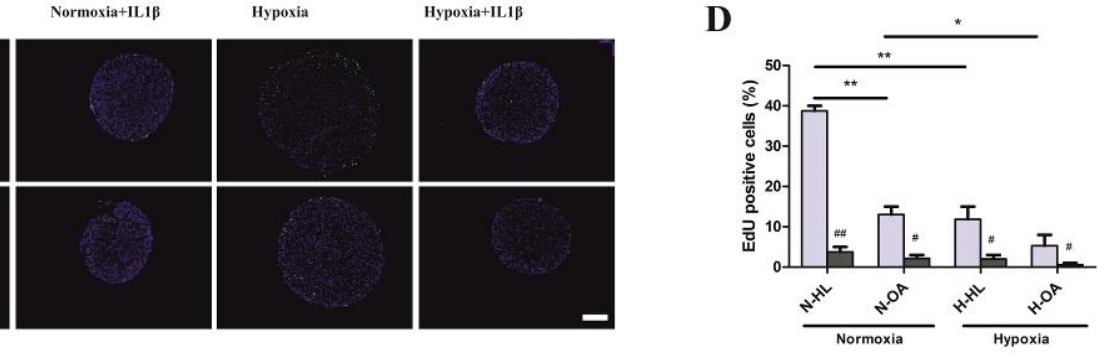

F

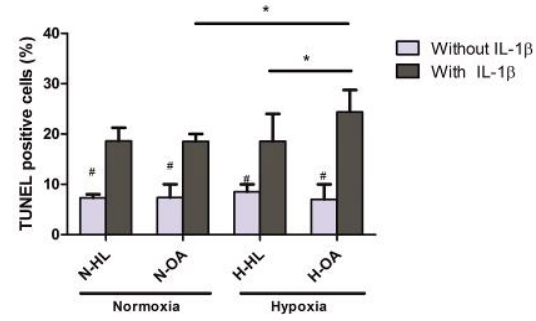

H

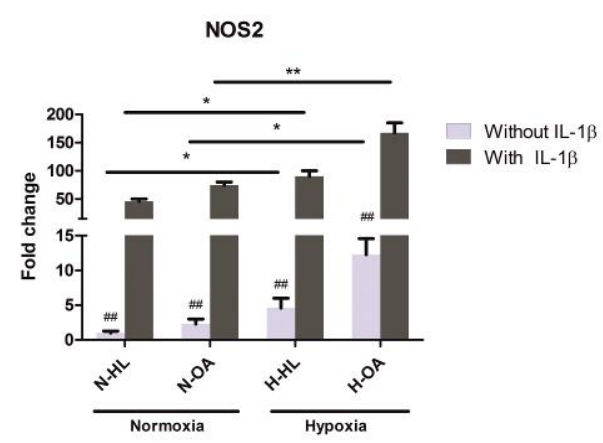

Figure 4 The pellet size, EdU/ TUNEL staining and NO production after IL1ß stimulation. A. Pellets were imaged by light microscopy. Scare bar $=1000 \mu \mathrm{m}$. B. Measurement of pellet size. C. EdU staining of pellets. EdU incorporation into newly synthesized DNA was visualized by Alexa 488 (green). Nuclei were counterstained with Hoechst 33342 (blue). Scale bar $=250 \mu \mathrm{m}$.(D) Quantification of EdU positive chondrocytes. E. TUNEL staining of pellets. TUNEL positive cells were visualized with 
fluorescent labeling (green). Nuclei were counterstained with Hoechst 33342 (blue). Scale bar $=250 \mu \mathrm{m}$. (F) Quantification of TUNEL staining positive cells. G. NO production was measured by Griess reaction. 1 to7 represent the order of medium changes every 3 days. H. The gene expression of iNOS. $\mathrm{N}-\mathrm{HL}$, healthy chondrocyte in normoxia; N-OA, OA chondrocyte in normoxia; H-HL, healthy chondrocyte in hypoxia; H-OA, OA chondrocyte in hypoxia.\# represents the significant difference between IL1 $\beta$ stimulation and corresponding control $(p<0.05)$; double \# represents $p<0.01$; * represents $\mathrm{p}<0.05$; double * represents $\mathrm{p}<0.01$. Error bar reflects Standard Deviation (S. D.).

\section{IL1ß inhibits cell proliferation while inducing cell apoptosis and NO production}

IL1 $\beta$ significantly reduced the pellet size in the HL and OA chondrocyte groups in both normoxia and hypoxia. The pellet size in all IL1 $\beta$ treated groups showed no difference (Figures 4A and 4B). EdU staining indicated more EdU positive cells in HL chondrocytes than in OA chondrocytes. The number of EdU positive cells was decreased in hypoxia. IL1 $\beta$ almost completely inhibited cell proliferation in all treated groups (Figures 4C and 4D). In contrast, IL1 $\beta$ greatly induced cell apoptosis in both HL and OA chondrocytes under normoxia and hypoxia. It was observed that the apoptotic cells, in particular in HL chondrocytes, were mainly present in the periphery of the pellets in normoxia, but were scattered inside the pellets under hypoxia. In hypoxia, OA pellets showed more apoptotic cells than HL pellets, and the amount of apoptotic cells in OA chondrocytes was higher in hypoxia than in normoxia (Figures 4E and $4 \mathrm{~F})$.

IL1 $\beta$ potently induced NO production in HL and OA chondrocytes under both normoxia and hypoxia. NO production in the medium gradually decreased over time in all groups. Interestingly, NO production in HL and OA chondrocytes was slightly induced by hypoxia. However, IL1 $\beta$-induced NO was inhibited by hypoxia (Figure 4G). As expected, iNOS was significantly induced by IL1 $\beta$ in the order of OA $>$ HL. In line with NO production, hypoxia slightly induced iNOS expression in both HL and OA without IL1 $\beta$. However, unlike NO production, hypoxia further elevated IL1 $\beta$-induced iNOS expression compared to normoxia (Figure 4H).

\section{IL1ß greatly enhances the expression of hypoxia-inducible factors (HIF) during chondrocyte redifferentiation}

The protein and gene expression level of hypoxia-inducible factors HIF $1 \alpha$ and HIF $2 \alpha$ were assessed by immunofluorescence and qPCR respectively. As Figure 5A shows, HIF1 $\alpha$ was endogenously expressed in HL chondrocyte and translocated into the nucleus, while it was 

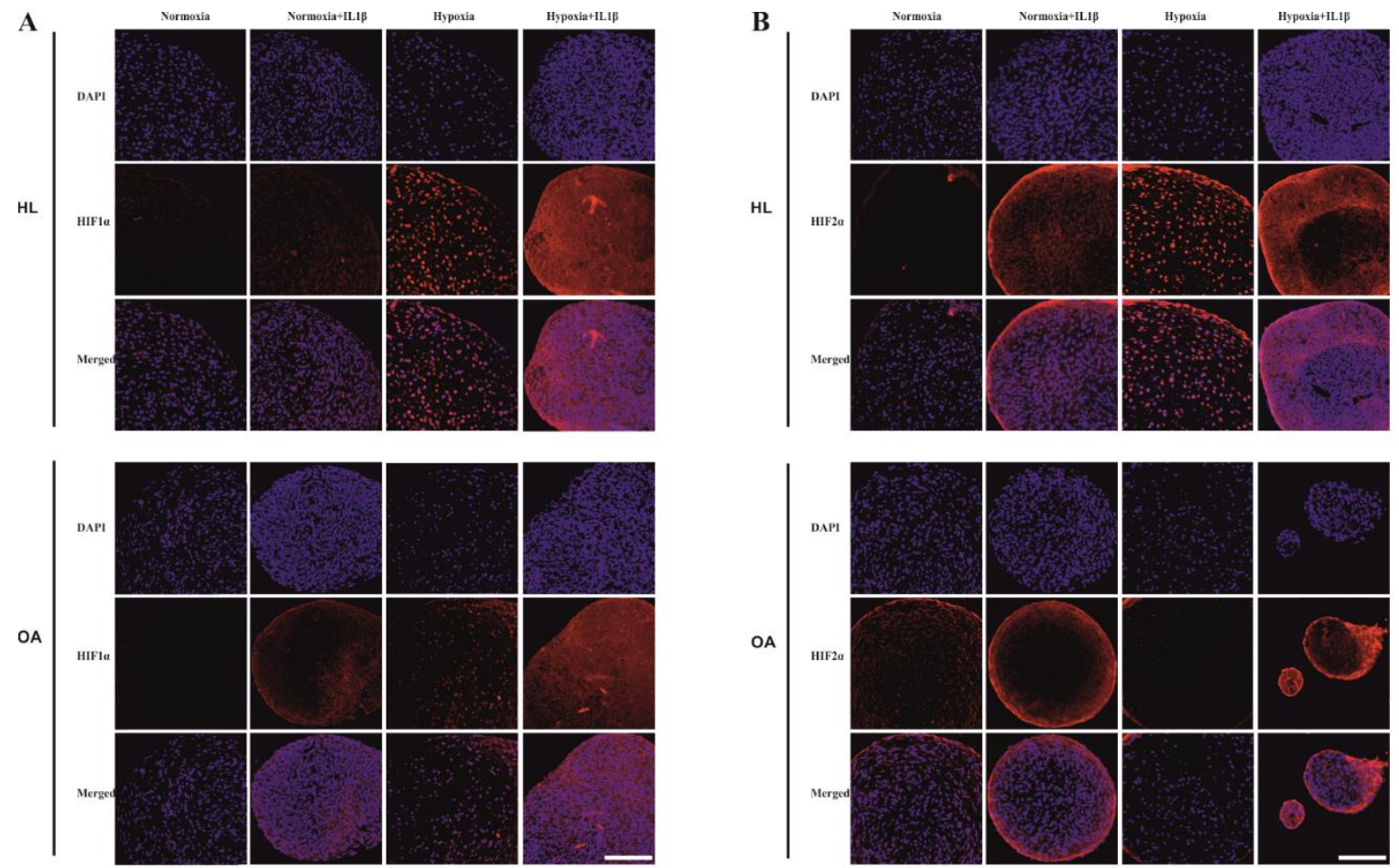

C

HIF-1 $\alpha$

D

HIF-2 $\alpha$
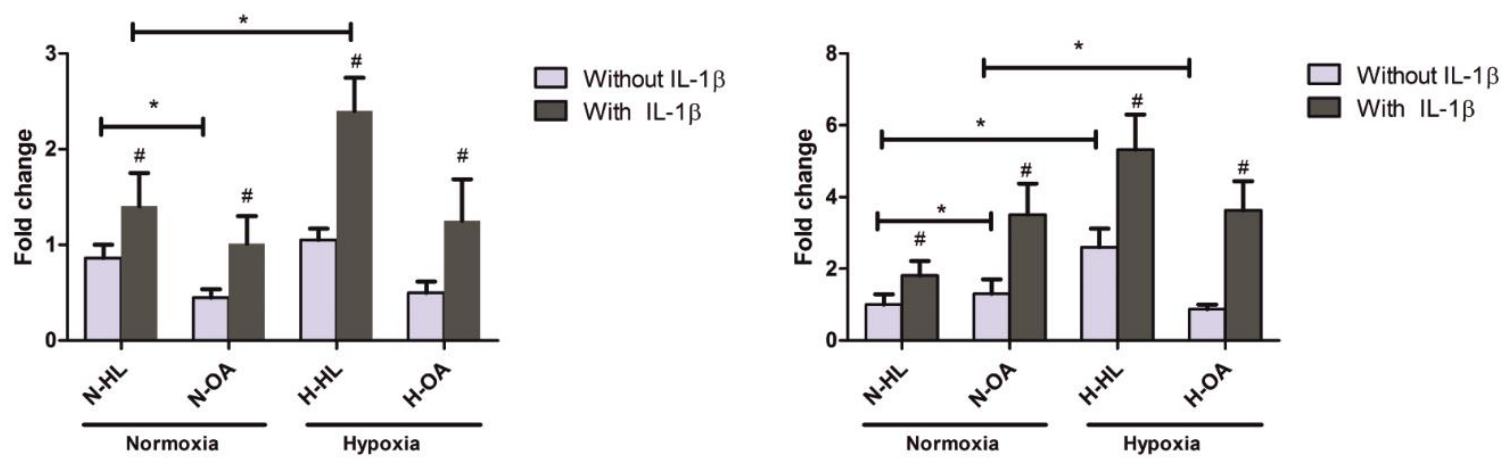

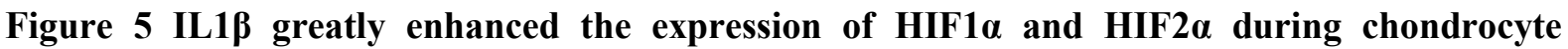
redifferentiation. A. B. Immunofluorescent staining of HIF $1 \alpha$ and HIF $2 \alpha$, respectively. HIF $1 \alpha$ and HIF2 $\alpha$ were detected by rabbit anti-HIF $1 \alpha$ antibody and mouse anti- HIF $2 \alpha$ antibody respectively, followed by anti-mouse or anti-rabbit second antibody coupling by Alex 564 fluorescence. Cell nuclei were counterstained with DAPI. Images were taken by BD pathway confocal microscopy. Scare bar = $250 \mu \mathrm{m}$. HL, healthy chondrocytes; OA, osteoarthritic chondrocytes. C. D. Gene expression of HIF1 $\alpha$ and HIF2 $\alpha$ as assessed by RT-PCR. N-HL, healthy chondrocyte in normoxia; N-OA, OA chondrocyte in normoxia; H-HL, healthy chondrocyte in hypoxia; H-OA, OA chondrocyte in hypoxia. \# represents the significant difference between IL1 $\beta$ stimulation and corresponding control $(p<0.05)$; double \# 
represents $\mathrm{p}<0.01 ; *$ represents $\mathrm{p}<0.05$; double $*$ represents $\mathrm{p}<0.01$. Error bar reflects Standard Deviation (S. D.).

expressed much higher with IL1 $\beta$ stimulation under normoxia. The expression of HIF1 $\alpha$ was greatly enhanced by hypoxia and was present in the nucleus of HL chondrocytes, while it was further enhanced by IL1 $\beta$ supplementation. After spreading out the cells, strongly positive staining was observed in the whole pellet. HIF1 $\alpha$ was barely expressed in the OA chondrocytes under normoxia but greatly induced by IL1 $\beta$ in both normoxia and hypoxia. Likewise, hypoxia promoted HIF1 $\alpha$ expression and translocated into nucleus in OA chondrocytes, but signal intensity was less than in HL chondrocytes. Interestingly, endogenous HIF2 $\alpha$ was hardly observed in the HL chondrocytes but nuclear localized HIF2 $\alpha$ was highly detectable in the hypoxia conditions. OA chondrocytes showed highly endogenous HIF2 $\alpha$ expression in normoxia which was decreased in hypoxia. IL1 $\beta$ profoundly induced HIF2 $\alpha$ expression in both HL and OA chondrocytes under normoxia and hypoxia (Figure 5B).

The gene expression profiles of HIFla and HIF $2 \alpha$ showed a similar trend as the protein staining. The expression of HIF1 $\alpha$ was higher in HL chondrocytes than in OA, and was promoted by IL1 $\beta$ supplementation in both HL and OA chondrocytes under normoxia and hypoxia. Without IL1 $\beta$ stimulation, hypoxia did not significantly induce HIF $\alpha$ expression (Figure 5C). OA chondrocytes displayed higher HIF2 $\alpha$ expression in normoxia but lower expression in hypoxia in comparison with HL chondrocytes. Similarly, IL1 $\beta$ greatly promoted the expression of HIF $2 \alpha$ in both HL and OA chondrocytes under normoxia and hypoxia (Figure $5 \mathrm{D})$.

\section{Discussion}

Accumulating evidence has shown that hypoxia has a positive influence on the chondrocyte phenotype and cartilage matrix formation [16, 17, 35, 36]. However, the difference between healthy and OA chondrocytes during redifferentiation in response to hypoxia is still not clear. In addition, oxygen tension may have an influence not only on chondrocyte redifferentiation, but also on the inflammatory responses of these redifferentiating cells upon stimulation with IL1 $\beta$.

In the present study, we analyzed gene and protein expression profiles of human healthy and OA articular chondrocytes and their matrix production in 3-D cultured pellet in the presence or 
absence of IL1 $\beta$ under normoxia $\left(21 \% \mathrm{O}_{2}\right)$ and hypoxia $\left(2.5 \% \mathrm{O}_{2}\right)$. Before redifferentiation in 3-D pellet in serum free chondrogenic differentiation medium, the chondrocytes were first cultured in monolayer in proliferation medium until passage three. A number of studies have indicated that chondrocytes lose their normal phenotype and potential cartilage formation capability after several passages in vitro, as is reflected by the higher expression of the dedifferentiation marker COL1A1 [3-7]. In order to restore chondrocytes' normal phenotype and function, we cultured the cells in an hypoxia incubator to mimic the chondrocyte environment in vivo. The oxygen concentration in the hypoxia incubator was $2.5 \%$, which is approximate to the physiological oxygen level.

Several studies have reported the positive effects of hypoxia conditions on 3-D chondrocyte redifferentiation [37, 38]. Previously our group have reported that hypoxia inhibits hypertrophic differentiation and endochondral ossification in explanted tibiae [39]. Human mesenchymal stromal cells (MSCs) chondrogenically differentiated in vitro under hypoxia (2.5\% O2) produced more hyaline cartilage [40]. In line with these studies, we observed that hypoxia facilitated chondrocyte redifferentiation and it had a inhibiting effect on the expression of the dedifferentiation marker COL1A1 in both HL and OA chondrocytes. The catabolic genes and hypertrophic markers were greatly inhibited under hypoxic conditions. In addition, we observed that OA chondrocytes benefited more from hypoxia than healthy chondrocytes, which was evidenced by a higher increasing fold of GAG production and Collage type II deposition, as well as the expression of cartilage markers and stronger inhibition of dedifferentiation markers, catabolic markers and hypertrophic markers. Most importantly, hypoxia minimized even abrogated differences between redifferentiated HL and OA chondrocytes present in normoxia.

To assess the different responses of HL and OA chondrocytes to inflammatory factor IL1 $\beta, 10$ $\mathrm{ng} / \mathrm{mL}$ recombinant protein IL1 $\beta$ was added in medium in both normoxia and hypoxia condition. IL1 $\beta$ dramatically inhibited chondrocyte redifferentiation, while it promoted catabolic activity in both normoxia and hypoxia. Under normoxia, with or without IL1 $\beta$, the expression of catabolic genes such as MMPs, ADAMTS4/5 as well as hypertrophic markers was increased in OA cells. This high level of catabolic markers in OA cells was also observed in another study [38]. Interestingly, under normoxia, the expression of ADAMTS 4, MMP13 and COL1OA 1 was downregulated in IL1 $\beta$ treated OA chondrocytes compared to non-treated OA group. This downregulation effect might be mediated by haem oxygenase-1 (HO-1). There 
are studies that have shown that HO-1 could modulate the cytoprotective effects in OA chondrocytes through down-regulation of catabolic factors [41, 42].

Although the absolute expression of MMP1/3/9 under IL1 $\beta$ stimulation was lower in hypoxia, the fold change between no IL1 $\beta$ and IL1 $\beta$ supplementation was higher in hypoxia as compared to normoxia. This indicates that culturing chondrocytes in supraphysiological oxygen tension may induce a stress response that results in mild activation of inflammatory signaling pathways stimulating the relatively high basal expression of catabolic enzymes. This low level of activation of these signaling pathways likely explains the relatively lower level of fold change in chondrocytes in induction of typical IL1 $\beta$ response genes in normoxia. Hypoxia, a more physiological culture condition for chondrocytes, reduces this stress response and inhibits activation of pro-inflammatory pathways resulting in lower levels of catabolic enzymes. It also sentisizes the cells for a challenge with IL1 $\beta$ resulting in higher fold changes. It should be noted that the absolute levels in expression of these catabolic enzymes is still lower in hypoxia as compared to normoxia.

NO is a pro-inflammatory mediator and catabolic factor that contributes to osteoarthritis. It has been reported that NO is induced by pro-inflammatory cytokines and mechanical stress as well as oxygen level [43]. In our study, we found that NO was dramatically induced by IL1 $\beta$. In addition, the expression level of NO production and $i N O S$ was induced by hypoxia in both HL and OA chondrocytes. This result is consistent with previous studies, demonstrating that hypoxia-induced NO protects chondrocytes from damage by hydrogen peroxide [44].

HIF $1 \alpha$ and HIF $2 \alpha$ are the most important hypoxia-induced factors involved in chondrocyte biology $[21,22]$. In this study, we found a higher HIF1 $\alpha$ expression and a lower HIF2 $\alpha$ expression in HL chondrocytes compared to OA chondrocytes under normoxia, which is in line with reports stating that HIF1 $\alpha$ is an anabolic factor and HIF2 $\alpha$ is a catabolic factor [21-23]. Under hypoxia, both HIF1 $\alpha$ and HIF2 $\alpha$ was greatly elevated and translocated into the nucleus in HL chondrocytes. However, hypoxia only enhanced HIF $1 \alpha$ expression but decreased the expression of HIF2 $\alpha$ in OA chondrocytes. Considering the catabolic activity of HIF $2 \alpha$ in OA cartilage, this phenomenon suggests that OA chondrocytes benefited more from hypoxia because the basal HIF $2 \alpha$ expression present in normoxia was inhibited in hypoxia. IL1 $\beta$ greatly induced the cytoplasmic expression of HIF $1 \alpha$ and HIF $2 \alpha$ in both HL and OA chondrocytes, regardless of the oxygen level. IL1 $\beta$ may upregulate HIF $1 \alpha$ via an NF- $\kappa \mathrm{B} / \mathrm{COX}-2$ pathway [45], while IL1 $\beta$ induced HIF $1 \alpha$ is attenuated by p38 MAPK and JNK inhibitors [46]. However, the 
mechanism of IL1 $\beta$ induced HIF2 $\alpha$ expression has not been reported and requires further investigation. This also indicates that both HIF $1 \alpha$ and HIF $2 \alpha$ act as important survival factors when cells live in an inflammatory and hypoxic environment.

In summary, we investigated the functional changes of HL and OA chondrocytes at a cellular level in response to the inflammatory cytokine IL1 $\beta$ under different oxygen tension levels. Hypoxia minimizes the differences between HL and OA chondrocytes, and even restores OA chondrocyte redifferentiation. We provide evidence that normoxia, which is associated with degenerative joint diseases, changes the normal function of chondrocytes by inducing a mild stress response. This mild stress response can be reversed by hypoxia, which reduces the IL1 $\beta$ induced catabolic effects in both cell populations while also sensitizing cell response to IL1 $\beta$ treatment. Our data indicates that the oxygen level may influence inflammation-associated cartilage injury and diseases. Furthermore, our data indicated that chondrocytes derived from healthy areas and clearly OA areas in the joint are differentially susceptible to both oxygen tension and stimulation with the pro-inflammatory cytokine IL1 $\beta$. 


\section{References}

1. Grande, D.A., et al., The repair of experimentally produced defects in rabbit articular cartilage by autologous chondrocyte transplantation. J Orthop Res, 1989. 7(2): p. 20818.

2. Vasara, A.I., et al., Immature porcine knee cartilage lesions show good healing with or without autologous chondrocyte transplantation. Osteoarthritis Cartilage, 2006. 14(10): p. 1066-74.

3. Egli, R.J., et al., Hypoxic expansion promotes the chondrogenic potential of articular chondrocytes. J Orthop Res, 2008. 26(7): p. 977-85.

4. $\quad$ von der Mark, K., et al., Relationship between cell shape and type of collagen synthesised as chondrocytes lose their cartilage phenotype in culture. Nature, 1977. 267(5611): p. 531-2.

5. Benya, P.D. and J.D. Shaffer, Dedifferentiated chondrocytes reexpress the differentiated collagen phenotype when cultured in agarose gels. Cell, 1982. 30(1): p. 215-24.

6. Watt, F.M., Effect of seeding density on stability of the differentiated phenotype of pig articular chondrocytes in culture. J Cell Sci, 1988. 89 ( Pt 3): p. 373-8.

7. Hong, E. and A.H. Reddi, Dedifferentiation and redifferentiation of articular chondrocytes from surface and middle zones: changes in microRNAs-221/-222, -140, and -143/145 expression. Tissue Eng Part A, 2013. 19(7-8): p. 1015-22.

8. Holtzer, H., et al., THE LOSS OF PHENOTYPIC TRAITS BY DIFFERENTIATED CELLS IN VITRO, I. DEDIFFERENTIATION OF CARTILAGE CELLS. Proc Natl Acad Sci U S A, 1960. 46(12): p. 1533-42.

9. Hsieh-Bonassera, N.D., et al., Expansion and redifferentiation of chondrocytes from osteoarthritic cartilage: cells for human cartilage tissue engineering. Tissue Eng Part A, 2009. 15(11): p. 3513-23.

10. Tallheden, T., et al., Gene expression during redifferentiation of human articular chondrocytes. Osteoarthritis Cartilage, 2004. 12(7): p. 525-35.

11. Dehne, T., et al., Chondrogenic differentiation potential of osteoarthritic chondrocytes and their possible use in matrix-associated autologous chondrocyte transplantation. Arthritis Res Ther, 2009. 11(5): p. R133.

12. Tallheden, T., et al., Proliferation and differentiation potential of chondrocytes from osteoarthritic patients. Arthritis Res Ther, 2005. 7(3): p. R560-8. 
13. Silver, I.A., Measurement of $\mathrm{pH}$ and ionic composition of pericellular sites. Philos Trans R Soc Lond B Biol Sci, 1975. 271(912): p. 261-72.

14. Ogata, K., L.A. Whiteside, and P.A. Lesker, Subchondral route for nutrition to articular cartilage in the rabbit. Measurement of diffusion with hydrogen gas in vivo. $\mathrm{J}$ Bone Joint Surg Am, 1978. 60(7): p. 905-10.

15. Rajpurohit, R., et al., Adaptation of chondrocytes to low oxygen tension: relationship between hypoxia and cellular metabolism. J Cell Physiol, 1996. 168(2): p. 424-32.

16. Sheehy, E.J., C.T. Buckley, and D.J. Kelly, Oxygen tension regulates the osteogenic, chondrogenic and endochondral phenotype of bone marrow derived mesenchymal stem cells. Biochem Biophys Res Commun, 2012. 417(1): p. 305-10.

17. Hirao, M., et al., Oxygen tension regulates chondrocyte differentiation and function during endochondral ossification. J Biol Chem, 2006. 281(41): p. 31079-92.

18. Mariani, E., L. Pulsatelli, and A. Facchini, Signaling pathways in cartilage repair. Int J Mol Sci, 2014. 15(5): p. 8667-98.

19. Kanichai, M., et al., Hypoxia promotes chondrogenesis in rat mesenchymal stem cells: a role for AKT and hypoxia-inducible factor (HIF)-1alpha. J Cell Physiol, 2008. 216(3): p. 708-15.

20. Robins, J.C., et al., Hypoxia induces chondrocyte-specific gene expression in mesenchymal cells in association with transcriptional activation of Sox9. Bone, 2005. 37(3): p. 313-22.

21. Strobel, S., et al., Anabolic and catabolic responses of human articular chondrocytes to varying oxygen percentages. Arthritis Res Ther, 2010. 12(2): p. R34.

22. Saito, T., et al., Transcriptional regulation of endochondral ossification by HIF-2alpha during skeletal growth and osteoarthritis development. Nat Med, 2010. 16(6): p. 67886.

23. Yang, S., et al., Hypoxia-inducible factor-2alpha is a catabolic regulator of osteoarthritic cartilage destruction. Nat Med, 2010. 16(6): p. 687-93.

24. Ryu, J.H., et al., Hypoxia-inducible factor-2alpha regulates Fas-mediated chondrocyte apoptosis during osteoarthritic cartilage destruction. Cell Death Differ, 2012. 19(3): p. 440-50.

25. Goldring, M.B., The role of the chondrocyte in osteoarthritis. Arthritis Rheum, 2000. 43(9): p. 1916-26.

26. Heinegard, D. and T. Saxne, The role of the cartilage matrix in osteoarthritis. Nat Rev Rheumatol, 2011. 7(1): p. 50-6. 
27. Scanzello, C.R. and S.R. Goldring, The role of synovitis in osteoarthritis pathogenesis. Bone, 2012. 51(2): p. 249-57.

28. Kobayashi, M., et al., Role of interleukin-1 and tumor necrosis factor alpha in matrix degradation of human osteoarthritic cartilage. Arthritis Rheum, 2005. 52(1): p. 12835.

29. Wojdasiewicz, P., L.A. Poniatowski, and D. Szukiewicz, The role of inflammatory and anti-inflammatory cytokines in the pathogenesis of osteoarthritis. Mediators Inflamm, 2014. 2014: p. 561459.

30. Tetlow, L.C., D.J. Adlam, and D.E. Woolley, Matrix metalloproteinase and proinflammatory cytokine production by chondrocytes of human osteoarthritic cartilage: associations with degenerative changes. Arthritis Rheum, 2001. 44(3): p. 585-94.

31. Kapoor, M., et al., Role of proinflammatory cytokines in the pathophysiology of osteoarthritis. Nat Rev Rheumatol, 2011. 7(1): p. 33-42.

32. Wu, L., et al., Serum regulates adipogenesis of mesenchymal stem cells via MEK/ERKdependent PPARgamma expression and phosphorylation. J Cell Mol Med, 2010. 14(4): p. 922-32.

33. $\mathrm{Wu}, \mathrm{L}$., et al., Trophic effects of mesenchymal stem cells increase chondrocyte proliferation and matrix formation. Tissue Eng Part A, 2011. 17(9-10): p. 1425-36.

34. Du, Q., et al., Regulation of human nitric oxide synthase 2 expression by Wnt betacatenin signaling. Cancer Res, 2006. 66(14): p. 7024-31.

35. Lafont, J.E., S. Talma, and C.L. Murphy, Hypoxia-inducible factor 2alpha is essential for hypoxic induction of the human articular chondrocyte phenotype. Arthritis Rheum, 2007. 56(10): p. 3297-306.

36. Murphy, C.L. and J.M. Polak, Control of human articular chondrocyte differentiation by reduced oxygen tension. J Cell Physiol, 2004. 199(3): p. 451-9.

37. Babur, B.K., et al., The interplay between chondrocyte redifferentiation pellet size and oxygen concentration. PLoS One, 2013. 8(3): p. e58865.

38. Markway, B.D., H. Cho, and B. Johnstone, Hypoxia promotes redifferentiation and suppresses markers of hypertrophy and degeneration in both healthy and osteoarthritic chondrocytes. Arthritis Res Ther, 2013. 15(4): p. R92.

39. Leijten, J.C., et al., Hypoxia inhibits hypertrophic differentiation and endochondral ossification in explanted tibiae. PLoS One, 2012. 7(11): p. e49896. 
40. Leijten, J., et al., Metabolic programming of mesenchymal stromal cells by oxygen tension directs chondrogenic cell fate. Proc Natl Acad Sci U S A, 2014. 111(38): p. 13954-9.

41. Guillen, M., et al., Haem oxygenase-1 regulates catabolic and anabolic processes in osteoarthritic chondrocytes. J Pathol, 2008. 214(4): p. 515-22.

42. Megias, J., et al., Heme oxygenase-1 induction modulates microsomal prostaglandin E synthase-1 expression and prostaglandin E(2) production in osteoarthritic chondrocytes. Biochem Pharmacol, 2009. 77(12): p. 1806-13.

43. Fermor, B., et al., Oxygen, nitric oxide and articular cartilage. Eur Cell Mater, 2007. 13: p. 56-65; discussion 65.

44. Matsushita, T., et al., Hypoxia-induced nitric oxide protects chondrocytes from damage by hydrogen peroxide. Inflamm Res, 2004. 53(8): p. 344-50.

45. Jung, Y.J., et al., IL-Ibeta-mediated up-regulation of HIF-lalpha via an NFkappaB/COX-2 pathway identifies HIF-1 as a critical link between inflammation and oncogenesis. FASEB J, 2003. 17(14): p. 2115-7.

46. Murata, M., et al., Distinct signaling pathways are involved in hypoxia- and IL-1induced VEGF expression in human articular chondrocytes. J Orthop Res, 2006. 24(7): p. $1544-54$. 


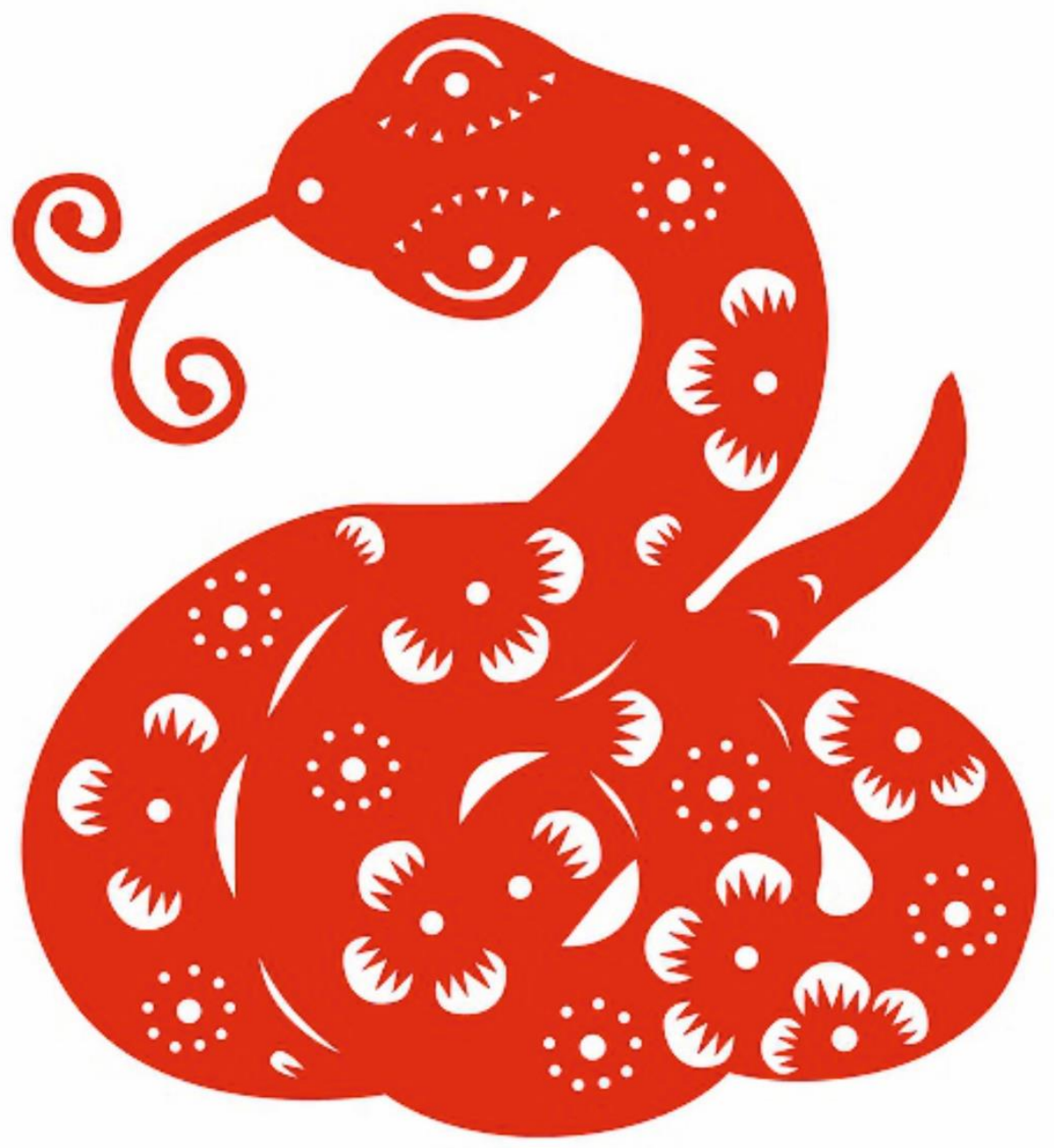




\section{Chapter 6}

\section{Co-treatment of TGF- $\beta 3$ and BMP-7 is superior in stimulating chondrocyte redifferentiation in both hypoxia and normoxia compared to single treatments}

Xiaobin Huang, Leilei Zhong, Janine N. Post and Marcel Karperien

Developmental BioEngineering, MIRA Institute for Biomedical Technology and Technical Medicine, University of Twente, Enschede 7500 AE, The Netherlands

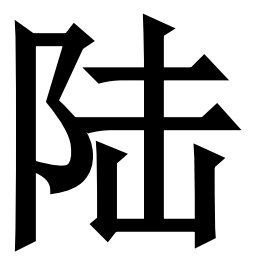

三十辐共一毂, 当其无, 有车之用。

We put thirty spokes together and call it a wheel. But it is on the space where there is nothing. That the usefulness of the wheel depends. 


\begin{abstract}
Signaling by members of the transforming growth factor- $\beta$ (TGF- $\beta$ ) superfamily, such as TGF$\beta 3$ and BMP7, and by oxygen tension play each a pivotal role in chondrocyte biology. However, their cross talk has hardly been studied in particular during redifferentiation of culture expanded dedifferentiated osteoarthritic chondrocytes. The objective of this research was to elucidate the endogenous BMP7 expression in human OA cartilage and the effect of oxygen tension on the single or combined treatment with TGF- $\beta 3$ and BMP7 on OA chondrocyte redifferentiation in three dimensional (3D) pellet cultures. The results showed the expression of BMP7 and its intracellular signaling target SMAD1/5/8 decreases in early OA while it increases in late stage of OA. TGF- $\beta 3$ or BMP7 alone were not as efficient in redifferentiating chondrocytes as the combined treatment with TGF- $\beta 3$ and BMP-7 both in normoxia and hypoxia, reflected by Alcian blue/Safranin O staining and collagen type II (COL2A1) protein expression, as well as gene expression of COL2A1, Aggrecan (ACAN). Hypoxia elevated TGF$\beta 3$ and BMP7-induced matrix formation of OA chondrocytes and alleviated the catabolic gene expression of matrix metalloproteinase $(M M P)-1,3,13$. Interestingly, cells cultured under normoxia displayed mild signs of an inflammatory stress response based on gene expression analysis which was effectively counteracted by culturing the cells under low oxygen tension. Our data underscores the important modulatory role of oxygen tension on the chondrocyte's responsiveness to TGF- $\beta 3$ and/or BMP7.
\end{abstract}

Key words: TGF- $\beta 3$, BMP7, hypoxia, osteoarthritis, redifferentiation, ACI, cartilage, tissue engineering 


\section{Introduction}

Articular cartilage exhibits a poor self-regeneration capacity, even minor cartilage defects may predispose to early onset osteoarthritis. To intervene with this vicious cycle, surgical intervention is often considered. Several surgical strategies have been developed to repair damaged cartilage. One of the most promising approaches is autologous chondrocyte implantation (ACI) [1]. This approach is based on the isolation of chondrocytes from the nonweight bearing healthy area of articular cartilage, followed by culture expansion in monolayer for several weeks to obtain sufficient cells for implantation into the lesion. However, monolayer expansion leads to a rapid loss of typical chondrocyte features, a process known as chondrocyte dedifferentiation which eventually results in fibrous cartilage formation rather than hyaline cartilage after implantation, [2-5]. Consequently, it is important to optimize culture conditions to minimize the negative aspects of chondrocyte dedifferentiation or, alternatively develop effective strategies for chondrocyte redifferentiation.

The Transforming Growth Factor- $\beta$ (TGF- $\beta$ ) superfamily is a family of secreted signaling factors including TGF- $\beta$ and bone morphogenetic proteins (BMPs) with a remarkable ability to induce cartilage and bone formation. TGF- $\beta$ s and BMPs bind to specific receptors at the cell surface to initiate intracellular signal molecules of the Sma and Mad related proteins (SMADs). Generally TGF- $\beta$ induced signaling depends on Smad2 and Smad3 $[6,7]$ resulting in stabilization of the SOX9 transcription complex meanwhile inhibiting RUNX2 expression [8, 9]. BMP signaling depends on the Smad1/5/8 pathway to stimulate the expression of typical hypertrophic markers, like COL1OA1, MMP13 and ALPL during chondrogenesis of mesenchymal stem cells (MSCs) and termimal differentiation of primary chondrocytes [10]. TGF- $\beta$ is an effective inducer of chondrogenesis $[11,12]$ by stimulating chondrocyte proliferation while inhibiting chondrocyte hypertrophy and maturation [13-18] in vitro. However, there is also conflicting evidence claiming that TGF $\beta 1$ administration can redirect expanded human articular chondrocytes towards a more hypertrophic phenotype [19]. Moreover, TGF $\beta$ was reported to induce synovial lining cells to produce inflammatory factors, such as IL1 $\beta$ and TNF $\alpha$, which further stimulate articular chondrocyte dedifferentiation and stimulating hypertrophy by facilitating the depositing of collagen type $\mathrm{X}$ instead of collagen type II and aggrecan [20]. Indeed the complex and sometimes opposing role of TGF- $\beta$ superfamily members and their downstream effectors in chondrocyte hypertrophy has recently 
been reviewed [21]. It can however be concluded that TGF- $\beta$ alone is insufficient to preserve the chondrocyte phenotype in cartilage tissue engineering.

Like TGF $\beta$ s, BMPs are multi-functional cytokines that belong to the TGF- $\beta$ superfamily. They are indispensable for early cartilage formation in the embryo [22]. They are also potent regulators of chondrocyte hypertrophy and matrix degradation [23-26]. This is particularly true for commonly used BMP2 and BMP4. The mode of action of BMP7 is however distinct. This factor stimulates chondrocyte proliferation and inhibits chondrocyte hypertrophy [21, 27, 28]. The recombinant human version of BMP7 is clinically approved and used to promote osteogenesis at sites of poor fracture healing and to enhance integration of bone grafts [29]. Furthermore, intra-articular injection of BMP7 has been considered as a regenerative therapy for osteoarthritis. There are only a few reports that have studied the effect of combination treatment of TGF- $\beta$ and BMPs in chondrocyte (de)differentiation. Shintani and coworkers reported that TGF- $\beta 1$ enhanced BMP-2 induced chondrogenesis of bovine synovial explants resulting in improved hyaline-cartilage like properties of the neocartilage [30]. Toh et al. demonstrated that the combination of BMP2 and TGF- $\beta 1$ resulted in a marked enhancement of collagen II synthesis and matrix proteoglycan deposition in chondrogenically differentiation of MSCs isolated from New Zealand white rabbits [31]. BMP2 also enhanced the TGF- $\beta 3$ induced chondrogenesis of human bone marrow MSCs [32]. Nakayama et al. reported that TGF- $\beta 3$ synergistically enhanced BMP4 induced cartilage formation in embryonic stem cell derived mesodermal cells and similar results have been reported for BMP6 [33]. Miyamoto et al elucidated that BMP7 enhanced chondrogenesis of synovial MSCs when combined with TGF$\beta 1$ [34]. There are also studies reporting that chondrogenic differentiation medium supplemented with BMP-7 only in the absence of TGF- $\beta 1$ hardly enhanced chondrogenic differentiation. This is in marked contrast to the condition in which both growth factors were added simultaneously to chondrogenically differentiating MSCs [35]. Remarkably, in contrast to BMP2, 4 and 6 which promote chondrocyte hypertrophy, BMP7 may inhibit this process. The exact mechanism is still unknown $[27,28]$.

However, all these studies have been performed in normoxia $\left(21 \% \mathrm{O}_{2}\right)$ which is a supraphysiological oxygen concentration compared to the much lower oxygen tension in articular cartilage in vivo. Articular cartilage is a typical avascular tissue, and chondrocytes are used to live in low oxygen environments [36]. Hypoxia has a positive influence on the chondrocyte phenotype and has been shown to stimulate cartilage matrix formation [37, 38]. Furthermore, 
most studies have focused on chondrogenic differentiation of MSCs from different sources with treatment of BMPs or TGF- $\beta$ s. Only a limited number of studies has focused on the redifferentiation of chondrocytes after culture expansion in monolayer under hypoxic conditions in the presence of these factors. The aim of this study was to identify the effects of exogenous BMP7 and TGF- $\beta 3$ on the redifferentiation of osteoarthritic chondrocytes after expansion in monolayer, using an aggregate pellet culture in low oxygen condition, and to explore the possibility to use the combination of hypoxia with TGF $\beta$ and BMP7 to stimulate chondrocyte redifferentiation.

\section{Materials and Methods}

\section{Cartilage samples collection}

The collection and use of human cartilage was approved by the local hospital ethical committee and for all samples informed written consent was obtained. Cartilage specimens were isolated from 12 patients (mean age \pm SD: $68 \pm 6$ years) with osteoarthritis (OA) undergoing total knee replacement surgery. In order to get comparable cartilage samples, several cartilage pieces were removed from the same joint and for each of the specimens a histological cartilage score was determined as previously described [39].

\section{Cell culture and expansion}

Human primary chondrocytes (hChs) were obtained from macroscopically healthy looking full thickness cartilage, dissected from knee biopsies of four OA patients (mean \pm SD age $60 \pm 3$ years) undergoing total knee replacement, as described previously [40]. To isolate cells, the cartilage was digested in chondrocyte proliferation medium containing $0.15 \%$ collagenase type II (Worthington) for 20-22 hours (hrs). Subsequently the hChs were expanded at a density of 3000 cells $/ \mathrm{cm}^{2}$ in chondrocyte proliferation medium until the monolayer reached $80 \%$ confluency. Chondrocyte proliferation medium consisted of DMEM supplemented with 10\% fetal bovine serum (FBS), 1×non-essential amino acids, $0.2 \mathrm{mM}$ ascorbic acid 2-phosphate (AsAP), $0.4 \mathrm{mM}$ proline, $100 \mathrm{U} / \mathrm{mL}$ penicillin and $100 \mu \mathrm{g} / \mathrm{ml}$ streptomycin. The hChs were used in passage 3 unless otherwise stated. 


\section{Pellet cultures and chondrogenic differentiation}

Pellets consisting of $2.5 \times 10^{5}$ cells were cultured for 3 weeks at $37{ }^{\circ} \mathrm{C}, 5 \% \mathrm{CO}_{2}, 21 \% \mathrm{O}_{2}$ (for nomoxia) or $2.5 \% \mathrm{O}_{2}$ (for hypoxia), in chondrogenic differentiation medium (DMEM supplemented with $50 \mu \mathrm{g} / \mathrm{mL}$ ITS-premix, $50 \mu \mathrm{g} / \mathrm{mL}$ of AsAP, $100 \mu \mathrm{g} / \mathrm{mL}$ of sodium pyruvate, $10^{-7} \mathrm{M}$ of dexamethasone, $100 \mathrm{U} / \mathrm{mL}$ of penicillin and $100 \mu \mathrm{g} / \mathrm{ml}$ of streptomycin). The medium was supplemented with $10 \mathrm{ng} / \mathrm{mL}$ TGF- $\beta 3$ or $100 \mathrm{ng} / \mathrm{ml}$ BMP7 (R\&D). The medium, with cytokines supplements, was refreshed twice per week.

\section{Total RNA extraction and quantitative polymerase chain reaction (qPCR)}

Cell pellets were crashed and RNA was isolated using the Trizol reagent (Thermo Fisher Scientific). The concentration and purity of RNA samples were determined using the Nanodrop 2000 (Thermo scientific). Total mRNA was reverse-transcribed into cDNA using the iScript cDNA Synthesis kit (Bio-Rad). qPCR was performed using the SYBR Green sensimix (Bioline). PCR Reactions were carried out using the Bio-Rad CFX96 (Bio-Rad) under the following conditions: cDNA was denatured for $5 \mathrm{~min}$ at $95^{\circ} \mathrm{C}$, followed by 39 cycles consisting of $15 \mathrm{~s}$ at $95^{\circ} \mathrm{C}, 15 \mathrm{~s}$ at $60^{\circ} \mathrm{C}$ and $30 \mathrm{~s}$ at $72^{\circ} \mathrm{C}$. For each reaction a melting curve was generated to test primer dimer formation and non-specific priming.

\section{Alcian blue and Safranin O staining}

Cartilage samples were fixed in $10 \%$ phosphate buffered formalin $(\mathrm{pH}=7)$ overnight at room temperature (RT), dehydrated with a graded ethanol series and embedded in paraffin using routine procedures. Sections were cut $5 \mu \mathrm{m}$ thick using a microtome (Shandon). Before staining, the slides were de-paraffinized in xylene and rehydrated with graded ethanols. Cell pellets samples were collected after 5 weeks of incubation and fixed with $10 \%$ buffered formalin for 2 hours at RT. Sections of $7 \mu \mathrm{m}$ thick were directly cut using a cryotome (Shandon) after embedding in cryomatrix. Slides were either stained for sulfated glycosaminoglycans (GAG) with a $0.5 \%$ w/v solution of Alcian blue ( $\mathrm{pH}=1$, adjusted with $\mathrm{HCl}$ ) for $30 \mathrm{~min}$, or stained for sulfated GAG with a $0.1 \%$ solution of Safranin O for 5 min (Sigma Aldrich). The samples were then counterstained with nuclear fast red $(0.1 \% \mathrm{w} / \mathrm{v}$ of nuclear fast red in $5 \%$ aluminum sulfate) for 5 min [41], or counterstained with haematoxylin (Sigma Aldrich) dissolved in water. 


\section{Immunofluorescent (IF) staining for collagen type II}

Immunofluorescent staining of collagen type II was performed using $7 \mu \mathrm{m}$ sections from pellets.. After rehydration into deionized $\mathrm{H}_{2} \mathrm{O} 30 \mathrm{~min}$, samples were pre-incubated with $5 \mu \mathrm{g} / \mathrm{mL}$ proteinase K (Sigma Aldrich) in TE buffer for $10 \mathrm{~min}$ at RT followed by treatment with 1 $\mathrm{mg} / \mathrm{mL}$ hyaluronidase (Sigma Aldrich) in $1 \%$ BSA in PBS for 40 min at $37^{\circ} \mathrm{C}$. Samples were blocked in 5\% BSA in PBS for 1 hour, then incubated with rabbit anti human collagen II antibody (ab34712, Abcam), which was diluted 1:100 in 5\% BSA in PBS overnight at $4^{\circ}$. Sections were rinsed with PBS Tween 20 (1\%) for 3 times, 5min/time. Then Alexa®Fluor 546labelled goat anti-rabbit antibody (Invitrogen) 1:100 in 5\% BSA in PBS was added and incubated for 2 hours at RT. Samples were rinsed with PBS and embedded using mounting medium with DAPI. Slides were viewed by BD pathway confocal microscopy. .

\section{Immunohistochemistry (IHC) of BMP7 and SMAD 1/5/8 in cartilage or pellets}

Immunohistochemistry staining of BMP7 and SMAD 1/5/8 for cartilage samples was performed using $5 \mu \mathrm{m}$ sections, and the staining of SMAD 1/5/8 for pellet samples using $7 \mu \mathrm{m}$ sections. The sections were pre-incubated with $5 \mu \mathrm{g} / \mathrm{mL}$ proteinase K (Sigma Aldrich) for 10 min followed by $1 \mathrm{mg} / \mathrm{mL}$ hyaluronidase (Sigma Aldrich) for $40 \mathrm{~min}$ at $37{ }^{\circ} \mathrm{C}$ dissolved in PBS. Mouse anti-human BMP7 (PeproTech) or mouse anti-human SMAD 1/5/8 antibody (Sata Ccurz biotechnology) was diluted 1:100 in PBS with 5\% BSA and were incubated overnight at $4{ }^{\circ} \mathrm{C}$. Non-immune controls underwent the same procedure without primary antibody incubation. The biotinylated secondary antibody was diluted 1:500 in 5\% BSA in PBS and incubated for 30 minutes at RT. HRP-Streptavidin was added and incubated for 30 minutes at RT. For visualization, DAB substrate kit was used (ab64238, Abcam).Cell nuclei were counterstained with hematoxylin. Images were taken by using a Nanozoomer (Iwata City, Japan).

\section{EdU and TUNEL staining}

For labeling of newly synthesized DNA in proliferating cells, EdU (5-ethynyl-2'-deoxyuridine) was added to the culture media at a concentration of $10 \mu \mathrm{M}, 24$ hours before harvesting the samples. Cell pellets were then washed with PBS and fixed with $10 \%$ buffered formalin for 15 min. Samples were embedded in cryomatrix, and cut into $7 \mu \mathrm{M}$ sections with a cryotome 
(Shandon). Sections were permeabilized and stained for EdU with Click-iT® EdU Imaging Kit (ThermoFisher scientific). Cryo-sections were also stained for DNA fragments with DeadEnd Fluorometric TUNEL System (Promega). Nuclei were counterstained with Hoechst 33342.

\section{GAG and DNA assays}

After redifferentiation, pellets were digested in $250 \mu \mathrm{l}$ of Tris- $\mathrm{HCl}$ buffer $(0.05 \mathrm{M}$ Tris, $1 \mathrm{mM}$ $\mathrm{CaCl} 2$, pH 8.0) supplemented with $1 \mathrm{mg} / \mathrm{ml}$ proteinase $\mathrm{K}$ (Roche) for $16 \mathrm{hrs}$ at $56{ }^{\circ} \mathrm{C}$. Diluted samples $(25 \mu \mathrm{l})$ were mixed with $150 \mu \mathrm{l}$ 1,9-dimethylmethylene blue (DMMB)-dye solution (3.04 g/l glycine, $2.38 \mathrm{~g} / 1 \mathrm{NaCl}, 20 \mathrm{mg} / \mathrm{l} \mathrm{DMMB}$ in distilled water) and absorbance was measured at $525 \mathrm{~nm}$. Relative cell number was determined by quantification of total DNA using a QuantiFluor ${ }^{\circledR}$ dsDNA System kit ( Promega), according to the manufacturer's instructions.

\section{Enzyme- linked immunosorbent assay (ELISA)}

The supernatant of pellet cultures was collected every week. The content of MMP1 dissolved in medium was measured by ELISA using a mouse anti-human MMP1 antibody (MAB901SP, R\&D systems), followed by incubation with a rabbit anti-mouse antibody coupled to a peroxidase. Signals were developed by the addition of Tetramethylbenzidine according to the manufacturer's protocol (TMB, 1-Step Ultra TMB-ELISA, Thermo Scientific). The reactions were stopped by the addition of $1 \mathrm{M} \mathrm{H}_{2} \mathrm{SO}_{4}$ and samples were measured at $450 \mathrm{~nm}$ (Micro Plate Reader).

\section{Statistical analysis}

Four OA chondrocyte donors were used for cell pellet experiments. Each experiment was performed in triplicate. Statistical differences between two groups were analyzed by two-tailed student's t-tests or one-way ANOVA. $\mathrm{P}<0.05$ was considered statistically significant and indicated with an asterisk. Data are expressed as the mean $\pm \mathrm{SD}$. 


\section{Results}

\section{The expression of BMP7 and SMAD1/5/8 decreases in early OA while it increases in late stage of OA}

To better characterize the BMP7/SMAD signaling pathway and its involvement in cartilage degeneration, we performed IHC to detect protein expression of BMP7 and its downstream target SMAD1/5/8 in OA cartilage. The cartilage samples showed visual changes typical for OA with the reduction of Alcian blue and Safranin O staining and the presence of fissures. The OARSI score was used to determine OA severity [39]. The expression of BMP7 (Figure 1A) distinctly decreased with increasing severity from 0 to 3 in the early OA development, while increased from grade 3 to 5. BMP7 was mainly expressed in the matrix of the superficial layer and highly expressed inside chondrocytes. BMP7 expression was decreased in the middle layer and deep layers, however it was increased again in hypertrophic chondrocytes. In the early OA stage from grade 0 to 2, positive staining of BMP7 was observed in the whole cartilage section, high to low from superficial layer to deep layer. At grade 3, intensity of BMP7 staining was decreased. BMP7 was only detected in chondrocytes in the superficial layer and in hypertrophic chondrocytes in the deep layer. Interestingly, in cartilage specimens with OA stage 4 and 5, and especially in grade 5 specimens, BMP7 was highly expressed in cell clusters, which is a typical characteristic of late OA. The expression of BMP7 was measured in each patient (Supplemental Figure 1)

SMAD1/5/8 expression showed a similar trend in expression as BMP7 (Figure 1B). It gradually declined from grade 0 to 3, and increased from grade 3 to 5. SMAD1/5/8 was mainly present in the chondrocytes of the superficial layer, even in the relatively health cartilage of grade 0 and 1. In stage 2 to $4 \mathrm{OA}$ specimens, positive staining of SMAD1/5/8 was hard to observe in the chondrocytes of the middle layer and in hypertrophic chondrocytes of the deep layer unlike BMP7. However, at stage 5, SMAD1/5/8 was strongly expressed in the top layer of the cartilage and especially in the chondrocyte clusters. The expression of SMAD1/5/8 was measured in each patient (Supplemental Figure 2) 
A

G0

G1

G2

G3

G4
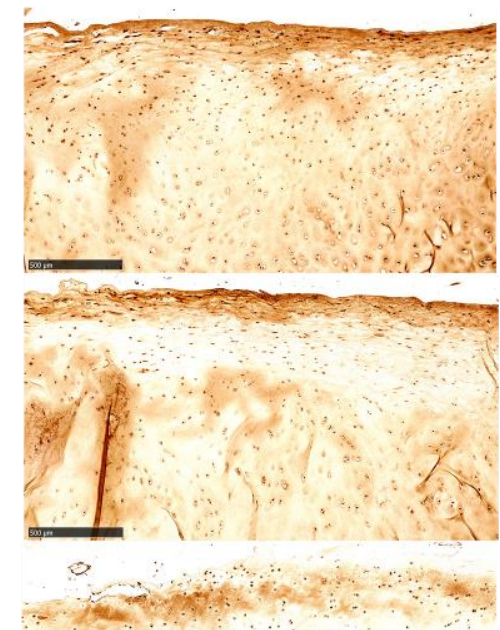

$\because \ldots$

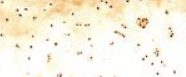

.
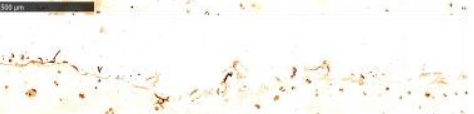

G5
B
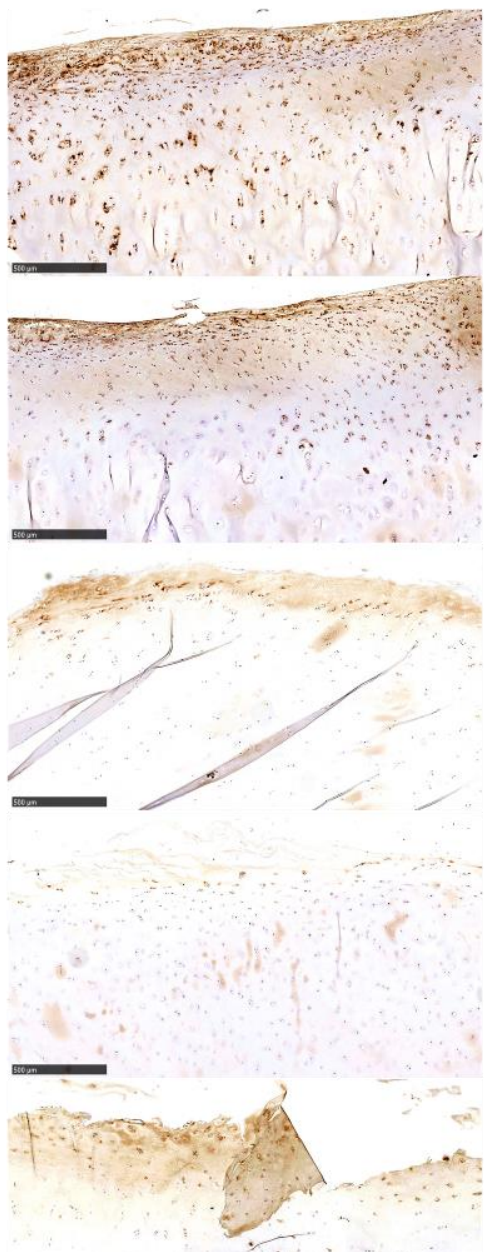

$4 X$

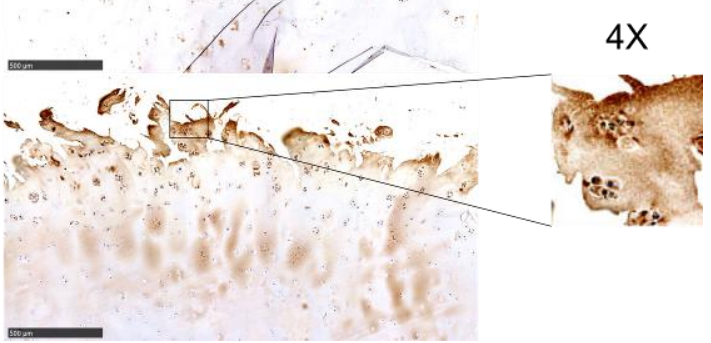

Figure 1 The protein expression of BMP7 and SMAD1 in human cartilage was visualized by IHC in each donor. The expression of BMP7 (A) and SMAD 1/5/8 (B) was visualized in differentially graded cartilage specimens of OA patients. Representative pictures are shown. Images were taken using

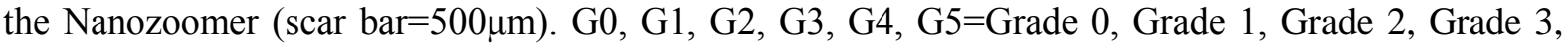
Grade 4, Grade 5. Magnified pictures were indicated in inserts. 


\section{Hypoxia enhanced matrix formation of OA chondrocytes with TGF- $\beta 3$ or BMP7 treatment}

Due to the dedifferentiation of chondrocytes when cultured in monolayer, chondrocyte redifferentiation is required before or after implantation in the patient to repair the cartilage defect. In this research, 3D pellet culture in chondogenic differentiation medium supplemented with TGF- $\beta 3$, or BMP7 or both under normoxia and hypoxia was used to induce redifferentiation. The size of pellets with either TGF- $\beta 3$ or BMP7 treatment under hypoxia was obviously larger than pellets cultured under normoxia (Figure 2A and 2B). There is no significant difference in the TGF- $\beta 3$ and BMP7 treated groups between normoxia and hypoxia.

In order to clarify whether the enhanced pellet size is due to cell proliferation or to matrix production, EdU proliferation assays, Alcian blue and Safranin O staining and GAG assay were performed. As shown in Figure $2 \mathrm{C}$ and $2 \mathrm{D}$, the proliferation ratio after treatment with TGF- $\beta 3$ did change between normoxia and hypoxia, while BMP7 treated pellets under hypoxia presented less positive Edu staining than pellets in normoxia. Moreover, In the treatment of TGF- $\beta 3$ or BMP7, pellets of hypoxia showed lower cell density than pellets in normoxia as can be derived from the DAPI staining. TUNEL assay showed the amount of apoptotic cells was increased in hypoxia for TGF- $\beta 3$ treatment and no difference for BMP7 treatment group compared to normoxia (Figure $2 \mathrm{E}$ and $2 \mathrm{~F}$ ). However, GAG deposition was increased in hypoxia after treatment with TGF- $\beta 3$ or BMP7 (Figure $3 \mathrm{~A}$ and $3 \mathrm{~B}$ ). This was confirmed by GAG assays ( Figure 3C). Although the amount of DNA showed no difference between hypoxia and normoxia, GAG production in hypoxia was much higher than in normoxia up to approximately 3 fold after treatment with TGF- $\beta 3$ or BMP7. These observations indicated that the enlarged pellet size in hypoxia after treatment with TGF- $\beta 3$ or BMP7 was mainly due to increased matrix production rather than increased cell proliferation.

\section{Synergistic effect of TGF-ß3 and BMP7 on chondrocytes redifferentiation}

As Figure 2A and 2B shows, treatment with TGF- $\beta 3$ or BMP7 alone did not significantly increase the pellet size in normoxia in contrast to pellets treated in hypoxia. Interestingly, the combined treatment significantly increased pellet size compared to the single treatments. The effect was more pronounced in normoxia than in hypoxia. EdU staining indicated that the treatment of TGF- $\beta 3$ plus BMP7 greatly promoted cell proliferation in pellets in normoxia 


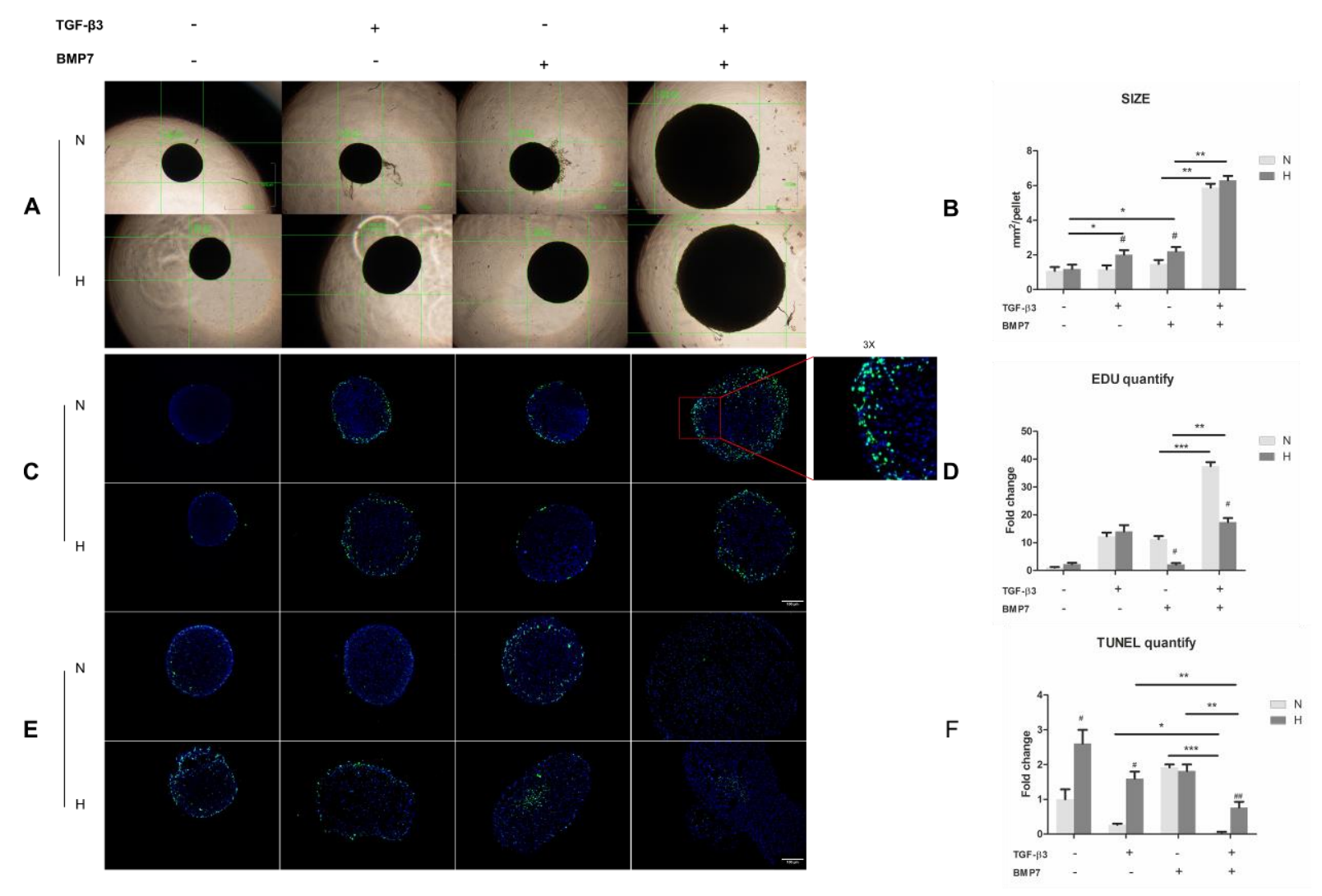

Figure 2 Cell proliferation and apoptosis in OA pellets. A. Pellets imaged by light microscopy. Scare bar $=1000 \mu \mathrm{m}$. B. Measurement of pellet size. Data represents the mean of three pellets in each group. \# indicates a significant difference between normoxia and hypoxia; * represents $\mathrm{P}<0.05$. ** represents $\mathrm{P}<0.01$.*** represents $\mathrm{P}<0.001$. Error bar represents Standard Deviation (S. D.). C. EdU staining of pellets at day 21. EdU incorporation into newly synthesized DNA was visualized by Alexa 488 (green).

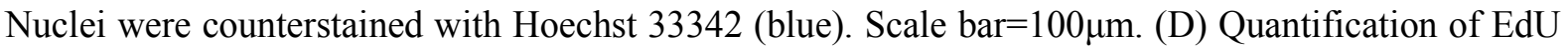
positive chondrocytes. N, normoxia; H, hypoxia; \# indicates a significant difference between normoxia and hypoxia; * represents $\mathrm{P}<0.05$. ** represents $\mathrm{P}<0.01$.*** represents $\mathrm{P}<0.001$. Error bar represents Standard Deviation (S. D.).

while only a modest increase was found in hypoxia, compared to the TGF- $\beta 3$ or BMP7 treated groups. BMP7 treated pellets showed more apoptotic cells in normoxia and hypoxia. TGF- $\beta 3$ increased the number of apoptotic cells in hypoxia only. Remarkably, co-treatment effectively decreased the number of apoptotic cells in hypoxia (Figure 2E and 2F). Alcian blue (Figure $3 \mathrm{~A}$ ) and safranin O (Figure 3B) showed that TGF- $\beta 3$ or BMP7 treatment dramatically enhanced GAG production compared to control in hypoxia while this effect was not observed in normoxia. However, co-stimulation of TGF- $\beta 3$ and BMP7 significantly increased GAG deposition both in normoxia and hypoxia. GAG assay confirmed these results (Figure 3C). 


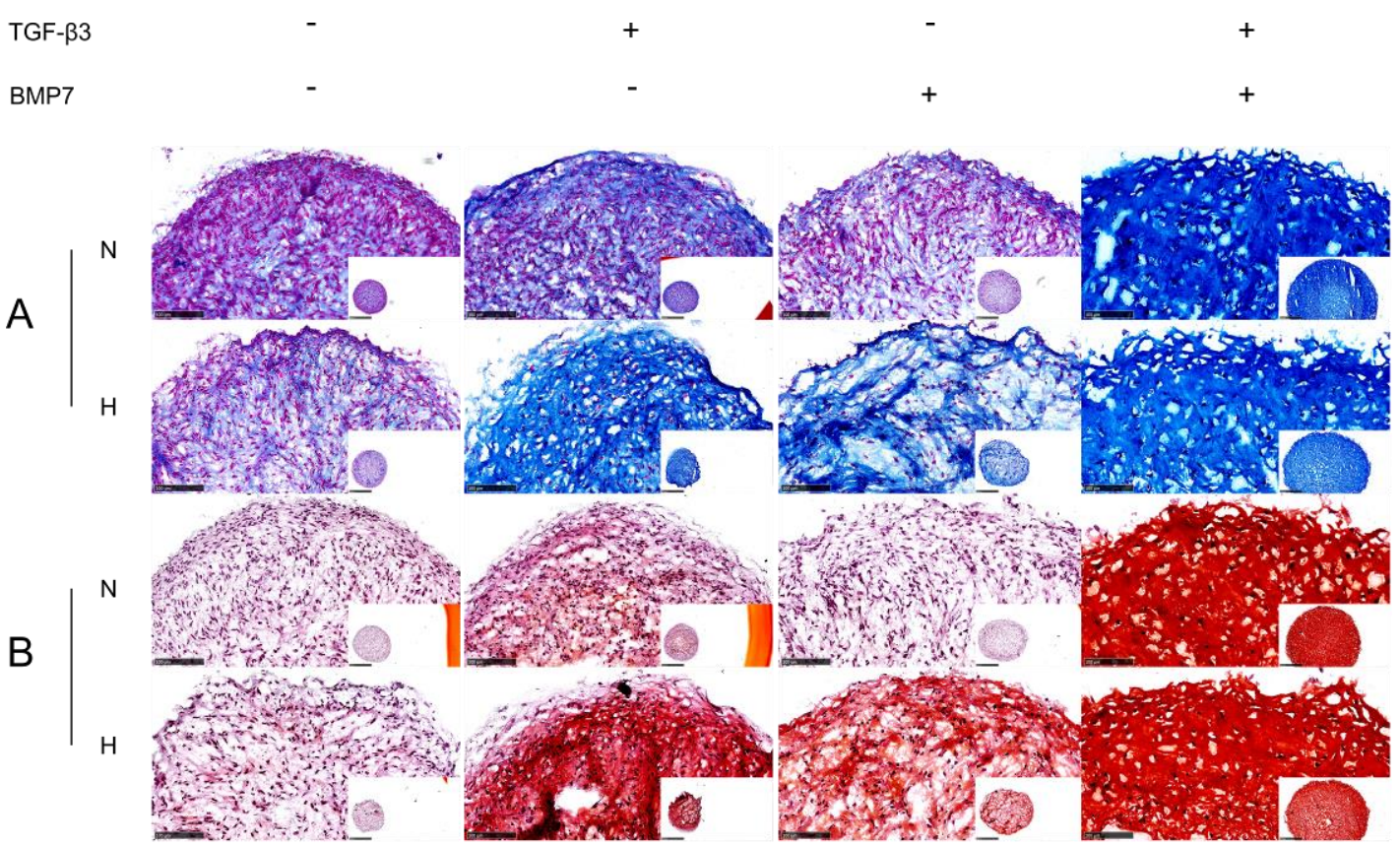

C
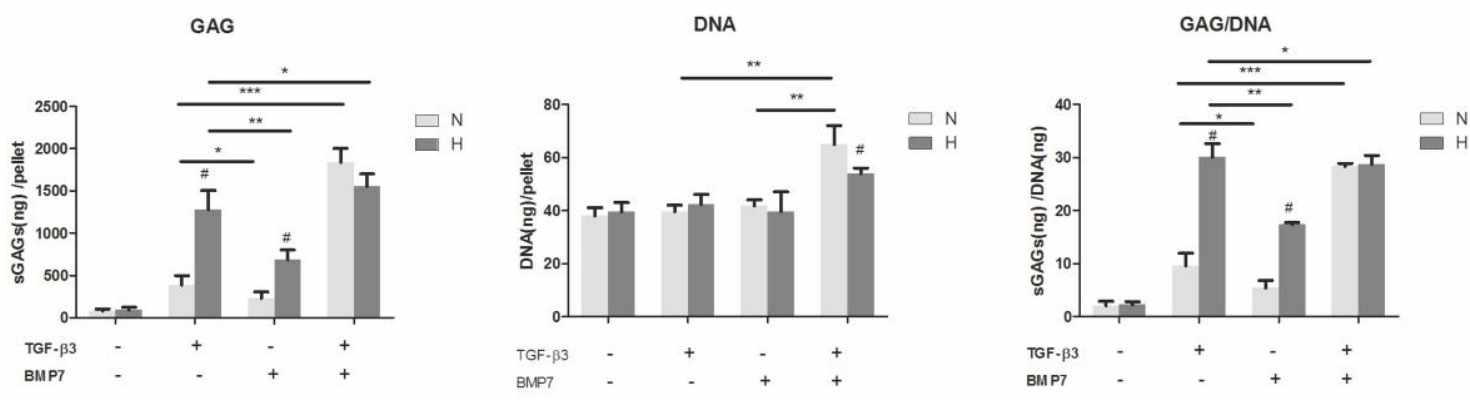

Figure 3 GAG production in pellets. A. Alcian blue staining, nuclei were counterstained by nuclear fast red; B. Safranin O staining, nuclei were counterstained by haematoxylin; C. GAG and DNA assay. $\mathrm{N}$, normoxia; $\mathrm{H}$, hypoxia; * $\mathrm{p}<0.05$; ** $\mathrm{p}<0.01$; \# represents a significant difference between normoxia and hypoxia $(\mathrm{p}<0.05)$.

Co-stimulation of TGF- $\beta 3$ and BMP7 strongly increased GAG production compared to the TGF- $\beta 3$ or BMP7 treated groups both in normoxia and hypoxia.

Anabolic and catabolic gene expression profiles of OA chondrocytes treated with TGF- $\beta 3$ and/or BMP7

Next, we measured the COL2A1 expression and distribution by immunofluorescence. Chondrocyte pellets treated with BMP7 or TGF- $\beta 3$ alone increased COL2A1 expression 
compared to control (Figure 4A) in normoxia. The most pronounced effect was found in the co-treatment group. In hypoxia, TGF- $\beta 3$ was more effective in increasing COL2A1 expression than BMP7. Co-treatment did not have an additive effect.

In order to measure MMP1 secretion, medium was collected every week and measured by ELISA. As Figure 4B shows, the expression of MMP1 in culture medium gradually decreased in the order of control $>$ TGF- $\beta 3>$ BMP7 $>$ TGF- $\beta 3+$ BMP7 in normoxia. In hypoxia, the same trend was observed with the exception that MMP1 expression was relatively higher in the first week compared to normoxia.

A

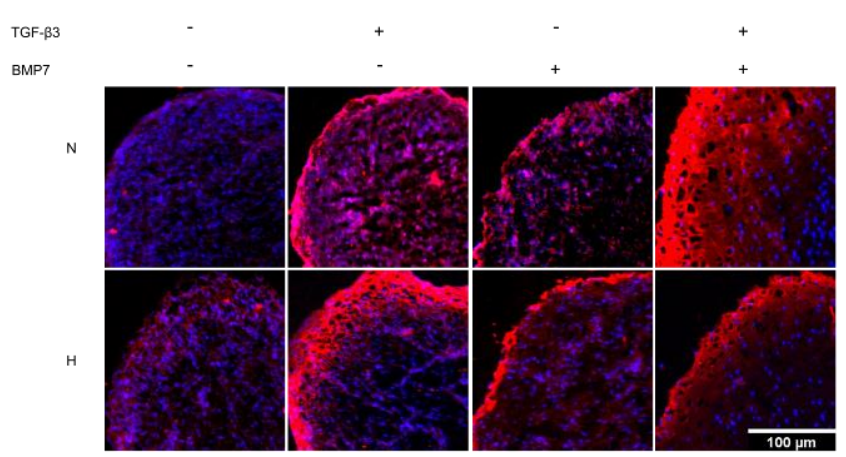

C

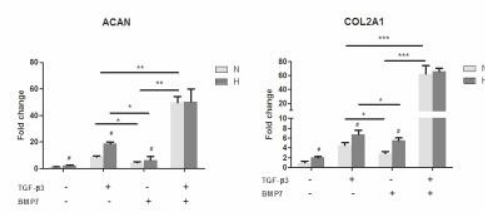

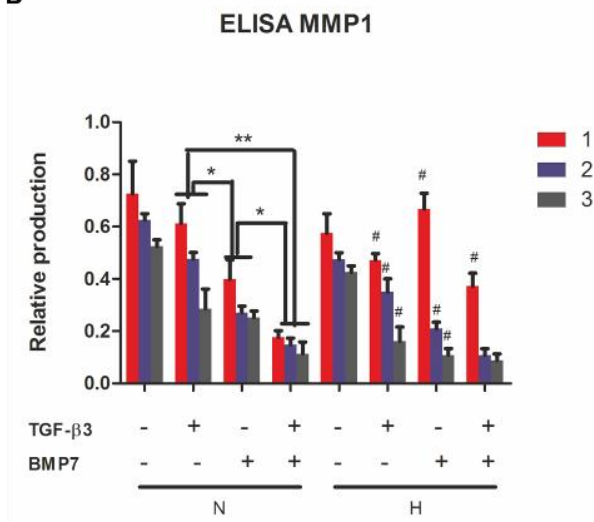

Figure 4 The anabolic and catabolic gene expression profiles of $\mathrm{OA}$ chondrocytes after treatment with TGF- $\beta 3$ and/or BMP7 in normoxia and hypoxia. A. Measurement of COL2A1 expression by immunofluorescence. COL2A1 was detected by mouse anti-COL2A1 antibody, followed by antimouse secondary antibody coupled to Alex 564. Fluorescent images were taken by BD pathway confocal microscopy. Cell nuclei were counterstained with DAPI. Scare bar $=100 \mu \mathrm{m}$. B. Secreted MMP1 was measured by ELISA. C. Gene expression of ACAN, COL2A1, COL1A1, MMP1, 3, 13 was measured by Q-PCR. N, normoxia; H, hypoxia; * $\mathrm{p}<0.05$; ** $\mathrm{p}<0.01$; \# indicates a significant difference with corresponding ones between normoxia and hypoxia $(\mathrm{p}<0.05)$.

The anabolic and catabolic gene expression profiles were measured by Q-PCR. The cartilage markers $A C A N$ and $C O L 2 A 1$ showed similar expression trends. Pellets with TGF- $\beta 3$ treatment showed higher expression than BMP7 treatment. Co-treatment of BMP7 and TGF- $\beta 3$ showed 
highest expression of $A C A N$ and COL2Al both in normoxia and hypoxia. The expression of $A C A N$ and COL2A1 is higher in hypoxia than normoxia irrespective of treatment with either TGF- $\beta 3$ or BMP7. Catabolic genes such as MMP1, 3, 13 and dedifferentiation marker COL1A1 showed opposite trends compared to the anabolic genes. Their expression gradually decreased in the order of TGF- $\beta 3>$ BMP7 $>$ TGF- $\beta 3+$ BMP7 both in normoxia and hypoxia. The combined group of BMP7 and TGF- $\beta 3$ showed the lowest catabolic gene expression compared to single treatments. Catabolic gene expression in normoxia was higher than in the counter treatments cultured in hypoxia. This effect was less pronounced in the TGF- $\beta 3$ and BMP7 cotreatment groups.

\section{SMAD 1/5/8 signaling in OA chondrocytes with treatment of TGF- $\beta 3$ and BMP7}

We next examined the translocation of SMAD 1/5/8 to the nucleus upon treatment with TGF$\beta 3$, BMP7 or in co-treatment in normoxia and hypoxia. The strongest positive staining was observed in the co-treatment group compared to the single treatments both in normoxia and hypoxia (Figure 5). Stronger positive nuclear staining of SMAD 1/5/8 was observed in hypoxia than in normoxia.

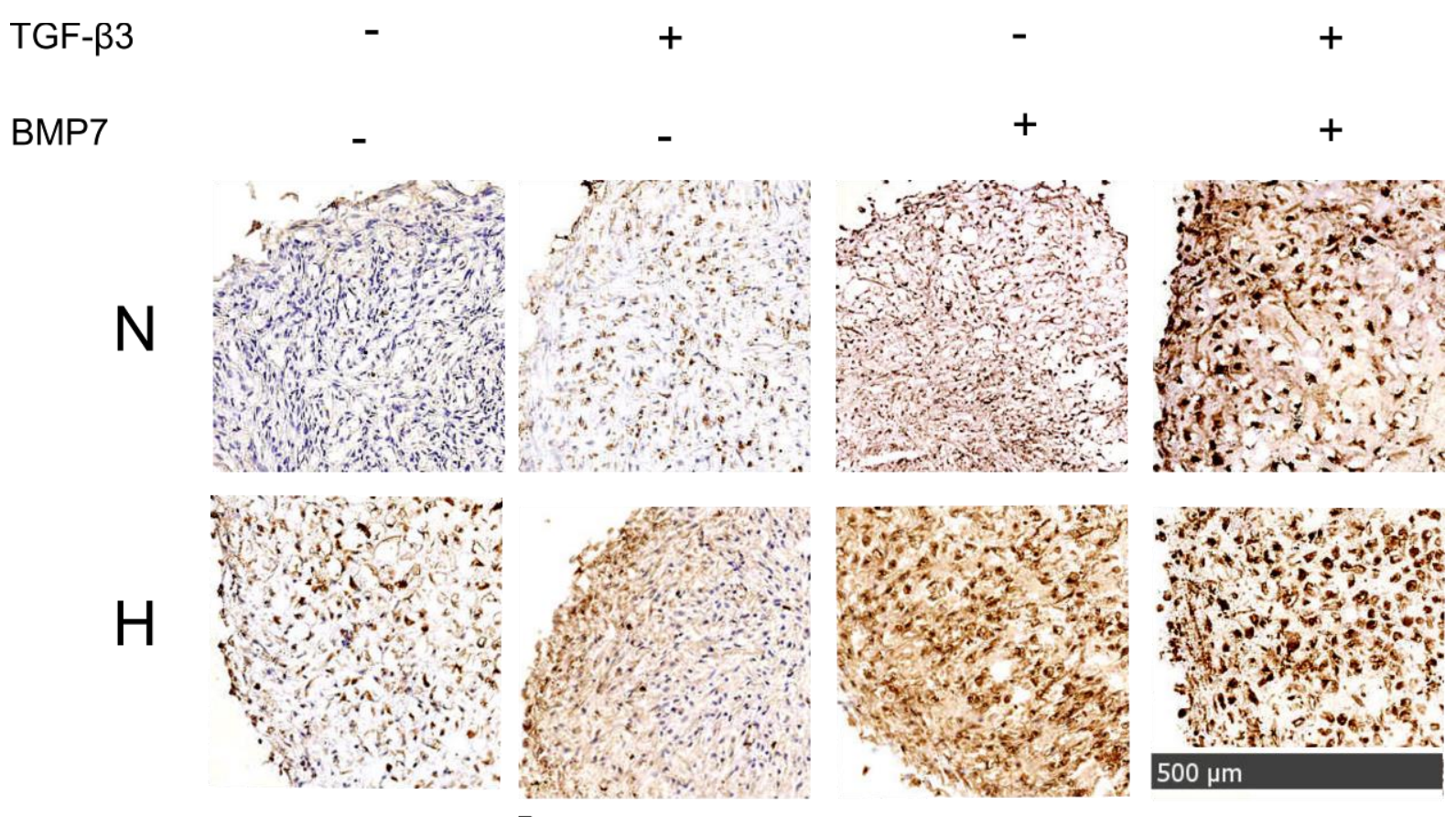

Figure 5 The expression of SMAD 1/5/8 in pellets in normoxia and hypoxia after treatment with TGF- $\beta 3$ and BMP7. Protein expression of SMAD 1/5/8 was detected by mouse anti-SMAD 1/5/8 
primary antibody, followed by an biotinylated anti-mouse secondary antibody followed by signal development using Horse Radish Peroxidase-Streptavidin. For visualization, DAB substrate kit was used. Images were taken by Nanozoomer. Nuclei were counterstained with haematoxylin. Scale bar $=$ $500 \mu \mathrm{m}$. N, normoxia; $\mathrm{H}$, hypoxia.

\section{Discussion}

Although intra-articular injection of BMP7 has been evaluated in clinical trials for the treatment of OA, only few studies have focused on the expression and role of endogenous BMP7 in human cartilage. To the best of our knowledge, this study for the first time has evaluated endogenous BMP7 expression and its intracellular signaling target SMAD 1/5/8 in different grades of OA in human cartilage by immunohistochemistry. We show that BMP7 and SMAD1/5/8 are predominantly present inside cells and in the surrounding pericellular matrix of chondrocytes in the superficial and upper middle layers, and in chondrocytes of the transitional layer. Their expression initially decreased in early OA from grade 0 to 3 and then started to increase again from grade 3 to 5. At grade 5, BMP7 was especially highly expressed in chondrocyte clusters. Bobinac and his colleagues also found that BMP7 is strongly expressed in chondrocyte clusters [42]. Given the anabolic role of BMP7 in cartilage homeostasis, increased expression in chondrocyte clusters may be considered as a reparative response of the tissue. The expression of SMAD 1/5/8 followed the pattern of BMP7 expression albeit at a lower level.

Chondrocytes are the only cell type in cartilage, and are consequently responsible for maintaining the surrounding matrix by synthesizing cartilage specific matrix proteins such as type II collagen and aggrecan. Autologous Chondrocyte Implantation (ACI) has proven to be a pivotal approach as restorative cartilage surgery. However, even the most recent ACI techniques have some limitations. It has been shown that the transplanted chondrocytes are able to form new cartilage, which appears to be of hyaline nature and expresses typical cartilage markers such as type II collagen, aggrecan and COMPs. However type I collagen and fibrous cartilage matrix are also detected in the newly formed tissue after two years [43, 44]. There are plenty of cytokines and growth factors in the cartilage or synovial fluid to modulate the balance between the synthesis and degradation of ECM, or the balance between anabolism and catabolism. BMP/TGF- $\beta$ signaling is extremely important in chondrocyte development. TGF$\beta$ induces collagen II and SOX9 deposition through SMAD2/3 phosphorylation pathway, while BMP7 promotes chondrocyte matrix production and inhibits chondrocyte hypertrophy via 
SMAD1/5/8 phosphorylation. The balance between SMAD 2/3 and SMAD 1/5/8 signaling, which are activated by TGF- $\beta$ and BMP7 play a key role in cartilage hemostasis [45-47]. During ACI surgery, chondrocytes isolated from healthy looking area in cartilage may have limited matrix production capacity and the expansion process further alleviate this potential. Therefore, efficient redifferentiation is a prerequisite before or after implant into damaged area to enable effective cartilage repair.

BMP7 and TGF- $\beta$ are important in the maintenance of cartilage homeostasis, and the loss of their expression could contribute to the development of OA. In this research, we mainly focus on the function of BMP7 and TGF- $\beta$ in chondrocyte redifferentiation in 3D pellet cultures after expanding in monolayer, in serum free chondrogenic differentiation medium supplemented by TGF- $\beta 3$ or BMP7 or both. Cartilage is exposed to a low oxygen environment in vivo. Despite this, chondrocytes are still cultured under normoxia in most of labs. To study the effect of oxygen tension on the chondrocyte's response to these growth factors we compared the effects of growth factors on chondrocyte redifferentiation in both hypoxia $\left(2.5 \% \mathrm{O}_{2}\right)$ and normoxia $\left(21 \% \mathrm{O}_{2}\right)$. Our results indicated that TGF- $\beta 3$ or BMP7 alone were not as efficient in redifferentiating chondrocytes as compared to the combined treatment with TGF- $\beta 3$ and BMP7 both in normoxia and hypoxia. Alcian blue and Safranin O staining, GAG assay, COL2A1 staining and gene expression analysis showed the same trend that the combined treatment with TGF- $\beta 3$ and BMP-7 positively influenced redifferentiation of OA chondrocytes. Previously it has been reported that BMP7 inhibited hMSC growth in expantion cultures [35]. We observed that the combination of BMP7 and TGF- $\beta 3$ significantly promoted cell proliferation. However, the observed enlarged pellet size was mainly due to more extracellular matrix production, with less tightly packed cells in the pellets than other treatments.

In hypoxia, MMP1 expression was relatively higher in the treatment of BMP7 alone or combined with TGF- $\beta 3$ and lower in the TGF- $\beta 3$ alone treatment in the first week compared to normoxia, which indicate chondrocytes are more sensitive under hypoxia for the BMP7 stimulation in the early stage of redifferentiation. Compared to normoxia, anabolic gene expression was higher in hypoxia with the exception of the co-treatment groups. In this condition, anabolic gene expression was comparable in hypoxia and normoxia. Higher staining of SMAD 1/5/8 was observed under hypoxia than normoxia in all treatments and much higher when BMP7 was present, highest expression was noted in the combined treatment of BMP7 and TGF- $\beta 3$ in hypoxia. However, Lafont and his colleagues reported that hypoxia inhibit the 
BMP2 induced smad signaling in the monolayer cultures of human articular chondrocytes [48]. These phenomenons may indicate that hypoxia modulate the sensitivity of the chondrocytes for BMP signaling.

The mechanism of the additive effects of BMP-7 and TGF- $\beta 3$ on chondrocyte redifferentiation and the influence of oxygen tension on this mechanism is still not clear. It might be that TGF$\beta 3$ upregulates the BMP receptors in chondrocytes, which in turn promotes the cellular response to BMP-7. It has been reported that the TGF- $\beta 3$ mediates the upregulation of the type I BMP receptor, ALK6 through which BMP-7 and other BMPs are recruited to stimulate signal transduction in human MSC [49]. Furthermore, it has been reported that the TGF- $\beta 3$ receptor can also function as a BMP receptor by binding multiple members of the BMP subfamily including BMP-7 [50]. The mutual regulation or receptor activation may amplify and enhance the effects of TGF- $\beta 3$ and BMP7.

A

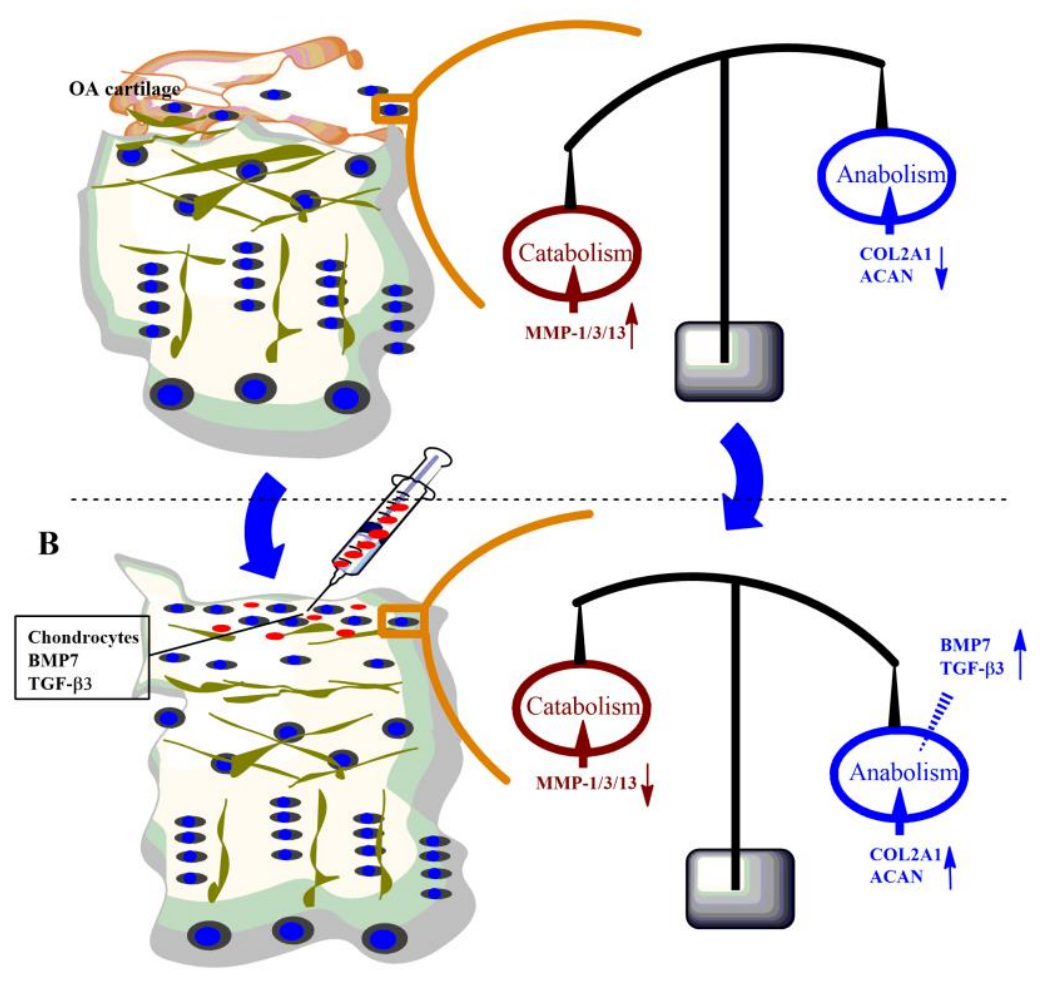

Figure 6 The schematic diagram of OA treatment by TGF-ß3 and BMP7. A Osteoarthritic cartilage with a degenerative superficial layer and loss of chondrocytes shows higher catabolism, reflecting by 
elevated MMP-1/3/13 expression; B After treatment by the combination of TGF- $\beta 3$ and BMP7, superficial layer with gained energetic chondrocytes may exhibit higher anabolism, reflecting by increased expression of COL2A1, ACAN.

To the best of our knowledge, this is the first study using human OA chondrocyte to study the potential application of BMP7 and TGF- $\beta$ in cartilage tissue regeneration in 3D-cultured pellets under hypoxia. As Figure 6 shows, the combination of TGF- $\beta 3$ and BMP-7 might readjust the balance between catabolism and anabolism by upregulating anabolic activities and downregulating catabolic activities in the treatment of osteoarthritic cartilage. Taken together, the combined treatment of BMP7 and TGF- $\beta 3$ was superior in chondrocyte redifferentiation than the single treatments both in normoxia and hypoxia. This study also indicated that a balanced combination of cytokines or growth factors in combination with an appropriate oxygen tension is required for efficient chondrocyte redifferentiation in pellet cultures. 


\section{References}

1. Brittberg, M., et al., Treatment of deep cartilage defects in the knee with autologous chondrocyte transplantation. N Engl J Med, 1994. 331(14): p. 889-95.

2. Cournil-Henrionnet, C., et al., Phenotypic analysis of cell surface markers and gene expression of human mesenchymal stem cells and chondrocytes during monolayer expansion. Biorheology, 2008. 45(3-4): p. 513-26.

3. Hubka, K.M., et al., Enhancing chondrogenic phenotype for cartilage tissue engineering: monoculture and coculture of articular chondrocytes and mesenchymal stem cells. Tissue Eng Part B Rev, 2014. 20(6): p. 641-54.

4. Peterson, L., et al., Two- to 9-year outcome after autologous chondrocyte transplantation of the knee. Clin Orthop Relat Res, 2000(374): p. 212-34.

5. Roberts, S., et al., Autologous chondrocyte implantation for cartilage repair: monitoring its success by magnetic resonance imaging and histology. Arthritis Res Ther, 2003. 5(1): p. R60-73.

6. Yang, X., et al., TGF-beta/Smad3 signals repress chondrocyte hypertrophic differentiation and are required for maintaining articular cartilage. J Cell Biol, 2001. 153(1): p. 35-46.

7. Li, T.F., et al., Smad3-deficient chondrocytes have enhanced BMP signaling and accelerated differentiation. J Bone Miner Res, 2006. 21(1): p. 4-16.

8. Furumatsu, T., et al., Smad3 induces chondrogenesis through the activation of SOX9 via CREB-binding protein/p300 recruitment. J Biol Chem, 2005. 280(9): p. 8343-50.

9. Kang, J.S., et al., Repression of Runx2 function by TGF-beta through recruitment of class II histone deacetylases by Smad3. EMBO J, 2005. 24(14): p. 2543-55.

10. Hellingman, C.A., et al., Smad signaling determines chondrogenic differentiation of bone-marrow-derived mesenchymal stem cells: inhibition of Smad1/5/8P prevents terminal differentiation and calcification. Tissue Eng Part A, 2011. 17(7-8): p. 1157-67.

11. Barry, F., et al., Chondrogenic differentiation of mesenchymal stem cells from bone marrow: differentiation-dependent gene expression of matrix components. Exp Cell Res, 2001. 268(2): p. 189-200.

12. Weiss, S., et al., Impact of growth factors and PTHrP on early and late chondrogenic differentiation of human mesenchymal stem cells. J Cell Physiol, 2010. 223(1): p. 8493.

13. Song, J.J., et al., Connective tissue growth factor (CTGF) acts as a downstream 
mediator of TGF-betal to induce mesenchymal cell condensation. J Cell Physiol, 2007. 210(2): p. 398-410.

14. Tuli, R., et al., Transforming growth factor-beta-mediated chondrogenesis of human mesenchymal progenitor cells involves $N$-cadherin and mitogen-activated protein kinase and Wnt signaling cross-talk. J Biol Chem, 2003. 278(42): p. 41227-36.

15. Ballock, R.T., et al., TGF-beta 1 prevents hypertrophy of epiphyseal chondrocytes: regulation of gene expression for cartilage matrix proteins and metalloproteases. Dev Biol, 1993. 158(2): p. 414-29.

16. Tschan, T., et al., Autocrine or paracrine transforming growth factor-beta modulates the phenotype of chick embryo sternal chondrocytes in serum-free agarose culture. J Biol Chem, 1993. 268(7): p. 5156-61.

17. Dieudonne, S.C., et al., Opposite effects of osteogenic protein and transforming growth factor beta on chondrogenesis in cultured long bone rudiments. J Bone Miner Res, 1994. 9(6): p. 771-80.

18. Bohme, K., K.H. Winterhalter, and P. Bruckner, Terminal differentiation of chondrocytes in culture is a spontaneous process and is arrested by transforming growth factor-beta 2 and basic fibroblast growth factor in synergy. Exp Cell Res, 1995. 216(1): p. 191-8.

19. Narcisi, R., et al., TGF beta-1 administration during ex vivo expansion of human articular chondrocytes in a serum-free medium redirects the cell phenotype toward hypertrophy. J Cell Physiol, 2012. 227(9): p. 3282-90.

20. Shen, J., S. Li, and D. Chen, TGF-beta signaling and the development of osteoarthritis. Bone Res, 2014. 2.

21. van der Kraan, P.M., et al., TGF-beta signaling in chondrocyte terminal differentiation and osteoarthritis: modulation and integration of signaling pathways through receptorSmads. Osteoarthritis Cartilage, 2009. 17(12): p. 1539-45.

22. Chen, D., M. Zhao, and G.R. Mundy, Bone morphogenetic proteins. Growth Factors, 2004. 22(4): p. 233-41.

23. Zhang, M., et al., BMP-2 modulates beta-catenin signaling through stimulation of Lrp5 expression and inhibition of beta-TrCP expression in osteoblasts. J Cell Biochem, 2009. 108(4): p. 896-905.

24. Retting, K.N., et al., BMP canonical Smad signaling through Smad1 and Smad5 is required for endochondral bone formation. Development, 2009. 136(7): p. 1093-104.

25. Miyazono, K., S. Maeda, and T. Imamura, Coordinate regulation of cell growth and 
differentiation by TGF-beta superfamily and Runx proteins. Oncogene, 2004. 23(24): p. $4232-7$.

26. Leboy, P., et al., Smad-Runx interactions during chondrocyte maturation. J Bone Joint Surg Am, 2001. 83-A Suppl 1(Pt 1): p. S15-22.

27. Caron, M.M., et al., Hypertrophic differentiation during chondrogenic differentiation of progenitor cells is stimulated by BMP-2 but suppressed by BMP-7. Osteoarthritis Cartilage, 2013. 21(4): p. 604-13.

28. Haaijman, A., et al., Inhibition of terminal chondrocyte differentiation by bone morphogenetic protein 7 (OP-1) in vitro depends on the periarticular region but is independent of parathyroid hormone-related peptide. Bone, 1999. 25(4): p. 397-404.

29. Gautschi, O.P., S.P. Frey, and R. Zellweger, Bone morphogenetic proteins in clinical applications. ANZ J Surg, 2007. 77(8): p. 626-31.

30. Shintani, N., K.A. Siebenrock, and E.B. Hunziker, TGF-ss1 enhances the BMP-2induced chondrogenesis of bovine synovial explants and arrests downstream differentiation at an early stage of hypertrophy. PLoS One, 2013. 8(1): p. e53086.

31. Toh, W.S., et al., Combined effects of TGFbetal and BMP2 in serum-free chondrogenic differentiation of mesenchymal stem cells induced hyaline-like cartilage formation. Growth Factors, 2005. 23(4): p. 313-21.

32. Shen, B., et al., BMP-2 enhances TGF-beta3-mediated chondrogenic differentiation of human bone marrow multipotent mesenchymal stromal cells in alginate bead culture. Tissue Eng Part A, 2009. 15(6): p. 1311-20.

33. Nakayama, N., et al., Macroscopic cartilage formation with embryonic stem-cellderived mesodermal progenitor cells. J Cell Sci, 2003. 116(Pt 10): p. 2015-28.

34. Miyamoto, C., et al., Osteogenic protein-1 with transforming growth factor-beta1: potent inducer of chondrogenesis of synovial mesenchymal stem cells in vitro. J Orthop Sci, 2007. 12(6): p. 555-61.

35. Shen, B., et al., The role of BMP-7 in chondrogenic and osteogenic differentiation of human bone marrow multipotent mesenchymal stromal cells in vitro. J Cell Biochem, 2010. 109(2): p. 406-16.

36. Silver, I.A., Measurement of $\mathrm{pH}$ and ionic composition of pericellular sites. Philos Trans R Soc Lond B Biol Sci, 1975. 271(912): p. 261-72.

37. Sheehy, E.J., C.T. Buckley, and D.J. Kelly, Oxygen tension regulates the osteogenic, chondrogenic and endochondral phenotype of bone marrow derived mesenchymal stem cells. Biochem Biophys Res Commun, 2012. 417(1): p. 305-10. 
38. Hirao, M., et al., Oxygen tension regulates chondrocyte differentiation and function during endochondral ossification. J Biol Chem, 2006. 281(41): p. 31079-92.

39. Zhong, L., et al., Correlation between Gene Expression and Osteoarthritis Progression in Human. Int J Mol Sci, 2016. 17(7).

40. Wu, L., et al., Serum regulates adipogenesis of mesenchymal stem cells via MEK/ERKdependent PPARgamma expression and phosphorylation. J Cell Mol Med, 2010. 14(4): p. $922-32$.

41. Leijten, J., et al., Metabolic programming of mesenchymal stromal cells by oxygen tension directs chondrogenic cell fate. Proc Natl Acad Sci U S A, 2014. 111(38): p. 13954-9.

42. Bobinac, D., et al., Expression of bone morphogenetic proteins, cartilage-derived morphogenetic proteins and related receptors in normal and osteoarthritic human articular cartilage. Coll Antropol, 2008. 32 Suppl 2: p. 83-7.

43. Peterson, L., et al., Autologous chondrocyte transplantation. Biomechanics and longterm durability. Am J Sports Med, 2002. 30(1): p. 2-12.

44. Marlovits, S., et al., Cartilage repair: generations of autologous chondrocyte transplantation. Eur J Radiol, 2006. 57(1): p. 24-31.

45. Blaney Davidson, E.N., et al., Increase in ALK1/ALK5 ratio as a cause for elevated MMP-13 expression in osteoarthritis in humans and mice. J Immunol, 2009. 182(12): p. 7937-45.

46. van der Kraan, P.M., E.N. Blaney Davidson, and W.B. van den Berg, A role for agerelated changes in TGFbeta signaling in aberrant chondrocyte differentiation and osteoarthritis. Arthritis Res Ther, 2010. 12(1): p. 201.

47. de Kroon, L.M., et al., Activin Receptor-Like Kinase Receptors ALK5 and ALK1 Are Both Required for TGFbeta-Induced Chondrogenic Differentiation of Human Bone Marrow-Derived Mesenchymal Stem Cells. PLoS One, 2015. 10(12): p. e0146124.

48. Lafont, J.E., et al., Hypoxia potentiates the BMP-2 driven COL2A1 stimulation in human articular chondrocytes via p38 MAPK. Osteoarthritis Cartilage, 2016. 24(5): p. 856-67.

49. Xu, D., et al., Potential involvement of BMP receptor type IB activation in a synergistic effect of chondrogenic promotion between rhTGFbeta 3 and rhGDF5 or rhBMP7 in human mesenchymal stem cells. Growth Factors, 2006. 24(4): p. 268-78.

50. Kirkbride, K.C., et al., Bone morphogenetic proteins signal through the transforming growth factor-beta type III receptor. J Biol Chem, 2008. 283(12): p. 7628-37. 
Supplemental Figure 1. The protein expression of BMP7 was detected by IHC in each donor (scale bar: $500 \mu \mathrm{m})$. Images were taken using the Nanozoomer. G0, G1, G2, G3, G4, G5 = Grade 0, Grade 1, Grade 2, Grade 3, Grade 4, Grade 5 osteoarthritic cartilage.

G0
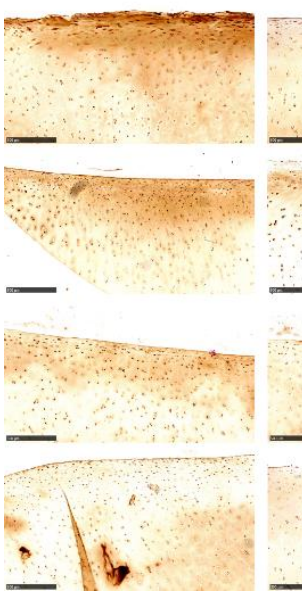
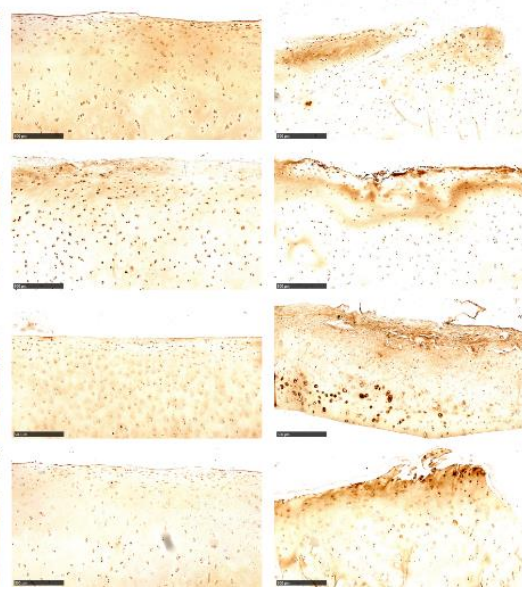

G4

G5
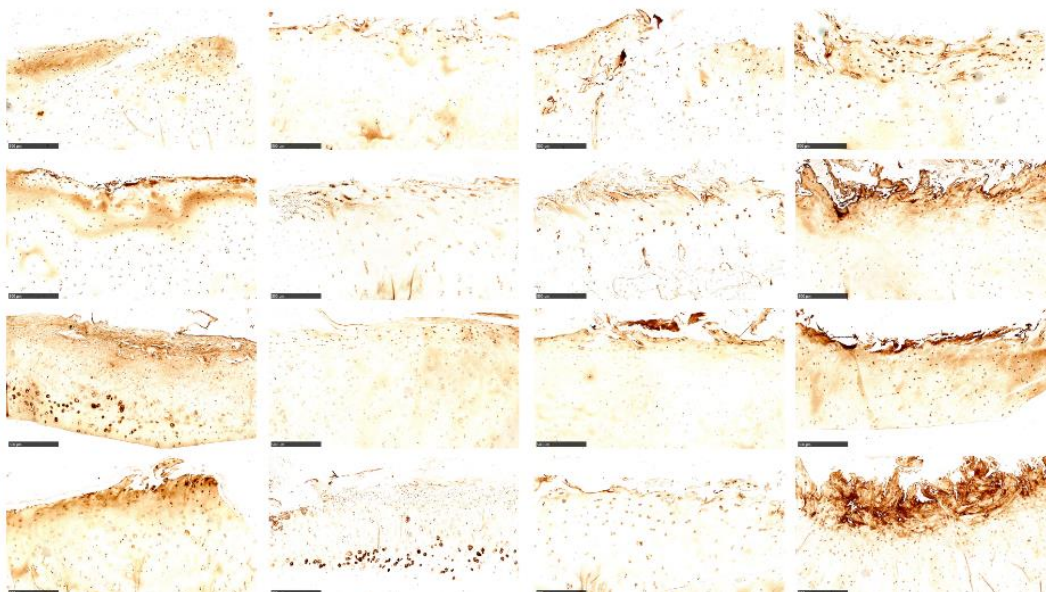

Supplemental Figure 2. The protein expression of SMAD 1/5/8 was detected by IHC in each donor (scale bar: $\mathbf{5 0 0} \boldsymbol{\mu m}$ ). Images were taken using the Nanozoomer. G0, G1, G2, G3, G4, G5 = Grade 0, Grade 1, Grade 2, Grade 3, Grade 4, Grade 5 osteorthritic cartilage.
G0
G1
G3
G4
G5

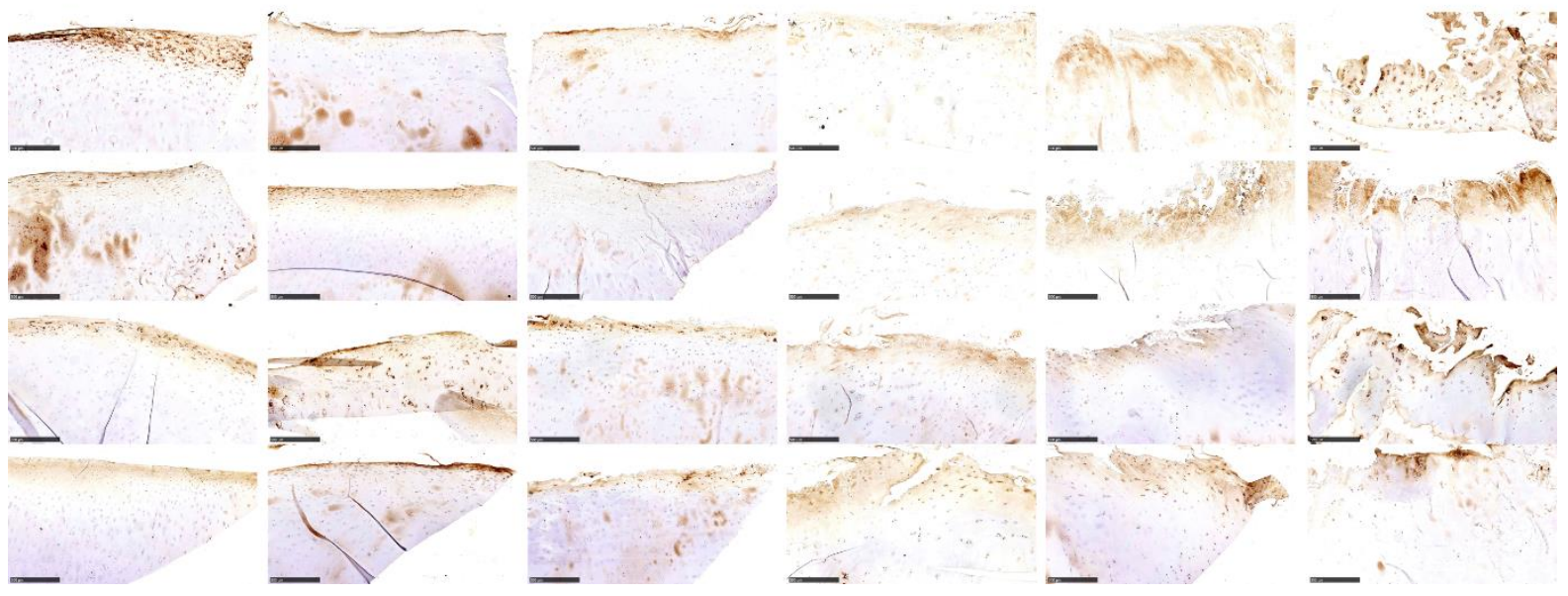




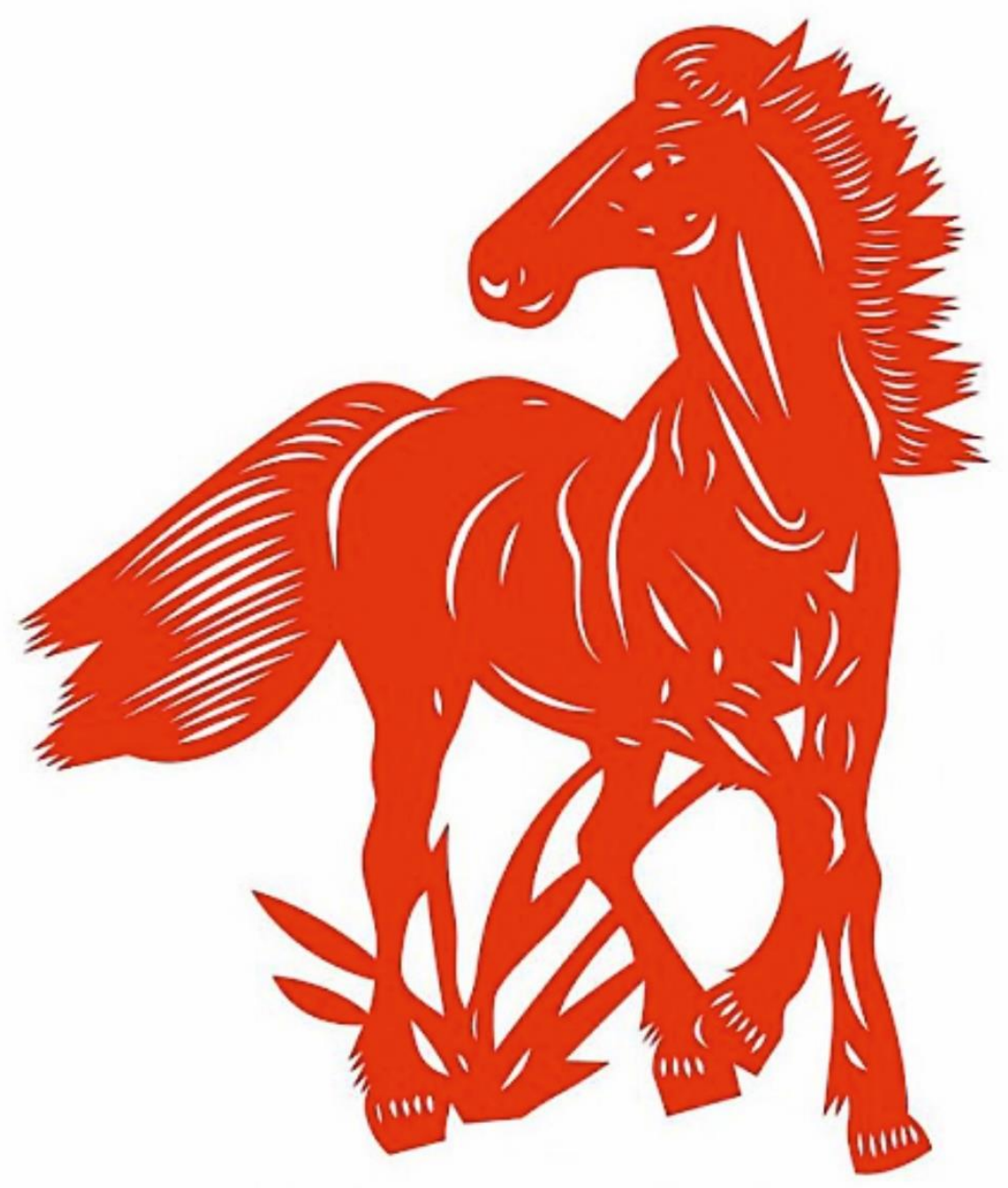




\section{Chapter 7}

\section{Bifunctional VHHs efficiently target hBMP7 to bone after intravenous injection}

Xiaobin Huang ${ }^{1}$, Emilie Dooms Rodrigues ${ }^{1}$, Jasper van Weerd ${ }^{1}$, Leilei Zhong ${ }^{1}$, Janine N. Post ${ }^{1}$, Jeroen C.H. Leijten ${ }^{1}$, Mohamed El Khattabi ${ }^{2}$, Ivo Que ${ }^{3}$, Theo Verrips ${ }^{2}$ and Marcel Karperien ${ }^{1}$

1 Department of Developmental BioEngineering, MIRA Institute for Biomedical Technology and Technical Medicine, University of Twente, Enschede, The Netherlands;

2 QVQ BV, Utrecht, The Netherlands;

3 Department of Radiology, Leiden University Medical Centre, the Netherlands.

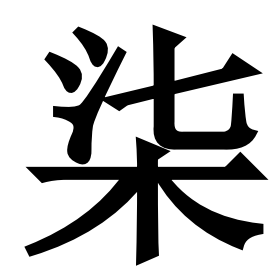

天下万物生于有，有生于无。

For though all creatures under heaven are the products of Being.

Being itself is the product of Not-being. 


\section{Abstract}

In the field of tissue engineering, growth factors have been used as therapeutic agents to stimulate tissue repair and potentially tissue regeneration. Much effort has been put into the development of bioactive scaffolds with controlled growth factor delivery. However, there are some limitations to this approach. In this research, we present the selection of highly specific variable domain of heavy chain only antibodies of Camelidae ( $\mathrm{VHH}$ ) against bone morphogenetic protein 7 (BMP-7) and hydroxyapatite (HA). These two VHHs were genetically fused in a bispecific VHH or bihead using a 10 amino acid glycine linker. The biological function of the bihead was tested in $\mathrm{C} 2 \mathrm{C} 12$ mouse myoblast cells. Bihead labeled with nearinfrared fluorophore IRDye800CW (G7-MA10-IR) mixed with BMP7 was injected intravenously into nude mice. Immunohistochemistry was used to evaluate the specific targeting and signal transduction. We have successfully generated bi-functional biheads binding BMP7 and HA simultaneously. The bihead specically targets to mineralized bone upon intravenous injection and remains present in bone for at least 3 weeks. Upon co-injection, the bihead enables specific targeting of BMP7 to skeletal tissue. Even 3 weeks after injection of the bihead a repeated injection with BMP7 only results in accumulation of this growth factor in the skeleton demonstrating that the biological activity of the bihead remains preserved. These results demonstrate that bispecific VHHs represent a promising strategy for the targeted delivery of growth factors to bone tissue and potentially can be used for the biofunctionalization of scaffolds in tissue engineering without modification of the growth factor.

Keywords: Bihead, VHH, BMP7, Hydroxyapatite, Bone, Targeted drug delivery, Tissue engineering 


\section{Introduction}

Tissue engineering is dedicated to the development of biological substitutes for the restoration, maintenance and improvement of tissue function [1], mainly through three methods: biomaterials or scaffolds, cells and biomolecules (e.g. growth factors) [2-4]. In recent years, much effort has been devoted to the development of smart bioactive scaffolds with controlled growth factor delivery $[5,6]$. Growth factors are critical signaling molecules during cellular and tissue development, capable of triggering specific cellular responses, including cell survival, migration, proliferation, differentiation and adhesion $[5,7,8]$. In the field of tissue engineering, growth factors can be used to bio-functionalize biomaterials by coupling, and they have therefore been put forth as therapeutic agents to stimulate tissue repair and potentially tissue regeneration. However, there are limitations to the immobilization of growth factors in biomaterials. For instance, covalent coupling of the growth factors requires specific coupling sites, which can be difficult to assign, and the growth factors might lose their bioactivity during immobilization due to damage or inaccessibility of the bioactive functional groups [5]. A possible solution to this problem would be to anchor growth factors to the biomaterial by means of antibodies, using high-affinity binders that do not interfere with the active site of the growth factor. Antibodies can bind to growth factors with high specificity and without chemical modifications, and the release of a growth factor from the scaffold surface can be tailored based on the affinity of the antibody for the growth factor [9-11].

After coupling to a biomaterial, antibodies can be loaded with exogenous growth factors or capture locally produced growth factors to create a reservoir of biologically active factors, thereby controlling cell differentiation at the biomaterial implant site. Interactions between antibodies and growth factors can stabilize growth factors and present them to their receptors in order to catalyze downstream signaling pathways. However, conventional antibodies are complex, large- size molecules (they are approximately $160 \mathrm{kDa}$ ), consisting of four protein chains: two heavy chains and two light chains, see Figure 1A. The four chains are connected by disulfide bonds and hydrophobic interactions [11, 12]. However, the use of these 
conventional antibodies to decorate biomaterials and capture growth factors is challenging due to e.g. their large size, complexity and high production costs [13].

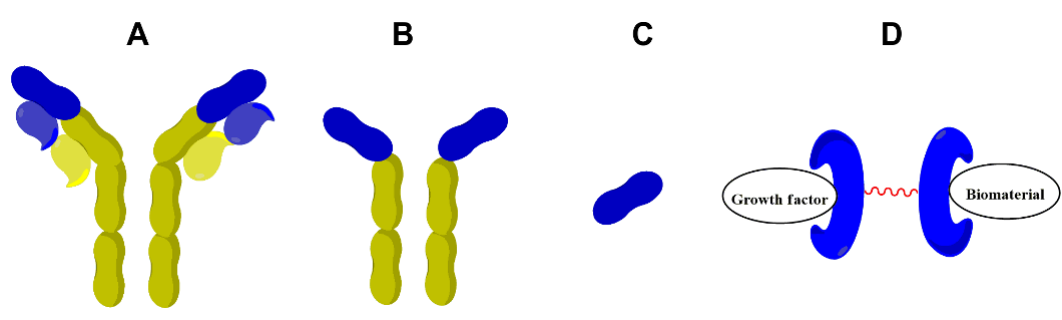

Figure 1. Schematic representation of antibodies. (A) Conventional monoclonal antibody IgG; (B) Heavy chain only antibody; (C) Variable domain of heavy chain of heavy chain only antibody (VHH). Heavy variable domains are represented in blue. (D) Schematic representation of a bivalent VHH as a bridging molecule coupling a growth factor to a biomaterial.

To overcome these issues a special class of antibodies can be used instead, namely the heavy chain only antibodies, Figure 1B. These antibodies are expressed by the Camelidae family (e.g. camels, dromedaries and llamas) [12, 14-19]. They consist of two protein chains only and are devoid of light chains $[14,15,17]$. The antigen-binding domain of these heavy chain only antibodies is called variable domain of heavy chain of the heavy chain only antibody (VHH), Figure 1C. VHHs are small, intact antibody fragments of around $15 \mathrm{kDa}[12,14,15]$. They are $2.5 \mathrm{~nm}$ in diameter and around $4 \mathrm{~nm}$ high $[12,18,20]$. These VHHs, also referred to as nanobodies, are very stable polypeptides with the full antigen-binding capacity of the original heavy-chain only antibody [12]. VHHs have several advantages over conventional antibodies: 1) VHHs can penetrate tissues more effectively due to their small size [12]; 2) VHHs are able to bind specifically and with high affinity $[14,16,18]$ and can recognize antigenic sites/epitopes (e.g. enzyme active sites) normally not recognized by conventional antibodies $[12,15] ; 3)$ since VHHs consist of only one polypeptide, coded in one gene, they are easy to clone and genetically modify [14]; 4) VHHs can be recombinantly produced in high quantities in yeast and bacteria $[15,18]$ with straightforward affinity purification techniques [14]; 5) VHHs are soluble and do not have a tendency to aggregate [12, 20]; 6) VHHs are relatively heat stable and can remain functional at $90^{\circ} \mathrm{C}$ or after incubation at high temperatures $[12,15$, 
16]; 7) VHHs are expected to be able to stay biologically active under harsh conditions such as those in the stomach or gut $[12,18]$, and: 8) VHHs are resistant to many organic solvents thus ideally suited for use in mild chemical coupling reactions required for biomaterial functionalization.

The use of VHHs in tissue engineering is still largely unexplored. Bispecific antibodies/VHHs (bihead VHHs), which bind to the biomaterial or ECM and the growth factor simultaneously, could have great potential to functionalize biomaterials without loss of the biological activities of growth factor. Hydroxyapatite (HA) is a calcium phosphate biomaterial/ceramic with maximum bio-compatibility, and a naturally occurring structure in bone mineral; $90 \%$ of the inorganic bone matrix consists of HA [21]. It is widely used in bone tissue engineering as a scaffold to substitute bone grafts [2, 21, 22]. Bone morphogenetic protein 7 (BMP-7), also known as Osteogenic protein-1, is part of the BMP family [23]. BMPs, which are known to induce bone and cartilage formation $[3,23]$ and are produced at sites of bone trauma, are already used clinically [3, 24]. However, BMPs have only a short half-life and are instable.

In this research, an ECM binding VHH that was selected against HA and a growth factor binding VHH selected against human BMP7 (hBMP7) were studied. To this end, VHHs that can bind to HA and hBMP7 were identified and characterized. A bi-specific VHH was prepared by genetic fusion of the VHH G7 (binding to BMP-7) and the VHH MA10 (binding to HA) with a GS-linker in between (G7GS10MA10). The biological activity of the bihead VHH was analyzed in C2C12 mouse myoblast cells (as a model of osteogenic differentiation). Bihead labeled with the near-infrared fluorophore IRDye800CW (G7GS10MA10-IR) was mixed with BMP7 and injected intravenously in nude mice to study its use for targeting BMP7 to bone in vivo. 


\section{Materials and methods}

\section{VHHs selection and production}

An overview of the VHH production process is shown in Figure 2.

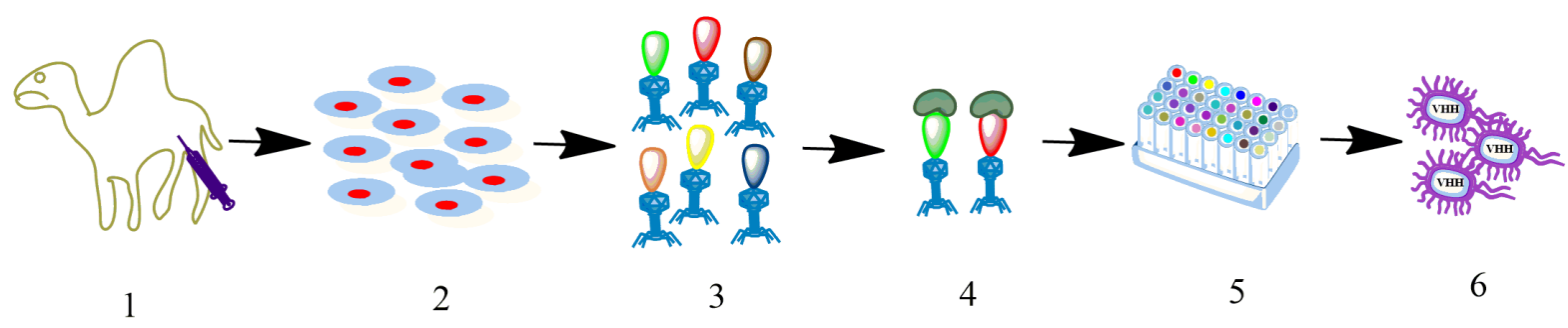

Figure 2. Schematic overview of the selection of VHHs using phage display technology. 1 Immunization of llamas with antigens; 2 collection of lymphocytes and RNA isolation, followed by reverse transcription to obtain cDNA; 3 phage display of a VHH cDNA library cloned into phagemid vector after infection with Escherichia coli (E.coli); 4 selection of a phage that binds to antigen; 5 screening and sequencing of large numbers of VHHs; 6 Isolation and expression of individual VHHs from E.coli or yeast.

\section{1) Library construction and selection of VHH targeting BMP7}

All immunizations were approved by the Utrecht University ethical committee for animal experimentation. Two llamas were immunized with recombinant human BMP7 (R\&D systems, \#354-BP/CF). The antigens were mixed with the adjuvant Stimune (CEDI Diagnostics, Lelystad, the Netherlands) and injected intramuscularly at days 0, 14, 28 and 35. At day 44, peripheral blood lymphocytes were isolated for RNA extraction and library construction. The VHH phage display libraries were generated as previously described [25-27] and transferred to Escherichia coli (E. colli) strain TG1 [supE hsd_5 thi (lac-proAB) F(traD36 proAB_lacIq 
lacZ_M15)] by electroporation.

Phages binding to BMP7 were selected via the panning method. Decreasing concentrations of BMP7 (5 $\mu \mathrm{g}, 2 \mu \mathrm{g}$ and $0.2 \mu \mathrm{g})$ in phosphate buffered saline (PBS, Gibco) were coated in the wells of MaxiSorp plate (Nunc, Thermo Scientific) overnight at $4^{\circ} \mathrm{C}$. Phages, approximately 1010 colony forming units (cfu), were incubated for 2 hours at room temperature (RT) with the coated BMP7, and blocked with 4\% Marvel (dried skimmed milk, Premier International Foods) in PBS. After washing thoroughly, phages binding to BMP7 were eluted by incubating them in $100 \mathrm{mM}$ triethylamine (TEA) for 15 minutes at RT. Eluted phages were immediately neutralized through the addition of $1 \mathrm{M}$ Tris- $\mathrm{HCl}, \mathrm{pH}$ 7.5. DNA information of the selected phages was rescued by means of infection with the E. coli TG1 strain and subsequent selection for ampicillin resistance on agar plates. To obtain recombinant bacteriophages expressing the VHH as fusion proteins with the bacteriophage gene III, rescued TG1 E. coli were grown to logarithmic phase and then infected with helper phage VCSM13 (Stratagene, La Jolla, CA, USA) [28]. The phage particles were precipitated with polyethylene glycol (PEG) and used in the second round of selection, on wells coated with BMP7 as described above. Selected phages from single colonies from the second round of selection were sequenced (Macrogen). Among several VHH candidate clones, the selected one was named VHH G7 (G7) after analyzing for further characterization.

\section{2) Selection of VHH targeting HA}

VHH binding to HA were selected from a non-immunized llama VHH phage library (kindly provided by BAC BV). The construction of the non-immune library has been described before [29]. A two-round selection was performed on HA plates. Pure HA plates measuring approximately $1.5 \times 1.5 \mathrm{~mm}$ and of a $0.1 \mathrm{~mm}$ thickness were supplied by Plasma Biotal Limited, UK. Phages were incubated with 3 HA plates in the wells of a 96-wells microtiter plate. The procedure that followed was the same as for VHH G7 selection. Finally, one VHH, named VHH-MA10 (MA 10), was chosen for further study. 


\section{3) Construction of the bihead VHH G7GS10MA10 recognizing BMP7 and HA}

The bihead G7-MA10 was constructed by genetic fusion of the cDNAs, encoding the VHH binding to BMP7, G7, and the VHH binding to HA, MA10 (Figure 5). PCR was used to amplify the VHH sequences. Different primers sets were designed to amplify the VHH G7, located at the N-terminus, and the VHH MA10, placed at the C-terminus, to generate a bivalent VHH (Table 1). The primers at the 3' of the N-terminus VHH and at the 5' of the C-terminus VHH encoded a flexible sequence (GS linker) represented by the pentapeptide 'Gly-Gly-Gly-GlySer'. The primers contained a unique restriction site (BamHI). After PCR amplification, the generated fragments were digested with a unique $\mathrm{N}$-terminal restriction site (SfiI) and BamHI for the VHH located at the N-terminus, and with BamHI and a unique C-terminal restriction site (BstEII) for VHH located at the C-terminus. The fragments were ligated into the expression vector, pMEK222, which was digested with SfiI and BstEII, after which they were transformed into E.coli for expression.

Table 1. List of sequences of primers used in the bivalent VHH construction.

\begin{tabular}{|l|l|}
\hline Primer & Oligonucleotide sequence \\
\hline M13Rev & GAGGTGCAATTGGTGGAGTCTGGG \\
\hline 5GSBamRev & AGTAGGATCCGCCACCTCCTGAGGAGACCGTGACCTGGGTCCC \\
\hline 5GSBamFwd & TCTTGGATCCGAGGTGCAGCTGGTGGAGTCTGGG \\
\hline TVSSRev & TGAGGAGACGGTGACCTGGGTCCC \\
\hline
\end{tabular}

\section{4) VHH production and purification}

E. coli strain TG1 was used for the maintenance of the plasmids, infection by the phages and expression of proteins. For detection purposes in following assays, two vectors with different tags were used in order to specifically detect the VHH. The DNA information of the individually selected and isolated VHH was therefore subcloned into plasmid pUR8100 (for MA 10) or pMEK219 (for G7) containing a C-terminus Myc and His tags, and pMEK222 containing C-terminus FLAG and His tags. E. coli TG1 was grown in Luria Broth (LB) or yeast 
extract and tryptone (YT) medium containing $2 \%(\mathrm{w} / \mathrm{v})$ glucose and ampicillin at $100 \mu \mathrm{g} / \mathrm{ml}$. VHHs were produced from E. Coli TG1 by Isopropyl $\beta$-D-1-thiogalactopyranoside (IPTG) induction of the lac promoter at $37^{\circ} \mathrm{C}$ for 4 hours under non-static conditions $[15,16]$. VHH proteins were purified from the periplasmic fraction via the C-terminus His-tag by cobalt affinity chromatography (TALON His-Tag Purification Resin, ClonTech). Purified VHHs were analyzed by means of sodium dodecyl sulfate polyacrylamide gel electrophoresis (SDSPAGE). The final VHH concentration was determined on the basis of UV absorption at 280 nm (NanoDrop 1000 Spectrophotometer, Thermo Scientific) and the theoretical mass extinction coefficient.

\section{VHH binding specificity}

The binding specificity of the purified VHHs (G7, MA10, G7-MA10) was tested in an enzyme linked immunosorbent assay (ELISA) binding assay. The binding of MA10 to bone was further tested in mouse fetal metatarsals.

\section{1) Binding specificity of VHH-G7 or bihead G7-MA10 to BMP7}

MaxiSorp plate wells were coated with BMP7 $(30 \mathrm{nM})$ in PBS overnight at $4^{\circ} \mathrm{C}$, after which they were blocked with $2 \%$ BSA in PBS for two hours at RT in order to block non-specific binding sites. Subsequently, wells were incubated with a serial dilution of VHHs (ranging from 0 to $7 \mu \mathrm{M}$ ) in $1 \%$ BSA in PBS for two hours at RT. The wells were washed with PBS tween (PBST) and PBS. Bound VHHs were detected by incubation with a rabbit anti-VHH serum (K976) and a donkey anti-rabbit antibody coupled to a peroxidase. The amount of Horse Raddish Peroxidase (HRP) was developed by the addition of Tetramethylbenzidine (TMB, 1Step Ultra TMB-ELISA, Thermo Scientific). The reactions were stopped by the addition of $\mathrm{H}_{2} \mathrm{SO}_{4}$ and measured at $450 \mathrm{~nm}$ (Micro Plate Reader). The dissociation constants (Kd) were obtained from the subsequent association of experimental data with specific binding models. The data was normalized to the condition without VHHs using Equations 1 and 2. 


$$
\begin{aligned}
& y=A 450-A 450_{(0)} \\
& \text { Binding }[\text { a.u. }]=\frac{y}{\max y} \\
& y=\frac{B \max * x}{K d+x}
\end{aligned}
$$

\section{2) Binding specificity of VHH-MA10 or bihead G7GS10MA10 to HA}

Microtiter plate wells containing three pure HA plates were blocked with 2\% BSA in PBS for two hours at RT in order to block non-specific binding sites. The following steps were performed as G7 to BMP7.

\section{3) VHH-MA10 bind to mouse fetal metatarsals}

Mouse fetal metatarsals were isolated from FVB mouse embryos (time-paired, Harlan) at day 17.5 of gestation [30]. (Animal experiments were approved by a local animal ethical committee). After isolation, metatarsals were individually cultured in wells of 24-well plates in $200 \mu \mathrm{l} \alpha$-MEM, supplemented with $10 \% \mathrm{FBS}, 100 \mathrm{U} / \mathrm{ml}$ penicillin, $100 \mathrm{mg} / \mathrm{ml}$ streptomycin and $1 \%$ Glutamax (Invitrogen) for 48 hours at $37^{\circ} \mathrm{C}$ in humidified atmosphere and $5 \% \mathrm{CO} 2$. After this equilibration period, metatarsals were incubated in the presence of VHH $(1 \mu \mathrm{g} / \mathrm{ml})$ for 2 hours at $37^{\circ} \mathrm{C}$ in humidified atmosphere and $5 \% \mathrm{CO} 2$. To enable visualization, prior to incubation, VHH MA10 and control VHH 1B5 were randomly labeled with Alexa Fluor 647, following the manufacturer's protocol (Invitrogen). This resulted in VHH MA10-647 and 1B5647. After intensive washing with PBS, the metatarsals were evaluated for fluorescence with BD Pathway (BD Biosciences) with an excitation filter of $628 \pm 40 \mathrm{~nm}$ and an emission filter of $692 \pm 40 \mathrm{~nm}$.

\section{4) Dual binding specificity of bihead G7GS10MA10 to HA and BMP7}

We further tested if the G7 moiety of the bivalent VHH was still able to bind to BMP7 after being immobilized on HA plates. For this purpose, pure HA plates were incubated with serial 
concentrations of VHHs (ranging from 0 to $7 \mu \mathrm{M}$ ) in 1\% BSA in PBS for two hours at RT after blocking them with 2\% BSA for two hours and with 4\% Marvel (Marvel original dried skimmed milk) subsequently for one hour. Subsequently, the HA plates were incubated with hBMP7 (300 ng/ml) and mouse anti-hBMP7 $(1 \mu \mathrm{g} / \mathrm{ml})$ antibody for one hour at RT. In between these steps, the wells were washed with PBST and PBS. Bound anti-hBMP7 was detected by incubation with a donkey anti-mouse antibody coupled to HRP in 1\% BSA in PBS (1 hour, RT). The amount of HRP was quantified by addition of OPD in the presence of $\mathrm{H}_{2} \mathrm{O}_{2}(30 \mathrm{~min}$, RT). The reaction was stopped by addition of $\mathrm{H}_{2} \mathrm{SO}_{4}$. Subsequent measurements were performed at $490 \mathrm{~nm}$.

\section{Biological activity of VHH G7 and bihead G7-MA10}

\section{1) Alkaline phosphatase (ALP) assay}

The $\mathrm{C} 2 \mathrm{C} 12$ cell line was used for osteogenic differentiation. Cells were cultured in DMEM (Gibco) supplemented with $10 \%$ fetal bovine serum (FBS, Cambrex), $100 \mathrm{U} / \mathrm{ml}$ penicillin (Gibco) and $100 \mu \mathrm{g} / \mathrm{ml}$ streptomycin (Gibco), and were incubated at $37^{\circ} \mathrm{C}$ in humidified atmosphere and 5\% $\mathrm{CO} 2$. To perform differentiation assays, cells were seeded at a density of 10,000 cells $/ \mathrm{cm}^{2}$ (day 0). Upon reaching confluence (day 4) cells were cultured for 3 days with ascorbic acid (50 $\mu \mathrm{g} / \mathrm{ml}$; Sigma Aldrich) and stimulated with BMP7 (300 ng/ml; R\&D Systems) in the presence or absence of VHH G7 $(1 \mu \mathrm{g} / \mathrm{ml})$ or G7GS10MA10. At day 7, cells were washed with PBS and lysed with CDPStar lysis buffer (Roche). To evaluate ALP activity, cell lysate was added to CDPStar reagent (Roche) and luminescence was measured using Vector Microplate Luminometer (Promega). The luminescence units were corrected for DNA content. DNA concentration was determined via proliferation assay according to the manufacturer's protocol (CyQuant Cell Proliferation Assay Kit, Invitrogen).

\section{2) ALP staining and imaging}

Pure HA plates were placed in a 96-well plate (flat bottom) and sterilized with $70 \%$ ethanol for 
2 hours. $\mathrm{C} 2 \mathrm{C} 12$ culture medium was added to the wells for 2 hours. Bihead VHH (1 $\mu \mathrm{M})$ was added to the wells in 1\% BSA in PBS and kept at RT for 2 hours. hBMP7 (300 ng/ml) was added to the wells in PBS and left for 2 hours. In between these steps, the wells were washed with PBS. C2C12 cells, at a seeding density of 10,000 cells/well, were added to the wells together with ascorbic acid. After three days, the ALP expression on HA plates was determined by the Sigma Alkaline phosphatase kit following the protocol. The image was taken by stereoscopic microscope after counter-staining the cell nuclei with nuclear fast red.

\section{VHHs in vivo experiments}

\section{1) Labeling of MA10 and bihead G7GS10MA10 with near infrared IRDye $800 \mathrm{CW}$}

(IR)

On the basis of the promising results of VHH MA10 and bihead G7GS10MA10 binding to mineralized bone matrix, near infrared IRDye $800 \mathrm{CW}$ was chosen to label the VHHs for further detection in the vivo mouse model. VHH J3 was used as a non-HA binding control. A direct labeling strategy, consisting of site-directed labeling of the VHH's C-terminus, was used to avoid interference with antigen-antibody interaction. This was achieved through the genetic introduction of an unpaired cysteine at the VHH's C-terminus (MA10-CYS or G7GS10MA10CYS ), which could be conjugated to a maleimide containing IRDye $800 \mathrm{CW}$. VHH MA10CYS and G7GS10MA10-CYS were modeled using the online program I-TASSER for protein modeling [31-33]. The obtained VHH MA10-IR and G7GS10MA10-CYS-IR were tested for binding to HA or to HA and BMP7 simultaneously to confirm that the genetic modification and labeling did not interfere with the function of the VHHs.

\section{2) In vivo performance of MA10}

To assess the possibility of MA10 targeting mineralized bone, MA10-IR was intravenously injected in two groups of balb $\mathrm{C}$ nu/nu mice (Charles River, France). One group was injected with VHH MA10-IR (70 $\mu \mathrm{g} / 100 \mu \mathrm{l})$ and the other was injected with the negative control VHH 
J3-IR $(70 \mu \mathrm{g} / 100 \mu \mathrm{l})$. The PEARL Impulse imaging camera (Li-Cor. Lincoln, Nebraska) was used to image the mice. The mice were anesthetized with $2 \%$ Isoflurane and imaged in ventral and dorsal positions at $0,1,3,24,48$ and 72 hours and 7, 16 and 20 days post injection. The results were analyzed with Pearl Impulse software 3.01. The mice were sacrificed with $\mathrm{CO}_{2}$ at 20 days post injection. The organs and skeletons were isolated and imaged.

\section{3) In vivo performance of G7GS10MA10}

This study includes four experimental groups. Six animals from each group were used to measure the dual activities of bihead bound to the bone and BMP7 simultaneously, thus BMP7 was directed to the bone area by bihead targeting hydroxyapatite. Mice from group 1 were injected with IR-labeled bihead (20ug/100ul), mice from group 2 were injected with BMP7 only (15ug/100ul), mice from group 3 were injected with a mixture of IR-labeled bihead and BMP7 (20ug bihead and 15ug BMP7/100ul); the mixture was prepared 2 hours before injection. Group 4 was injected with PBS negative control. At day 20 (24 hrs before sacrifice), 3 mice of each group received another injection of BMP7 (15ug/100ul). IR imaging was performed at regular time intervals: at day $0,2 \mathrm{hrs}, 24 \mathrm{hrs}, 72 \mathrm{hrs}, 7 \mathrm{~d}, 14 \mathrm{~d}, 21 \mathrm{~d}$ in ventral and dorsal position. All groups were sacrificed 21 days after injection, and a number of organs and skeletons were collected and imaged.

\section{4) Microscopic images of the bone of IR-bihead injected mice}

In order to detect the fluorescence signal location inside bone, microscopic imaging was performed after methyl methacrylate (MMA) embedding. To make sample slides, skeleton samples were fixed in $10 \%$ formalin 24 hours after sacrifice. Samples were rinsed with PBS and put into a Tissue Processor to dehydrate at $70 \%$ ethanol for 4 hours, $80 \%$ ethanol for 4 hours, $90 \%$ ethanol for 4 hours, $96 \%$ ethanol for 4 hours, $100 \%$ ethanol for 4 hours and $100 \%$ ethanol for 4 hours. The dehydrated skeletons were incubated with MMA solution (135 ml Kplast A, $15 \mathrm{~mL} \mathrm{~K}$-plast B, $1.5 \mathrm{~g}$ initiator) for 1 week at $4{ }^{\circ} \mathrm{C}$; the solution was changed every two days. Once the MMA had thoroughly infiltrated the skeleton, MMA embedding was 
performed by incubation with MMA solution overnight at $4{ }^{\circ} \mathrm{C}$, followed by a water bath at $37^{\circ} \mathrm{C}$ until the MMA had hardened. A $300 \mu \mathrm{m}$ section was sliced from the MMA embedded skeleton with a diamond blade (LEILA SP1600) and imaged using an Odyssey Near Infrared imaging system coupled to a microscope.

\section{5) Immunochemistry for skeletons of IR-bihead injected mice}

In order to detect whether the injected BMP7 had been directed to the bone tissue by the bihead and following triggered BMP7 signal transduction, rabbit source anti-VHH (QVQ), anti-BMP7 (Peprotech) and anti-SMAD 1 (Santa Cruz) antibodies were used for the purpose of immunochemistry. After decalcification for 3 weeks in EDTA solution (solution changed every week), the injected mice skeleton was embedded in paraffin and a $5 \mu \mathrm{m}$ section was sliced. The slides were de-paraffinized in xylene and rehydrated with graded ethanol. The antigen was retrieved by a citrate buffer $(10 \mathrm{mM}$ pH6.0) $2 \mathrm{~min}$ in a microwave, then cooled down to RT. This was followed by blocking the endogenous-peroxidase by a 3\% H2O2 solution for 15 min at RT. After blocking in 5\% BSA in PBS for 1 hour, samples were incubated overnight with primary specific antibody diluted in blocking buffer (anti-VHH 10ug/ml, anti-BMP7 10ng/ml, anti-SMAD1 $10 \mathrm{ug} / \mathrm{ml}$ ) and $\mathrm{IgG}$ control antibody at $4{ }^{\circ} \mathrm{C}$. Followed by adding biotinylated goat anti-rabbit IgG (Abcam) for $30 \mathrm{~min}$, and HRP-strep in PBS $(2 \mathrm{ug} / \mathrm{ml})$ for $30 \mathrm{~min}$. Samples were incubated in DAB solution (Abcam) for $10 \mathrm{~min}$, hematoxylin was used to counterstain the nuclei for $10 \mathrm{sec}$. The samples were washed 3 times by PBS between each step. Nanozoomer was used to take an image.

\section{Statistical Analysis}

Statistical analyses were performed using GraphPad Prism version 5.00 for Windows, GraphPad Software (San Diego, California). The analyses were based on one-way ANOVA and a Tukey's Post-hoc test $(\mathrm{p}<0.05)$ among all samples or between samples and controls. Error bars indicated standard deviation. 


\section{Results}

\section{VHH targeting BMP7 (VHH-G7) production and characterization}

VHH was selected from phage display libraries constructed from RNA isolated from PBLs of llamas immunized with recombinant human BMP7 [37]. After two selection rounds, a clear enrichment was found on the antigen-coated wells in comparison with empty wells. Several clones were selected for further screening using binding to BMP7. From two master plates of $190 \mathrm{VHH}$ clones, the selection of individual clones was sized down by grouping on the basis of restriction patterns and an ELISA binding assay. One clone named VHH G7 (G7) was selected for further characterization based on binding specificity. The gene sequence was subcloned into expression plasmids and produced and purified as indicated in materials and methods. The sequence of clone G7 is shown in Figure 3A. The purity of the recombinantly produced VHH was assessed by SDS-PAGE and Coomassie blue staining (Figure 3B). A clear band was observed with a size of $16 \mathrm{kDa}$. To confirm the specific binding of VHH G7 to hBMP7, an ELISA was performed (Figure 3C). The data indicated that VHH G7 binds to hBMP7 with a $\mathrm{K}_{\mathrm{d}}$ of 2,2nM.

A

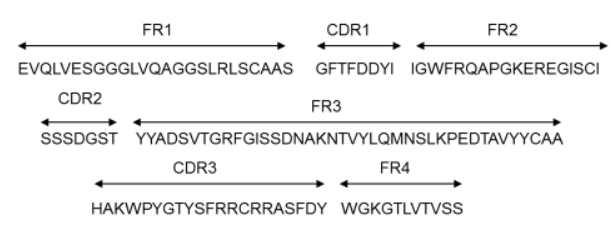

B

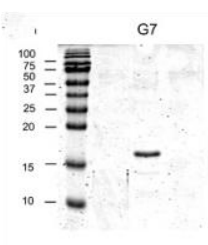

C

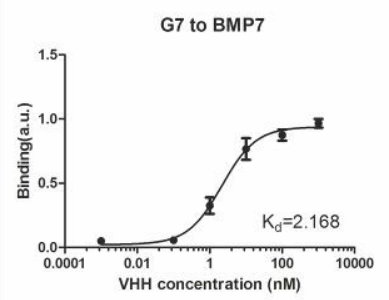

Figure 3. Characterization of VHHs targeting hBMP7. (A) The amino acid sequence of VHH-G7 targeting hBMP7. Frame works (FR) and the complementary determining regions (CDR) are indicated according to Chothia [39]. (B) Purified G7 was sized-separated by a 15\% SDS-PAGE and stained with Coomassie Brilliant Blue. Molecular weight markers (in kDa) are indicated at the left. (C) The specific binding of VHH-G7 to hBMP7 in ELISA. Data is expressed as the mean +/- standard deviation of at 
least 3 replicates.

\section{VHH targeting HA ( VHH MA10 ) production and characterization}

VHH were selected from a non-immunized phage library. The selections were performed on HA plates placed in wells of a 96-wells microtiter plate (Figure 4A). A total of two selection rounds were performed on HA plates to achieve an enrichment of phages binding to HA plates. Six single clones that showed binding to HA were selected during screening. After VHH expression and sequence determination, clone MA10 was selected for further characterization. VHH MA10 was purified from the periplasmic fraction of E.coli TG1 using TALON. The size and purity of the VHH were assessed by SDS-PAGE where a single band was detected of 16 $\mathrm{kDa}$. The specific binding of VHH MA10 to HA was tested by ELISA (Figure 4B). VHH MA10 showed binding to HA in a dose dependent manner with an apparent affinity of $\mathrm{K}_{\mathrm{d}}=$ $73.9 \mathrm{nM}$.

We next tested whether MA10 could be used for visualizing mineralized bone using explanted fetal mouse metatarsal. For this, VHH MA10 was labeled with Alexa fluor 647. An unrelated VHH was used as a negative control VHH 1B5 and was also labelled. The labeling efficiency obtained was 30\% and 50\% for VHH MA10-647 and 1B5-647, respectively. Next metatarsals were incubated with both VHHs. Fluorescent microscopy on the intact metatarsals revealed that VHH MA10-647 was specifically bound to the mineralized cartilaginous matrix of the primary center of ossification. In addition, binding to the developing bone collar in which mineralized bone matrix is deposited was observed (Figure 4C). In contrast, VHH 1B5-647 binding to mineralized extracellular matrix was strongly reduced, with the exception of some nonspecific fluorescence at the boundary of the mineralized and hypertrophic cartilage, and with faint fluorescence in the bone collar. Interestingly, Alexa Fluor 647 did not display any significant auto fluorescence using an emission filter of $692 \pm 40 \mathrm{~nm}$. 


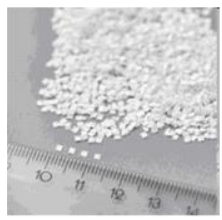

$\mathrm{B}$

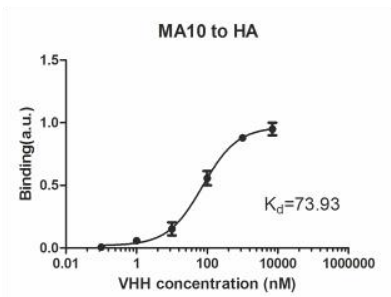

C

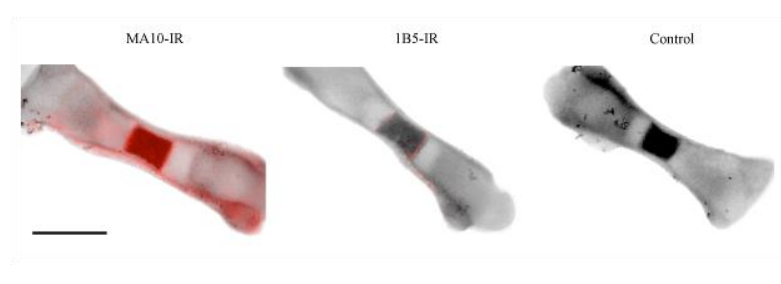

Figure 4. Characterization of VHHs targeting HA. (A) Photography of HA plates measuring approximately $1.5 \times 1.5 \times 0.1 \mathrm{~mm}$. (B) The specific binding of VHH-MA10 to HA by ELISA. Data is expressed as the mean +/- standard deviation of at least 3 replicates. (C) VHH-MA10 targeting mineralized extracellular matrix in mouse fetal metatarsals. Scale bar represents $0.25 \mathrm{~mm}$. N=3.

\section{Bihead G7-MA10 production and characterization}

In order to explore the potential of $\mathrm{VHH}$ with dual specificity in functionalizing biomaterial surfaces, a bispecific VHH consisting of an anti-BMP7 and an anti-HA was constructed (Figure 1D). PCR was used to link the genes of the two VHHs (Figure 5A) and to introduce a linker sequence between them, as described in material and methods (Figure 5B). The resulting bihead VHH is schematically illustrated in Figure 5C. Its structure was predicted by ITASSER demonstrating that the genetic fusion did not impact the exposure of the complementary determining regions (CDR) involved in antigen binding [17-19]. The fusion gene of the G7GS10MA10 was subcloned into expression plasmids and produced and purified as indicated in materials and methods. As shown in Figure 5D, the size of the produced bihead VHH had the expected molecular weight of $30 \mathrm{kDa}$, about twice that of the monovalent VHHs which have a weight of $16 \mathrm{kDa}$, as shown by SDS-PAGE.

In order to show whether the combination of MA10 and G7 in a bispecific VHH influences the binding of the MA10 part of the bihead VHH to HA and accordingly the G7 part to BMP7, the binding specificity was determined using ELISA. As shown in Figure 6, bihead VHHs show specific binding to HA with an apparent affinity of $\mathrm{K}_{\mathrm{d}}$ of $62.59 \mathrm{nM}$, and to hBMP7 with an 
apparent affinity of $\mathrm{K}_{\mathrm{d}}$ of $0.4 \mathrm{nM}$, respectively Figure 6A and B) which is in agreement with

A
v日月
67
Nh10
var
67
Ma10

B

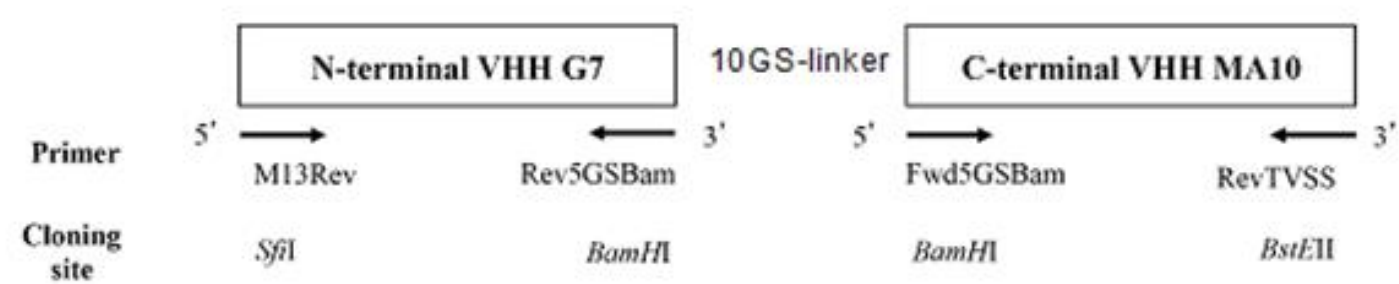

C

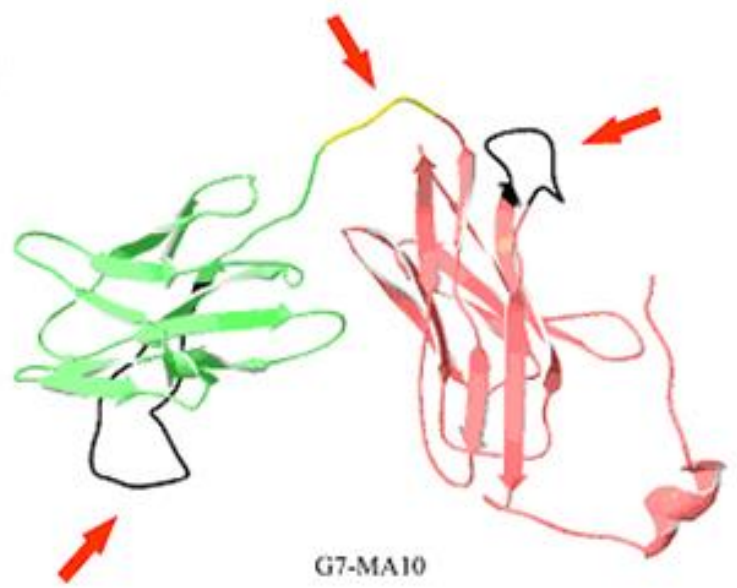

Bivalent VHH

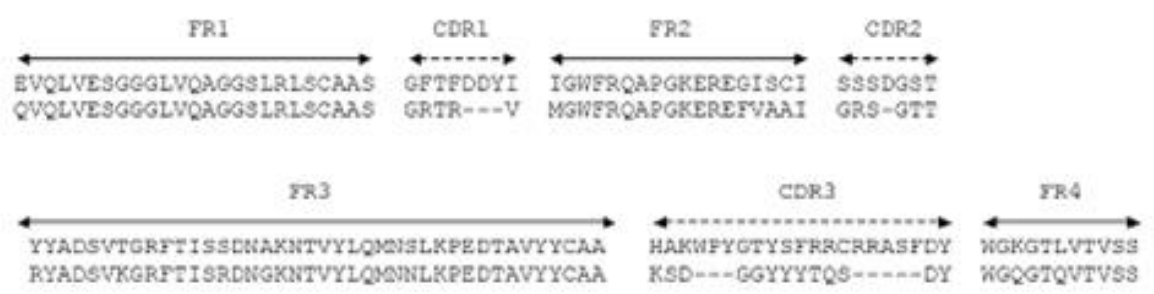


MA10 and G7-MA10 were sized-separated by a 15\% SDS-PAGE and stained with Coomassie Brilliant Blue. Molecular weight markers (in $\mathrm{kDa}$ ) are indicated at the left. Calculated molecular weight of the monovalent and bispecific VHHs are indicated at the right. (E) Binding specificity of bihead VHHs to HA by ELISA. Data is expressed as the mean +/- standard deviation of at least 3 replicates.

the affinities of the individual components for HA and hBMP7. In order to show if the bihead VHHs are also able to bind to HA and hBMP7 simultaneously, a binding curve was obtained for hBMP7 to the bihead which was first immobilized on a HA disc. As shown, the bihead was able of binding both HA and hBMP7 albeit that the binding to HA reduced the affinity of G7 for hBMP7.

A

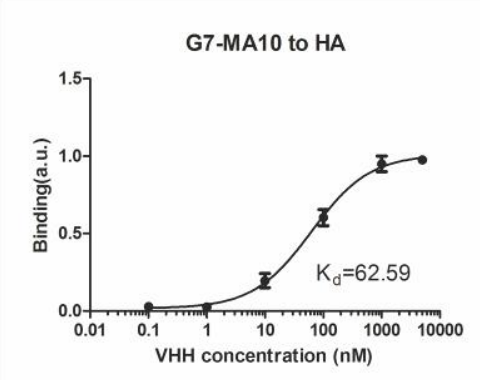

D

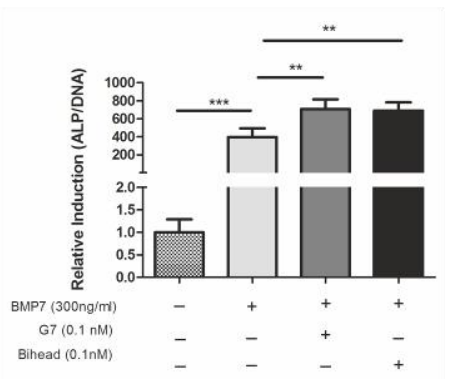

B

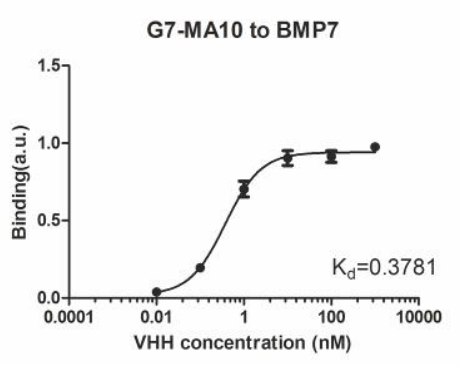

C

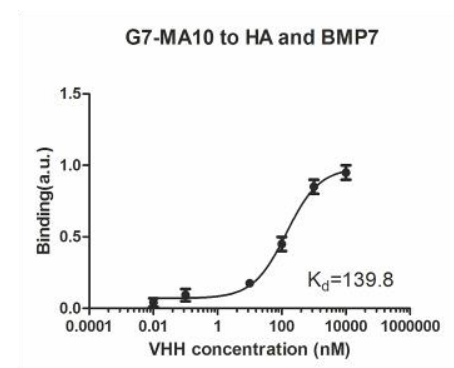

E

HA

HA+BMP7

Bihead+BMP7

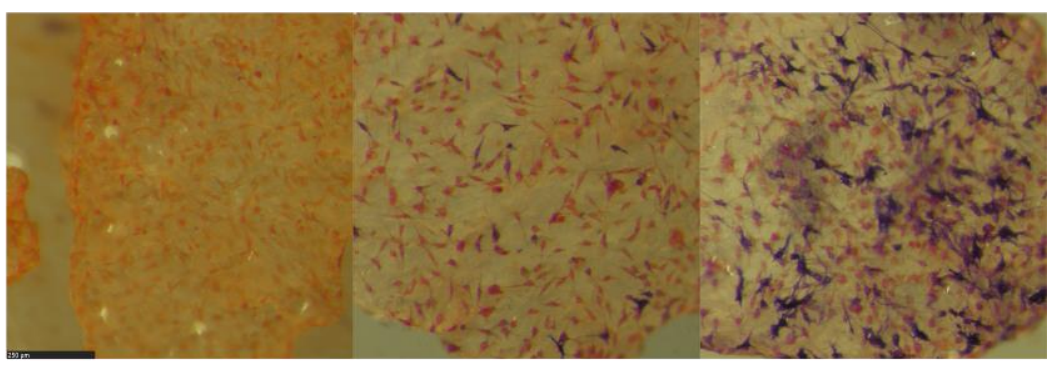

Figure 6. Binding specificity of bihead G7-MA10. (A) Binding specificity of bihead VHHs to HA and to (B) BMP7 by ELISA. (C) Dual binding specificity of bihead to HA and BMP7 by ELISA. BMP7 bind to bihead which was first immobilized on HA disc. Anti-BMP7 antibody was used to quantify the bound BMP7 as materials and methods discribed. Data is expressed as the mean $+/$ - standard deviation 
of at least 3 replicates. (D) Biological activity of G7 and bivalent G7-MA10 in C2C12 cells. The ALP activity was normalized to the total DNA, and expressed as relative induction with respect to control (non-treated cells). Data is expressed as mean $+/$ - standard deviations (error bars), $\mathrm{N}=6$. ** indicates significance of $\mathrm{p}<0.01 ; * * *$ indicates significance of $\mathrm{p}<0.001$. (E) HA discs were pre-incubated with bihead or BMP7 or bihead and BMP7 prior to seeding of C2C12 cells. After 3 days of culture ALP expression on HA plates was stained into blue by Sigma Alkaline phosphatize kit, the cell nuclei were counter-stained in red by nuclear fast red. Scale bar equals $250 \mu \mathrm{m}$.

The biological activity of VHH-G7 or bihead G7-MA10 hBMP7 was tested in C2C12 cells. ALP is an early marker of osteogenic differentiation, and it is strongly induced by hBMP7. Here we determined the effect of VHH-G7 or bivalent G7-MA10 in the presence or absence of hBMP7 in culture medium on Alkaline Phosphatase activity (ALP) over a 7-day period. As shown in Figure 6D, ALP activity was induced around 400-fold in the presence of hBMP7. Interestingly, when cells were co-treated with hBMP7 in the presence of VHH-G7 or bihead G7-MA10, a clearly superior induction of ALP activity was observed: Both VHHs increased ALP activity induced by BMP7 with 177\%. There was no significant difference between G7 and G7-MA10.

We next tested whether the potentiating effect of the bihead G7-MA10 on hBMP7 activity was preserved when the bihead was bound to HA. For this, C2C12 cells were cultured on pure HA plates after pre-incubation with bihead and/or hBMP7. The HA plates were extensively washed with PBS between steps before cells were seeded on top. Compared to control, pretreatment of HA discs with hBMP7 marginally induced ALP acitivity. Much stronger induction of ALP activity was found in discs pretreated with both the bihead and BMP7. This indicated that the bihead was able to sequester hBMP7 out of the medium on the biomaterial surface and that this local enrichment is sufficient to induce ALP activity in the C2C12 cells (Figure 6E). 


\section{Infra-red labeled VHH MA10 binds to mineralized skeleton}

In order to evaluate the potential of MA10 as a probe for optical imaging of bone tissue, MA10 was labeled using a near infrared label yielding MA10-IR. The label process did not affect the binding specificity of VHH to HA (Supplemental Figure 1A), determined by ELISA. Next, nude mice were intravenously injected with MA10-IR in the tail vein and imaged at several time points post-injection (Figure 7A). Littermates were treated in the same way with the non-

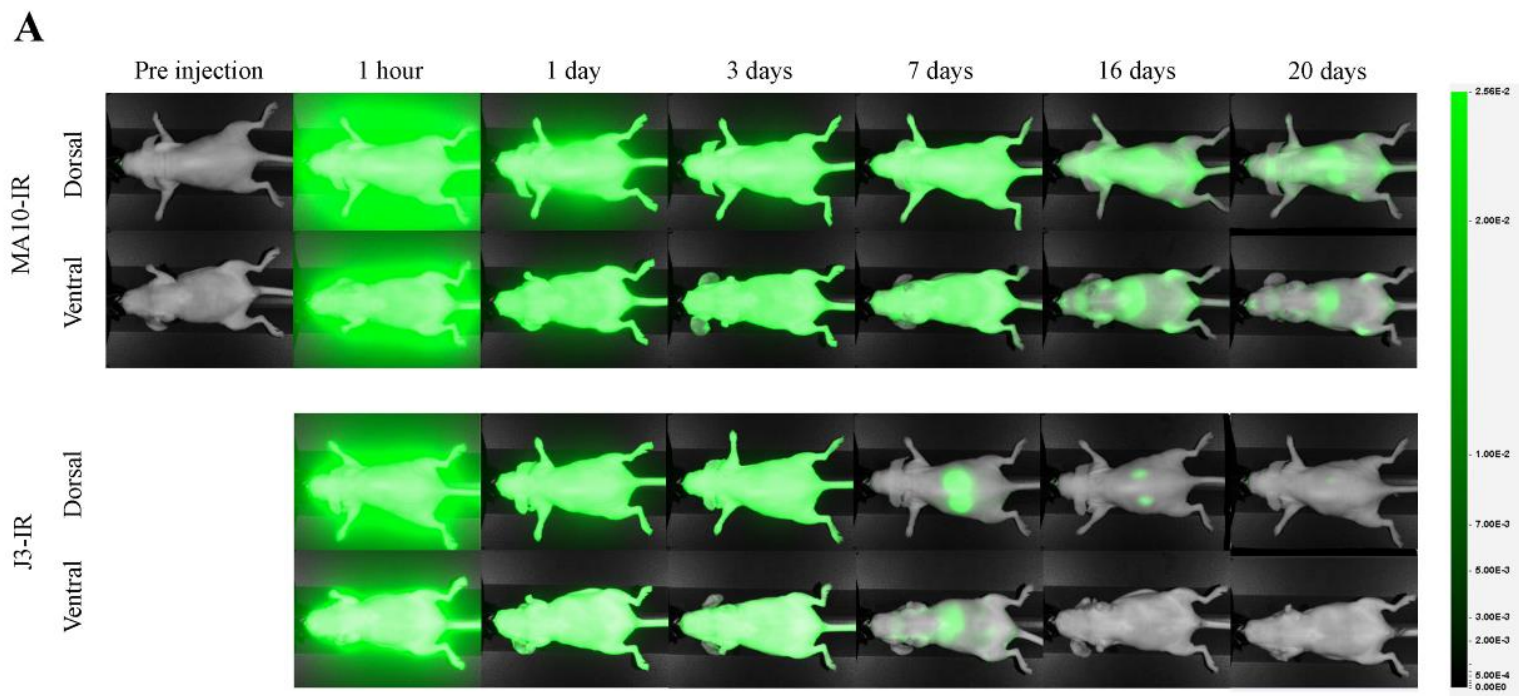

B
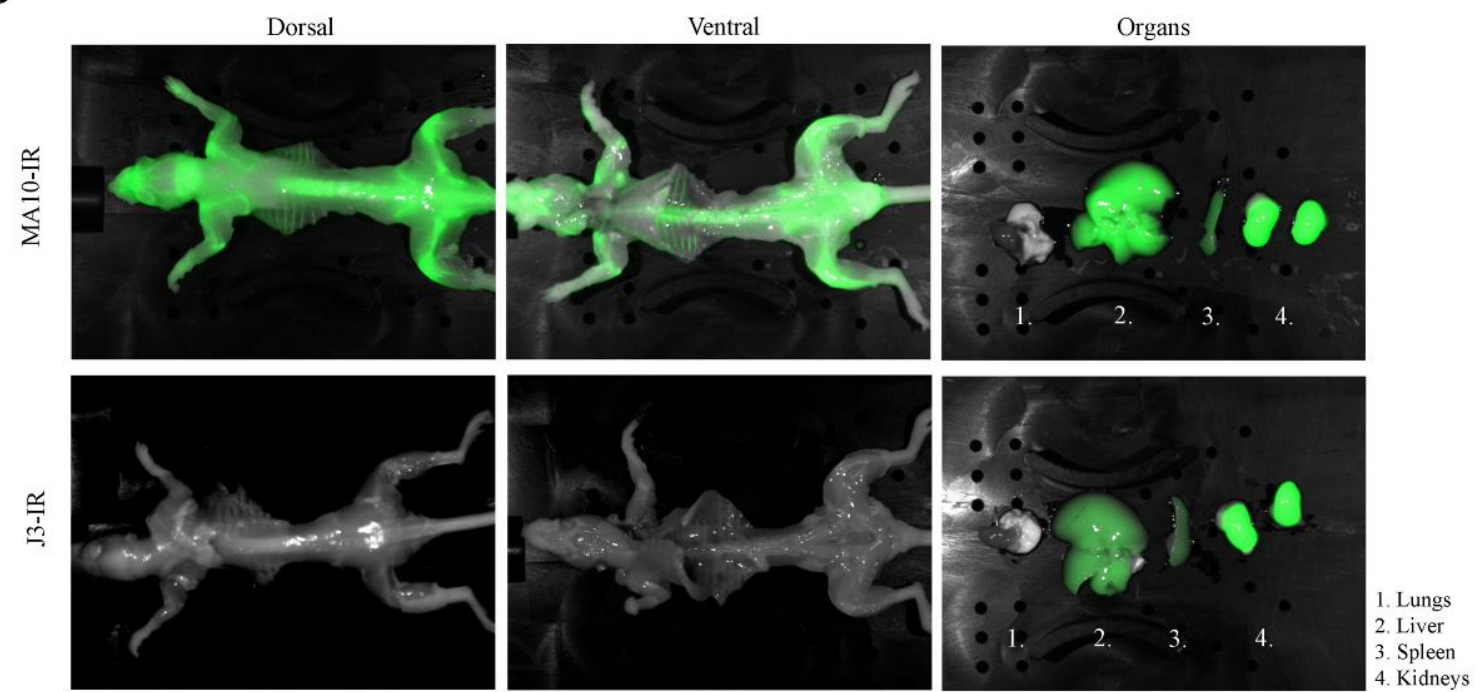

Figure 7. Fluorescence images over time of VHH MA10-IR (70 $\mu \mathrm{g} / 100 \mu \mathrm{l}$ injection sample) and negative control J3-IR (70 $\mu \mathrm{g} / 100 \mu$ linjection sample) upon intravenous injection in Balb C nu/nu 
mice. (A) Live imaging. Dorsal and ventral images of the same mouse were obtained throughout a time period of 20 days after injection. A representative image of an $n=2$ experiment is shown. (B) Postmortem imaging. At day 20, after the mice were sacrificed, the skeleton and organs were isolated and imaged. The VHHs were injected intravenously in the tail vein.

binding VHH J3-IR as negative control, and images of the two groups were compared. Images taken at early time points showed IR fluorescence throughout the whole body of the mice in the case of MA10-IR (up to 7 days post-injection) and in the case of J3-IR (up to 3 days postinjection). At day 20 fluorescent label was still detectable in the skeleton of mice injected with MA10-IR, in contrast to J3-IR injected mice. After sacrificing, the skeleton and organs were isolated and imaged (Figure 7B). The dorsal and ventral images showed a clearly defined skeleton with high fluorescence measured for MA10-IR. Specific accumulation of MA10-IR occurred in mineralized bones. In contrast, no fluorescence was measured for the VHH J3-IR in the skeleton. For both VHH, liver, spleen and kidneys show fluorescence after 20 days. Fluorescence was clearly visible at the kidneys for both groups. Obviously, J3-IR was cleared from the body much faster than MA10-IR, suggesting that targeting the skeleton through binding to hydroxyapatite extended the retention time of the $\mathrm{VHH}$ in the body considerably to at least 20 days.

\section{Bihead VHHs efficiently direct hBMP7 to bone by targeting hydroxyapatite}

In order to evaluate whether hBMP7 can be directed to the bone area by the dual binding activity of bihead G7-MA10 to BMP7 and HA simultaneously, bihead G7-MA10 was labeled by IR to G7-MA10-IR. ELISA result showed the labeling did not affect the binding specificity of the bihead to HA (Supplemental Figure 1B). Next, the mix of hBMP7 and IR labeled bihead was injected into mice. hBMP7 only or labeled bihead only was injected as control. At day 20 (24 hrs before sacrifice), another injection of BMP7 was performed. As Figure 8A shows, at early time points IR fluorescence was present in the whole body; two weeks later fluorescent signal was difficult to measure in the living mice. However, after sacrifice 3 weeks later, fluorescence was still detectable in the skeleton or organs like liver, spleen and kidneys of 
group 1 and group 3 mice, which contained labeled bihead. This means the specific accumulation of bihead-IR had occurred at mineralized bones. It is reasonable that the signal of labeled bihead injection mice is weaker compared to labeled MA10 injection mice, since the dose was 4 times lower than that of VHH-MA10-IR. In order to further detect the fluorescent signal location inside bones, microscopic imaging was performed. Most fluorescent signals come from the trabecula (Figure 8D), which strongly indicated that the bihead was targeting sites of active bone remodeling.

A

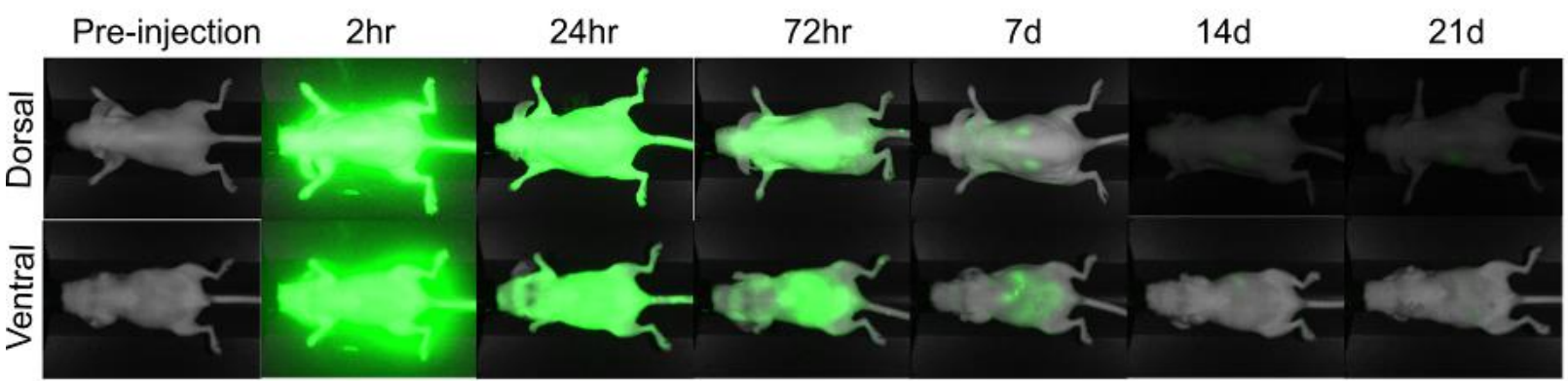

B

Dorsal

Ventral

Organs

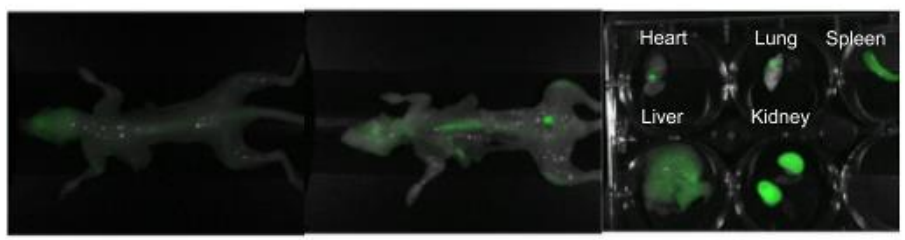

C

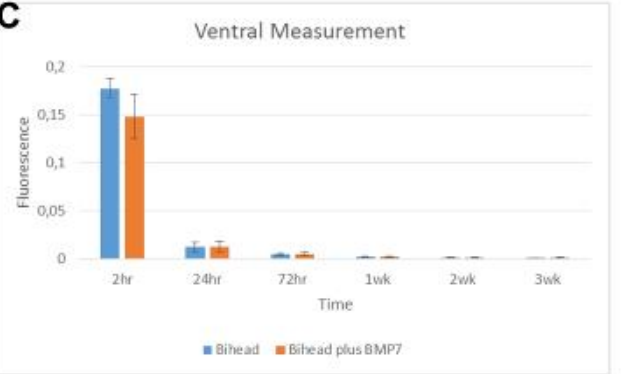

D

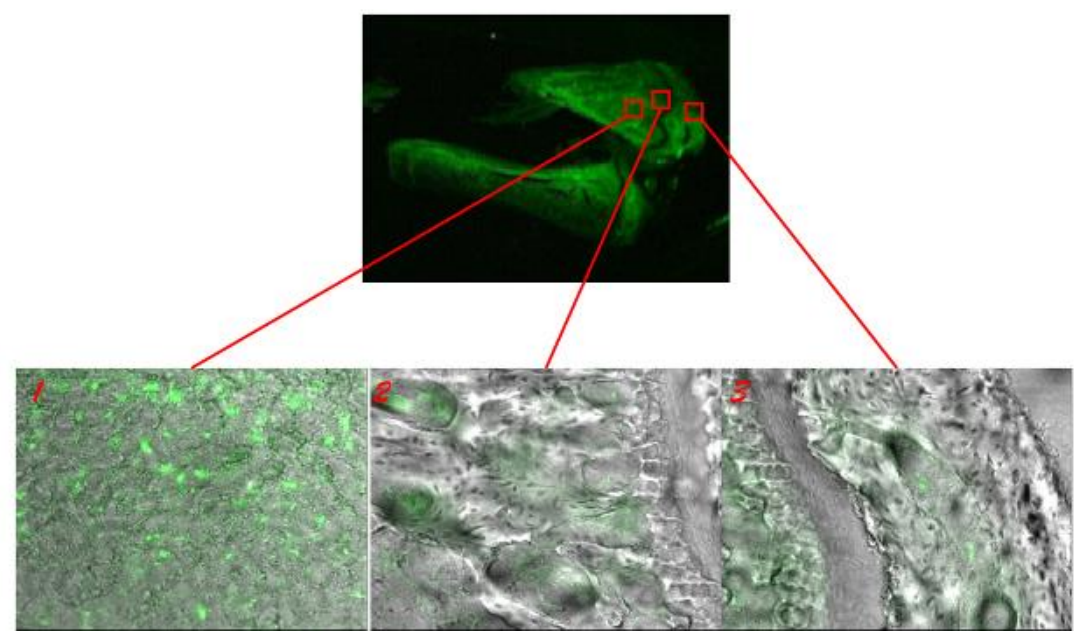

Figure 8. Fluorescence images over time of VHH G7-MA10-IR $(20 \mu \mathrm{g} / 100 \mu \mathrm{l}$ injection sample) 
upon intravenous injection in Balb C nu/nu mice. (A) Live imaging. Dorsal and ventral images of the same mouse of group 1 or group 3 were obtained throughout a time period of 3 weeks after injection. A representative image is shown of an $n=6$ experiment. (B) Postmortem imaging. At day 20, after the mice were sacrificed, the skeleton and organs were isolated and imaged. (C) Quantification of fluorescence signals of group 1 or 3. (D) Microscopic imaging of bihead-IR injected mice tibia. 1, trabecula area; 2 growth plate area; 3 articular cartilage and subchondrondral bone plate.

A

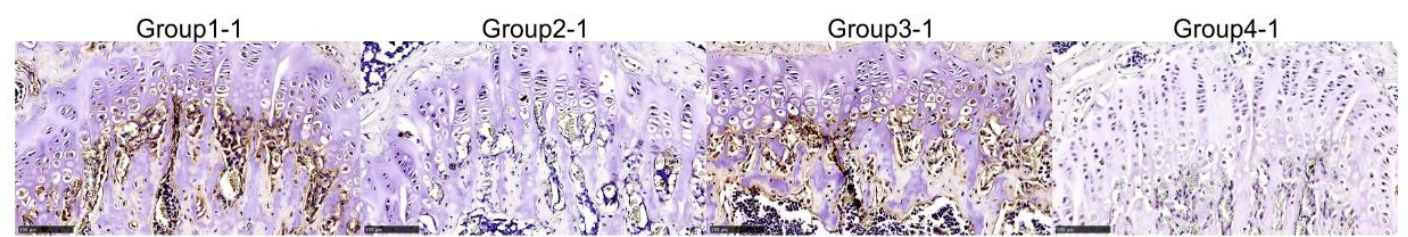

Anti-VHH

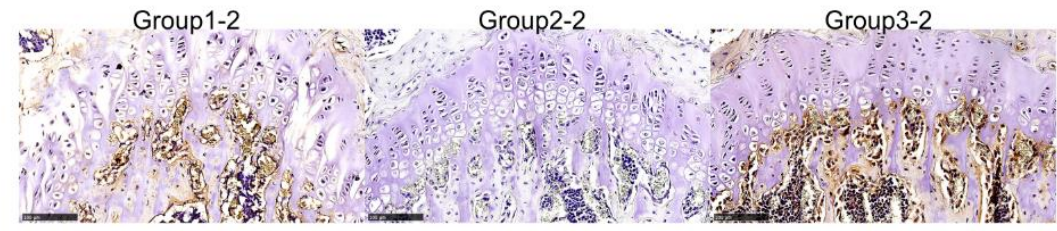

B

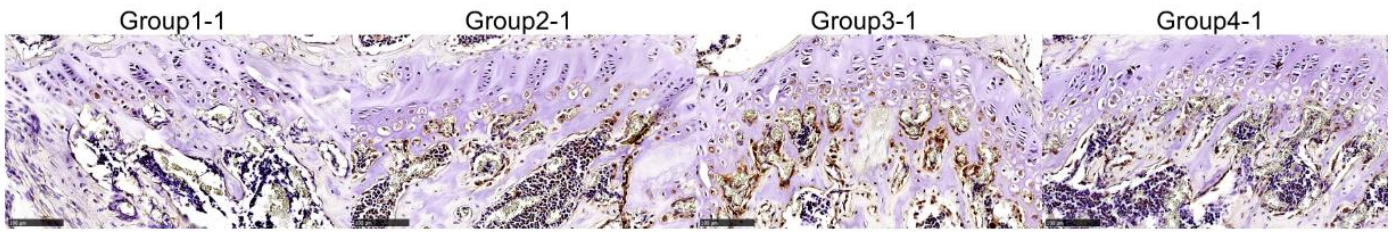

Anti-BMP7

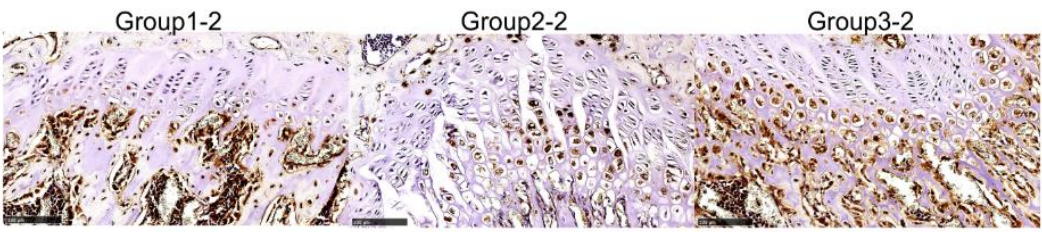

C

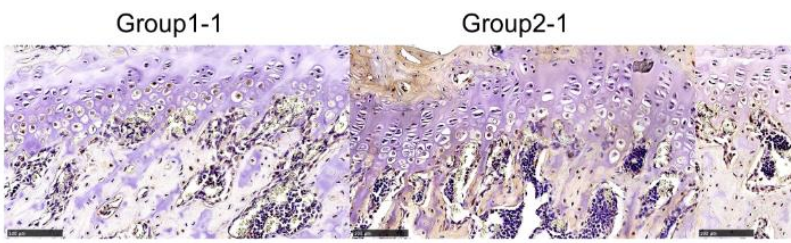

Anti-SMAD1

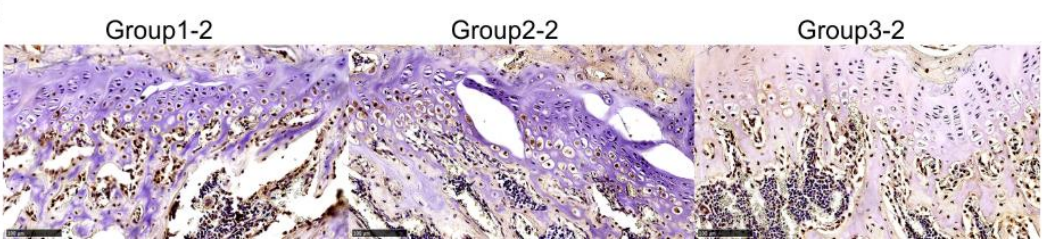

Figure 9. Immunohistochemistry for VHH, hBMP7 and SMAD1 in VHH G7-MA10-IR injectied 
mice. (A) Anti-VHH, group 1-1 indicates mice injected with G7-MA10-IR only; group 2-1 indicates mice injected with BMP7 only; group 3-1 indicates mice co-injected with G7-MA10-IR and BMP7; group 1-2 indicates mice injected with G7-MA10-IR plus $24 \mathrm{hrs}$ prior sacrifice with an additional hBMP7 injection before sacrifice; group 2-2 indicates mice injected with hBMP7 3 weeks and hBMP7 24hr before injection; group 3-2 indicates mice injected by the mix of G7-MA10-IR and BMP7 plus a second injection of hBMP7 $24 \mathrm{hrs}$ before sacrifice; group 4 is the negative control group injected with placebo only. (B) Anti-BMP7; (C) Anti-SMAD1.

Immunohistochemistry confirmed the presence of VHH in bone. Strong staining was observed in the mineralized cartilage of the growth plate and in trabeculae in the bihead injected groups (Figure 9A). The non-bihead injected groups did not show staining. Immunohistochemical analysis of BMP7 after 21 days showed clear enrichment of hBMP7 in the experimental group that received a co-injection of the bihead with hBMP7 (Figure 9B). Interestingly, in mice that received a second injection with hBMP7 1 day before sacrifice, hBMP7 staining was increased compared to the non-injected animals. Remarkably, the second injection resulted in a very marked enrichment of hBMP7 in animals that had received an injection with the bihead 20 days earlier. The staining for hBMP7 co-localized with the immunohistochemical staining for the VHHs. This suggested that the hBMP7 binding part of the bifunctional VHH was still capable of sequestering hBMP7 out of circulation even 3 weeks after injection. Immunohistochemical staining of SMAD1 showed a similar picture as for hBMP7 (Figure 9C). Only the group which received a co-injection of bihead and hBMP7 showed increased staining. The staining co-localized with both VHH and hBMP7 staining. SMAD1 staining was also increased in the groups that received a second injection of hBMP7 1 day before sacrifice particularly in those mice that were 20 days earlier injected with the bihead (Figure 9C).

\section{Discussion}

In tissue engineering, much effort has been put into the development of bioactive scaffolds 
with controlled growth factor delivery in recent years. Growth factors can be used to biofunctionalize biomaterials, which have been put forth as a promising therapeutic strategy to stimulate tissue repair and potentially tissue regeneration. However, limitations to the immobilization of growth factors to biomaterials are present [5]. VHHs can be used for the modification of biomaterials to enhance their biological instructive properties through coupling to biomaterial surfaces. In this way, VHHs can act as concentrators of endogenous and/or exogenous growth factors, creating a depot function at the biomaterial cell surface for sustained release. Furthermore, bio-functional VHH antibodies are capable of simultaneously binding biomaterial or ECM and growth factors to enhance the tissue response during tissue regeneration. Unlike growth factors that are highly susceptible to manipulation, such as formulation of these factors in a drug depot or directly coupling to a biomaterial, this system does not require genetic or chemical modifications of growth factors, as no covalent coupling between growth factor and biomaterials is required [34, 35]. In addition, in tissue engineering it is of the utmost importance to design a scaffold/implant with spatiotemporal release of growth factors that matches the natural tissue regeneration pattern and remodeling phases [36]. VHH can be a versatile tool to accomplish spatiotemporally controlled release.

In this research, ECM binding VHHs that were selected against HA and a growth factor binding VHH that was selected against hBMP7 were studied. We first generated, isolated and characterized a VHH (VHH-G7) that was able to bind to hBMP7 via an immunized library of VHH, and a VHH (VHH-MA10) that was able to bind to HA which was selected from a nonimmunized library. Selection was done with phage display techniques, which allowed for efficient identification of these antibody fragments. Phage display is a well-known and established technology which has already been utilized for the selection of peptides binding to HA [37]. In this study, we have demonstrated for the first time the possibility of selecting VHH binding with high affinity to non-peptide epitopes present in HA, by means of phage display from a non-immunized library, which contains a myriad of VHH-phages with affinity towards a large variety of epitopes.

Bihead VHH combining G7 and MA10, along with 10 amino acid long glycine-serine linker 
molecules, was synthesized and evaluated for bone tissue engineering. We demonstrated a unique strategy in functionalizing biomaterials and modulating growth factors in a single step. The bihead VHHs could bind to the HA on one hand and to hBMP7 on the other specifically and in a dose dependent manner. We showed that the bivalent construction did not affect the binding to either epitope. Furthermore, in a cell biological assay, both the monovalent VHH G7 and the bivalent VHH (G7-MA10) potentiated the biological activity of hBMP7 in induction of ALP in the presence of hBMP7. Various possibilities can explain the potentiating effect of a VHH. For example, the conjugation of VHH G7 or bihead with hBMP7 may induce a conformational change in hBMP7, facilitating the binding to its receptor [38]. In addition, it is known that the activity of BMP7 is potently inhibited by the presence of antagonists such as Noggin, Chordin and Gremlin [39]. It is conceivable that VHH G7 or bihead interferes with this antagonistic activity by preventing their binding and thus counteracting this antagonistmediated inactivation of hBMP7 [40].

VHHs can also be used as imaging tools when associated with fluorophores or other imaging agents [29], and help clarify the mechanism behind the signal transduction of growth factor receptors [41]. In this study, mice were injected with near infrared fluorophore labeled VHH to assess the possibility of targeting mineralized bone. The small VHH size allowed for a rapid VHH distribution throughout the mouse body after one hour post-injection. IR fluorescence was visible throughout the body of the mouse at day 1, after which the IR fluorescence decreased, but still showed specific binding of MA10-IR to skeletal tissue. Binding to skeletal tissue resulted in long-term retention of the VHHs in the body. To our knowledge, this was the first time a VHH was used as a targeting molecule in bone tissue. This non-invasive technology, by means of labeled VHHs, can be employed for early detection of skeletal disorders and/or the identification and delineation of the diseases, which makes this a potential powerful tool with applications in bone tissue engineering, guided surgery, treatment of bone metastasis and treatment monitoring [42].

Labeled bihead G7-MA10-IR only or bihead mixed with hBMP7 was injected into nude mice to further evaluate drug targeting. The bihead injected mice demonstrated the specific binding 
of G7-MA10-IR to mouse skeletons and long-term retention of the VHHs in the body in line with clone MA10-IR. Subsequently, a cross-section of the mouse bones was performed in order to determine the diffusion possibility of the VHHs and the specific binding of the VHHs to bone. The microscopic images show that the signal comes mainly from the trabecula, which strongly indicates that bihead accumulate at sites with the most active bone remodeling. As we hypothesized previously, the IHC results confirmed that hBMP7 can gather in the bone area through binding to bihead, which then binds to bone. This is also the first time that bivalent $\mathrm{VHH}$ has been used to explore their potential use as a targeting vector of growth factors to areas of interest. The application of bivalent $\mathrm{VHH}$ can be further extended to other biomaterials, growth factors and phases of tissue development. If proper targeting VHHs for binding to biomaterials are available, simple injections (even post implantation of scaffold) of bivalent VHHs can be used to improve the bio-functionality of the scaffold by capturing/ sequestering the adequate growth factor. Therefore, VHHs could be used for drug targeting. The construct G7-MA10 which we designed in this study is a promising delivery vehicle for bone anabolic drugs that could be used for treating systemic bone disease, for example by coating the VHHs with liposomes, conjugating cytotoxic drugs for treatment of bone metastasis or by genetic engineering in which the $\mathrm{VHH}$ targeting bone is part of a multivalent antibody complex. The versatility of VHH allows for endless combinations.

The construction of bivalent and multivalent VHHs offers endless combinations of systems, where the binding moieties can be selected according to the application. Furthermore, VHH sequences can also be varied to obtain VHHs with tailored properties. VHHs can also be used as a core structure for the grafting of sequences known for binding elements or with different affinities such as peptides. In addition, the use of different linker sizes and cleavable linkers could be explored. Linkers with a specific amino acid sequence, which can be cleaved at different stages of inflammation or phases of tissue reconstruction, are of the utmost importance to achieve a complex release pattern of bioactive compounds. The versatile properties of VHHs render this novel antibody platform very valuable in research based on tissue engineering, where several disease mechanisms need both agonists and antagonists. Finally, polyvalent bioactive molecules offer the possibility of more effective therapeutics. 


\section{References}

1. Langer, R. and J.P. Vacanti, Tissue engineering. Science, 1993. 260(5110): p. 920-6.

2. Zhou, H. and J. Lee, Nanoscale hydroxyapatite particles for bone tissue engineering. Acta Biomaterialia, 2011. 7(7): p. 2769-2781.

3. Bessa, P.C., M. Casal, and R.L. Reis, Bone morphogenetic proteins in tissue engineering: The road from laboratory to clinic, part II (BMP delivery). Journal of Tissue Engineering and Regenerative Medicine, 2008. 2(2-3): p. 81-96.

4. Lee, E.J., F.K. Kasper, and A.G. Mikos, Biomaterials for tissue engineering. Annals of Biomedical Engineering, 2014. 42(2): p. 323-337.

5. Lee, K., E.A. Silva, and D.J. Mooney, Growth factor delivery-based tissue engineering: general approaches and a review of recent developments. J R Soc Interface, 2011. 8(55): p. $153-70$.

6. Koria, P., Delivery of growth factors for tissue regeneration and wound healing. BioDrugs, 2012. 26(3): p. 163-75.

7. Luginbuehl, V., et al., Localized delivery of growth factors for bone repair. European Journal of Pharmaceutics and Biopharmaceutics, 2004. 58(2): p. 197-208.

8. Tayalia, P. and D.J. Mooney, Controlled growth factor delivery for tissue engineering. Advanced Materials, 2009. 21(32-33): p. 3269-3285.

9. Ansari, S., et al., Immobilization of murine anti-BMP-2 monoclonal antibody on various biomaterials for bone tissue engineering. Biomed Res Int, 2014. 2014: p. 940860.

10. Liu, H., et al., Fc Engineering for Developing Therapeutic Bispecific Antibodies and Novel Scaffolds. Front Immunol, 2017. 8: p. 38.

11. Chames, P., et al., Therapeutic antibodies: successes, limitations and hopes for the future. Br J Pharmacol, 2009. 157(2): p. 220-33.

12. Deffar, K., et al., Nanobodies - The new concept in antibody engineering. African Journal of Biotechnology, 2009. 8(12): p. 2645-2652. 
13. Wesolowski, J., et al., Single domain antibodies: promising experimental and therapeutic tools in infection and immunity. Med Microbiol Immunol, 2009. 198(3): p. 157-74.

14. Muyldermans, S., Single domain camel antibodies: Current status. Reviews in Molecular Biotechnology, 2001. 74(4): p. 277-302.

15. Harmsen, M.M. and H.J. De Haard, Properties, production, and applications of camelid single-domain antibody fragments. Applied Microbiology and Biotechnology, 2007. 77(1): p. 13-22.

16. Arbabi Ghahroudi, M., et al., Selection and identification of single domain antibody fragments from camel heavy-chain antibodies. FEBS Letters, 1997. 414(3): p. 521-526.

17. Hamers-Casterman, C., et al., Naturally occurring antibodies devoid of light chains. Nature, 1993. 363(6428): p. 446-448.

18. Muyldermans, S., et al., Camelid immunoglobulins and nanobody technology. Veterinary Immunology and Immunopathology, 2009. 128(1-3): p. 178-183.

19. Wang, Y., et al., Nanobody-derived nanobiotechnology tool kits for diverse biomedical and biotechnology applications. Int J Nanomedicine, 2016. 11: p. 3287-303.

20. Hassanzadeh-Ghassabeh, G., et al., Nanobodies and their potential applications. Nanomedicine, 2013. 8(6): p. 1013-1026.

21. Heise, U., J.F. Osborn, and F. Duwe, Hydroxyapatite ceramic as a bone substitute. International Orthopaedics, 1990. 14(3): p. 329-338.

22. Vo, T.N., F.K. Kasper, and A.G. Mikos, Strategies for controlled delivery of growth factors and cells for bone regeneration. Adv Drug Deliv Rev, 2012. 64(12): p. 1292309.

23. Bessa, P.C., M. Casal, and R.L. Reis, Bone morphogenetic proteins in tissue engineering: The road from the laboratory to the clinic, part I (basic concepts). Journal of Tissue Engineering and Regenerative Medicine, 2008. 2(1): p. 1-13.

24. Kim, M. and S. Choe, BMPs and their clinical potentials. BMB Reports, 2011. 44(10): p. 619-634. 
25. Strokappe, N., et al., Llama antibody fragments recognizing various epitopes of the CD4bs neutralize a broad range of HIV-1 subtypes A, B and C. PLoS One, 2012. 7(3): p. e33298.

26. Frenken, L.G., et al., Isolation of antigen specific llama VHH antibody fragments and their high level secretion by Saccharomyces cerevisiae. J Biotechnol, 2000. 78(1): p. $11-21$.

27. Roovers, R.C., et al., Efficient inhibition of EGFR signaling and of tumour growth by antagonistic anti-EFGR Nanobodies. Cancer Immunol Immunother, 2007. 56(3): p. 303-317.

28. El Khattabi, M., et al., Llama single-chain antibody that blocks lipopolysaccharide binding and signaling: prospects for therapeutic applications. Clin Vaccine Immunol, 2006. 13(10): p. 1079-86.

29. Oliveira, S., et al., Targeting tumors with nanobodies for cancer imaging and therapy. J Control Release, 2013. 172(3): p. 607-17.

30. Haaijman, A., et al., Inhibition of terminal chondrocyte differentiation by bone morphogenetic protein $7(\mathrm{OP}-1)$ in vitro depends on the periarticular region but is independent of parathyroid hormone-related peptide. Bone, 1999. 25(4): p. 397-404.

31. Zhang, Y., I-TASSER server for protein 3D structure prediction. BMC Bioinformatics, 2008. 9: p. 40.

32. Roy, A., A. Kucukural, and Y. Zhang, I-TASSER: a unified platform for automated protein structure and function prediction. Nat Protoc, 2010. 5(4): p. 725-38.

33. Roy, A., J. Yang, and Y. Zhang, COFACTOR: an accurate comparative algorithm for structure-based protein function annotation. Nucleic Acids Res, 2012. 40(Web Server issue): $\mathrm{p} . \mathrm{W} 471-7$.

34. van der Linden, R.H., et al., Comparison of physical chemical properties of llama VHH antibody fragments and mouse monoclonal antibodies. Biochim Biophys Acta, 1999. 1431(1): p. 37-46.

35. Harmsen, M.M. and H.J. De Haard, Properties, production, and applications of camelid single-domain antibody fragments. Appl Microbiol Biotechnol, 2007. 77(1): p. $13-22$. 
36. Lowery, J.W. and M.P. de Caestecker, BMP signaling in vascular development and disease. Cytokine Growth Factor Rev, 2010. 21(4): p. 287-98.

37. Jin, H.E., W.J. Chung, and S.W. Lee, Phage display for the discovery of hydroxyapatite-associated peptides. Methods Enzymol, 2013. 532: p. 305-23.

38. Strange, P.G., Agonist binding, agonist affinity and agonist efficacy at G proteincoupled receptors. Br J Pharmacol, 2008. 153(7): p. 1353-63.

39. Bessa, P.C., M. Casal, and R.L. Reis, Bone morphogenetic proteins in tissue engineering: the road from the laboratory to the clinic, part I (basic concepts). J Tissue Eng Regen Med, 2008. 2(1): p. 1-13.

40. Groppe, J., et al., Structural basis of BMP signalling inhibition by the cystine knot protein Noggin. Nature, 2002. 420(6916): p. 636-42.

41. Heukers, R., et al., Endocytosis of EGFR requires its kinase activity and $N$-terminal transmembrane dimerization motif. J Cell Sci, 2013. 126(Pt 21): p. 4900-12.

42. de Boer, J., C. van Blitterswijk, and C. Lowik, Bioluminescent imaging: emerging technology for non-invasive imaging of bone tissue engineering. Biomaterials, 2006. 27(9): p. 1851-8. 
Supplementary Figure S1. The binding specificity of MA10-IR and bihead G7-MA10-IR to HA or HA and BMP7 by ELISA. (A) The binding specificity of MA10-IR to HA by ELISA. (B) The dual binding specificity of G7-MA10-IR to HA and BMP7 by ELISA. BMP7 bind to labeled bihead which was first immobilized on HA disc. Anti-BMP7 antibody was used to quantify the bound BMP7 as materials and methods discribed. Data is expressed as the mean $+/-$ standard deviation of at least 3 replicates, $\mathrm{N}=8$.

A

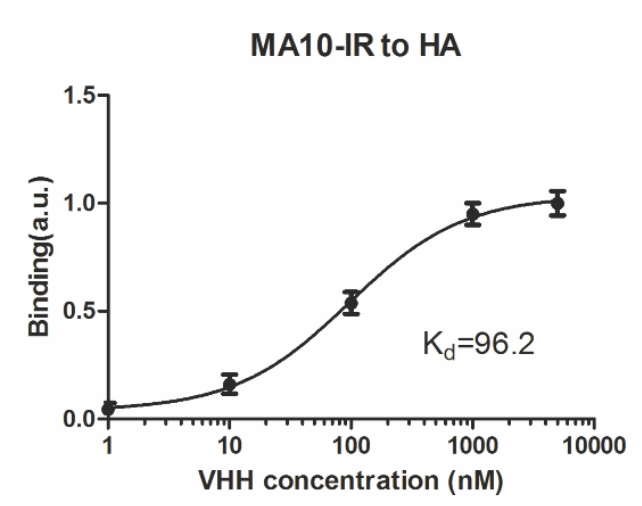

B

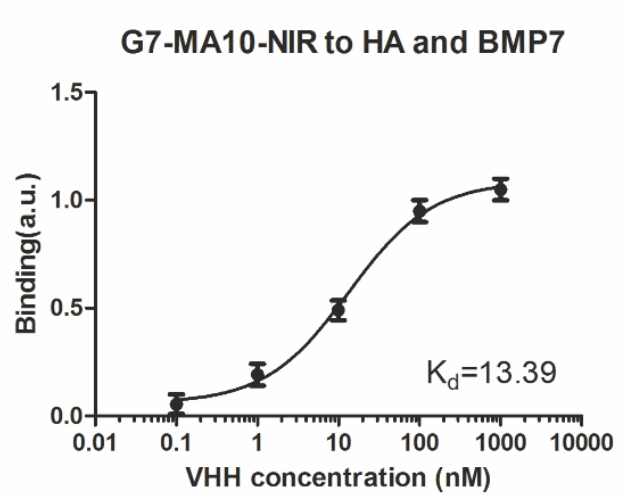




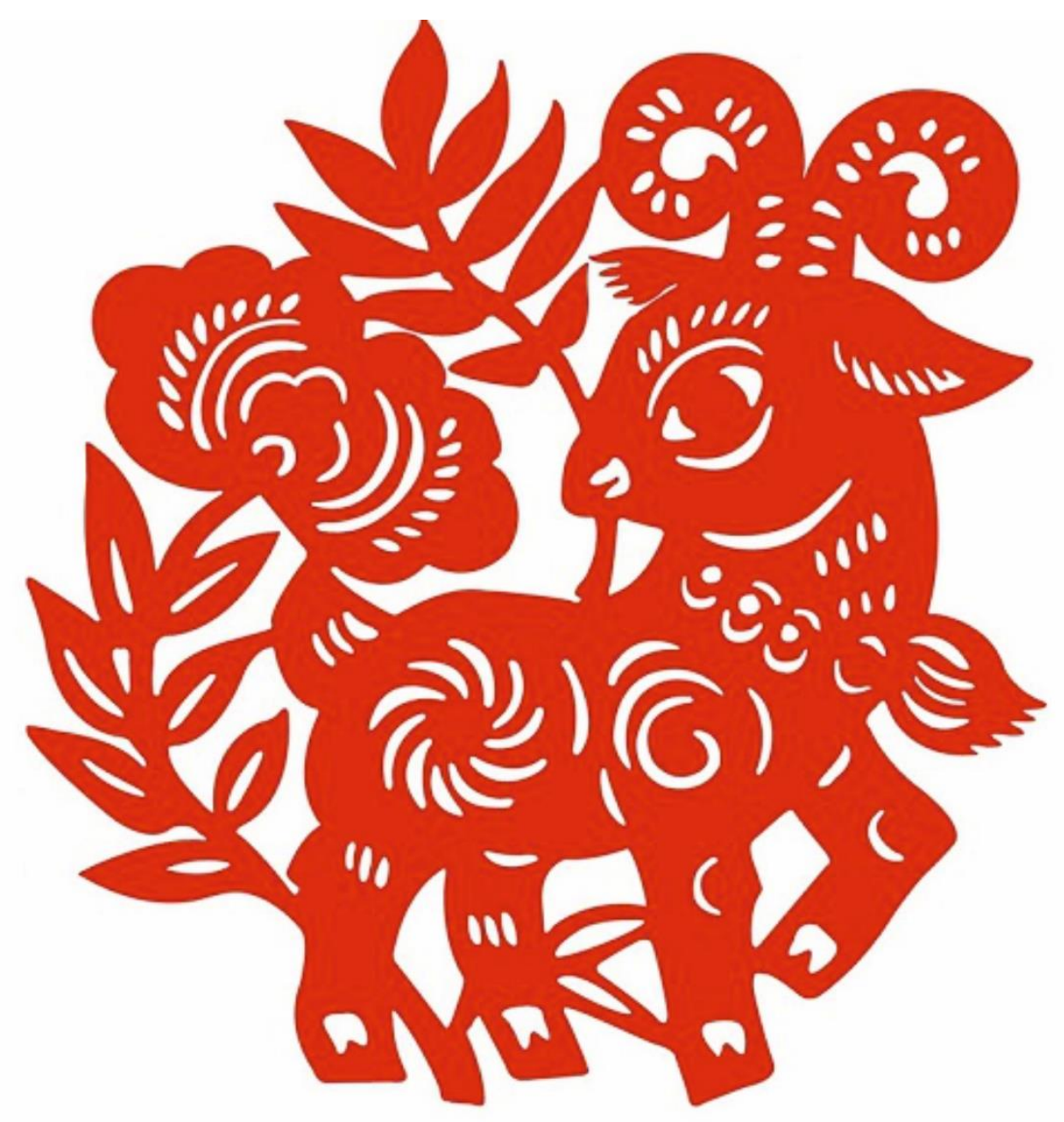




\section{Chapter 8}

\section{General Discussion and Outlook}

This chapter is an overall discussion of the entire thesis, with future perspectives.

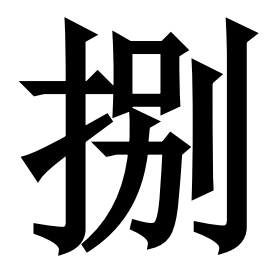

道生一，一生二，二生三，三生万物。

Tao gave birth to the One. The One gave birth successively to two things, three things, up to ten thousand. 


\section{The complexity of Osteoarthritis}

Osteoarthritis (OA) is a multifactorial disease that affects the whole joint. Patients of OA suffer serious pain and inconvenient movement. The initiation and progression of OA involve multiple risk factors, including mechanical injury, obesity, genetics and aging. Given the fact that $\mathrm{OA}$ is a complicated joint disease, much effort has been put into studying the mechanism underlying its pathogenesis. However, the exact mechanism is still poorly understood. There is still no effective strategy to stop its progression; patients with end-stage OA eventually undergo total joint replacement surgery [1]. The degeneration of articular cartilage has been thought to be the primary change during OA, and a combination of cellular changes and biomechanical stresses causes secondary changes, such as subchondral bone remodeling, the formation of osteophytes and bone marrow lesions [2-5]. Understanding molecular changes in the development of OA is important, especially in the early stage, since these changes could still be reversible, and treatment could slow or reverse further progression of the disease [6].

Biomarker measurement in bodily fluid such as serum, plasma and synovial fluid can be used to assess the joint's health status, the disease progression or the response to therapy, which is important for providing early treatment/intervention for OA. In this thesis, we therefore first measured the WNT signaling antagonists DKK1 and FRZB and the BMP antagonist GREM1 in synovial fluid and serum samples from a cross-sectional cohort of end stage OA patients eligible for total joint replacement and analyzed the correlation between these signaling factors and various structural and inflammatory markers of OA. The cohort consisted of 4 arms. Part of the patients received treatment with the Non-Steroidal Anti-Inflammatory drugs (NSAIDs) Naproxin and Celecoxib in the weeks prior to surgery (Chapter 3). This design allowed us to evaluate the effect of NSAIDs on the expression of DKK1, FRZB and GREM1. We had previously identified DKK1, FRZB and GREM1 as critical regulators of cartilage homeostasis by preventing hypertrophic differentiation of articular chondrocytes. In articular cartilage, their mRNA expression inversely correlated with the severity of osteoarthritis [7]. In line with this previous study, we found that DKK1 and FRZB in synovial fluid or serum also negatively correlated with OA severity. Celecoxib is a nonsteroidal anti-inflammatory drug used to treat 
pain and inflammation. We found that the synovial fluid FRZB level was increased after patients received celecoxib treatment. Considering the role of FRZB in joint homeostasis and the positive effects of celecoxib on human OA cartilage $[8,9]$, the beneficial effects of celecoxib treatment in patients by inducing the expression of an potentially anabolic factor like FRZB, by counteracting catabolic WNT-signaling, is of considerable interest. Our data further provides information about the clinical use of celecoxib in the treatment of OA. Inflammatory cytokines such as interleukin1 (IL1) and tumor necrosis factor $\alpha(\mathrm{TNF} \alpha)$ in the synovial fluid (SF) usually contribute to the degeneration of articular cartilage in OA patients, by triggering the upregulation of catabolic gene expression [10-12]. We found that the expression of DKK1 and FRZB in synovial fluid was negatively correlated with inflammatory factors such as IL1 $\beta$, NO and PEG2, while the expression of GREM1 positively correlated with these factors. The low level of DKK1 and FRZB in synovial fluid from OA patients may be caused by the high level of inflammatory factors. OA has long been considered a non-inflammatory disease. Our study provides more evidence on inflammation as part of the pathogenesis of OA. DKK1, FRZB and GREM1 could be used as potential biomarkers for diagnosing OA and for the assessment of the effects of medicines in clinic. The effectiveness and accuracy of these factors as biomarkers need to be further verified in future research.

\section{Cell-based OA therapy}

The most common cell sources for cartilage tissue engineering include articular chondrocytes and mesenchymal stem cells. Autologous chondrocyte implantation (ACI), which was introduced by Brittberg et al in 1994 [13], is the only clinically approved cell-based strategy that has been commonly used to repair articular cartilage defects with promising results. This approach is based on the use of chondrocytes from the non-weight-bearing relatively healthy area of articular cartilage, followed by amplifying in monolayer culture for approximately 6 weeks and reimplantation at the lesion site. However, monolayer expansion leads to a rapid chondrocyte dedifferentiation characterized by loss of the typical chondrocyte phenotype, so that fibrous cartilage rather than hyaline cartilage will form after implantation [14-17]. In 
addition, during OA progression, chondrocytes in vivo will also dedifferentiate with loss of normal phenotype, reduced production of matrix-related proteins, and finally advocating cartilage degeneration. Therefore, how to successfully redifferentiate the dedifferentiated chondrocytes is extremely important in cell-based therapies for cartilage repair.

Cartilage is an avascular tissue, and therefore chondrocytes are metabolically adapted to a lowoxygen tension, which is estimated to range from $7 \%$ on the joint surface to $1 \%$ close to subchondral bone [18]. Chondrocytes that are cultured in $21 \%$ oxygen usually change their normal phenotype and get a fibroblastic morphology [19], while hypoxic condition can play a crucial role in chondrocyte biology and restore the chondrogenic ability of dedifferentiated chondrocytes [20, 21]. In addition, accumulating evidence has shown that inflammation is involved in the pathogenesis of OA, and the expression of inflammatory factors such as IL1 $\beta$ and TNF $\alpha$ is observed in OA synovial fluid. In our study, we used hypoxia incubator with a setting of $2.5 \% \mathrm{O}_{2}$ to better mimic the physiological conditions of cartilage tissue. We studied the responses of healthy and OA chondrocytes to hypoxia and inflammatory cytokines in 3-D redifferentiating chondrocyte pellets (Chapter 5). We found that a high oxygen level of 21\% $\mathrm{O}_{2}$ concentration inhibited chondrocyte redifferentiation, particularly of OA chondrocytes, derived from macroscopically degenerated cartilage, while a low oxygen level of $2.5 \% \mathrm{O}_{2}$ concentration promoted chondrocyte redifferentiation and diminished the difference between relatively healthy and OA chondrocytes derived from the same joint. Our study suggests that redifferentiation of chondrocytes should be performed in 3-D cultures under hypoxic condition. The optimal level or timing of hypoxia for cultures still needs to be determined in future studies. In addition, we also found that hypoxia sensitized the chondrocyte's response to IL1 $\beta$ treatment in comparison to normoxia. Comparison of the basal expression levels of IL1 $\beta$ target genes in normoxia and hypoxia consistently showed that the expression in normoxia is higher. This may imply that culturing chondrocytes in normoxia itself may be sufficient to induce a mild inflammatory stress response. The combined effects of these two conditions may further deteriorate the chondrocyte's function, which would consequently contribute to OA progression. In this study, different expression profiles of HIF $1 \alpha$ and HIF $2 \alpha$ upon oxygen tension and IL1 $\beta$ stimulation were also observed in relatively healthy and OA chondrocytes. 
This indicates that a switch between HIF $1 \alpha$ and HIF $2 \alpha$ may be involved in the pathogenesis of OA. Based on this observation, we further looked at the synergistic effects of exogenous BMP7 and TGF- $\beta 3$ on the redifferentiation of OA chondrocytes, which cultured in a hypoxia incubator (Chapter 6). We found that the combination of BMP7 and TGF- $\beta 3$ can successfully direct OA chondrocytes to healthy chondrocyte phenotype, as was evidenced by the enhancement of the expression of cartilage markers and the promotion of cartilage formation under hypoxia. The combined beneficial effects of BMP7 and TGF- $\beta 3$ on OA chondrocytes indicate that healthy chondrocytes can be replaced by OA chondrocytes in the presence of $\mathrm{BMP7}$ and TGF- $\beta 3$ in an ACI procedure.

Although ACI shows promising results for the treatment of OA, the harvest of cells from cartilage leads to donor site morbidity and monolayer-expanded cells lose their phenotype rapidly. Replacement of chondrocytes by alternative cell sources like stem cells can potentially avoid these problems. MSCs are multipotent cells which can be derived from the bone marrow. They can be expanded in vitro and differentiated into the osteogenic, chondrogenic, and adipogenic lineages [22], and are considered suitable candidates for the promotion of tissue regeneration $[23,24]$. In addition, they can be easily isolated and massively expanded in culture. In both animal models and clinical studies, implanted MSCs were integrated into the defect site, which delayed the progression of OA $[25,26]$. MSCs are capable of regulating the immune response and adjusting the internal microenvironment in joints. They can also initiate endogenous repair mechanisms in the OA joint through direct cell-cell interaction or the secretion of various factors [27]. The medical work is beginning to consider using MSCs instead of chondrocytes to restore or regenerate the function of articular cartilage in OA. However, hypertrophic differentiation and subsequent endochondral ossification occur after implantation [28-30]. A study about the mechanism underlying hypertrophic differentiation would be of particular interest for the application of MSCs in the orthopedic clinic. Since Wnt signaling is indicated to be involved in the chondrogenic differentiation of MSCs, Chapter 4 recapitulates the dual role of WNT signaling in the chondrogenic differentiation of human mesenchymal stem cells (hMSCs). We observed that a low level of WNT signaling increased cartilage marker gene expression, elevated matrix deposition and inhibited the chondrocytes 
hypotrophy. A high level of WNT signaling showed opposite effects, which indicates that regulating the critical level of WNT signaling is important for the chondrogenesis of hMSCs.

BMPs are multi-functional cytokines that belong to the TGF- $\beta$ superfamily. BMP7, one of the BMPs, plays a key role in both cartilage and bone biology. Functionalization of biomaterial surfaces with BMP7 is a promising strategy to stimulate tissue repair and potentially tissue regeneration. However, chemical modification of BMP7 could lead to the loss of biofunction, which limits its application in tissue engineering. Chapter 7 deals with the immobilization of BMP7 on bone surfaces while preserving its bioactivity. We describe a non-covalent strategy for binding BMP7 to bone surface by using engineered bispecific antibodies (highly specific single domain antibody fragments of heavy chain only antibodies of Camelidae (VHH)), which bind to both hydroxyapatite (HA) and BMP7. After making this bispecific VHH, its biological activity was tested in vitro and in vivo. The result shows that bispecific VHH successfully directed BMP7 to bone and exerted its downstream signal transduction. Our study demonstrates that engineering bispecific VHH could be a versatile strategy for the functionalization of biomaterials with growth factors without affecting its bioactivity. In the future, this platform can be fine-tuned to target different growth factors such as DKK1, FRZB and TGF $\beta$, which are relevant for cartilage tissue engineering. Application of this technology in the ACI procedure could potentially induce cartilage formation. 


\section{References}

1. van der Kraan, P.M. and W.B. van den Berg, Chondrocyte hypertrophy and osteoarthritis: role in initiation and progression of cartilage degeneration? Osteoarthritis Cartilage, 2012. 20(3): p. 223-32.

2. Goldring, M.B. and S.R. Goldring, Articular cartilage and subchondral bone in the pathogenesis of osteoarthritis. Ann N Y Acad Sci, 2010. 1192: p. 230-7.

3. Pollard, T.C., S.E. Gwilym, and A.J. Carr, The assessment of early osteoarthritis. J Bone Joint Surg Br, 2008. 90(4): p. 411-21.

4. Dequeker, J. and F.P. Luyten, The history of osteoarthritis-osteoarthrosis. Ann Rheum Dis, 2008. 67(1): p. 5-10.

5. Ding, C., et al., What can we learn about osteoarthritis by studying a healthy person against a person with early onset of disease? Curr Opin Rheumatol, 2010. 22(5): p. $520-7$.

6. Man, G.S. and G. Mologhianu, Osteoarthritis pathogenesis - a complex process that involves the entire joint. J Med Life, 2014. 7(1): p. 37-41.

7. Leijten, J.C., et al., Gremlin 1, frizzled-related protein, and Dkk-1 are key regulators of human articular cartilage homeostasis. Arthritis Rheum, 2012. 64(10): p. 3302-12.

8. El Hajjaji, H., et al., Celecoxib has a positive effect on the overall metabolism of hyaluronan and proteoglycans in human osteoarthritic cartilage. J Rheumatol, 2003. 30(11): p. 2444-51.

9. de Boer, T.N., et al., The chondroprotective effect of selective COX-2 inhibition in osteoarthritis: ex vivo evaluation of human cartilage tissue after in vivo treatment. Osteoarthritis Cartilage, 2009. 17(4): p. 482-8.

10. Kobayashi, M., et al., Role of interleukin-1 and tumor necrosis factor alpha in matrix degradation of human osteoarthritic cartilage. Arthritis Rheum, 2005. 52(1): p. 12835.

11. Wojdasiewicz, P., L.A. Poniatowski, and D. Szukiewicz, The role of inflammatory and anti-inflammatory cytokines in the pathogenesis of osteoarthritis. Mediators Inflamm, 
2014. 2014: p. 561459.

12. Tetlow, L.C., D.J. Adlam, and D.E. Woolley, Matrix metalloproteinase and proinflammatory cytokine production by chondrocytes of human osteoarthritic cartilage: associations with degenerative changes. Arthritis Rheum, 2001. 44(3): p. 585-94.

13. Brittberg, M., et al., Treatment of deep cartilage defects in the knee with autologous chondrocyte transplantation. N Engl J Med, 1994. 331(14): p. 889-95.

14. Cournil-Henrionnet, C., et al., Phenotypic analysis of cell surface markers and gene expression of human mesenchymal stem cells and chondrocytes during monolayer expansion. Biorheology, 2008. 45(3-4): p. 513-26.

15. Hubka, K.M., et al., Enhancing chondrogenic phenotype for cartilage tissue engineering: monoculture and coculture of articular chondrocytes and mesenchymal stem cells. Tissue Eng Part B Rev, 2014. 20(6): p. 641-54.

16. Peterson, L., et al., Two- to 9-year outcome after autologous chondrocyte transplantation of the knee. Clin Orthop Relat Res, 2000(374): p. 212-34.

17. Roberts, S., et al., Autologous chondrocyte implantation for cartilage repair: monitoring its success by magnetic resonance imaging and histology. Arthritis Res Ther, 2003. 5(1): p. R60-73.

18. Fermor, B., et al., Oxygen, nitric oxide and articular cartilage. Eur Cell Mater, 2007. 13: p. 56-65; discussion 65.

19. von der Mark, K., et al., Relationship between cell shape and type of collagen synthesised as chondrocytes lose their cartilage phenotype in culture. Nature, 1977. 267(5611): p. 531-2.

20. Araldi, E. and E. Schipani, Hypoxia, HIFs and bone development. Bone, 2010. 47(2): p. 190-6.

21. Schrobback, K., et al., Effects of oxygen and culture system on in vitro propagation and redifferentiation of osteoarthritic human articular chondrocytes. Cell Tissue Res, 2012. 347(3): p. 649-63.

22. Pittenger, M.F., et al., Multilineage potential of adult human mesenchymal stem cells. Science, 1999. 284(5411): p. 143-7. 
23. Worster, A.A., et al., Chondrocytic differentiation of mesenchymal stem cells sequentially exposed to transforming growth factor-betal in monolayer and insulinlike growth factor-I in a three-dimensional matrix. J Orthop Res, 2001. 19(4): p. 73849.

24. Noth, U., A.F. Steinert, and R.S. Tuan, Technology insight: adult mesenchymal stem cells for osteoarthritis therapy. Nat Clin Pract Rheumatol, 2008. 4(7): p. 371-80.

25. Sato, M., et al., Direct transplantation of mesenchymal stem cells into the knee joints of Hartley strain guinea pigs with spontaneous osteoarthritis. Arthritis Res Ther, 2012. 14(1): p. R31.

26. Kasemkijwattana, C., et al., Autologous bone marrow mesenchymal stem cells implantation for cartilage defects: two cases report. J Med Assoc Thai, 2011. 94(3): p. $395-400$.

27. Qi, Y., G. Feng, and W. Yan, Mesenchymal stem cell-based treatment for cartilage defects in osteoarthritis. Mol Biol Rep, 2012. 39(5): p. 5683-9.

28. Freyria, A.M. and F. Mallein-Gerin, Chondrocytes or adult stem cells for cartilage repair: the indisputable role of growth factors. Injury, 2012. 43(3): p. 259-65.

29. Huey, D.J., J.C. Hu, and K.A. Athanasiou, Unlike bone, cartilage regeneration remains elusive. Science, 2012. 338(6109): p. 917-21.

30. Puetzer, J.L., J.N. Petitte, and E.G. Loboa, Comparative review of growth factors for induction of three-dimensional in vitro chondrogenesis in human mesenchymal stem cells isolated from bone marrow and adipose tissue. Tissue Eng Part B Rev, 2010. 16(4): p. $435-44$. 


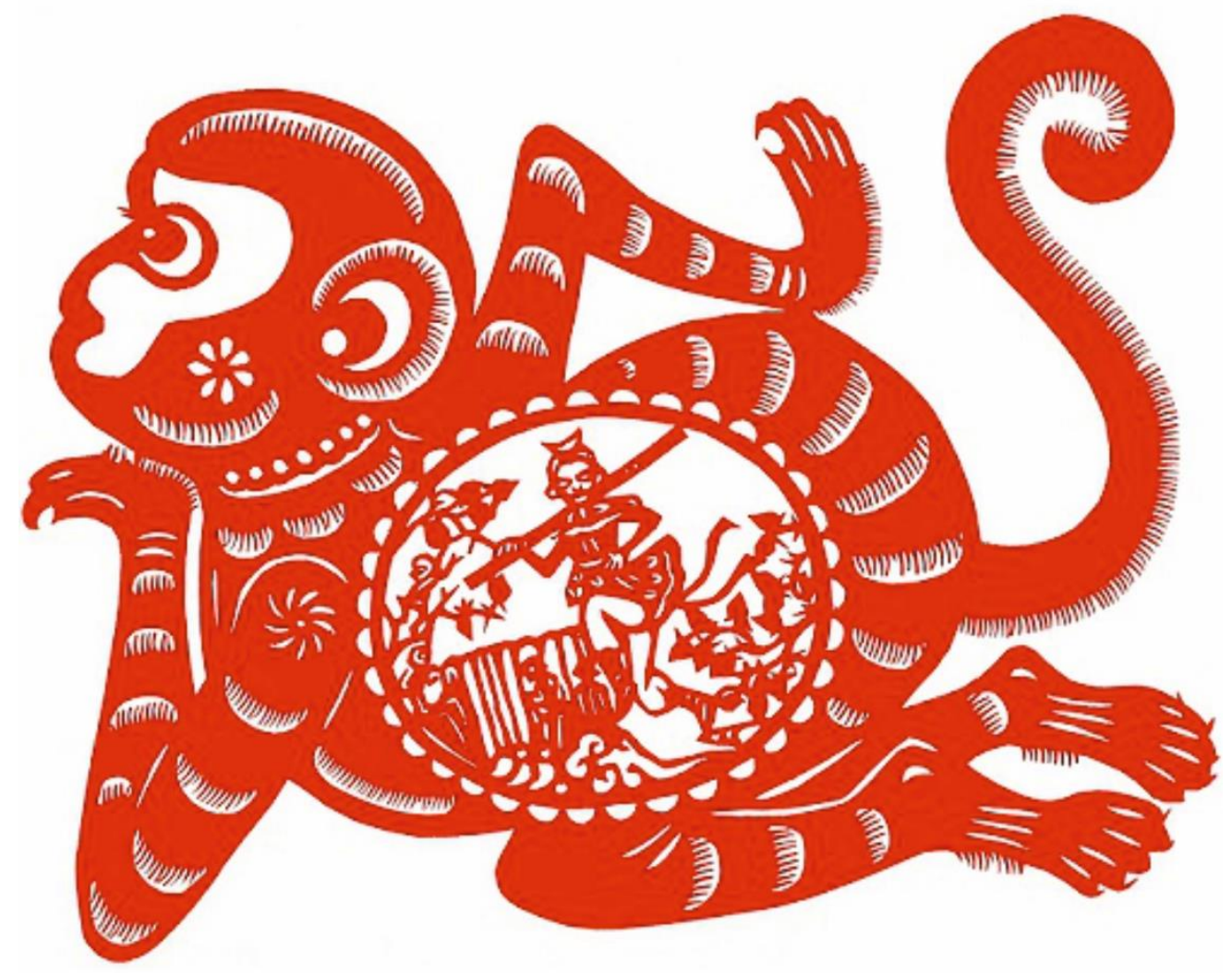




\section{Summary}

Cartilage is a connective tissue widely exist in the body. OA is one kind of universal chronic joint disease with progressive degradation of joint cartilage. Due to the avascular and aneural properties and limited repair capacity of cartilage, once it is irreparably damaged, external intervention in the form of cartilage repair is desired. Chondrocytes is the only one cell type in the articular cartilage to produce the matrix. Autologous chondrocyte implantation (ACI) and hMSC based cell therapy have been shown promising options. Multiple cytokines and signaling pathways have been shown to involved in the regulation of chondrocytes homeostasis and cartilage development. The aim of this thesis is to study the chondrogenic differentiation of human MSCs, redifferentiation of human chondrocytes and the possible BMP7 based tissue engineering by focus on Wnt signaling, hypoxia induced signaling, transforming growth factor$\beta$ (TGF- $\beta$ ) and bone morphogenetic protein (BMP) signaling, to provide more mechanisms in the ACI and hMSCs based cell therapy for OA.

Hypertrophy occurs occasionally in osteoarthritis (OA). In order to have an clear insight into the exact regulation of hypertrophy by the signaling network for the efficient application of MSCs or chondrocytes in the OA therapy, we provided a comprehensive review on recent literatures describing signal pathways in the hypertrophy of MSCs derived chondrocytes and chondrocytes, in chapter 2.

From chapter 2, we know that Wnt signaling is extremely important in the regulation of chondrocyte biology. Therefore, in chapter 3, we investigated the presence of WNT antagonists Dickkopf-related protein 1 (DKK1), Frizzled-related protein (FRZB) and Gremlin 1 (GREM1) in synovial fluid (SF) and serum respectively from osteoarthritis (OA) patients and correlate these antagonists with pro-inflammatory cytokines in SF. In this study, a comprehensive cross sectional analysis was performed in synovial fluid and serum samples collected from OA patients, through measuring the concentration of DKK1, FRZB, GREM1 and inflammatory factors in synovial fluid and serum in patients by ELISA. The results demonstrates that the important involvement of DKK1, FRZB and GREM1 in OA and their expression was 
associated with inflammation factors, suggesting DKK1 and FRZB can be potential biomarkers for joint disease.

Since Wnt signaling is important in the chondrocytes differentiation, we further elucidated the effects of Wnt activator and Wnt inhibitor on the chondrogenic differentiation of hMSCs by continuous inhibiting or activation Wnt signaling in chapter 4 . We found the addition of Wnt activator BIO downregulated the expression of cartilage specific genes and decreased cartilage matrix while Wnt inhibitor PKF118-310 showed upregulated the expression of cartilage markers and increased cartilage matrix deposition. Our findings suggest that fine tune Wnt signal in the chondrogenic differentiation process of hMSC could contribute to the cartilage tissue engineering.

Chondrocytes usually go to dedifferentiation after expansion in monolayer in ACI, so the redifferentiation is required. In chapter 5 , we investigated the effects of oxygen on threedimensional redifferentiation in both healthy and OA chondrocyte with or without IL1 $\beta$ stimulation. We found hypoxia dramatically promoted the redifferentiation of OA chondrocytes and diminished the deference between HL and OA chondrocyte. However, chondrocytes become more sensitive to IL1 $\beta$ treatment in hypoxic conditions. This data provide information about how the chondrocyte adapt to a low oxygen tension and inflammatory environment through the modulation of anabolic genes and catabolic genes and survival genes HIF $1 \alpha$ and HIF2 $\alpha$.

In chapter 6, we further optimize the redifferentiation conditions of chondrocytes. We identified the effects of exogenous BMP7 and TGF- $\beta 3$ on the redifferentiation of OA chondrocytes under hypoxia. In this research, we found either TGF- $\beta 3$ or BMP7 only was not enough to direct the fully redifferentiation of OA chondrocytes, while the combination of TGF$\beta 3$ and BMP7 synergistically induced OA chondrocytes development into health chondrocytes both in normoxia and hypoxia. This study indicated that the fine combination of cytokines or growth factor and the balance between signaling pathways is a requirement for normal chondrocyte differentiation and cartilage development. 
Growth factors are usually used in the tissue engineering. In chapter 7, we generated biofunctional bihead VHH (specific single domain antibody fragments of heavy chain antibodies of Camelidae) that can specific bind BMP-7 and HA simultaneously. In this study, BMP7 was efficiently direct to the mineralized skeleton through binding bihead after injection into mice. This study demonstrate the bihead VHH is a promising strategy for biofunctionalization of scaffolds in tissue engineering without modification of growth factor. In future, the same strategy can be used in cartilage repairing.

All together, this thesis evaluated the Wnt signaling in synovial fluid and the differentiation of hMSCs, assessed the redifferentiation of human chondrocytes effected by hypoxia, inflammatory factor IL1 $\beta$, BMP7 and TGF $\beta 3$, elucidated the production of bihead VHHs and its application in tissue engineering. We believe the findings from this thesis may offer great potential in cell-based cartilage engineering. 


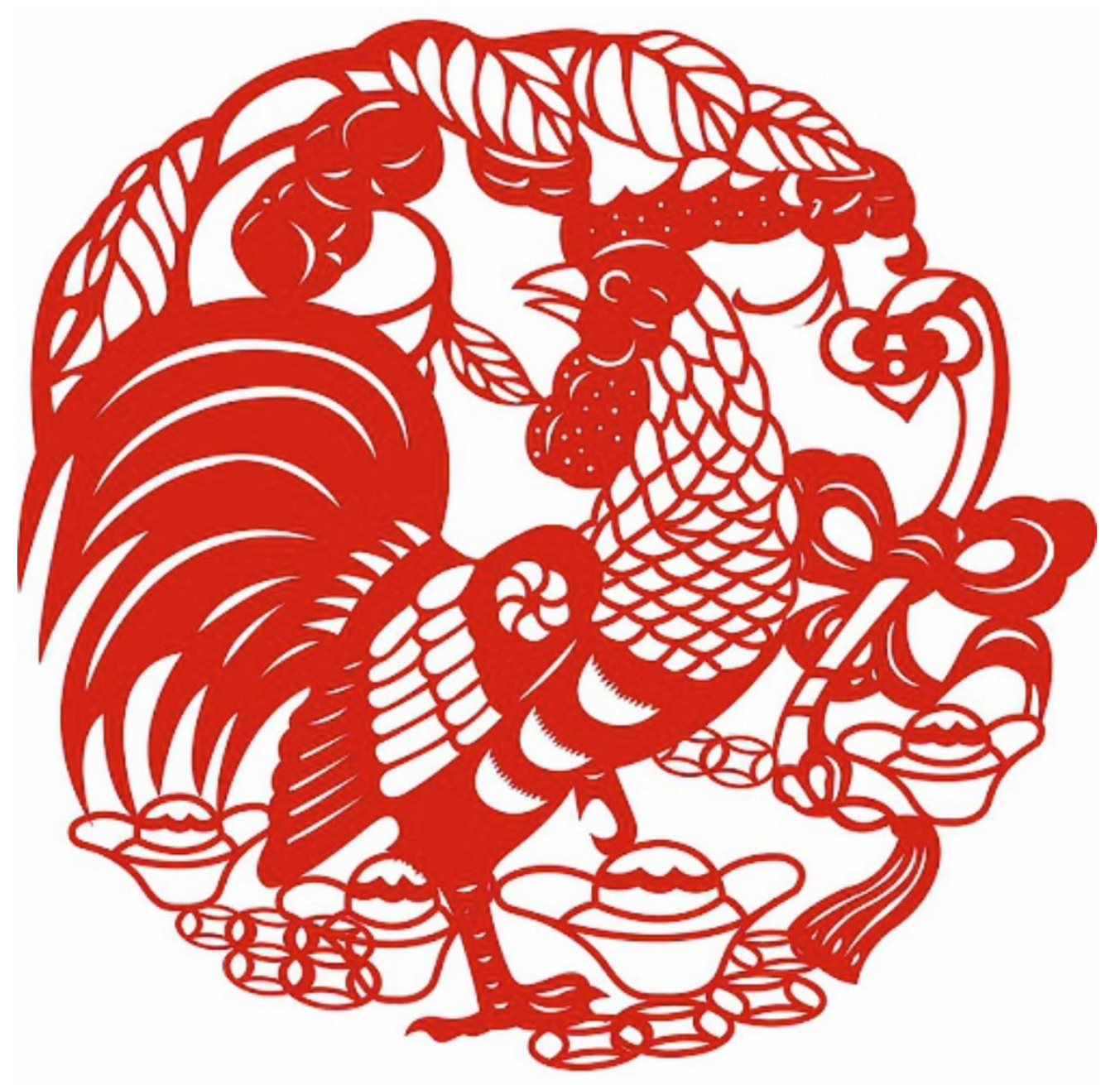




\section{Acknowledgements}

Finally my thesis are going to this part. Many things and scenes fly into my mind when I think back what I have done and what life I have lived during the past years. All laborious work is worth. I feel whole body is strength right now. However, without the encouragements and supports from you lovely people, I can not successfully finish it. Therefore, it is the right time to thank all you guys to help me during the past years.

First of all, I would like to thank my supervisor, Prof. Marcel Karperien, for giving me the opportunity to finish my this $\mathrm{PhD}$ research in Develpmental BioEngineering group. Your insightful and deep thoughts always kindled the light of my mind. There are seem never exist problem in your smart brain. Thank you for guiding me to the sea of research in a right way. I believe that I will go farther and farther with the light that you ignited for me, even when I am in a new life and a new circle in future.

I want to thank particularly Dr. Janine Post for giving the chance to Leilei to pursue her $\mathrm{PhD}$ in Twente and training her a very good wife. Thank you for the deep disscussion and winged suggestion both in our research and life. I have never forget that your optimistic attitude on the research and your perfect Chinese pronunciation. You contribute too much to our happy family!

I want to thank as well Prof. Dr. R. Passier, Prof. Dr. M. Claessens, Dr. J. Prakash, Prof. Dr. Peter van der Kraan, and Dr. T. Welting for being part of my graduation committee. I am very appreciate you taking time to discuss my work.

To my paranymphs: Honglin (洪林) and Xiaokui (小奎). Thank you for being together with me at this important moment. Honglin, you are the lucky man to marry my friend Minmin (敏 敏) who is diligent, kind, competent and virtuous women. Xiaokui, thank you and your manager, Xiong (熊总), you always tried to take us out to enjoy the nice weather and food. You have put so much love to Meilan. Meilan will remember his “dried father” (干爹) and “dried mother” (干妈) forever. 
Then, many thanks the pepole in DBE group for all the support. Piet, I am very appreciate that you always treat my wife Leilei and my friend Rong well. Jacqueline, you are always ready to help Leilei and me. Thanks for the toys that you bring it to my older son, Meilan. Sanne, thank you for teaching me to do the MMA embeding experiments. Jan, thank you for being activist in our DBE group and the suggestions in my research and thesis. Tom, thank you for the warm words when I am doing the experiments. Elahei, you always say to us in Persian language, which is very good for the break. Kannan, thank you for the delicious Indian food and nice topics. Yao (尧), I am very gald that we can talk in chinese always. Corina, I really like your open personality. Milou and Ingrid, I am very grateful to both of you for babyshower, it was a big surprise. Brenda, you are really an actor with great promise, Leilei that you acted is better than the real one. Maurice, Joao, Sieger, Jeroen, Lisanne, Bram, Janneke and other DBE members, thank you sharing ideas and discussions in the labs and DBE group meetings.

The following thanks are given to my chinese friends. It's my great pleasure to meet so many lovely friends in Enschede. Rong (荣), you are the first person my wife met in Netherlands. Thanks for sharing happiness and frustration with us. We are very happy to see that you will have happy life with Xiaolin (小林). You love Meilan so much and always take sometime to visit us to see him. Meilan already start to go to school and could chat with his lovely Gugu (姑姑). Minmin (敏敏), the Shenshen (婶婶) of my son, thanks for living with us for a long time, you are the right person to witness the grow-up process of Meilan from Zero. You will have very happy life with Honglin. I hope you can give birth to a lot babies for Honglin. Xiaohua (小华), Kenan (柯楠), it's great to have both of you as friends, thanks for giving the rid when we left The Netherland. Jiahui, Liuyao, thanks for sending my wife to hospital during delivery and pick us up after two hours. we shared the happiness of first-time parent and the joy of raising the little one. Jingwei (静微), Zhonghua (中华), you are always hospitality. Niehui (聂辉), Penghuan (彭欢), its nice to meet both of you here. Wenlong (文龙), Duying (杜颖), Lantian (蓝天), Xingwu (醒悟), Zhuola (卓拉), Wanglei (王否), Lulu (露露), Yali (雅 丽), Hainan (海楠), Hairong (海荣), Wangyi (王毅), Aijie (爱洁), Zeng (曾哥), Rong (大荣 姐) and other chinese friends, thank you all for being around, making my this PhD life cheerful and colorful. I wish you all the best! 
Finally, special thanks to my family. My Mom and Dad, my brother, my wife, my older son (黄 美兰沁) and my younger son (黄美承康), thank God that we are family. Hope we are happy and healthy forever.

Thanks to all the pepole who have been there to help and support me during the past years, I wish you all the best! Thanks!

15-12-2018

Xiaobin Huang

Philadelphia, USA 


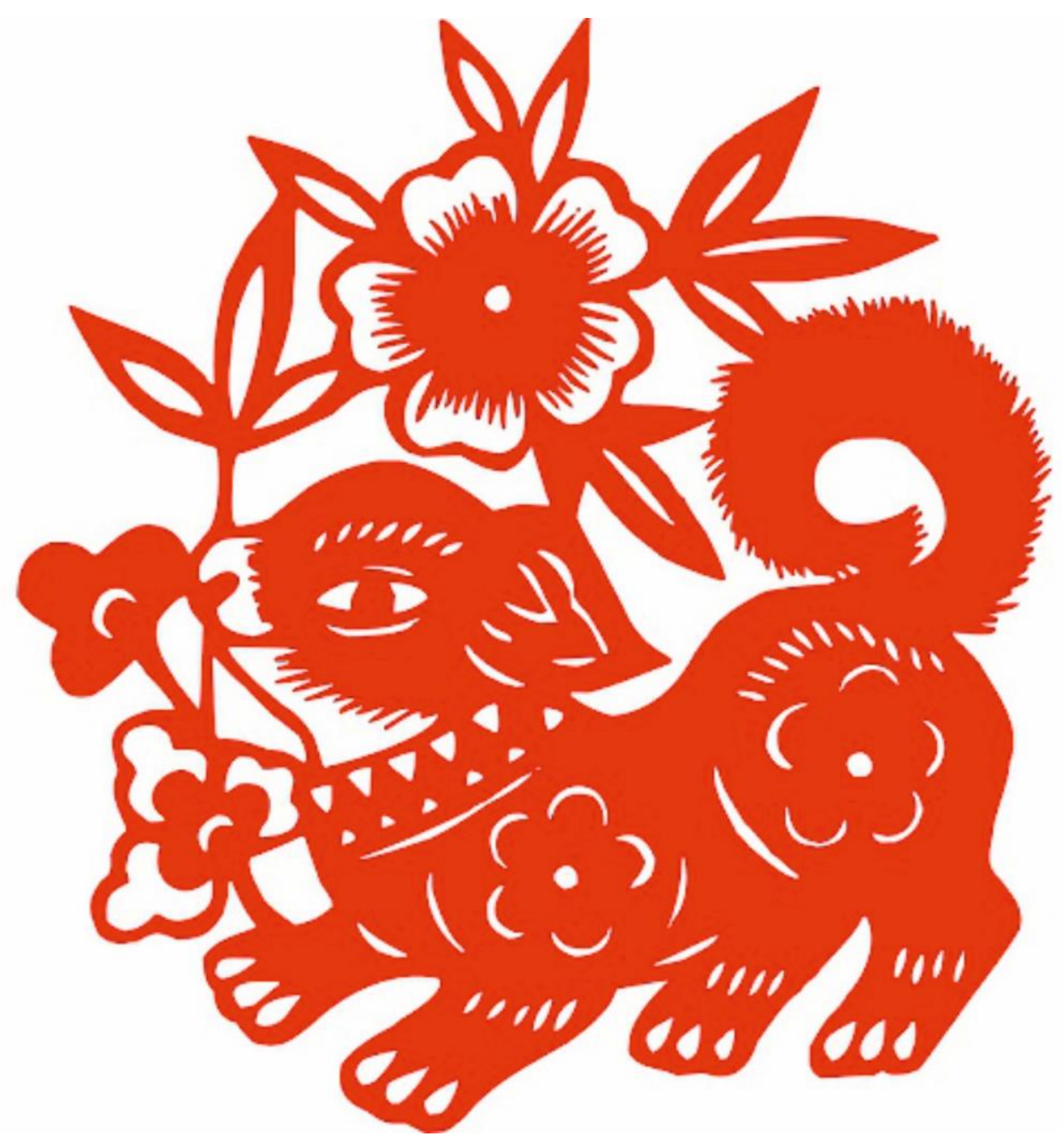




\section{Curriculum Vitae}

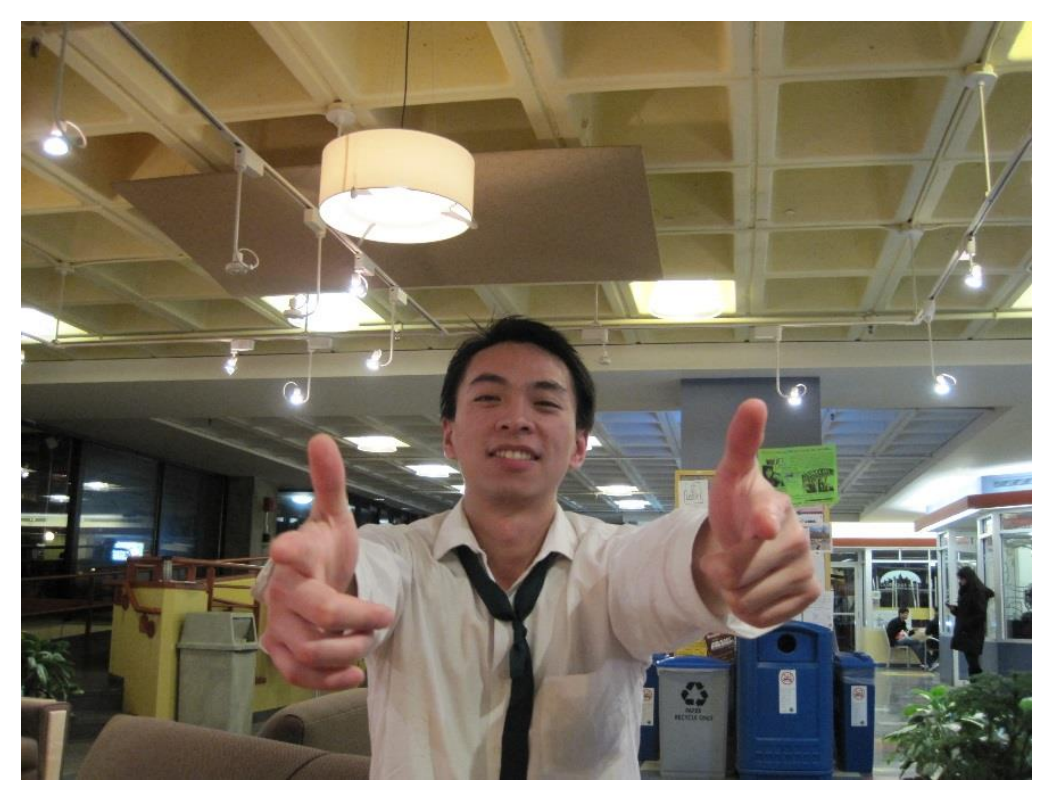

Xiaobin Huang was born on $1^{\text {st }}$ of September 1980 in Chongqing, China. In 2002, he received his Bachelor's degree in biology from Jilin Argricultural University, Changchun, China. In 2005, he started his master program in radiation medicine at Peking Union Medical College,

Beijing, China. In 2008, as a visiting scholar, he studied the skeleton biology in Harvard dental school, the Forsyth Institute, Boston, US. In 2009, as a visting scholar as well, he studied the synthetic biology in Massachusetts Institute of Technology (MIT), Boston, US. In 2011 he worked as a lecturer in developmental biology in Chongqing University. In 2015, he started his $\mathrm{PhD}$ project in Developmental BioEngineering group in University of Twente under supervision of Prof. Marcel Karperien. In this peorid, he mainly focus on the role of WNT, WNT antagonists, BMP7, TGF- $\beta 3$ and IL-1 $\beta$ in cartilage development and degeneration. In 2018, he completed his $\mathrm{PhD}$ thesis entitled "Multi-signals involved in human joint hemeostasis". 


\section{List of publications}

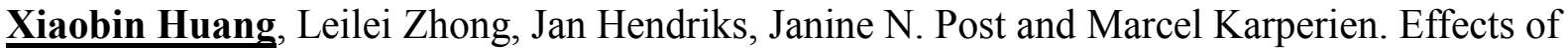
small molecules BIO and PKF118-310 on chondrogenic differentiation of human mesenchymal stem cells. Submitted.

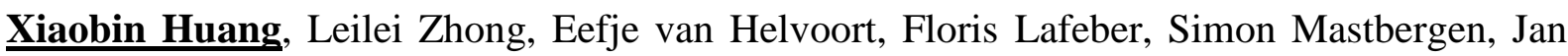
Hendriks, Janine N. Post, Marcel Karperien. The expression of DKK1 and FRZB are negatively correlated to local inflammation and OA severity. Submitted.

Xiaobin Huang, Leilei Zhong, Jan Hendriks Janine N. Post, Marcel Karperien. Hypoxia greatly promotes the redifferentiation of human osteoarthritic chondrocytes but sensitize it to IL-1 $\beta$ stimulation. Submitted.

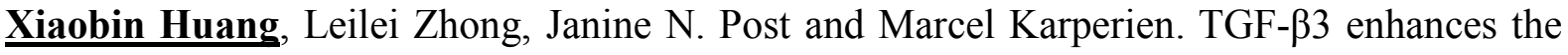
osteoarthritis chondrocytes response to BMP-7 during redifferentiation under hypoxia. Submitted.

Xiaobin Huang, Emilie Dooms Rodrigues, Jasper van Weerd, Leilei Zhong, Janine N. Post, Jeroen C.H. Leijten, Mohamed El Khattabi, Ivo Que, Theo Verrips and Marcel Karperien. Bihead VHHs efficiently direct BMP7 to bone in tissue engineering. In preparation.

Xiaobin Huang, Honglin Chen, Leilei Zhong, Marcel Karperien. Nano-electrospun is more benefit the chondrogenic differentiation of human MSC than micro-electrospun through downregulation canonical Wnt pathway. In preparation.

Huan Peng, Xiaobin Huang, Wenjing Xu, Andrea Götz, Marcel Karperien, Andrij Pich. Biodegradable Redox-Sensitive Nanogels Based on Water Soluble Reactive Cyclic NVinylamides Copolymers for Intracellular Drug Delivery. In preparation.(Co-first author)

Xiaobin Huang, Janine N. Post, Leilei Zhong, Staffan Larsson, Marcel Karperien, André Struglics. Dickkopf-related protein 1 and Gremlin 1 show different response than Frizzledrelated protein in human synovial fluid following knee injury and in patients with osteoarthritis. Submitted to osteoarthritis and cartilage.

Xiaobin Huang, Yong Hou, LeiLei Zhong, Dechun Huang, Hongliang Qian, Marcel Karperien 
and Wei Chen. Promoted Chondrogenesis of Co-cultured Chondrocytes and Mesenchymal Stem Cells under Hypoxia Using In-situ Forming Degradable Hydrogel Scaffolds. Biomacromolecules. 2017 Dec 6. doi: 10.1021/acs.biomac.7b01271. [Epub ahead of print].

Leilei Zhong, Stefano Schivo, Xiaobin Huang, Jeroen Leijten, Marcel Karperien and Janine N. Post. Nitric Oxide Mediates Crosstalk between Interleukin $1 \beta$ and WNT Signaling in Primary Human Chondrocytes by Reducing DKK1 and FRZB Expression. Int J Mol Sci. 2017, 18, 2491; doi:10.3390/ijms18112491.

Honglin Chen, Xiaobin Huang, Minmin Zhang, Febriyani Damanik, Mattew B. Baker, Anne Leferink, Huipin Yuan, Roman Truckenmü ller, Clemens van Blitterswijk, Lorenzo Moroni. Tailoring Surface Nanoroughness of Electrospun Scaffolds for Skeletal Tissue Engineering. Acta Biomater. 2017 Sep 1;59:82-93.

Wang B, Wang D, Zhao S, Huang X, Zhang J, Lv Y, Liu X, Lv G, Ma X. Evaluate the ability of PVP to inhibit crystallization of amorphous solid dispersions by density functional theory and experimental verify. Eur J Pharm Sci. 2017 Jan 1;96:45-52.

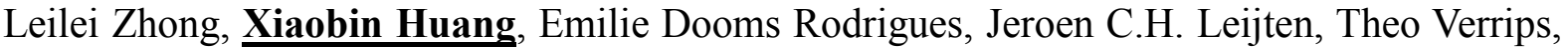
Mohamed El Khattabi, Marcel Karperien, Janine N. Post. Endogenous DKK1 and FRZB Regulate Chondrogenesis and Hypertrophy in Three-Dimensional Cultures of Human Chondrocytes and Human Mesenchymal Stem Cells. Stem Cells Dev. 2016 Dec 1;25(23):18081817. (Co-first author)

Huan Peng, Xiaobin Huang, Alex Oppermann, Andrea Melle, Lindsey Weger, Marcel Karperien, Dominik Wöll and Andrij Pich. A facile approach for thermal and reduction dualresponsive prodrug nanogels for intracellular doxorubicin delivery. Journal of Materials Chemistry B, 2016 Nov 4, 7572-7583. (Co-first author)

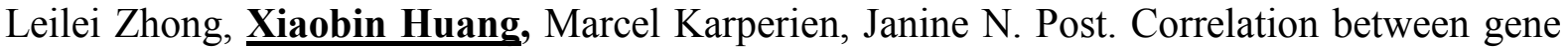
expression and osteoarthritis progression in human. Int J Mol Sci. 2016 Jul 14;17(7). (Co-first author)

Andrea Melle, Andreea Balaceanu, Michael Kather,Yaodong Wu, Elisabeth Gau, Wenjie Sun, Xiaobin Huang, Xiangyang Shi, Marcel Karperien and Andrij Pich. Stimuli-responsive poly(N-vinylcaprolactam-co-2-methoxyethyl acrylate) core - shell microgels: facile synthesis, 
modulation of surface properties and controlled internalisation into cells, Journal of Materials Chemistry B, $2016 \mathrm{Jul}$ 4, 5127-5137.

Le Yu, Xiaobin Huang, Wenfa Zhang, Huakan Zhao, Gang Wu, Fenglin Lv, Lei Shi, Yong Teng. Critical role of DEK and its regulation in tumorigenesis and metastasis of hepatocellular carcinoma. Oncotarget. 2016 May 3;7(18):26844-55. (Co-first author)

Huakan Zhao, Fenglin Lv, Guizhao Liang, Xiaobin Huang, Gang Wu, Wenfa Zhang, Le Yu, Lei Shi, Yong Teng. FGF19 promotes epithelial-mesenchymal transition in hepatocellular carcinoma cells by modulating the GSK $3 \beta / \beta$ - catenin signaling cascade via FGFR4 activation. Oncotarget. 2016 Mar 22;7(12):13575-86.

Huan Peng, Kristin Rübsam, Xiaobin Huang, Felix Jakob, Marcel Karperien, Ulrich Schwaneberg, and Andrij Pich. Reactive Copolymers Based on N-Vinyl Lactams with Pyridyl Disulfide Side Groups via RAFT Polymerization and Postmodification via Thiol-Disulfide Exchange Reaction. Macromolecules, 2016, 49 (19), pp 7141-7154

Leilei Zhong, Xiaobin Huang, Marcel Karperien, Janine N. Post. The Regulatory Role of Signaling Crosstalk in Hypertrophy of MSCs and Human Articular Chondrocytes. Int J Mol Sci. 2015 Aug 14;16(8):19225-47. (Co-first author)

Yang B, Tang Q, Post J, Zhou H, Huang XB, Zhang XD, Wang Q, Sun YM, Fan FY. Effect of radiation on the Notch signaling pathway in osteoblasts. Int J Mol Med. 2013 Mar;31(3):698706.

Xiaobin Huang, Leslie Morse, Yan Xu, Hana Sychrová, Phil Stashenko, Feiyue Fan, Ricardo Battaglino. Mutational Analysis of NHAoc/NHA2 in Saccharomyces cerevisiae. Biochimica et Biophysica Acta. 2010, 1800(12):1241-7.

Parayil Kumaran Ajikumar, Wen-Hai Xiao, Keith E. J. Tyo, Yong Wang, Fritz Simeon, Effendi Leonard, Oliver Mucha, Too Heng Phon, Blaine Pfeifer, Gregory Stephanopoulos. Isoprenoid Pathway Optimization for Taxol Precursor Overproduction in Escherichia coli. Science, 2010. 330(6000): p. 70-4. We thank Xiaobin Huang for helping with experiments and suggestions. 


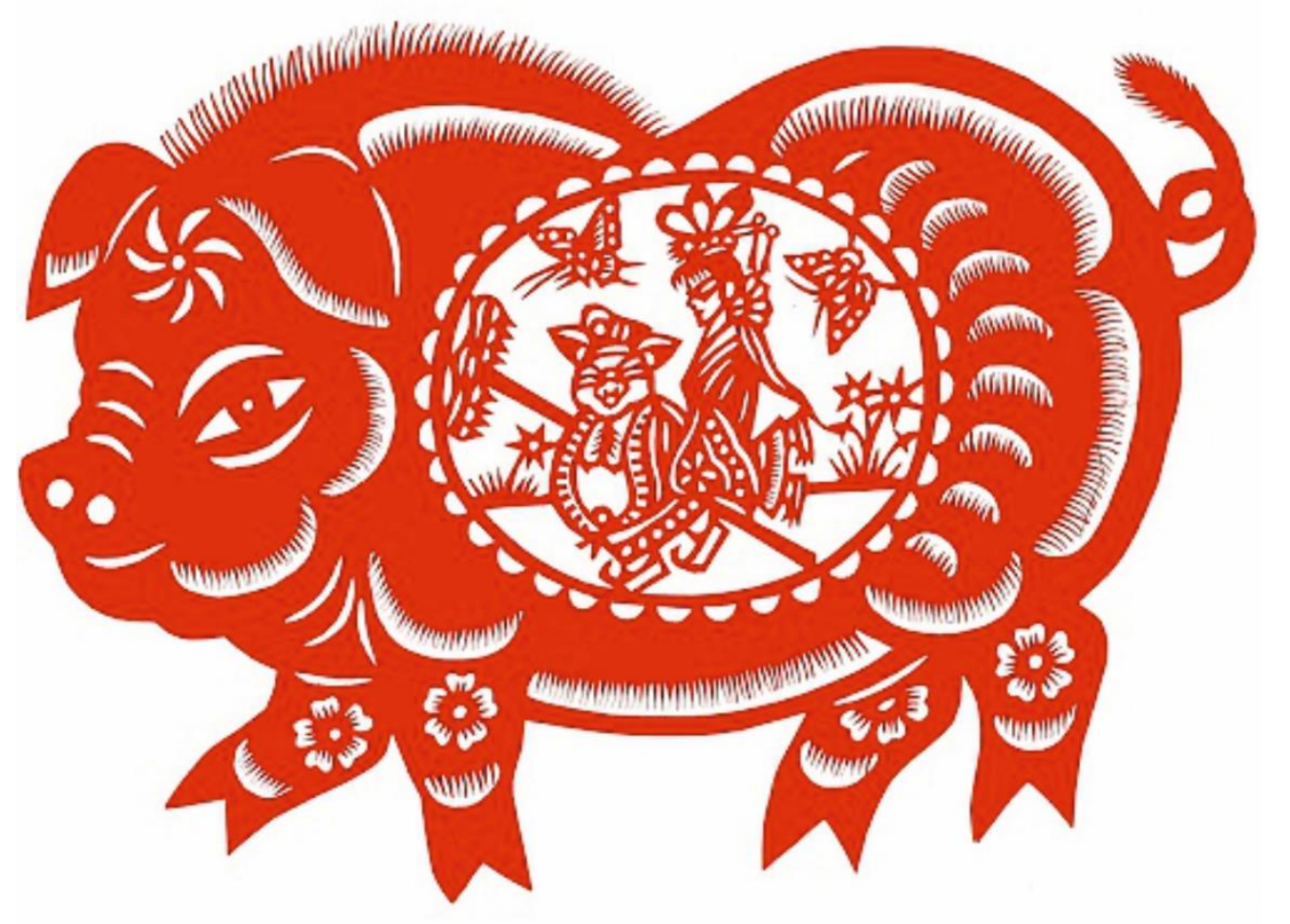

